\title{
MODERN SPECTRAL ANALYSIS IN HF RADAR REMOTE SENSING
}

\author{
Ascension VIZINHO
}

Thesis submitted for the degree of Doctor of Philosophy

The University of Sheffield School of Mathematics and Statistics

Applied Mathematics Section

April 1998

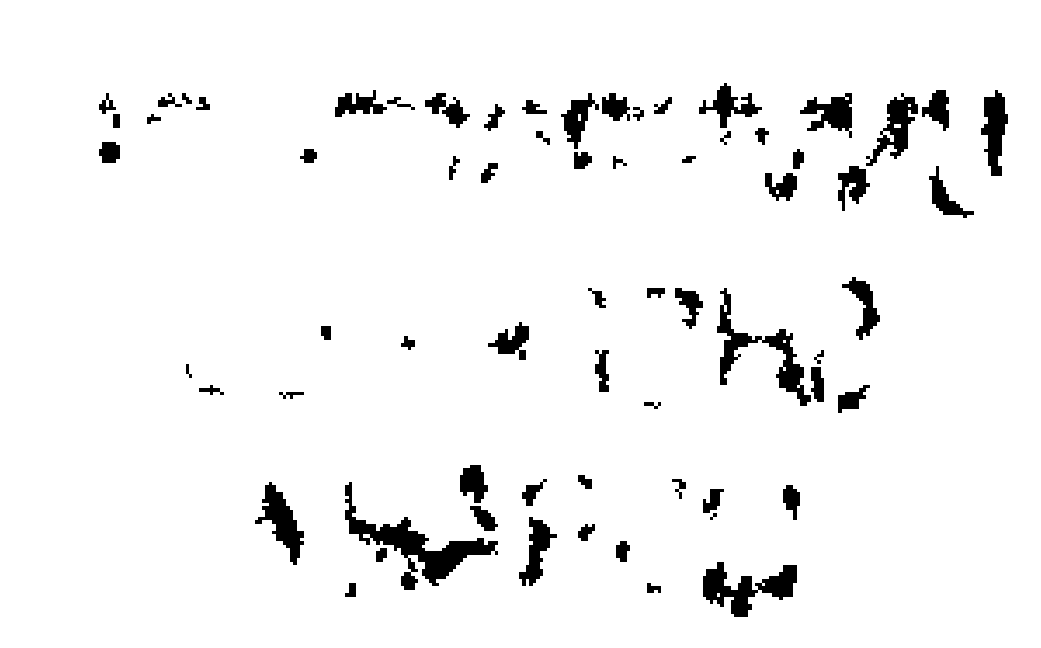




\title{
Modern Spectral Analysis in HF Radar Remote Sensing
}

\author{
A. Vizinho
}

\section{Summary}

High-Frequency (HF) radar systems are currently used to collect wave data. By applying spectral analysis methods, such as the Fast Fourier Transform (FFT) method, to the radar backscatter from the ocean surface, the so-called Doppler spectrum is calculated, and from this the directional wave spectrum and wave measurements are obtained.

Because of the random nature of the ocean surface, spectral measurements are subject to random variability. In order to reduce variability, and hence to obtain relatively precise estimates, each spectrum is usually calculated by averaging a number of FFT estimates. Naturally, this method requires long data series, and problems may arise. In rapidly varying sea conditions, for example, successive FFT estimates may be quite inconsistent with each other (in non-stationary conditions), and then the spectrum estimate obtained by averaging is not only difficult to interpret but it may also be distorted.

It is known that the more recent spectral analysis methods such as methods based on autoregressive (AR) and autoregressive-moving average (ARMA) stochastic models can provide stable estimates from short data sets. Thus these methods are potentially good alternatives to the FFT, as they avoid problems inherent to the use of large data sets. The aim of this thesis is to investigate how some of the modern spectral analysis methods may be used to obtain reliable spectral estimates from small data sets.

Unlike the FFT method, the AR- and ARMA-based methods presuppose specific parametric forms for the spectral function, and therefore consist in estimating certain parameters from the data (as opposed to estimating the function itself). The modified covariance method and Burg's method are among several methods of estimating the parameters of the spectral function. 
The choice between the different models is based on the shape of the Doppler spectra; it is concluded that an $\mathrm{AR}$ process conforms better with the available data. Among the various estimation methods, the modified covariance method proves to be more reliable. In order to validate our choice, we carried out several comparisons with the FFT method. The modified covariance method with smaller data sets is shown to give satisfactory results, comparable to those of the FFT method as usually applied, while the FFT method with smaller data sets performs poorly. Accurate wave measurements are still obtained by reducing the amount of data by a factor of four.

The calculation of directional wave spectra from Doppler spectra estimated with the new technique also gave satisfactory results. 


\section{ACKNOWLEDGEMENTS}

This work could not have been carried out without the help and guidance of a number of people all of whom I am grateful to.

First of all, I want to thank my supervisor Dr. Lucy Wyatt for her support and patience during this work. I also wish to thank Dr. Clive Anderson for his help on statistical points and all the researchers, students and staff in the Applied Mathematics Section for their friendship. I am also grateful to Sheffield Centre of Earth Observation Sciences and Engineering and Physical Sciences Research Council for funding my Ph.D. Finally, I wish to thank my parents and Sandry who encouraged me during this work. 
à mes parents 


\section{Table of Contents}

Chapter 1: Introduction 1

Chapter 2: HF radar measurements 9

2.1 What is an ocean wave? $\quad 10$

$2.2 \mathrm{HF}$ radar systems 10

\subsubsection{Concept 11}

2.2.2 OSCR system 12

$\begin{array}{ll}2.2 .3 \text { PISCES system } & 13\end{array}$

2.3 Doppler spectrum 14

2.3.1 Periodogram 14

2.3.2 Correlation coefficient 15

$\begin{array}{ll}\text { 2.3.3 Applications } & 18\end{array}$

$\begin{array}{ll}\text { 2.3.3.1 OSCR system } & 18\end{array}$

$\begin{array}{ll}\text { 2.3.3.2 PISCES system } & 18\end{array}$

2.4 First-order measurements 19

2.5 Second-order measurements $\quad 24$

2.5.1 Significant waveheight, $H_{S} \quad 26$

$\begin{array}{ll}\text { 2.5.2 Mean wave period, } T_{\omega} & 27\end{array}$

$\begin{array}{ll}2.6 \text { Data analysis } & 28\end{array}$

2.6.1 Complex normality 28

2.6.1.1 Normality 28

$\begin{array}{ll}\text { 2.6.1.2 Correlation } & 30\end{array}$

2.6.2 Wavelet analysis $\quad 30$

2.6.2.1 Non-stationarity signal analysis 31

2.6.2.2 The short-time Fourier transform : Analysis with fixed 32 resolution 
2.6.2.3 The continuous wavelet transform : a multiresolution analysis

2.6.2.4 Wavelet analysis and synthesis

2.6.2.5 Scalograms 36

2.6.2.6 Applications 36

Chapter 3: Modern spectral estimation

3.1 Historical review

3.2 Autoregressive spectral estimation

3.3 Autoregressive moving average spectral estimation

3.4 Eigenanalysis-based frequency estimation

3.5 Recursive MLE method

3.6 Summary

Chapter 4: The modified covariance method

4.1 Description

4.2 Fast algorithm to solve the modified covariance equations

of linear prediction

4.2.1 Special partitions of $\mathbf{R}_{p}$

4.2.1.1 Order-index partition

4.2.1.2 Time-index partition

4.2.2 Auxiliary parameters

4.2.3 Order update recursions

4.2.4 Time update recursions

4.2.5 Modified covariance algorithm $\quad 74$

4.2.5.1 Algorithm

4.3 Model order selection

4.4 Applications

4.4.1 OSCR system

4.4.2 PISCES system 
4.5.2 Solution . . . $\quad$. $\quad 84$

4.6 Summary $\quad . \quad 86$

Chapter 5: Methodology to compare the modified covariance $\quad 90$ method with the periodogram

5.1 Monte Carlo comparison 91

$\begin{array}{ll}\text { 5.1.1 Simulated data } & 91\end{array}$

5.1.2 Error measurements $\quad 93$

5.1.2.1 Spectral estimates $\quad 94$

5.1.2.2 Wave parameters 96

$\begin{array}{ll}\text { 5.1.3 Sample size problem } & 97\end{array}$

5.2 Wave-buoy comparisons 98

5.2.1 Description and locations 98

5.2.2 Error measurements $\quad 99$

5.2.2.1 "Sea truth" consideration $\quad 102$

$\begin{array}{ll}\text { 5.2.2.2 Symmetric regression } & 102\end{array}$

Chapter 6: Evaluation of the modified covariance method $\quad 104$ with the OSCR system

$\begin{array}{ll}6.1 \text { Good quality criteria } & 105\end{array}$

6.2 Monte Carlo results 105

$\begin{array}{ll}\text { 6.2.1 Spectral estimates } & 108\end{array}$

$\begin{array}{ll}\text { 6.2.2 Wave parameters } & 110\end{array}$

6.2.3 Summary $\quad 112$

6.3 Wave-buoy results 114

$\begin{array}{ll}\text { 6.3.1 Wind direction, } \theta_{\omega} & 115\end{array}$

6.3.2 "Sea-truth" consideration $\quad 115$

$\begin{array}{ll}\text { 6.3.3 Symmetric regression } & 119\end{array}$

6.4 Conclusion 120

$\begin{array}{ll}6.5 \text { Inversion procedure } & 120\end{array}$ 
Chapter 7: Evaluation of the modified covariance method with the PISCES system

7.1Wave-buoy results

7.1.1 Wind direction, $\theta_{\omega}$

7.1.2 "Sea-truth" consideration

7.1.3 Symmetric regression

Chapter 8: Conclusions

8.1 Modern spectral methods

8.2 Evaluation of the modified covariance method

8.2.1 Qualitative assessment

8.2.2 Quantitative assessment

8.3 Model order and data set length selections

8.4 Split peaks

8.5 Concluding remarks

Bibliography

Appendix A: Some corrected codes

A.1 Kolmogorov-Smirnov test

A.2 Procedure TEST 


\section{Glossary of abbreviations and symbols}

\section{Abbreviations}

$\begin{array}{ll}\text { AR } & \text { Autoregressive } \\ \text { ARMA } & \text { Autoregressive Moving Average } \\ \text { CWT } & \text { Continuous Wavelet Transform } \\ \text { FFT } & \text { Fast Fourier Transform } \\ \text { FMICW } & \text { Frequency Modulated Interrupted Continuous Wave } \\ \text { HF } & \text { High Frequency } \\ \text { OSCR } & \text { Ocean Surface Current Radar } \\ \text { MA } & \text { Moving Average } \\ \text { MSE } & \text { Mean Square Error } \\ \text { PSD } & \text { Power Spectral Density } \\ \text { RMLE } & \text { Recursive Maximum Likelihood Estimation } \\ \text { SNR } & \text { Signal-to-Noise Ratio } \\ \text { STFT } & \text { Short-Time Fourier Transform } \\ \text { WT } & \text { Wavelet Transform }\end{array}$

Symbols

$\begin{array}{ll}a & \text { scale factor } \\ a_{p} & \text { autoregressive parameters of a } p \text { th order AR process } \\ a_{p}^{f b} & \text { autoregressive parameters for the modified covariance method } \\ b_{q} & \text { moving average parameters of a } q \text { th order MA process } \\ \text { Bias(.) } & \text { bias } \\ c & \text { velocity of light } \\ \text { cor(.) } & \text { correlation coefficient } \\ C W T(\tau, a) & \text { continuous wavelet transform } \\ \chi^{2} & \text { chi-square distribution } \\ e_{p}^{f}(.) & \text { forward linear prediction error } \\ e_{p}^{b}(.) & \text { backward linear prediction error } \\ f & \text { frequency } \\ f_{b} & \text { Bragg frequency } \\ f_{d} & \text { Doppler shift } \\ f_{\text {radar }} & \text { radar frequency }\end{array}$




$\begin{array}{ll}\Delta f_{t} & \text { theoretical frequency difference } \\ g & \text { earth's gravity } \\ \gamma_{x x}(.) & \text { autocorrelation function } \\ H_{s} & \text { significant wave height } \\ \mathbf{I} & \text { identity matrix } \\ \mathbf{J} & \text { reflection matrix } \\ k & \text { ocean wavenumber } \\ k_{p} & \text { reflection coefficients } \\ \lambda & \text { radar wavelength } \\ M S E(.) & \text { mean square error } \\ \eta & \text { normalised Doppler frequency } \\ N & \text { data set length } \\ N\left(\mu, \sigma^{2}\right) & \text { normal distribution of mean } \mu \text { and variance } \sigma^{2} \\ v_{r} & \text { radial component of the target speed towards the radar } \\ p & \text { AR model order } \\ q & \text { MA model order } \\ r & \text { Bragg ratio } \\ r_{\text {overlap }} & \text { fractional overlap } \\ r_{P e a r s o n} & \text { Pearson's correlation coefficient } \\ r_{x x}(.) & \text { autocorrelation sequence } \\ R & \text { range } \\ \mathbf{R}_{p} & \text { autocorrelation matrix } \\ \rho_{p}^{f b} & \text { linear prediction error } \\ S(k, \theta) & \text { full directional spectrum } \\ S(f) & \text { true PSD function } \\ \hat{S}(f) & \text { estimate PSD function } \\ S_{e n}(f) & \text { energy spectrum } \\ S_{x}(f) & \text { periodogram } \\ S T F T(\tau, f) & \text { short-time Fourier transform } \\ \sigma^{+} & \text {integral under the positive Bragg peak } \\ \tau_{d} & \text { integral under the negative Bragg peak } \\ \tau_{\omega} & \text { normalised power spectrum of the second-order backscatter } \\ \sigma_{2}(\eta) & \text { sample interval } \\ & \text { mean period } \\ \text { time location } \\ T & \text { signal propagation delay } \\ & \end{array}$


direction of wave travel

propagation direction of the Bragg waves, along the radar beam towards from the radar

$\theta^{-}$ propagation direction of the Bragg waves, along the radar beam away from the radar

$\theta_{\omega}$ wind direction

$u($.

white noise process

Var(.) variance

$x($.

time series 


\section{Chapter 1}

\section{Introduction}

High-Frequency (HF) radar systems have the unique ability to picture the evolution of wave dynamics over a wide sea area under any weather conditions. The wave measurements are obtained from the HF radar by inverting a non-linear integral equation describing the relationship between the power spectrum of the radar backscatter and the ocean wave directional spectrum. This power spectrum is known as the Doppler spectrum.

The present techniques for obtaining the Doppler spectrum have a major shortcoming: they require too long data sets. This is not acceptable for two reasons. First, the seastate can only be considered stationary in sufficiently short time periods, shorter than the ones presently used. Second, the measurements can be subject to wind and wave variabilities.

In this thesis, we solve this problem by looking at new spectral techniques. These methods work with shorter data sets.

The work at Sheffield on HF radar remote sensing aims to measure the ocean wave directional spectrum over large areas of the coastal ocean. This spectrum contains information on both locally-generated wind waves and swell components from distant storms. In fact, the HF radar system measures backscatter from the ocean surface, in other words the moving waves, as well as from anything else such as ships. This backscatter is converted to the Doppler spectrum, which leads to current and wave measurements. The quality of the Doppler spectrum is responsible for the accuracy of these wave measurements. Thus, it is necessary to use a spectral estimation method which gives the best quality of this spectrum.

Figure 1.1 shows a typical Doppler spectrum. Barrick (1972) developed two strong mathematical theories to describe this spectrum. The first-order theory represents the two distinct peaks (see label $\mathrm{A}$ in figure 1.1) and this provides current measurements. 
The continuum surrounding these two peaks is the second-order theory (see label B in figure 1.1). Wave measurements are extracted from this contribution.

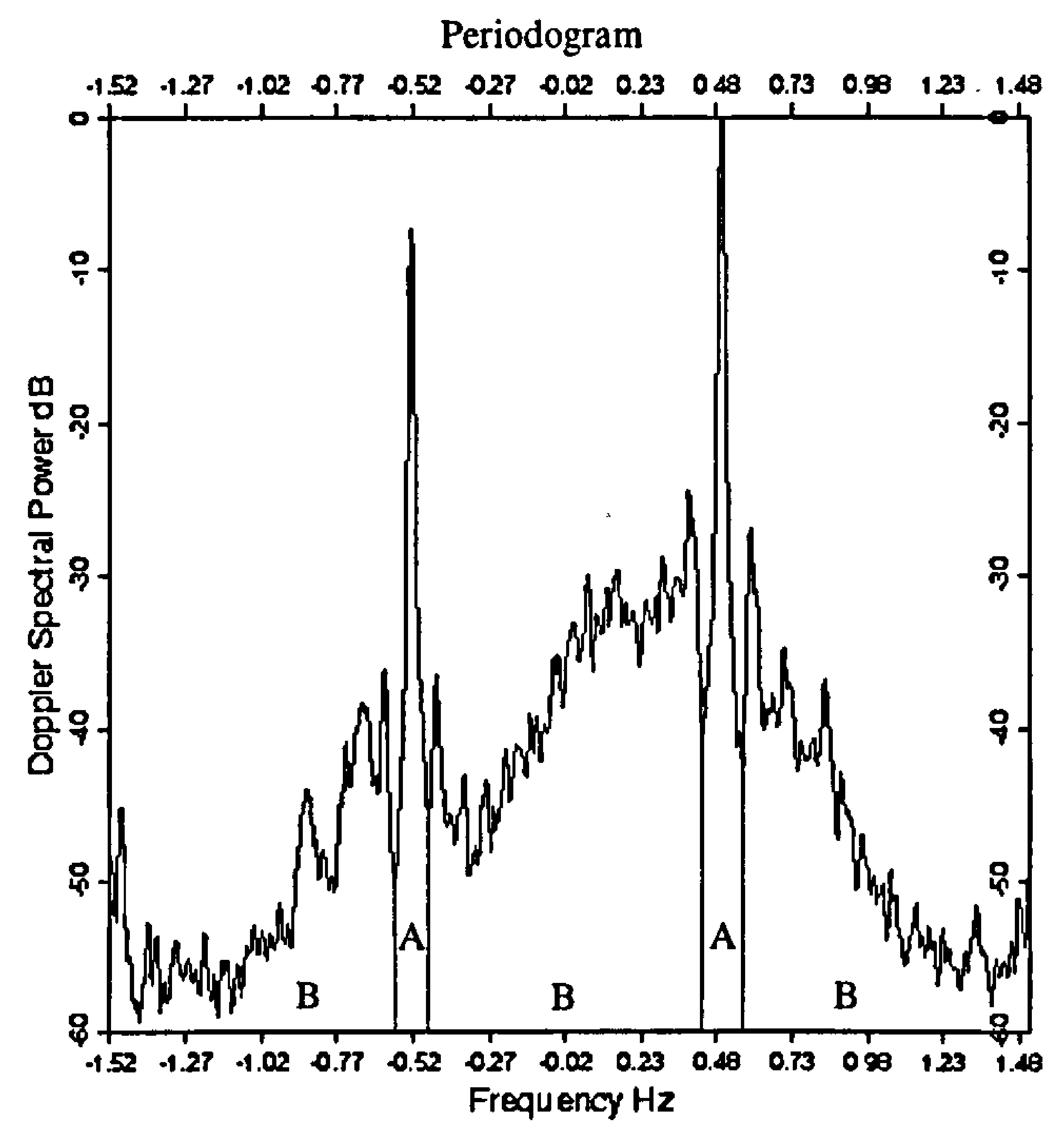

Figure 1.1: Typical Doppler spectrum showing (A) first- and (B) second-order contributions.

The usual spectral technique used to produce the Doppler spectrum is the periodogram (Harris 1978). This is based on the averaging of several spectra obtained by the Fast Fourier Transform (FFT), which is a fast algorithm applying the Fourier transform to the signal. This method will be described in the next chapter.

In order to get wave measurements, it is essential to reduce reasonably the variance in the Doppler spectrum. This is achieved with the periodogram by averaging a certain number of Doppler spectra, which leads to the necessity of using long data series. Therefore, non-stationarity present in the sea state can become a major problem for wave measurements. If the current varies considerably, this can lead to a smearing of the first-order peaks and then prevent the separation of the first- and second-order parts of the spectrum which is necessary to get the ocean wave directional spectrum. Figure 1.2 illustrates this kind of problem and shows split peaks. One possible way to overcome this problem is to reduce the averaging process and therefore use shorter 
data collection times, hence the need for alternative spectral analysis methods.

PERIODOGRAM

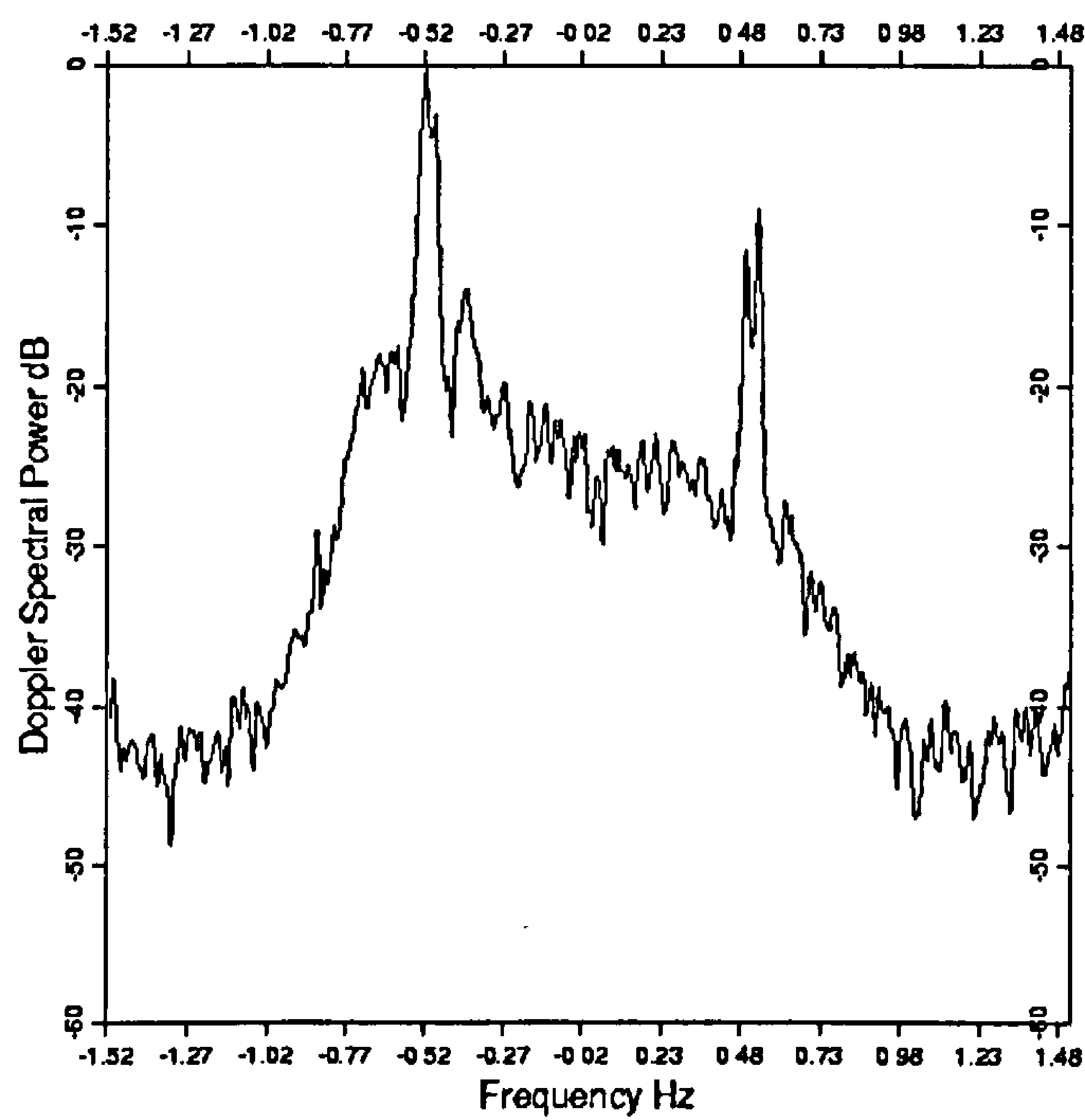

Figure 1.2: Doppler spectrum showing split peaks.

When the sea conditions are varying very quickly, the measurement period may not be sufficiently short to get enough information about the different sea states. This is the case for some measurements of the Petten experiment in the SCAWVEX program (Wyatt, 1997d). This experiment was carried out on the Dutch North Sea coast at Petten (see figure 1.3). The measurements were conducted during the storm season (from November to December 1996) and originated from (with a twenty-minute cycle) two HF radars (WERA systems), represented as $M$ and $S$ in figures 1.4, 1.5 and 1.6. As can be seen in these figures, in some parts of the sea region covered (for example, along longitude 4.40), the wind direction (shown by arrows) and the significant wave height (shown by shadows) change considerably during this period of time. Therefore, one cannot be sure that twenty minutes is short enough to measure wind and hence wave variations, which is another motivation to work with short data collections. Also, it is obvious that this problem may occur when the data collections 
are longer, for example, in the case of the OSCR system (another HF radar system which will be introduced later on) where these collections take place over one hour. The objective of this work is to develop a spectral estimation method which allows us to use short time data collection.

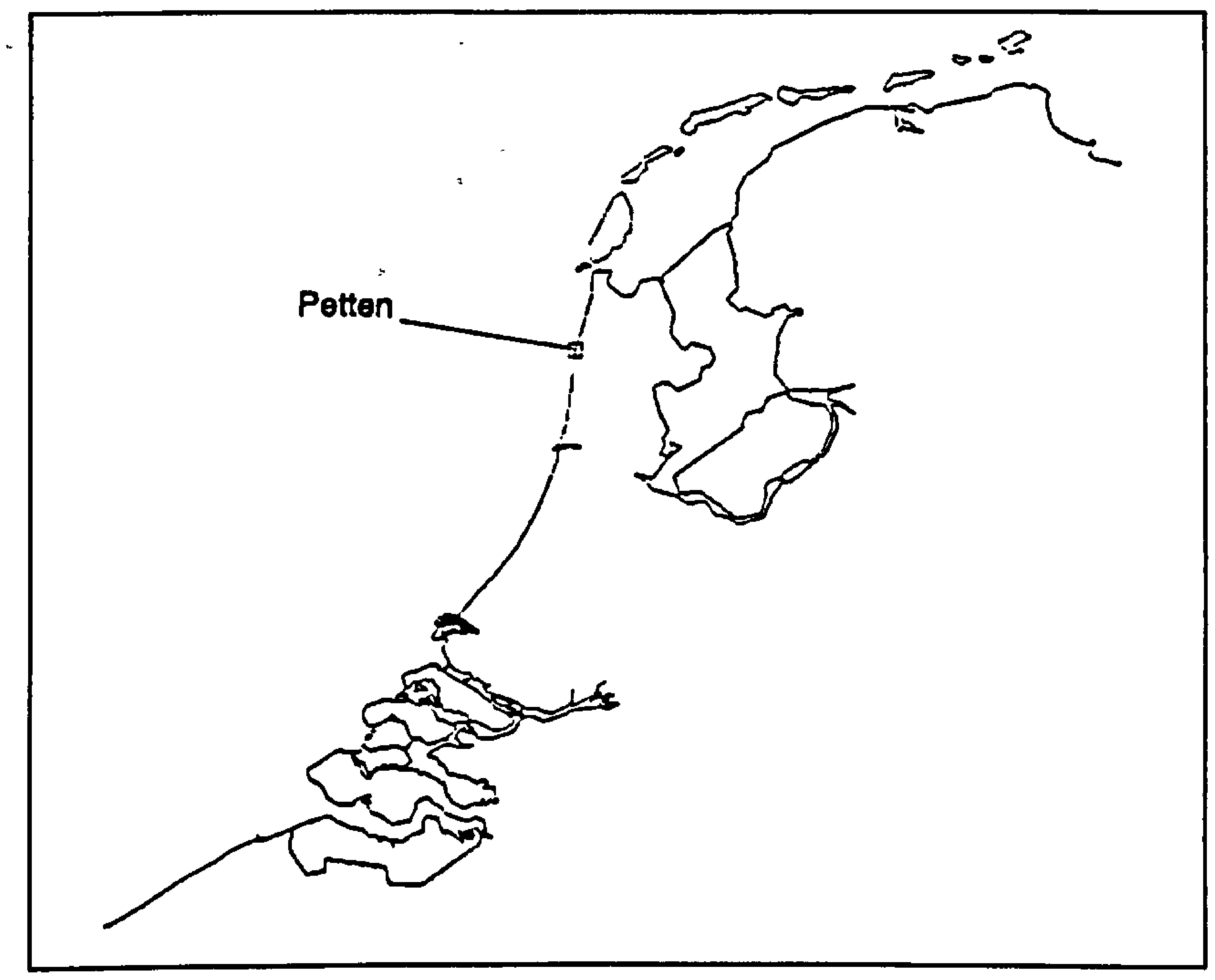

Figure 1.3: Measurement location of the Petten experiment.

The modern spectral estimation methods have the potential, when used with HF radar data, of increasing the temporal resolution in current measurements thus allowing the monitoring of rapidly varying oceanographic conditions. Kahn (1991) showed that the ocean clutter can be modelled with two narrowband Bragg signals with time-varying frequencies and postulated that the time variations of the Bragg frequencies are related to ocean wave height. For estimating the radial component of surface current flow from HF radar measurements (Martin and Kearney, 1997), autoregression analysis is intrinsically more accurate than the FFT technique with short data sets. But nothing has been done concerning wave measurements. This thesis deals not only with current measurements but also the use of such techniques is investigated in wave measurements. In another words, the second-order part of the Doppler spectrum becomes very important in this research. 


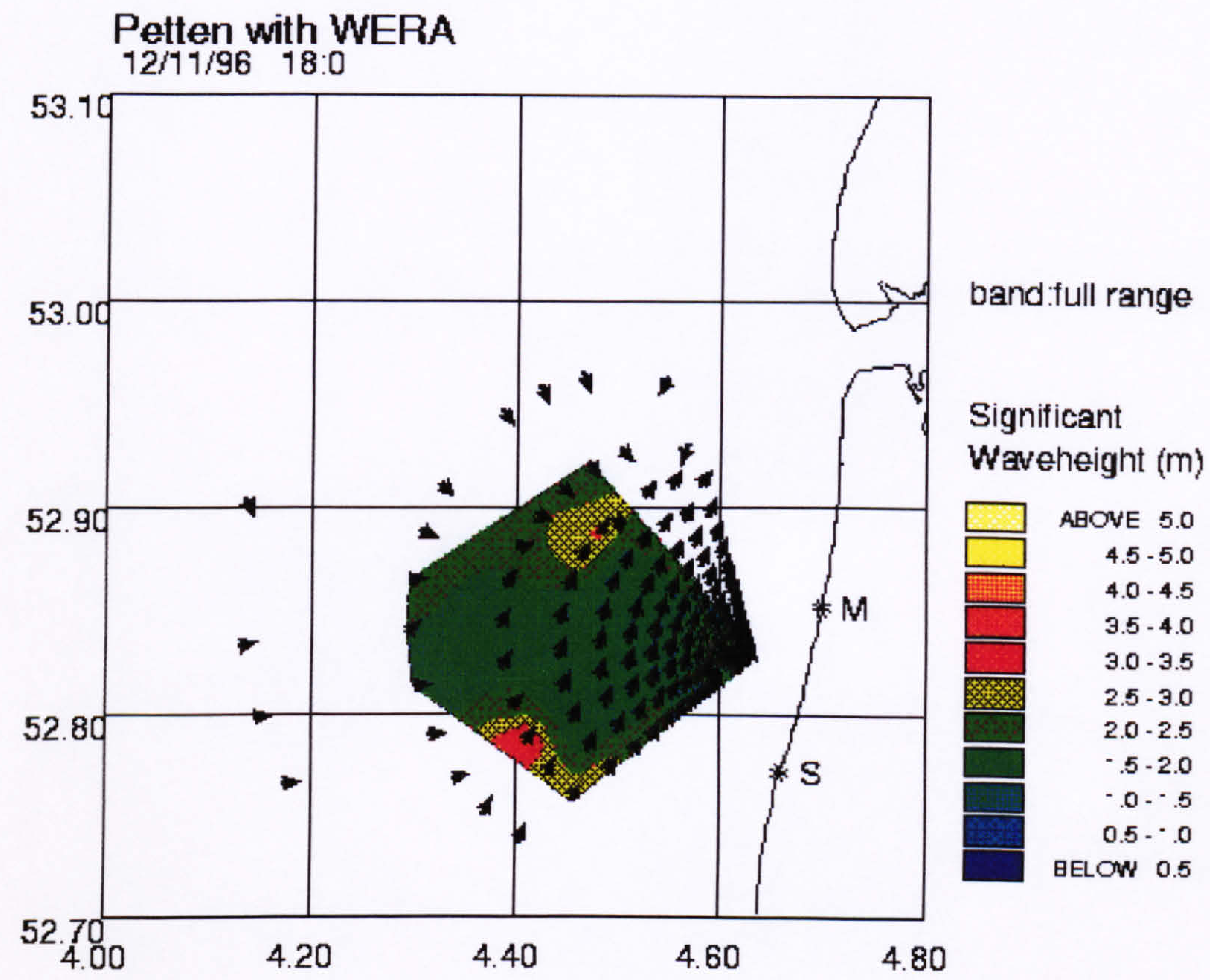

Figure 1.4: HF radar coverage showing wind measurements (by arrows) and wave measurements (within shaded area). These measurements were taken at $6 \mathrm{pm}$ on 12 November 1996. 


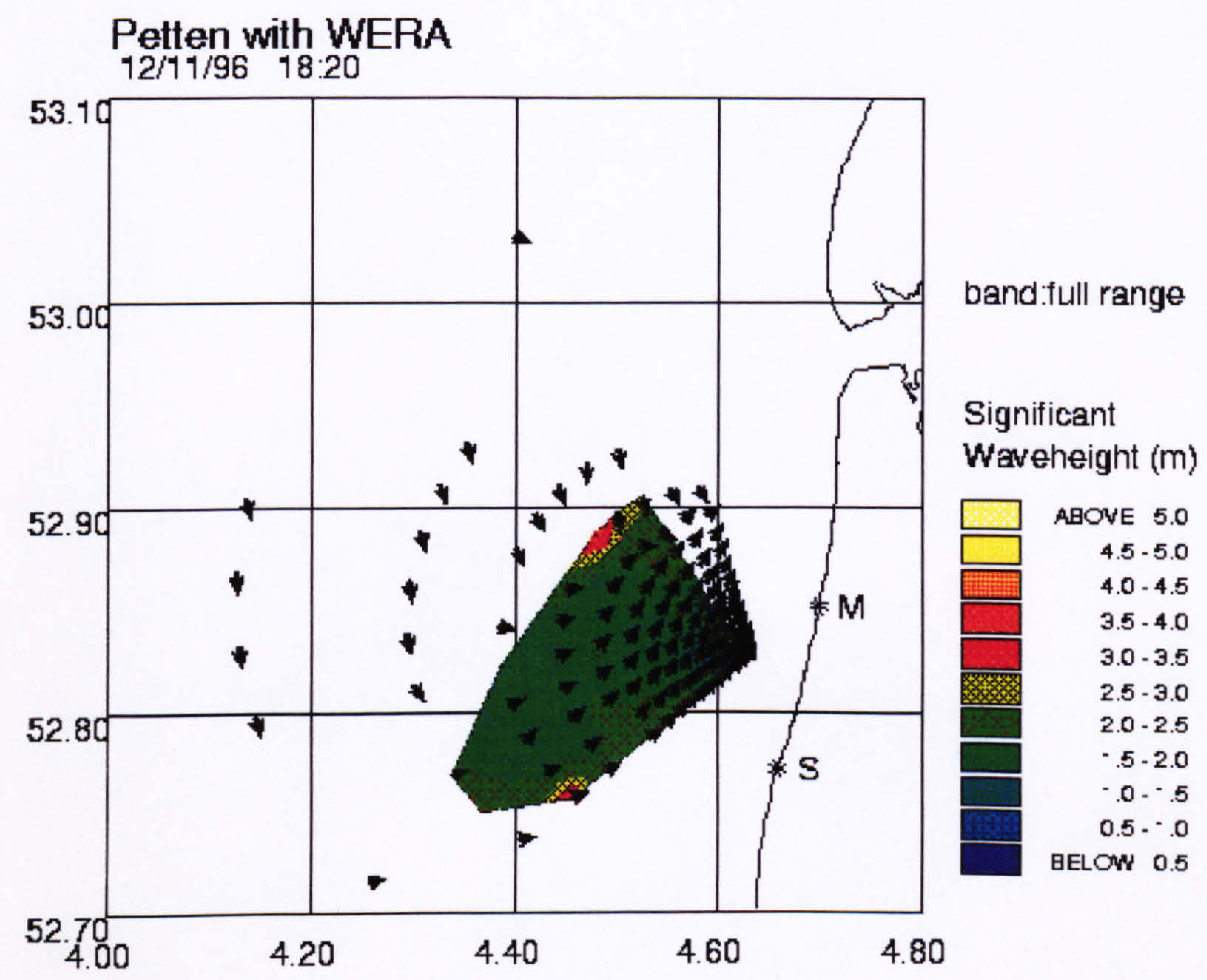

Figure 1.5: HF radar coverage showing surface wind measurements (by arrows) and wave measurements (within shaded area). These measurements were taken at 6:20pm on 12 November 1996. 


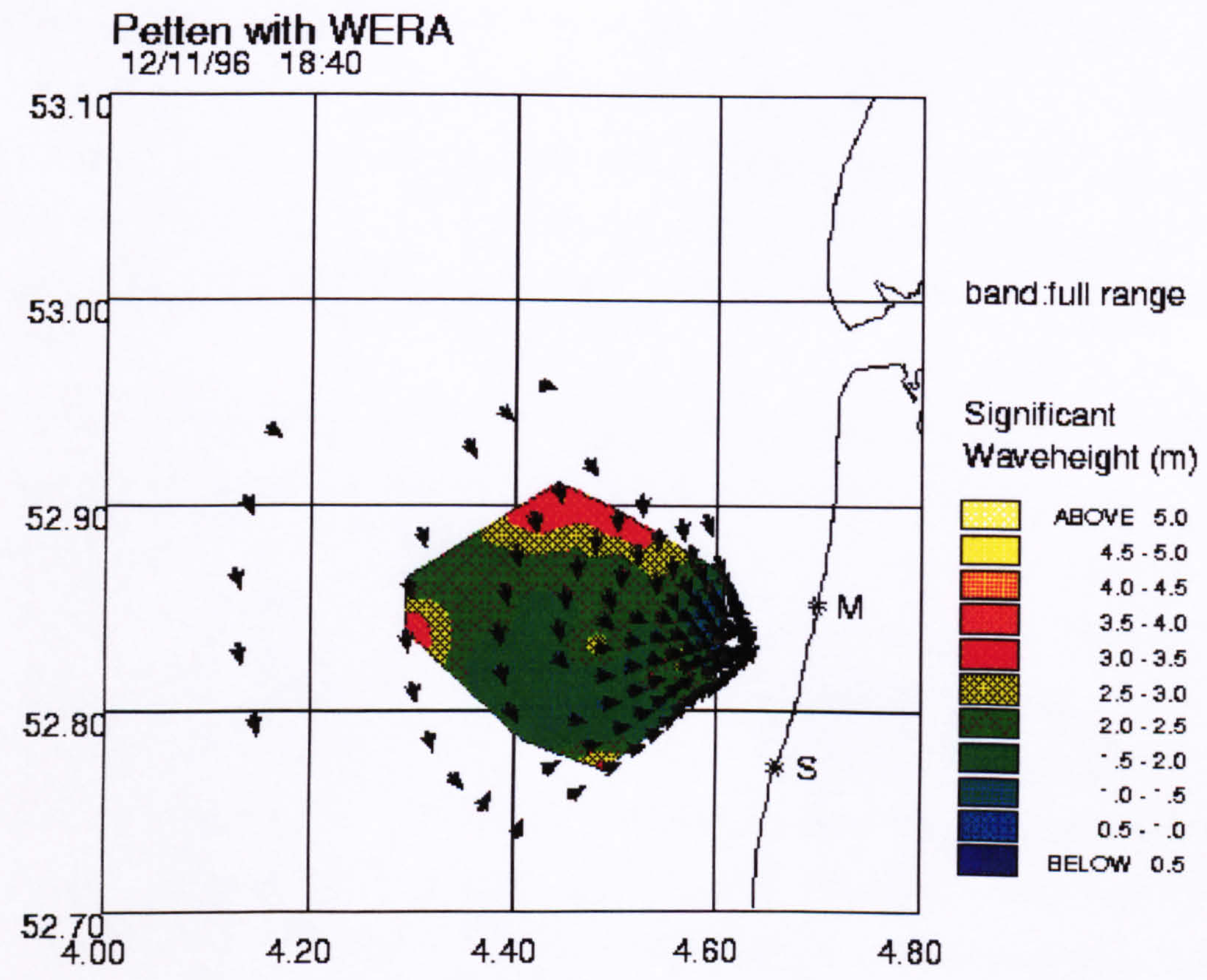

Figure 1.6: HF radar coverage showing surface wind measurements (by arrows) and wave measurements (within shaded area). These measurements were taken at 6:40pm on 12 November 1996. 
Chapter 2 of this thesis is concerned with HF radar measurements. Two HF radar systems other than the WERA system are presented: the PISCES and OSCR systems, which supply data for this work. The standard technique for producing the Doppler spectrum, the periodogram, is described. Since wave measurements are obtained from the Doppler spectrum, we give a detailed description of the first- and second-order parts of this spectrum and present these wave parameters. Also, the statistics of the backscattered data are discussed because these will be useful in the following chapters.

Chapter 3 provides a review of modern spectral analysis methods. Four different spectral estimations are presented and applied to HF radar systems: 1. the autoregressive spectral estimation suitable for spectra with sharp peaks; 2 . the autoregressive moving average spectral estimation able to represent different spectral shapes; 3. the eigenanalysis-based frequency estimation producing peaks of narrowband spectra and 4- the recursive maximum likelihood estimation (RMLE) which is restricted to real data. One spectral analysis method is selected according to the nature of the HF radar Doppler spectrum with its dynamic range and distinct peaks. This is the modified covariance method based on the autoregressive spectral estimation.

Chapter 4 describes the modified covariance method and points out its major problem, the selection of the model order. As well, this chapter defines the problem of split peaks as mentioned above and emphasizes the importance of using the modified covariance method.

In order to validate the modified covariance method, we need to compare it with the periodogram. To do so, two methods of comparison are introduced in Chapter 5. One is based on the Monte Carlo simulation study, which evaluates the performance of the different spectral methods. The second uses wave-buoy measurements. By extracting the wave parameters from the different spectral estimates, we can compare these quantities with the wave-buoy parameters.

The results obtained from these comparisons are presented in Chapters 6 (for the OSCR system) and 7 (for the PISCES system).

The conclusion of this work is in Chapter 8. 


\section{Chapter 2}

\section{HF Radar Measurements}

Radio oceanography was first introduced during World War II. The objective was to provide a radar map of the surrounding ocean in order to get ship locations and was not to measure waves. At the time, sea echoes from the ocean were undesirable and this is why people called it "clutter". However, this phenomenon was of great interest for scientists who wanted to measure waves and understand the mechanisms behind it. It was Crombie (1955) who first established the relationship between the scattering of coherent $\mathrm{HF}$ radar (operating at frequencies $3-30 \mathrm{MHz}$ ) and waves on the ocean surface. Since then, HF radar systems have been developed and become a powerful tool for remote sensing of the sea-state.

Two HF radar systems (OSCR and PISCES) are described in this chapter. We also present the Doppler spectrum and its characteristics, since the spectrum is essential to obtain both current and wave measurements. Finally, the nature of the HF radar data is statistically analyzed in order to apply some methods in the following chapters. 


\subsection{What is an ocean wave?}

HF radar systems are used to provide measurements of the ocean waves.

Wave motion is carried out by particles of water exhibiting circular motion as the wave travels. At the surface of the sea, these circular motions can have different amplitudes. Waves are generated by the wind either by distant storms or locally. The waves created by the distant storms are known as "swell". Having long wavelengths, they can travel long distances with low attenuation. The locally driven waves are more complex. When the wind blows over the sea, short-wavelength waves, called "wind-sea", are formed. As these build up, the energy is transferred into longer waves with larger amplitudes.

\subsection{HF radar systems}

HF radar systems are used for remote sensing of the sea-state (Paduan and Graber, 1997). They provide monitoring of ocean surface waves and current over a wide surface area of the sea.

A transmitted radio wave is scattered from any surface of ocean with which it interacts. This backscatter is received at the radar which measures the time and phase differences between the transmitted and received waves. The resultant power spectrum, referred to as the Doppler spectrum, which is based on the FFT, is computed. This spectrum is essential to get measurements about the ocean waves.

Two HF radar systems are used for this work: the PISCES (not an acronym, c.f. OSCR) system, intended for long range measurement of both current and waves, and the OSCR (Ocean Surface Current Radar) system, a short range high resolution system, developed for coastal current measurements. In order to eliminate directional 
ambiguities, both the OSCR and PISCES systems comprise two radars positioned a distance apart from each other along the coast (Wyatt, 1987).

\subsubsection{Concept}

Radar stands for Radio Detection and Ranging. The radars have a principal radio frequency, the carrier, set by a frequency synthetizer.

For a pulse radar (e.g. the OSCR system as described below), this continuous signal is pulsed on and off by an modulator. The short bursts of radio energy that result are amplified by a transmitter and sent to the antenna via a switch. The radio wave travels away from the radar at the speed of light, is scattered from a target and returns to the radar.

The range, $R$, of the target is computed from the signal propagation delay $\tau_{d}$ by

$$
R=c \tau_{d} / 2
$$

where $c \approx 3 \times 10^{8} \mathrm{~m} / \mathrm{s}$ is the velocity of light, the velocity at which the radio wave travels.

The wave length of the radio wave, $\lambda$, is related to the frequency, $f$, by

$$
\lambda=c / f
$$

The change in the frequency of the radio signal caused by the motion of the target is called Doppler shift. This shift is related to the velocity of target by

$$
\Delta f=f_{d}=2 v_{r} / \lambda
$$

where $v_{r}$ is the radial component of the target speed towards the radar. The Doppler shift was named after C.J Doppler (1803-1853). He pointed out that the color of a luminous body and the pitch of a sounding body are changed by the relative motions of the body and the observer.

The radio wave frequencies that range from 3 to $30 \mathrm{MHz}$ are classified as high frequencies, HF. These HF radio waves have two alternate modes by which they can reach surface points beyond the horizon. The first involves diffraction by the curved earth; at HF, a vertically polarised wave can be diffracted a considerable distant beyond the horizon due to the much longer wavelength and the highly conductive 
properties of sea water at these frequencies. This mode of radio propagation is referred to as the "ground-wave" or "surface wave" mode. The second mode of HF propagation, referred to as "skywave", results from the presence of the ionosphere above the earth. Sea echo has been observed by both these modes.

\subsubsection{OSCR system}

The OSCR system (Wyatt 1994 and Wyatt and Ledgard 1996), has been developed to provide solutions to coastal engineering problems, such as erosion problems or sandbank movements.

As a pulse radar, this system uses a $\mathrm{HF}(25.4-27 \mathrm{MHz})$ radio frequency to map surface current patterns over a large area of the ocean. It is a land based portable radar system and consists of two radars, termed the master and slave, placed a distance apart from each other. For the work discussed in this thesis, experiments were conducted along the Holderness coastline (Prandle et al., 1996) and the radar sites were approximately $15 \mathrm{~km}$ apart from each other. In order to avoid the signals interfering with each other, each unit makes independent measurements sequentially. These measurements were taken at up to 559 cell positions with a range extending to $40 \mathrm{~km}$ offshore with a cell resolution of $1 \mathrm{~km}^{2}$. As a pulse radar, range gating is carried out in time domain. A single FFT provides the power spectrum at each range. It collects data for just five minutes over a twenty-minute cycle. At present, three separate five-minute data sets have to be averaged in order to obtain stable spectral estimates for wave measurements (current measurements are made using the five-minute data), thus measurements represent an hourly average.

Since these measurements have been collected in coastal regions they may be subject to variations on time scales that are often less than one hour, leading to uncertainty in the interpretation of the data. This problem was already mentioned in the Introduction with the Petten experiment. 


\subsubsection{PISCES system}

The PISCES system was used to monitor waves and currents in the Celtic sea area. Two radars were set up. One was located on the South Wales coast, the other one on the North coast of Devon (Wyatt, 1995).

PISCES is not a pulse radar but a FMICW (Frequency Modulated Interrupted Continuous Wave) radar; it transmits and receives a sweep in frequency (from frequency $f_{1}$ to frequency $f_{2}$ ), repeated for the duration of the measurement. (The WERA system mentioned in Chapter 1 is a FMCW system. The difference occurs in the use of the transmitter and receiver.) For the FMICW radar, the receiver is switched off when the transmitter is working and vice-versa. Each sweep is processed using a FFT, the frequency bins of which identify the phase and amplitude of backscatter from consecutive range bins. The range resolution is determined by the total frequency difference, $f_{1}-f_{2}$, typically twenty $\mathrm{kHz}$ giving a resolution of $\frac{c}{\left(f_{1}-f_{2}\right) * 2}=7.5 \mathrm{~km}$. Consecutive sweeps then contribute to a time series of backscatter for each range cell. A second FFT is required to provide the power spectrum of backscatter at that range. This second stage is identical to the processing which is employed by pulse radars (such as the OSCR system as described above), providing complex samples for each range bin.

To obtain stable estimates, taking account the random nature of the sea surface, a certain amount of averaging of the second FFT is necessary. Therefore, about three minute FFTs averaged over about 30 minutes have been used. As has been said in Chapter 1 , this can lead to problems in very variable sea conditions, particularly if measurements are required over a large area. 


\subsection{Doppler spectrum}

To get the ocean parameters, we compute the Doppler spectrum from the time series data obtained from the different HF radar systems. The method, usually used to produce the Doppler spectrum, is the well-known periodogram (Marple, 1987 and Harris, 1978).

\subsubsection{Periodogram}

The periodogram consists of segmenting the data into overlapping segments and averaging the sample spectrum of each segment.

Assume a complex stationary data set $x(0), \ldots, x(N-1)$ of $N$ data points, which is divided into $P$ segments of $M$ samples each. Each segment is shifted with a shift $Q$ (see figure 2.1).

For both systems, we have taken $M=512$ and $Q=128$. Hence the overlap is $\frac{512-128}{512}=75 \%$.

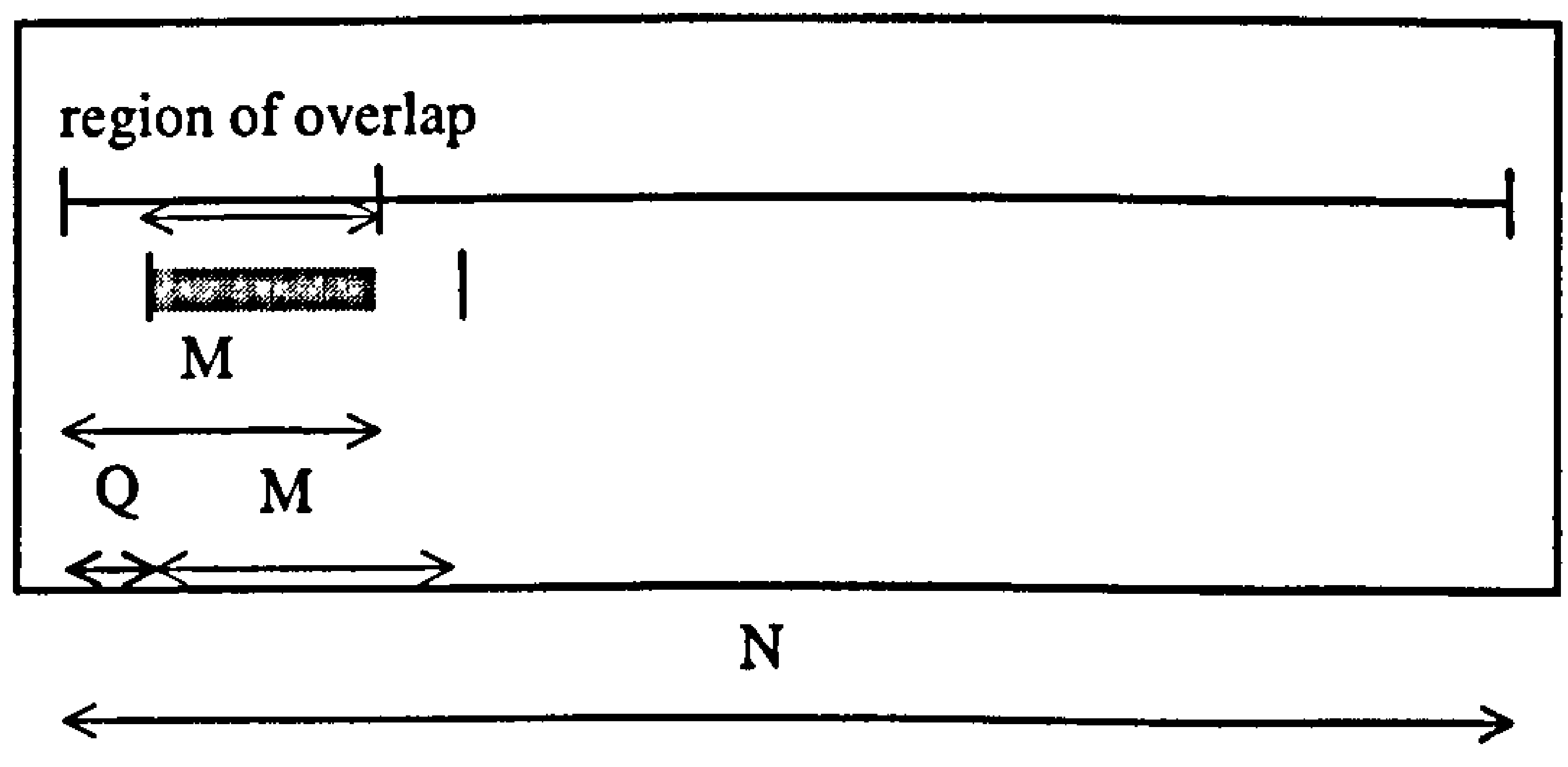

Figure 2.1: Partition of segments for overlapped processing. 
The purpose of overlapping segments is to increase the number of segments in order to decrease the variance of the power spectrum density (PSD) estimate (see next section).

In order to reduce the effect of sidelobes (spurious peaks surrounding the main lobe) and decrease the estimation bias, a window is applied in each segment before computing the sample spectrum. Thus the $p^{\text {th }}$ segment consists of the samples:

$$
x^{(p)}(m)=w(m) x(m+p Q), \begin{gathered}
0 \leq m \leq M-1 \\
0 \leq p \leq P-1
\end{gathered}
$$

where $w(m)=0.35875-0.48829 \cos \left(\frac{2 \pi m}{M}\right)+0.14128 \cos \left(\frac{4 \pi m}{M}\right)+0.01168 \cos \left(\frac{6 \pi m}{M}\right)$ is the Blackman-Harris window (minimum 4-sample). From the range of the windows available, this window is recommended by Harris (1978) because of its efficiency in reducing spectral leakage and because it has the lowest correlation coefficient (see next section) for a $75 \%$ overlap.

Then, the sample spectrum of the $p^{\text {th }}$ segment is

$$
S_{x}^{(p)}(f)=\frac{1}{T M U}\left|X^{(p)}(f)\right|^{2},-1 / 2 T \leq f \leq 1 / 2 T
$$

where $T$ is the sample interval, $U=T \sum_{m=0}^{M-1} w^{2}(m)$ is the discrete-time window energy, and $X^{(p)}(f)=T \sum_{m=0}^{M-1} x^{(p)}(m) e^{-2 i n f m t}$ is the discrete Fourier transform.

The quantity $U$ is present to remove the effect of the window energy bias in the final PSD estimate, the periodogram:

$$
S_{x}(f)=\frac{1}{P} \sum_{p=0}^{p-1} S_{x}^{(p)}(f), \quad-1 / 2 T \leq f \leq 1 / 2 T .
$$

\subsubsection{Correlation coefficient}

The correlation coefficient shows the correlation existing between 2 successive segments and the window efficiency. It is defined by 


$$
\operatorname{cor}\left(r_{\text {overlap }}\right)=\frac{\sum_{m=0}^{M-1} W(m) W\left(m+\left(1-r_{\text {overlap }}\right) M\right)}{\sum_{m=0}^{M-1} W^{2}(m)}, \text { for } 0 \leq r_{\text {overlap }}<1 \text {, }
$$

where $r_{\text {overlap }}=\frac{M-Q}{M}$ is the fractional overlap and $W(m)$ is the window Fourier transform.

When $P$ segments are averaged, the variance of the average, $\sigma_{\text {AVERAGE }}^{2}$, is related to the individual variance of the segments, $\sigma_{S E G M E N T}^{2}$, (Harris 1978), for a 75\%-overlap, by:

$$
\frac{\sigma_{A V E R A G E}^{2}}{\sigma_{\text {SECMENT }}^{2}}=\frac{1}{P}\left\{1+2 \operatorname{cor}^{2}(0.75)+2 \operatorname{cor}^{2}(0.5)\right\}-\frac{2}{P^{2}}\left\{\operatorname{cor}^{2}(0.75)+2 \operatorname{cor}^{2}(0.5)\right\}(2.8)
$$

If there is no overlapping, then $\frac{\sigma_{A V E R A G E}^{2}}{\sigma_{S E G M E N T}^{2}}=\frac{1}{P}$.

Also, we have $\frac{\sigma_{A V E R A G E}^{2}}{\sigma_{S E G M E N T}^{2}}=\frac{2}{\mu}$ with $\sigma_{A V E R A G E}^{2}=\frac{1}{\mu}$ and $\sigma_{S E G M E N T}^{2}=\frac{1}{2}$.

$\mu$ is the degree of freedom of the "averaged" periodogram. The degree of freedom of the periodogram for each segment without overlapping (Priestley, 1981) is 2.

This last formula is due to the distribution of the periodogram. Assuming that the initial process is a Gaussian purely random process, Priestley (1981) proved that the periodogram follows a $\chi^{2}$ distribution. This property of normality will be shown later in section 2.6.1.

In our case, using the Blackman-Harris window (minimum 4-sample), the different values taken by the correlation coefficient are: $\operatorname{cor}(0.75)=0.46$ and $\operatorname{cor}(0.5)=0.038$.

Hence, with the PISCES system, we have

- $N=4608, M=512, Q=128, P=33 \Rightarrow \frac{\sigma_{\text {AVERAGE }}^{2}}{\sigma_{\text {SECMENT }}^{2}}=0.043$ in the case of overlapping,

- $N=4608, M=512, Q=128, P=9 \Rightarrow \frac{\sigma_{A V E R A G E}^{2}}{\sigma_{S E G M E N T}^{2}}=0.11$ if we do not overlap. 
And for the OSCR system, we need to combine the equations (2.8), (2.9) and (2.10). For each segment of 896 data points, we overlap 4 segments of 512 points and obtain a "first" periodogram. Then, we average these 3 "first" periodograms in order to produce the final periodogram.

- $N=2688, M=512, Q=128, P=4 \times 3=12 \Rightarrow \frac{\sigma_{\text {AVERAGE }}^{2}}{\sigma_{\text {SEGMENT }}^{2}}=0.11$ in the case of overlapping,

- $\quad N=2688, M=512, Q=128, P=3 \Rightarrow \frac{\sigma_{A V E R A G E}^{2}}{\sigma_{S E G M E N T}^{2}}=0.333$ if we do not overlap.

- $N=896, M=512, Q=128, P=4 \Rightarrow \frac{\sigma_{\text {AVERAGE }}^{2}}{\sigma_{\text {SEGMENT }}^{2}}=0.33$ in the case of overlapping one segment of 896 point data.

Therefore, overlapping is important to decrease the PSD estimate variance. The variance reduction with the OSCR system is limited by the operational constraint, so that we cannot do as well as with the PISCES system. As can be seen with the OSCR system, the fact of averaging 4 segments produced from the 5-minute collection period is not sufficient to reduce the PSD estimate variance. This is the reason that 3 separate 5-minute data sets are averaged and overlapped in order to obtain satisfactory quality signal to noise for wave measurements. We could extend averaging over longer periods, but this option is not acceptable. Non-stationarity is to be avoided as far as possible. The PSD estimate variance is used to determine the confidence intervals for wave measurements (Wyatt 1991). 


\subsubsection{Applications}

\subsubsection{OSCR system}

Figure 2.2 illustrates a periodogram obtained from the OSCR system. We recall that a raw file contains 3 segments of 896 samples, in other words 2688 data points. Each segment represents one 5-minute measurement and is separated by 20 minutes, so that it corresponds roughly to one hour measurement. For each segment, using a 75\%overlap, 4 segments, each of 512 samples, have been overlapped and averaged. Then the 3 resulting segments are averaged. In total, 12 segments have been used.

\subsubsection{PISCES system}

Briefly, the PISCES system provides files of 4608 data samples, this corresponds to a 30-minute measurement cycle. In order to obtain the periodogram (figure 2.3), 33 segments (of 512 samples) have been used, also using a 75\%-overlap.

As it can be seen, the fact that the variance is more reduced for this system makes the periodogram in figure 2.3 look "smoother" than the one obtained with the OSCR system shown in figure 2.2 .

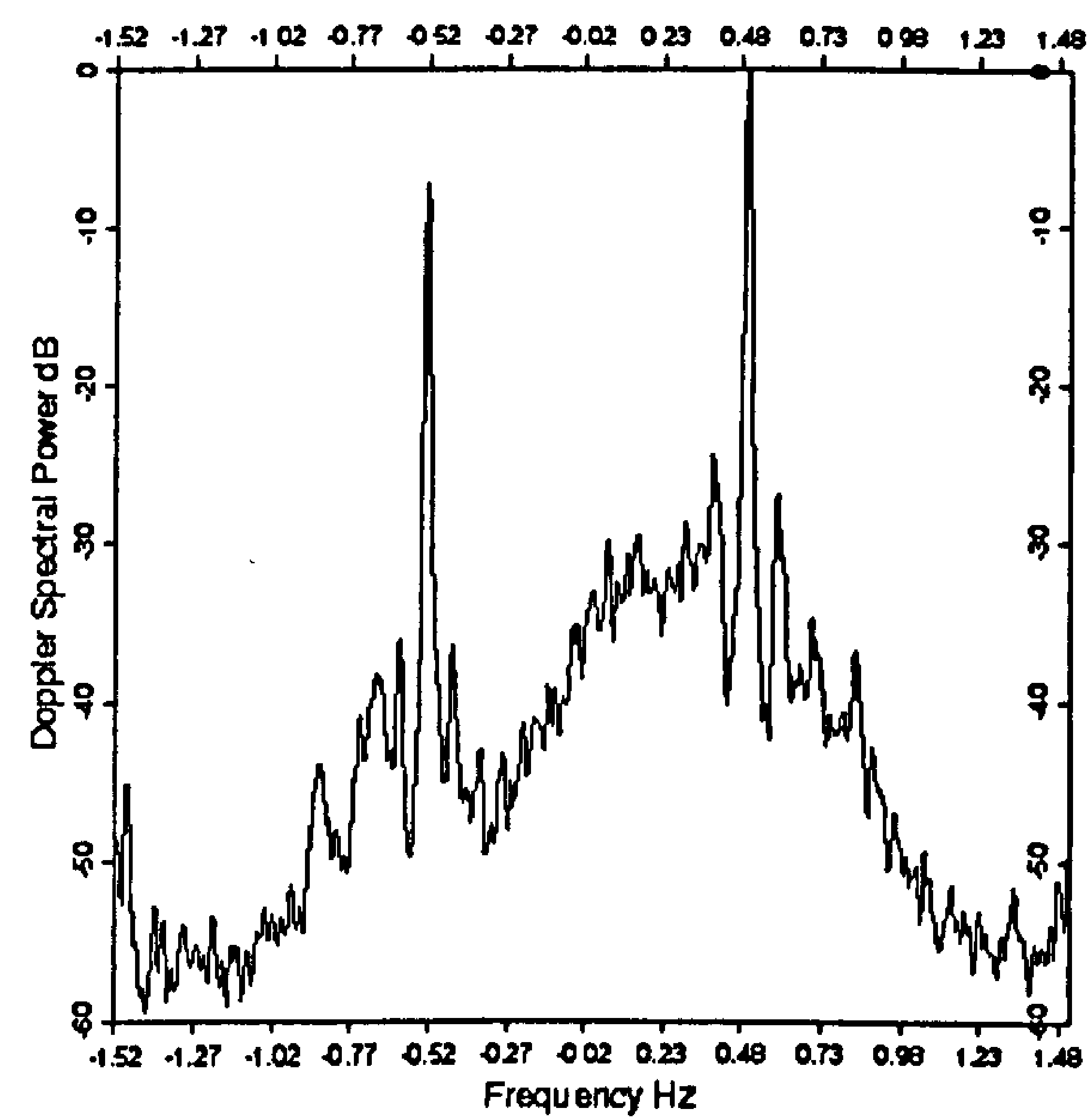

Figure 2.2: Periodogram from the OSCR system (measurement taken at $3 \mathrm{pm}$ on 27 December 1995), $N=2688$ and $P=12$. 


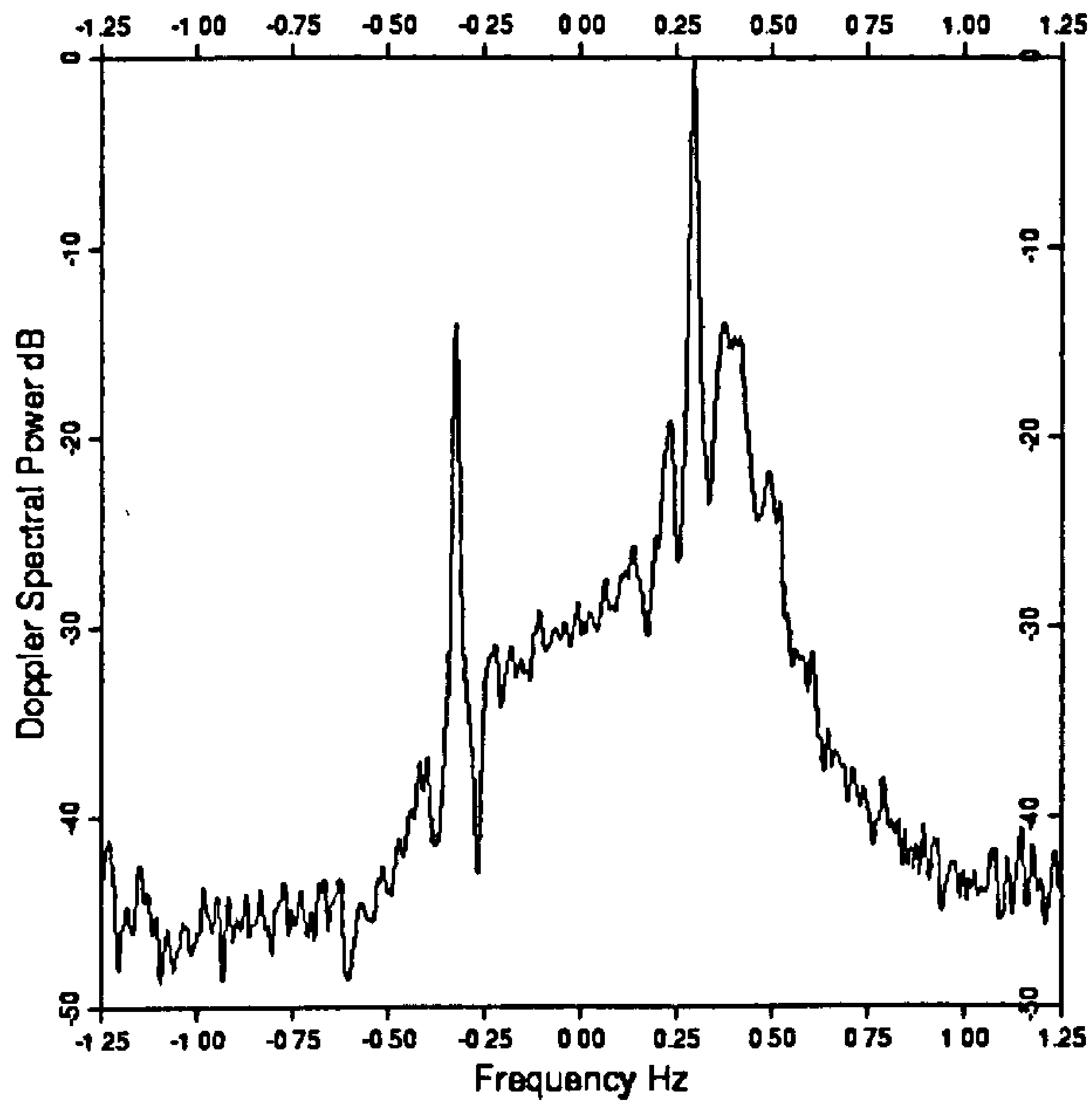

Figure 2.3: Periodogram from the PISCES (measurement taken at 8a.m. on $28 \mathrm{March}$ 1987), $N=4608$ and $P=33$.

\subsection{First-order measurements}

Figure 2.4 shows a typical Doppler spectrum obtained from the OSCR system. It was Crombie (1955) who deduced the Bragg scatter mechanism or first-order effect characterized by two prominent peaks symmetrically spaced about the carrier frequency (Forget et al., 1981 and Wyatt, 1990a), represented by A in figure 2.4. This mechanism was seen to be "Bragg scatter", the same phenomenon responsible for scatter of $\mathrm{X}$-rays in crystals and light rays from diffraction gratings.

The ocean wave-trains present on the sea interact with the radar wave. The sea-waves of wavelength $\lambda / 2,2 \times \lambda / 2,3 \times \lambda / 2 \ldots$ where $\lambda$ is the radar wavelength, backscatter almost all the energy toward the radar. Those having wavelengths equal to $\lambda / 2$, and moving toward (positive Doppler frequency) and away from (negative Doppler frequency) the radar, give the strongest echo (Barrick, 1977). This is illustrated in figure 2.5. For such waves, the dispersion relation linking wavelength $\lambda$ to wave 
velocity is $v= \pm \sqrt{g \lambda / 4 \pi}$ where $g$ is the earth's gravity (Tucker 1991). When there is no surface current, the peaks are located at the Doppler frequencies $\pm f_{b}$, with $f_{b}= \pm \frac{2 v}{\lambda}= \pm \sqrt{\frac{g f_{\text {radar }}}{\pi c}}, f_{\text {radar }}$ being the radar frequency (see section 2.1.1). In the presence of a surface current, the peaks are at frequencies $f_{u} \pm f_{b}$, where $f_{u}=\frac{2 v_{r} f_{\text {radar }}}{c}\left(v_{r}\right.$ being the radial component of surface current towards the radar $)$ is a frequency shift proportional to the magnitude of the current. Determining this shift is the principle behind measuring the surface current.

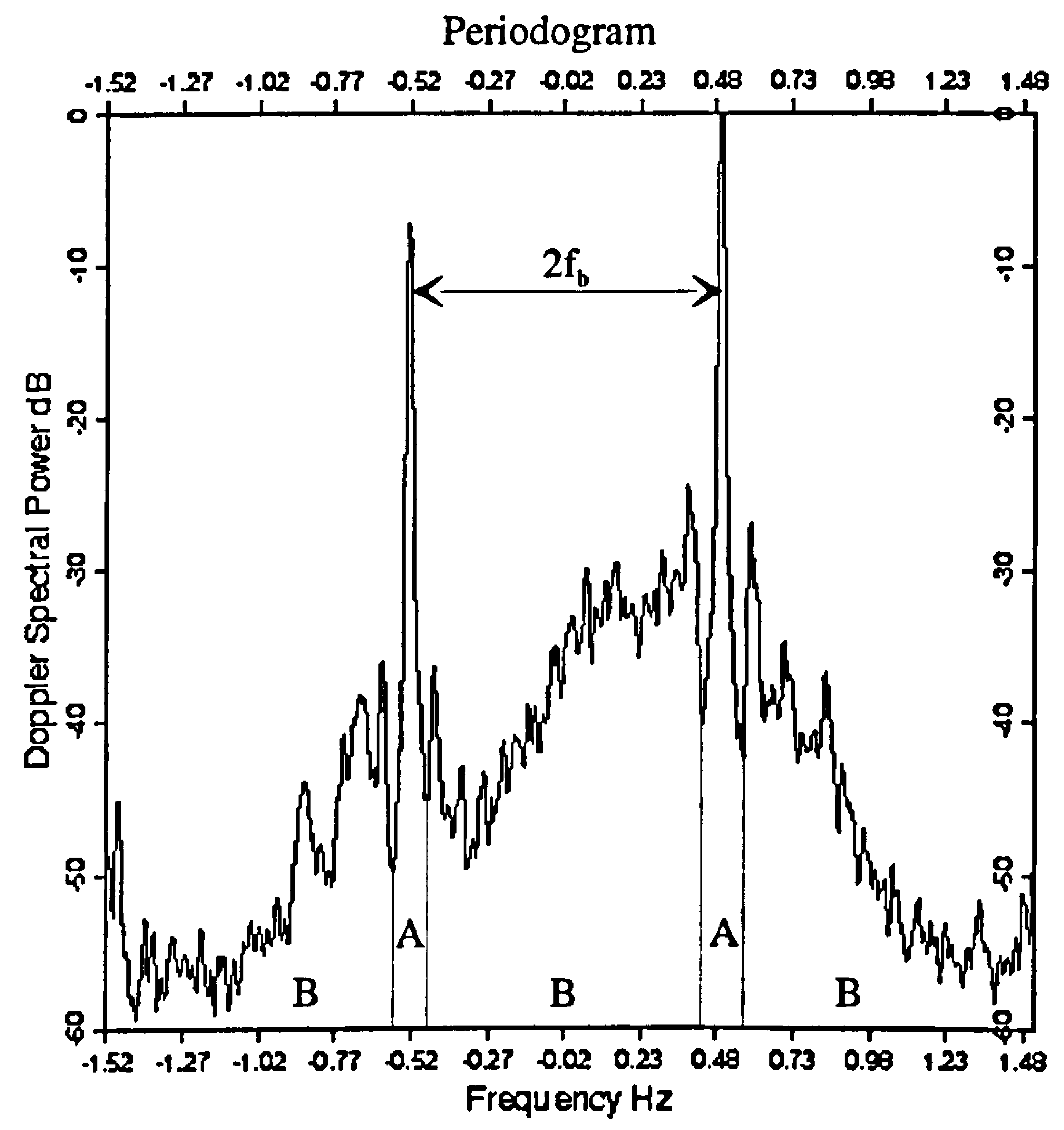

Figure 2.4: Doppler spectrum from the OSCR system showing (A) first- and (B) second-order contributions (measurement taken at 3pm on 27 December 1995). 


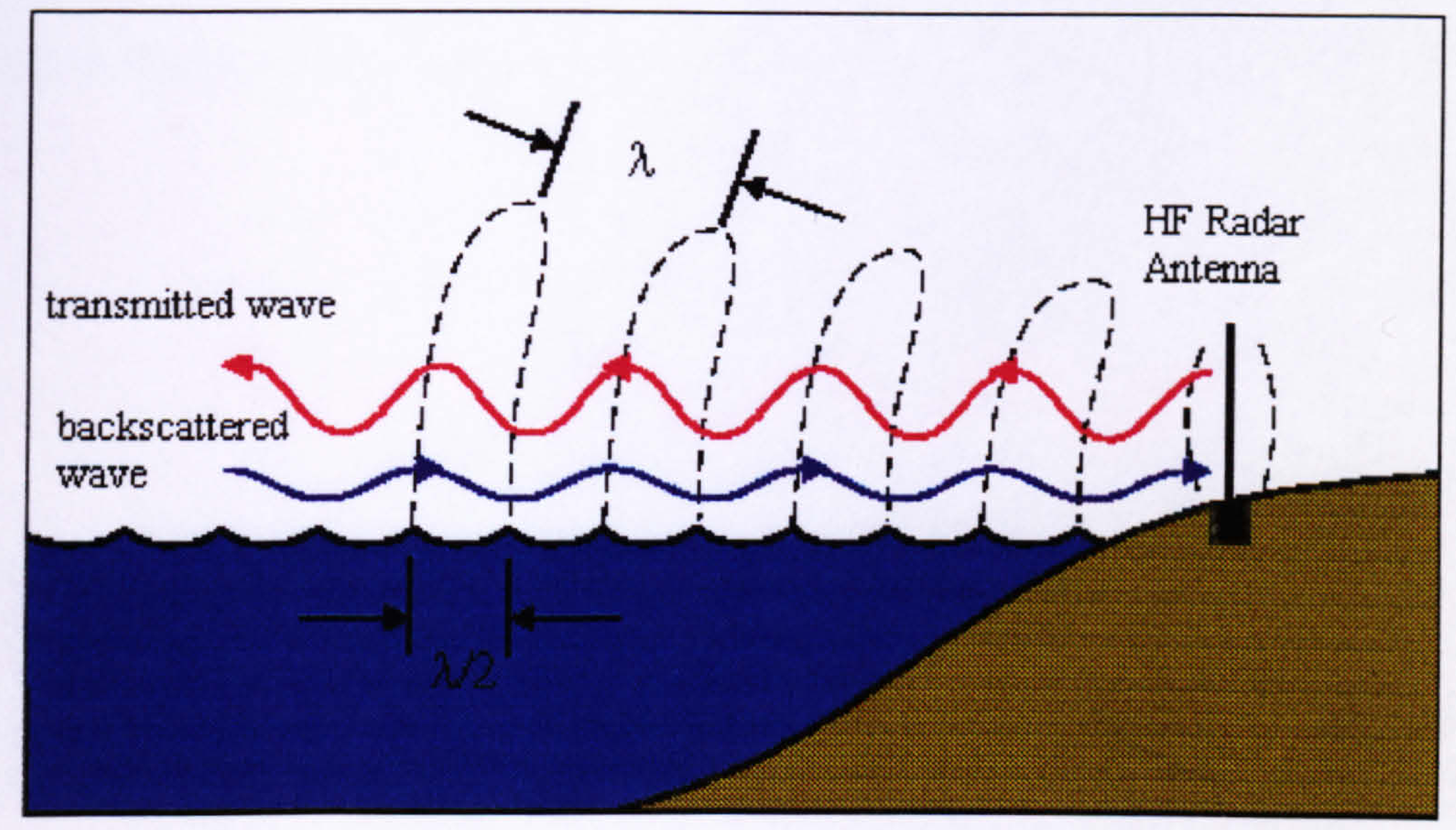

Figure 2.5: Diagram showing the Bragg scatter mechanism, given by the University of Hamburg.

The two Bragg peaks are separated with the expected quantity, $2 f_{b}$, which allows us to check that any spectral method we apply correctly locates the first-order peaks (see Chapter 3). This quantity will be referred to as the theoretical frequency difference given by:

$$
\Delta f_{t}=2 f_{b}=\frac{2}{2 \pi} \sqrt{\frac{4 \pi g f_{\text {radar }}}{c}} .
$$

The difference in amplitude of the two peaks is related to the wind direction, $\theta_{\omega}$. For example, a wind blowing predominantly towards the radar results in the approaching Bragg line that is greater than the receding line. The directional distribution of ocean waves is usually assumed to be of the form:

$$
\cos ^{2 s}\left(\frac{\theta-\theta_{\omega}}{2}\right),
$$

where $s$ is a spreading coefficient.

This model allows sea waves to be generated in all directions except directly upwind, which qualitatively agreed with observations (Heron et al., 1985). Figure 2.6 shows 
different directional distributions from equation (2.12) with $\theta_{\omega}=0^{\circ}$ depending on the choice of the spreading coefficient $s(s=1,2$ and 3).

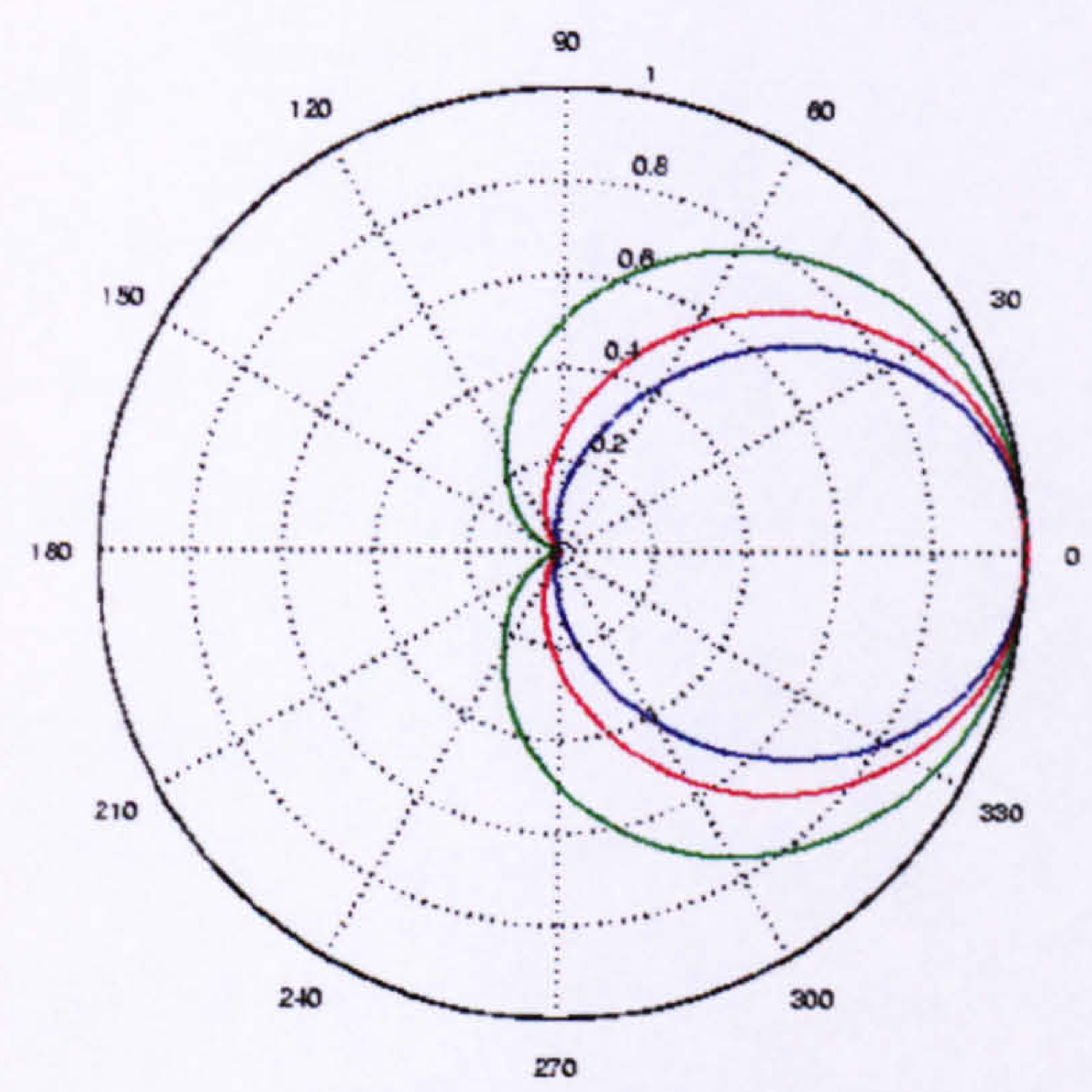

Figure 2.6: Superposition of four directional distributions, corresponding to different spreading coefficients $s$, represented in green for $s=1$, in red for $s=2$ and blue for $s=3$. In this case, the wind direction is $\theta_{\omega}=0^{\circ}$.

The Bragg ratio, $r$, is defined by (Wyatt et al., 1997a)

$$
r=\frac{\sigma^{-}}{\sigma^{+}}=\frac{\cos ^{2 s}\left(\frac{\theta^{-}-\theta_{\omega}}{2}\right)}{\cos ^{2 s}\left(\frac{\theta^{+}-\theta_{\omega}}{2}\right)}
$$

where $\sigma^{+}$and $\sigma^{-}$are the integrals under the positive Bragg peak and negative peak respectively and $\theta^{+}$and $\theta^{-}$are the known propagation directions of the Bragg waves, along the radar beam towards and away from the radar respectively.

If we refer these directions to the radar beam direction, $\theta^{-}=0$ and $\theta^{+}=\pi$, then the equation (2.13) becomes 


$$
r=\frac{\cos ^{2 s}\left(\frac{\theta_{\omega}}{2}\right)}{\sin ^{2 s}\left(\frac{\theta_{\omega}}{2}\right)} \Rightarrow \cos ^{2 s}\left(\frac{\theta_{\omega}}{2}\right)=r \sin ^{2 s}\left(\frac{\theta_{\omega}}{2}\right)
$$

Because $\forall \alpha \in \Re,\left\{\begin{array}{l}\cos ^{2} \alpha=\frac{1+\cos 2 \alpha}{2} \\ \sin ^{2} \alpha=\frac{1-\cos 2 \alpha}{2}\end{array}\right.$, we finally get

$$
\left(1+\cos \theta_{\omega}\right)^{s}=r\left(1-\cos \theta_{\omega}\right)^{s} \Rightarrow \cos \theta_{\omega}=\frac{\sqrt[s]{r}-1}{\sqrt[s]{r}+1}
$$

Figure 2.7 shows the superposition of the different solutions to the equation (2.15) obtained for the wind direction $\theta_{\omega}$ related to the Bragg ratio $r$. In order to have a broad directional distribution, we disregard the solutions given by $s \geq 4$ (see figure 2.7). For example, when $r=2$, we obtain in function of the spreading coefficient $s$ the following angles for $\theta_{\omega}$ :

- $s=1, \theta_{\omega}=70.5^{\circ}$

- $s=2, \theta_{\omega}=80.2^{\circ}$

- $s=3, \theta_{\dot{\omega}}=83.1^{\circ}$

- $s=4, \theta_{\omega}=84.8^{\circ}$

The angular difference obtained with a spreading coefficient $s=1$ and the other spreading coefficients is too large in regard of the error range given by earlier experiments which is around $10^{\circ}$ (Wyatt and Ledgard, 1996). Also, the directional distribution in this case when $s=1$ is too large, in another words, two close values of $r$ result in a large difference between the respective wind directions. Thus, we disregard the solution with a spreading coefficient of 1 . Finally, the spreading coefficient is taken to be two in our case because a broader directional distribution is shown especially when $r \geq 1$ (Wyatt et al., 1997a).

When the radar beam direction, $\theta_{R}$, is taken into account, we then obtain

$$
\cos \left(\theta_{\omega}-\theta_{R}\right)=\frac{\sqrt{r}-1}{\sqrt{r}+1}
$$


With a single radar measurement, the obtained value of $\theta_{\omega}$ can be either clockwise or anticlockwise from the radar beam; to resolve this ambiguity a second radar is used.

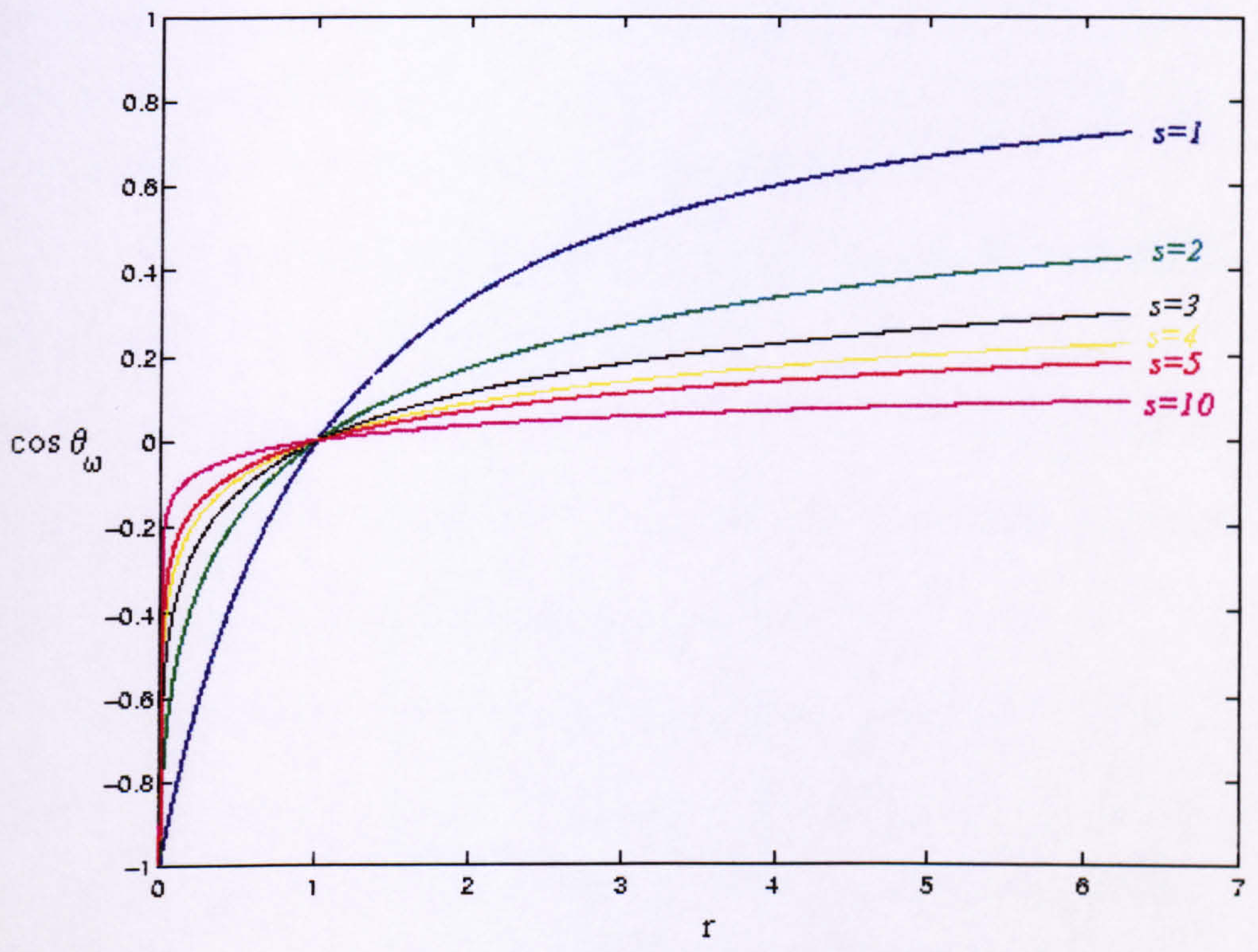

Figure 2.7: Superposition of different curves describing the relationship between the cosine of the wind direction $\theta_{\omega}$ and the Bragg ratio $r$.

\subsection{Second-order measurements}

In contrast to the "spiky" feature of the first-order effect, the second-order continuum surrounding the main Bragg peaks, marked as B in figure 2.4, is a continuous function of frequency. It is produced by: 1. non-linear ocean waves of the correct wavelength which do not satisfy the linear dispersion relationship propagating with different speeds and giving rise to different Doppler shifts; and 2. double electromagnetic scattering processes also giving different Doppler shifts. From hydrodynamic and electromagnetic theory, Barrick (1977) developed an expression for the second-order 
spectrum. He showed that the second-order part of the backscattered spectrum may be described by the following normalised equation :

$$
\sigma_{2}(\eta)=\int_{\theta_{1}}^{\theta_{2}} K(\eta, \theta) S\left(k^{\prime}, \theta^{\prime}\right) S(k, \theta) d \theta
$$

where $K$ is a coupling function describing double scattering processes and non-linear interactions between pairs of ocean waves, which define its two constituent parts: the electromagnetic and hydrodynamic coupling coefficients,

$\eta$ is the normalised Doppler frequency (such that the negative Bragg peak occurs at $\eta=-1$ and the positive Bragg peak at $\eta=+1$ ),

$k=k(\eta, \theta)$ and $k^{\prime}=k^{\prime}\left(\eta^{\prime}, \theta^{\prime}\right)$ are the ocean wavenumbers of the interacting waves obeying the constraint $\vec{k}+\vec{k}^{\prime}=2 \vec{k}_{0}$ where $k_{0}$ is the radar wavenumber,

$S(k, \theta)$ is the full directional wave spectrum,

and $\theta_{1}$ and $\theta_{2}$ define limiting angles (Holden and Wyatt, 1992).

$\sigma_{2}(\eta)$ is the normalised power spectrum of the second-order backscatter. It is divided by the first-order Bragg power in order to cancel unknown factors such as path loss and system gains. This convenient normalization was first suggested by Hasselmann (1971). The full directional spectrum, $S(k, \theta)$, can be expressed by (Tucker 1991):

$$
S(k, \theta)=S(f) G(f, \theta)
$$

where $S(f)$ is one-dimensional spectrum and $G(f, \theta)$ expresses how the energy at frequency $f$ is distributed by direction of wave travel.

The aim of the work at Sheffield is to assess the use of HF radar systems for measuring $S(k, \theta)$. Parameters such as significant waveheight, mean period and mean direction can be determined from $S(k, \theta)$.

The significant waveheight, $H_{S}$, is defined as (Tucker, 1991)

$$
H_{S}=4 \sqrt{\int_{0}^{\infty} \phi(f) d f}
$$


where $\frac{d f}{d k} \phi(f)=\int_{0}^{2 \pi} S(k, \theta) d \theta$ and $\phi(f)$ is the spectral density function. In other words, $H_{S}$ provides a measure of the total energy in the wave spectrum.

The mean wave period $T_{\omega}$ is related to the inverse of the average frequency of the spectrum and is defined as

$$
T_{\omega}=\frac{\int \phi(f) d f}{\int \phi(f) f d f}
$$

By inverting the non-linear Fredholm equation (2.17), the full directional wave spectrum, $S(k, \theta)$, can be determined. A number of methods have been developed to solve this equation (Lipa et al., 1986 and Howell, 1990). For this work, we use the Wyatt inversion method (Wyatt, 1990b and Wyatt and Atanga, 1997b). Initial conditions concerning the wave parameters (such as $H_{S}, T_{\omega}$ and shortwave direction) are necessary to initiate this inversion method. They are extracted directly from the second-order continuum when normalized with respect to the first-order spectrum. These first estimates are used to model the wind wave spectrum, $S\left(k^{\prime}, \theta^{\prime}\right)$ in equation (2.17), as a Pierson-Moskowitz spectrum with a $\cos ^{4}$ directional distribution (Wyatt and Atanga, 1997b).

We are interested in these expressions for $H_{S}$ and $T_{\omega}$ because they will be useful to make comparisons between the periodogram and the selected modern spectral method (see Chapter 3). If we can measure these parameters with sufficient accuracy from our new spectral estimates we can proceed to test the accuracy of the inversion.

\subsubsection{Significant waveheight, $H_{s}$}

Two expressions for $H_{s}$ using $\sigma_{2}(\eta)$, have been developed by Wyatt (1986):

- for the waves traveling perpendicular to the radar beam:

$$
H_{s}=\alpha R, \alpha \in \Re^{+} \text {regression coefficient }
$$

- for all other waves: 


$$
H_{s}=\beta R^{\gamma}, \beta, \gamma \in \Re^{+} \text {regression coefficients }
$$

where $R=\sqrt{\int \sigma_{F_{L}} \sigma_{2}(\eta) d \eta}+\sqrt{\int \sigma_{2}(\eta) d \eta}, \eta$ being the normalised Doppler frequency (such that $\eta=-1$ at the negative Bragg peak and $\eta=1$ at the positive one),

$\sigma_{2}(\eta)$ the normalised power spectrum of the second-order backscatter (it is divided by the first-order Bragg power),

$$
F_{L}=\left[0.4 \eta_{B}, \eta_{B}\right] \backslash\left\{\eta \in F_{B}\right\}, F_{U}=\left[\eta_{B}, 1.6 \eta_{B}\right] \backslash\left\{\eta \in F_{B}\right\},
$$

$F_{B}=\{$ frequencies $\eta$ contributing to the first - order spectrum $\}$,

and $\eta_{B}=1$, the normalised Bragg frequency.

A question arises: which of these two values for $H_{S}$ is the correct one? This can be only determined with additional information from a second radar or another system, such as wave buoy system (see Chapter 5 section 5.2.1). When two HF radar systems are used, the final value for $H_{s}$ is obtained by averaging the two closest quantities given by equations (2.21) or (2.22) resulting from each radar.

\subsubsection{Mean wave period, $T_{\omega}$}

As for $H_{S}$, a formula for $T_{\omega}$ has been derived (Wyatt, 1986):

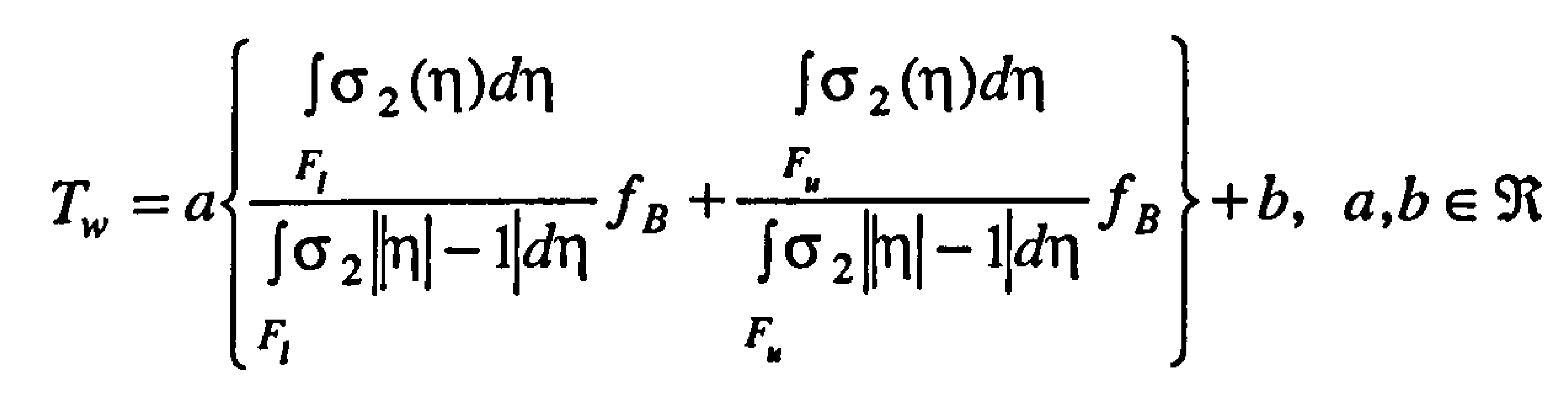

where $f_{B}$ is the Bragg frequency,

$$
F_{l}=\left[0, \eta_{B}\right] \backslash\left\{\eta \in F_{B}\right\}, F_{u}=\left[\eta_{B}, 2 \eta_{B}\right] \backslash\left\{\eta \in F_{B}\right\},
$$

and $a$ and $b$ are regression coefficients. 


\subsection{Data analysis}

In the following chapters, we will use the fact that the data are complex Gaussian. So we need to check this property for HF radar data.

The stationarity of the data was assumed for the application of the periodogram in section 2.3.1 and this property has always been assumed for HF radar data. Since to show the stationarity of a signal is a very difficult task, this assumption is also made in this work. This will be necessary for the application of modern spectral techniques presented in the next chapter and the application of the Monte Carlo simulation study, a method of comparison described in Chapter 5. But, we propose here a preliminary investigation using the Wavelet analysis, a time-frequency method, which allows us to analyse non-stationary and interference effects present in the data. For some cases of HF radar data, non-stationarity has been identified with the Wavelet analysis.

\subsubsection{Complex normality}

Complex normality stands for a complex process following a Gaussian distribution. To investigate complex normality, we show first the normality of the real and imaginary parts of the data and then, the zero-correlation between the real and imaginary parts.

\subsubsection{Normality}

We have used an algorithm from Numerical Recipies in C (Press et al., 1988): the Kolmogorov-Smirnov test. The program is included in Appendix $\mathbf{C}$ because there are errors in the published code (p492). The test is well explained by L. Sachs (1984, p 330).

The Kolmogorov-Smirnov goodness of fit test is:

$$
\begin{aligned}
& H_{0}: F(x)=F_{0}(x) \text { for all } \mathrm{x} \\
& \neq H_{A}: F(x) \neq F_{0}(x) \text { for some } \mathrm{x}
\end{aligned}
$$


the null hypothesis, $H_{0}$, that the sample comes from a population with known distribution function, $F_{0}(x)$, is tested against the alternate hypothesis, $H_{A}$, that the population underlying the sample does not have $F_{0}(x)$ as its distribution.

The Kolmogorov-Smirnov statistic measures discrepancy as the maximum absolute difference:

$$
D=\max _{x}\left|F_{N}(x)-F_{0}(x)\right|
$$

where $F_{N}(x)=\frac{1}{N} \times($ number of observations $\leq x)$ and $N$ the number of data points.

The significance level, $\alpha_{K S}$, of an observed value of $D$ is given by the probability

$$
P(D>\text { observed })=Q_{K S}(\sqrt{N} D)=\alpha_{K S}
$$

where $Q_{K S}(\lambda)=2 \sum_{j=1}^{\infty}(-1)^{j-1} e^{-2 j^{2} \lambda^{2}}$ is a monotonic function (Press et al., 1988, p491) satisfying the limit conditions :

$$
\left\{\begin{array}{l}
Q_{K S}(0)=1 \\
Q_{K S}(\infty)=0
\end{array} .\right.
$$

Large values of $D(D \geq 0.05)$ show that the cumulative distribution function of data is significantly different from the normal distribution.

We did several Kolmogorov-Smirnov tests for each system (PISCES and OSCR) on the real part $\left\{x_{1}, \ldots, x_{N}\right\}$ and the imaginary part $\left\{y_{1}, \ldots, y_{N}\right\}$ of the data with $N=1000$, and small values of $D$ were obtained. For example, with the PISCES and OSCR files used to produce the periodograms seen in figures 2.2 and 2.3, we found:

- OSCR file : $\left\{\begin{array}{c}D=0.027 \text { (real part) } \\ D=0.017 \text { (imaginary part) }\end{array}\right.$

- PISCES file : $\left\{\begin{array}{c}D=0.027 \text { (real part) } \\ D=0.026 \text { (imaginary part) }\end{array}\right.$

Therefore, we can accept the normality for both real and imaginary parts of the HF radar data. 


\subsubsection{Correlation}

We use the Pearson's correlation coefficient (Press et al., 1988):

$$
r_{\text {Pearson }}=\frac{\sum_{i}\left(x_{i}-\bar{x}\right)\left(y_{i}-\bar{y}\right)}{\sqrt{\sum_{i}\left(x_{i}-\bar{x}\right)^{2}} \sqrt{\sum_{i}\left(y_{i}-\bar{y}\right)^{2}}}
$$

for pairs of quantities $\left(x_{i}, y_{i}\right)_{i=1 . N}$, where $\bar{x}$ and $\bar{y}$ are respectively the mean of the $x_{i}$ 's and $y_{i}$ 's.

In our case, the pairs $\left(x_{i}, y_{i}\right)_{i=1 . N}$ represent respectively the real and imaginary parts of the data.

To have no correlation, we must have $\left|r_{\text {Pearson }}\right|<0.05$, it must be close to 0 . If $\left|r_{\text {Pearson }}\right|=1$, then it means complete correlation.

For both systems, different files were tested and correlation was not present between the real and imaginary parts. For example, with the data used to produce the periodograms seen in figures 2.2 and 2.3 , we find that $\left|r_{\text {Pearson }}\right|=0.001$.

Therefore, since the zero-correlation between the real and imaginary parts of the data can be assumed and since the normality of the different parts were shown, we can assume that the data are complex Gaussian.

\subsubsection{Wavelet analysis}

This section introduces the wavelet analysis which describes how the spectral content of a signal changes in time. This time-frequency analysis provides the opportunity to interpret interference and non-stationary effects and show the quality of the data.

Until now, we have used the Doppler spectrum with its two distinct peaks in order to get wave parameters (e.g. significant wave height, mean period, etc.), but we do not know when the frequencies of these two peaks occurred.

In Chapter 1, we saw that the time variations of the Bragg frequencies are related to the ocean wave height (Kahn 1991), in other words to the second-order contribution. Further information in the time domain might help to understand these variations and 
then interpret interference effects. Therefore, we are interested in applying the wavelet analysis to HF radar data.

The Wavelet Transform (WT) is of interest for the analysis of non-stationary signals, because it provides an alternative to the classical Short-Time Fourier Transform (STFT).

In contrast to the STFT, which uses a single analysis window, the WT uses short windows at high frequencies and long windows at low frequencies.

For some applications it is desirable to see the WT as a signal decomposition onto a set of basis functions, called wavelets. They are obtained from a single prototype wavelet by dilations and contractions. The prototype wavelet can be thought of as a bandpass filter. In a WT, the notion of scale is introduced as an alternative to frequency, leading to a so-called time-scale representation. This means that a signal is mapped into a time-scale plane (the equivalent of the time-frequency plane used in the STFT).

\subsubsection{Non-Stationary Signal Analysis}

The aim of signal analysis is to extract relevant information from a signal by transforming it.

For stationary signals $x(t)$, that is, signals whose statistical properties do not involve time, the natural "stationary transform" is the well-known Fourier transform:

$$
X(f)=\int_{-\infty}^{+\infty} x(t) e^{-2 j \pi f t} d t
$$

The analysis coefficients $X(f)$ define the notion of global frequency, $f$, in a signal. They are computed as inner products of the signal with sinewave basis functions of infinite duration. As a result, Fourier analysis works well if $x(t)$ is composed of a few stationary components.

However, any abrupt change in time in a non-stationary signal $x(t)$ is spread out over the whole frequency axis in $X(f)$.

Therefore, an analysis adapted to non-stationary signals requires more than the Fourier Transform. The usual approach is to introduce time dependency in the Fourier 
analysis while preserving linearity. The idea is to introduce a "local frequency" parameter (local in time) so that the "local" Fourier Transform looks at the signal through a window over which the signal is approximately stationary. Another (equivalent) way is to modify the sinewave basis functions used in the Fourier Transform to basis functions which are more concentrated in time (but less concentrated in frequency).

\subsubsection{The Short-Time Fourier Transform : Analysis with Fixed Resolution}

Consider a signal $x(t)$ and assume it is stationary when seen through a window $g(t)$ of limited extent, centered at time location $\tau$.

The FT of the windowed signals $x(t) g^{*}(t-\tau)$ yields the STFT:

$$
\operatorname{STFT}(\tau, f)=\int x(t) g^{*}(t-\tau) e^{-2 j \pi f t} d t
$$

which maps the signal into a two-dimensional function in a time-frequency plane $(\tau, f)$.

The parameter $f$ is similar to the Fourier frequency and many properties of the Fourier transform carry over to the STFT. However, the analysis here depends critically on the choice of the window $g(t)$.

An alternative view is based on a filter bank interpretation of the same process. At a given frequency $f$, equation (2.28) amounts to filtering the signal " at all times " with a bandpass filter having as impulse response the window function modulated to that frequency.

A possible drawback to the time and frequency resolution can be shown. Consider the ability of the STFT to discriminate between two pure sinusoids. Given a window function $g(t)$ and its Fourier transform $G(f)$, define the bandwidth $\Delta f$ of the filter

as

$$
\Delta f^{2}=\frac{\int f^{2}|G(f)|^{2} d f}{\int|G(f)|^{2} d f}
$$

where the denominator is the energy of $g(t)$. 
Two sinusoids will be discriminated only if they are more than $\Delta f$ apart. Thus, the resolution in frequency of the STFT analysis is given by $\Delta f$. Similarly, the spread in time is given by $\Delta t$ as :

$$
\Delta t^{2}=\frac{\int t^{2}|g(t)|^{2} d t}{\int|g(t)|^{2} d t}
$$

where the denominator is again the energy of $g(t)$.

Two pulses in time can be discriminated only if they are more than $\Delta t$ apart.

Now, resolution in time and frequency cannot be arbitrarily small, because their product is lower bounded.

The Time - Bandwidth product must satisfy the inequality: $\Delta t \Delta f \geq 1 / 4 \pi$ : the Uncertainty principle or Heisenberg inequality. It means that one can only trade time resolution for frequency resolution, or vice versa. Gaussian windows are therefore often used since they meet the bound with equality.

More important is that once a window has been chosen for the STFT, then the timefrequency resolution given by (2.29) and (2.30) is fixed over the entire time-frequency plane since the same window is used at all frequencies.

\subsubsection{The Continuous Wavelet Transform : a multiresolution Analysis}

To overcome the resolution limitation of the STFT, one can imagine letting the resolution $\Delta t$ and $\Delta f$ vary in the time-frequency plane in order to obtain a multiresolution analysis. Intuitively, when the analysis is viewed as a filter bank, the time resolution must increase with the central frequency of the analysis filters. We therefore impose that $\Delta f$ is proportional to $f$ :

$$
\frac{\Delta f}{f}=c \text { where } \mathrm{c} \text { is a constant. }
$$

The analysis filter bank is then composed of band-pass filters with constant relative bandwidth (so-called " constant-Q" analysis). Another way to say this is that, instead of the frequency responses of the analysis filter being regularly spaced over the frequency axis (as for the STFT case), they are regularly spread in a logarithmic scale. 
When (2.31) is satisfied, we see that $\Delta f$ and therefore also $\Delta t$ changes with the centre frequency of the analysis filter. Of course, they still satisfy the Heisenberg inequality (2.30), but now, the time resolution becomes arbitrarily good at high frequencies, while the frequency resolution becomes arbitrarily good at low frequencies.

A generalization of the concept of changing resolution at different frequencies is obtained with so-called “ wavelet packets ", where arbitrary time-frequency resolutions (within the uncertainty bound (2.30) ) are chosen depending on the signal. The Continuous Wavelet Transform (CWT) follows the above ideas while adding a simplification: all impulse responses of the filter bank are defined as scaled, (stretched or compressed) versions of the same prototype $h(t)$ :

$$
h_{a}(t)=\frac{1}{\sqrt{|a|}} h(t / a)
$$

where $a$ is a scale factor. The constant $\frac{1}{\sqrt{|a|}}$ is used for energy normalization. This results in the definition of the CWT:

$$
C W T_{x}(\tau, a)=\frac{1}{\sqrt{|a|}} \int x(t) h^{*}\left(\frac{t-\tau}{a}\right) d t
$$

To make the connection with the modulated window used in the STFT clearer, the basic wavelet $h(t)$ in (2.32) could be chosen as a modulated window:

$$
h(t)=g(t) e^{-2 j \pi f_{0} t}
$$

Then the frequency responses of the analysis filters indeed satisfy (2.31) with the identification:

$$
a=\frac{f_{0}}{f}
$$

The local frequency $f=\frac{f_{0}}{a}$, whose definition depends on the basic wavelet, is no longer linked to frequency modulation (as was the case for the STFT) but is now related to time-scalings. This is the reason why the terminology " scale" is preferred to "frequency" for the CWT, the word " frequency " being reserved for the STFT.

Note that we define scale in wavelet analysis like the scale in geographical maps: since the filter bank impulse responses in (2.32) are dilated as scale increases, large 
scale corresponds to contracted signals, while small scale corresponds to dilated signals.

\subsubsection{Wavelet Analysis and Synthesis}

Another way to introduce the CWT is to define wavelets as basis functions:

$$
(2.31) \Leftrightarrow C W T_{x}(\tau, a)=\int x(t) h_{a, \tau}^{*}(t) d t
$$

which measures the "similarity" between the signal and the basis functions

$$
h_{a, \tau}(t)=\frac{1}{\sqrt{a}} h\left(\frac{t-\tau}{a}\right) \text { called wavelets. }
$$

The wavelets are scaled and translated versions of the basic wavelet prototype $h(t)$.

The reconstruction of $x(t)=c \iint_{a>0} C W T_{x}(\tau, a) h_{a, \tau}(t) \frac{d a d \tau}{a^{2}}$

is satisfied whenever $h(t)$ is of finite energy and band pass (which implies that it oscillates in time like a short wave, hence the name " wavelet "). More precisely, if $h(t)$ is assumed sufficiently regular, then the reconstruction condition is $\int h(t) d t=0$. Note that the reconstruction takes place only in the sense of the signal's energy. A signal may be reconstructed only with zero mean.

\subsubsection{Scalograms}

The spectrogram, defined as the square modulus of the STFT, is a very common tool in signal analysis because it provides a distribution of the energy of the signal in the time-frequency plane.

A similar distribution can be defined in the wavelet case. Since the CWT behaves like an orthonormal basis decomposition, it can be shown that it preserves energy. We have

$$
\iint\left|C W T_{x}(\tau, a)\right|^{2} \frac{d \tau d a}{a^{2}}=E_{x}
$$

where $E_{x}=\int|x(t)|^{2} d t$ is the energy of the signal $x(t)$. 
This leads us to define the wavelet spectrogram or scalogram as the squared modulus of the CWT. It is a distribution of the energy of the signal in the time-scale plane, associated with measure $\frac{d \tau d a}{a^{2}}$, and thus expressed in power per frequency unit, like the spectrogram. However, in contrast to the spectrogram, the energy of the signal is here distributed with different resolutions.

\subsubsection{Application}

We have been using the Morlet wavelet:

$$
h(t)=e^{2 i \pi t} e^{-0.5 t^{2}-e^{-2 \pi^{2}}}
$$

and analysing the scalograms given by this wavelet basis. The Morlet wavelet is a normalized, Gaussian-enveloped complex sinusoid with zero mean. It is only nearly orthogonal but offers satisfactory resolution and stability.

Figure 2.9 exhibits the scalogram of a good PISCES file, EB0319.011 (measurement taken at 11 am on 19 March 1987). As can be seen, it contains 2 main parts which correspond to the 2 main Bragg peaks given by the spectral estimate shown in figure 2.8. Here, the frequency scale is different from the spectral ones: the zero Doppler is shifted to the right by $1.25 \mathrm{~Hz}$. Between these two rows, there is a "small valley" which means that the frequencies between 0.6 and $1.5 \mathrm{~Hz}$ during all the emission of the signal are not powerful enough to show up (for the power scaling here). Figure 2.10 shows the scalogram from $0.7 \mathrm{~Hz}$ to $1.4 \mathrm{~Hz}$. The power is much lower than the one contained in the peaks of figure 2.9 . 


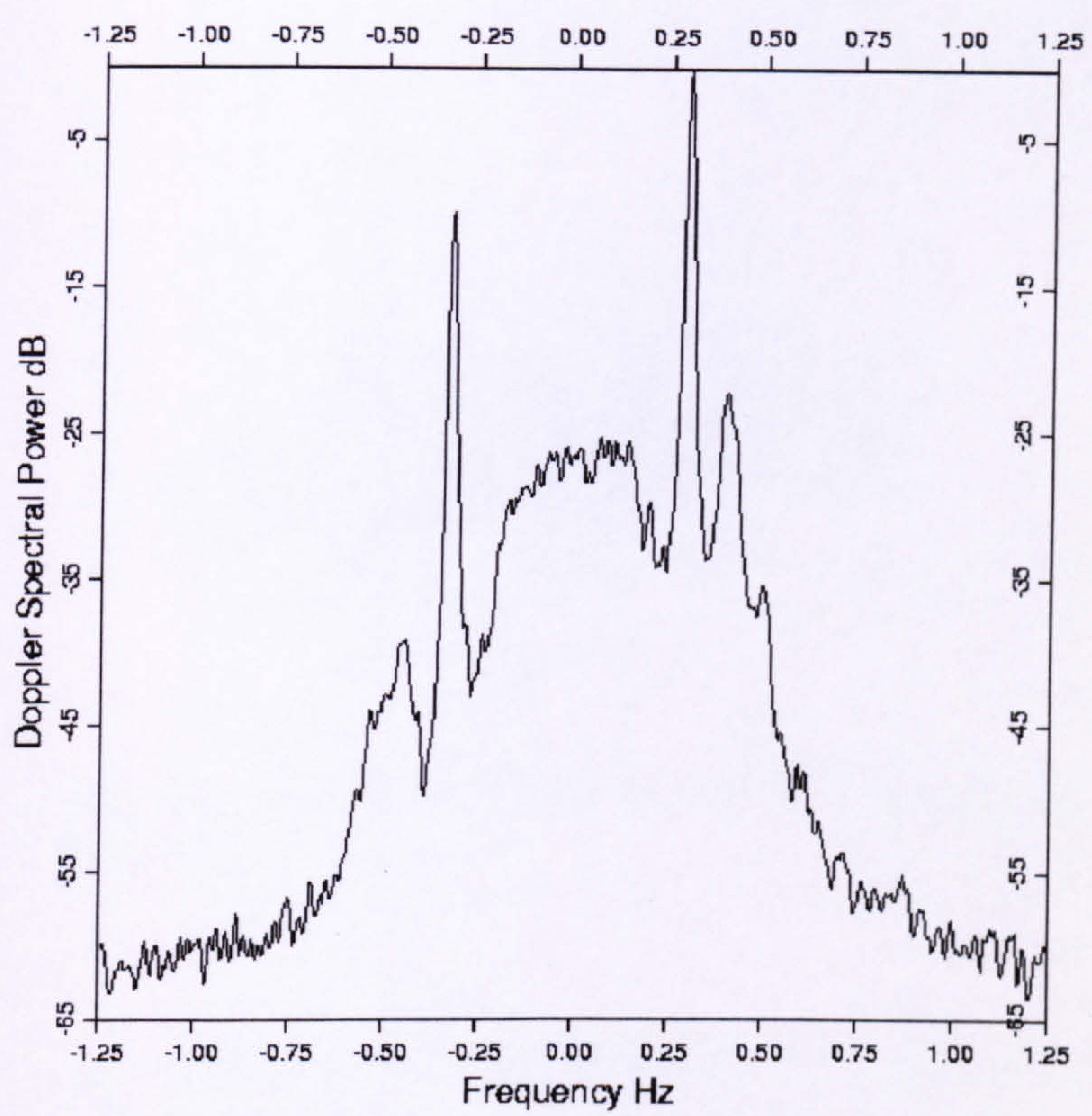

Figure 2.8: Periodogram from the PISCES (measurement taken at 11a.m. on 19 March 1987), $N=4608$ and $P=33$.

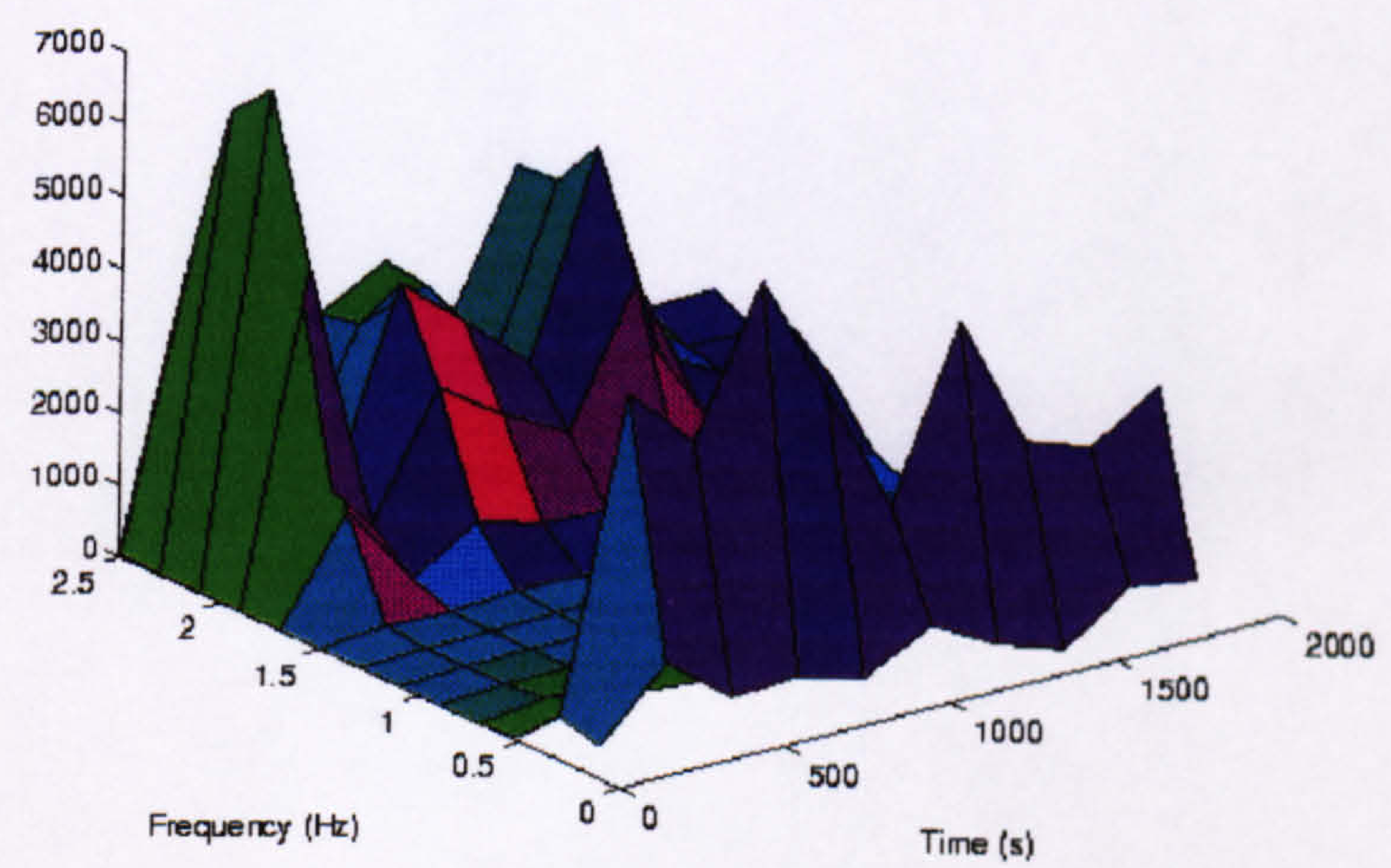

Figure 2.9: Morlet scalogram obtained from the PISCES file EB0319.011. 


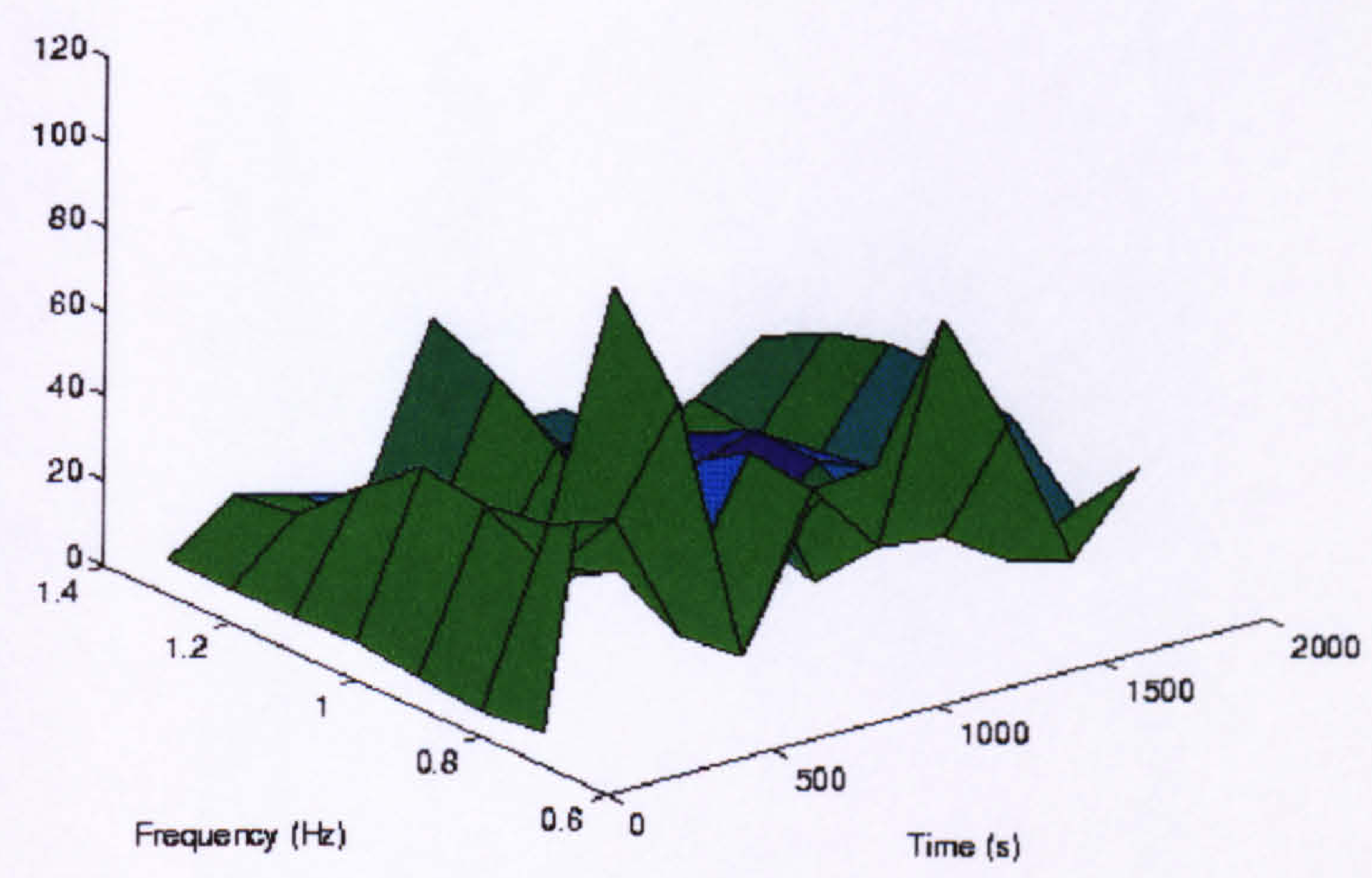

Figure 2.10: Morlet scalogram obtained from the PISCES file EB0319.011, showing the frequencies from $0.7 \mathrm{~Hz}$ to $1.4 \mathrm{~Hz}$.

Plotting the data of this file, we can see that the real and imaginary parts behave in the same way. Figures 2.11 and 2.12 show the first 2000 samples.

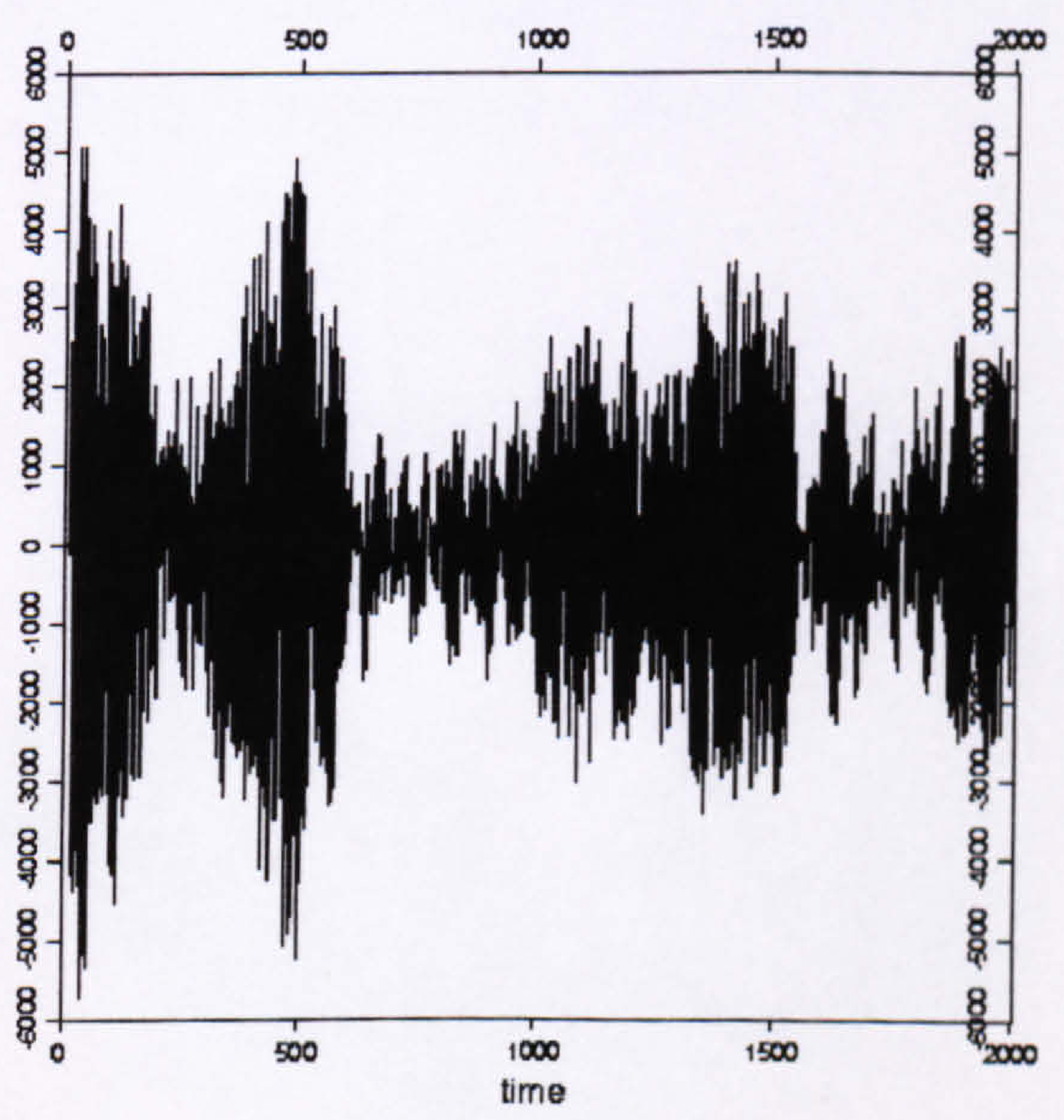

Figure 2.11: First 2000 real data points of the PISCES file EB0319.011. 


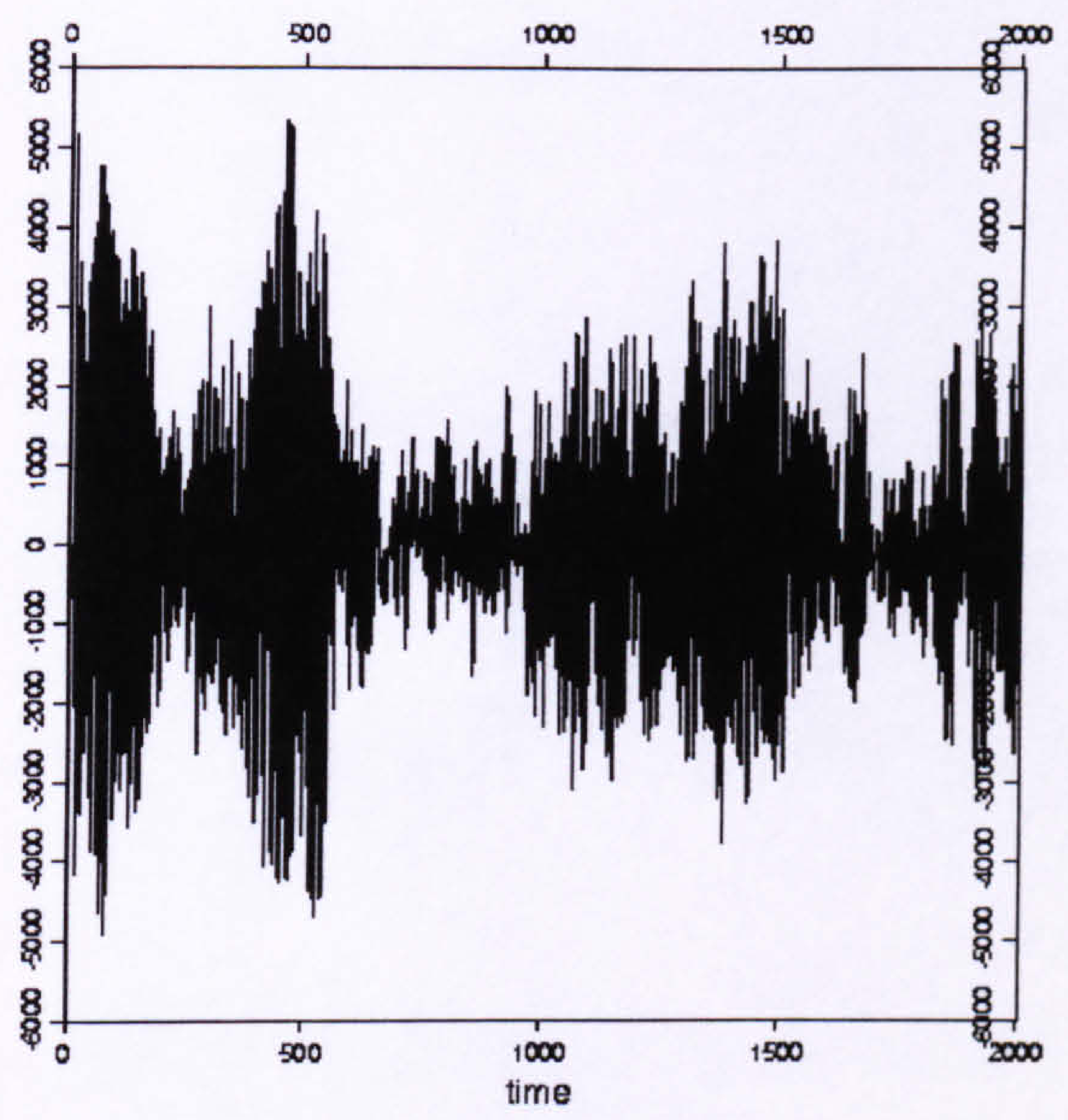

Figure 2.12: First 2000 imaginary data points of the PISCES file EB0319.011.

With reference to the PISCES file EB0316.008 (measurement taken at 8am on 16 March 1987), the scalogram (figure 2.13) seems to be similar when compared with the previous one. Figure 2.14 shows its periodogram. There is some perturbation at the large negative frequencies and the total frequency difference is not very close to the theoretical one: $\Delta f=0.527344 \mathrm{~Hz}$ and $\Delta f_{t}=0.532674 \mathrm{~Hz}$.

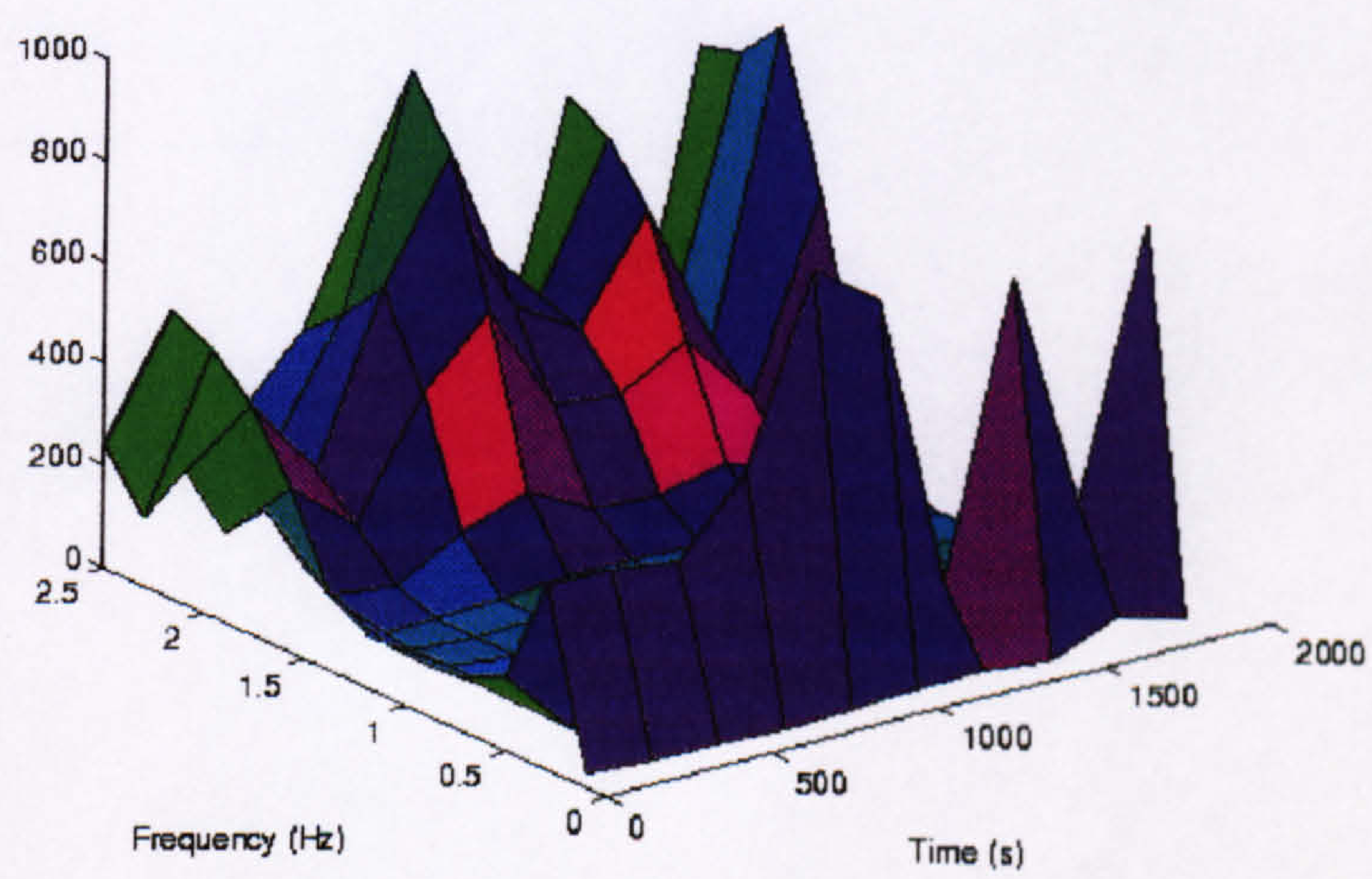

Figure 2.13: Morlet scalogram of the PISCES file EB0316.008. 


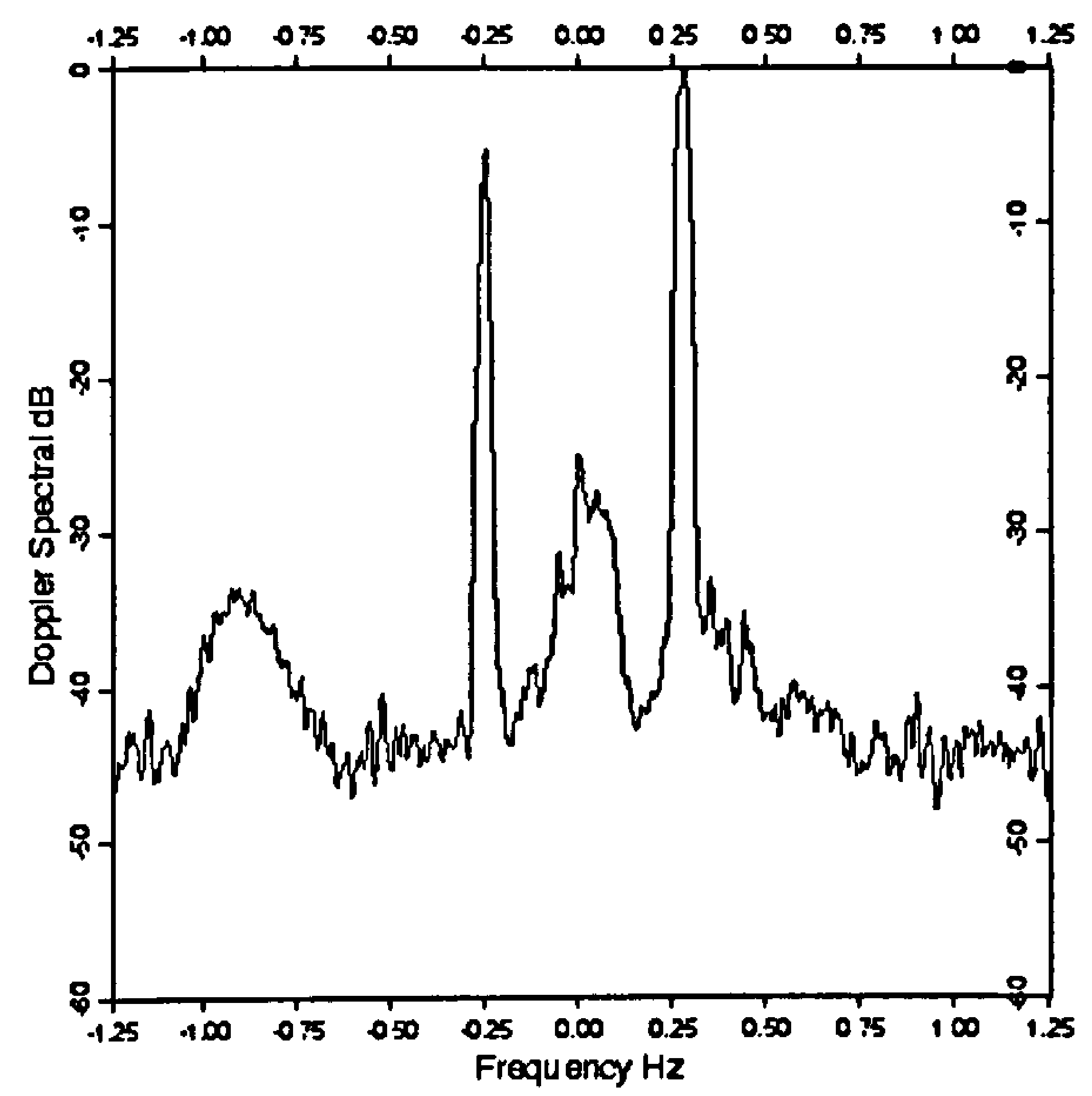

Figure 2.14: Periodogram of the PISCES file EB0316.008.

But if we look more at the scalogram, we can notice that at the beginning (in time) for the first row (foreground), the power tends to have less variability during the 500 seconds (which correspond roughly to the first 1500 data points). Figure 2.15 exhibits the Autoregressive (AR) PSD estimate which does not use the first 2000 samples. This spectral estimate is obtained from a modern spectral method, the modified covariance method, which will be presented in the following chapter and fully described in Chapter 4. It no longer contains the perturbation noted in the figure 2.14 and also a better estimation of $\Delta f$ is obtained: $\Delta f=0.532227 \mathrm{~Hz}$. The signal is plotted in figures $2.16,2.17,2.18$ and 2.19. For the first 2000 data points (figures 2.16 and 2.17$)$, note that the real and imaginary parts are very different particularly at the beginning. For figures 2.17 and 2.18 , they vary in a similar way. 


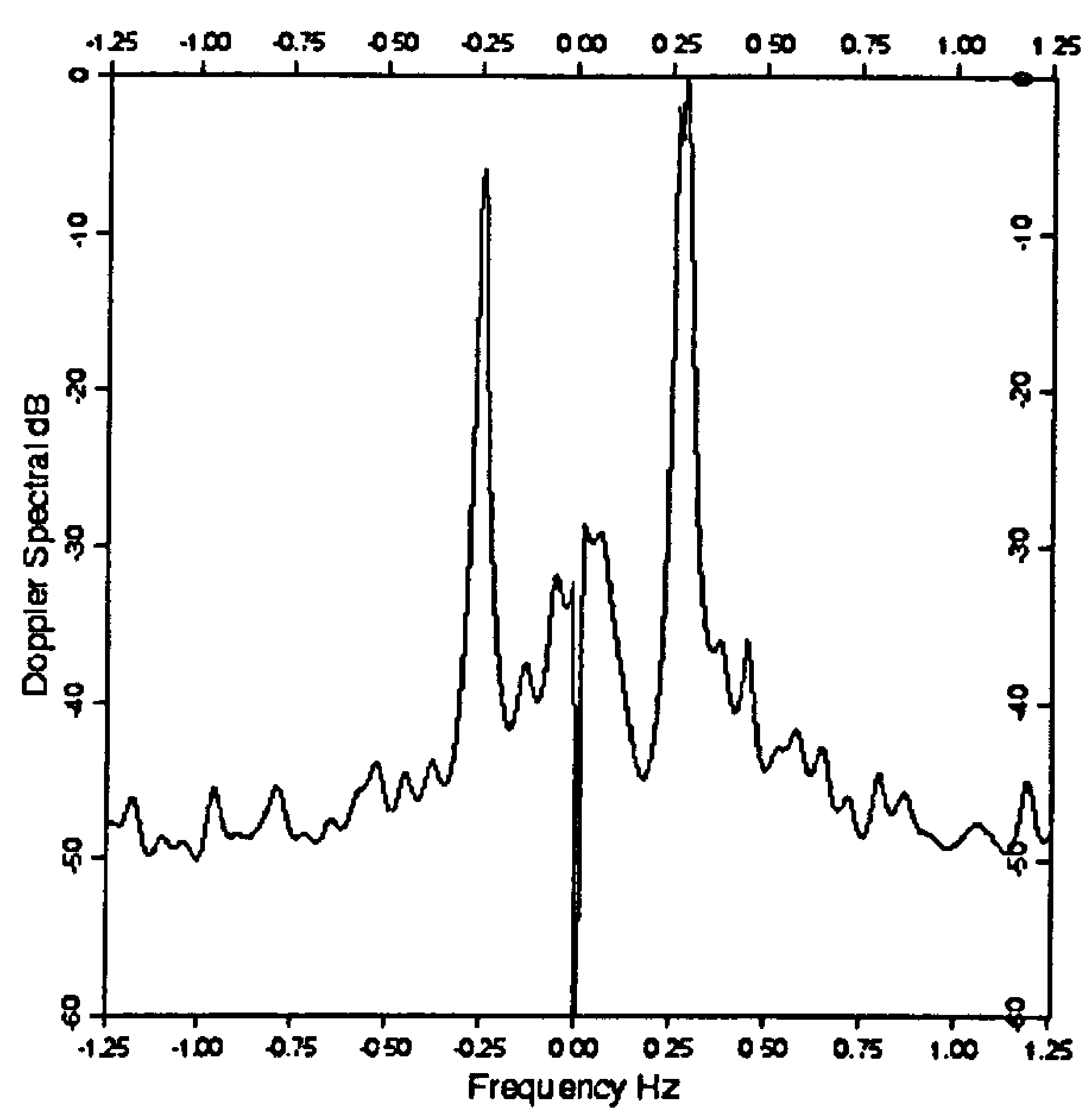

Figure 2.15: AR PSD estimate of the PISCES file EB0316.008. The first 2000 data points are not used and the perturbation of the figure 2.14 has disappeared.

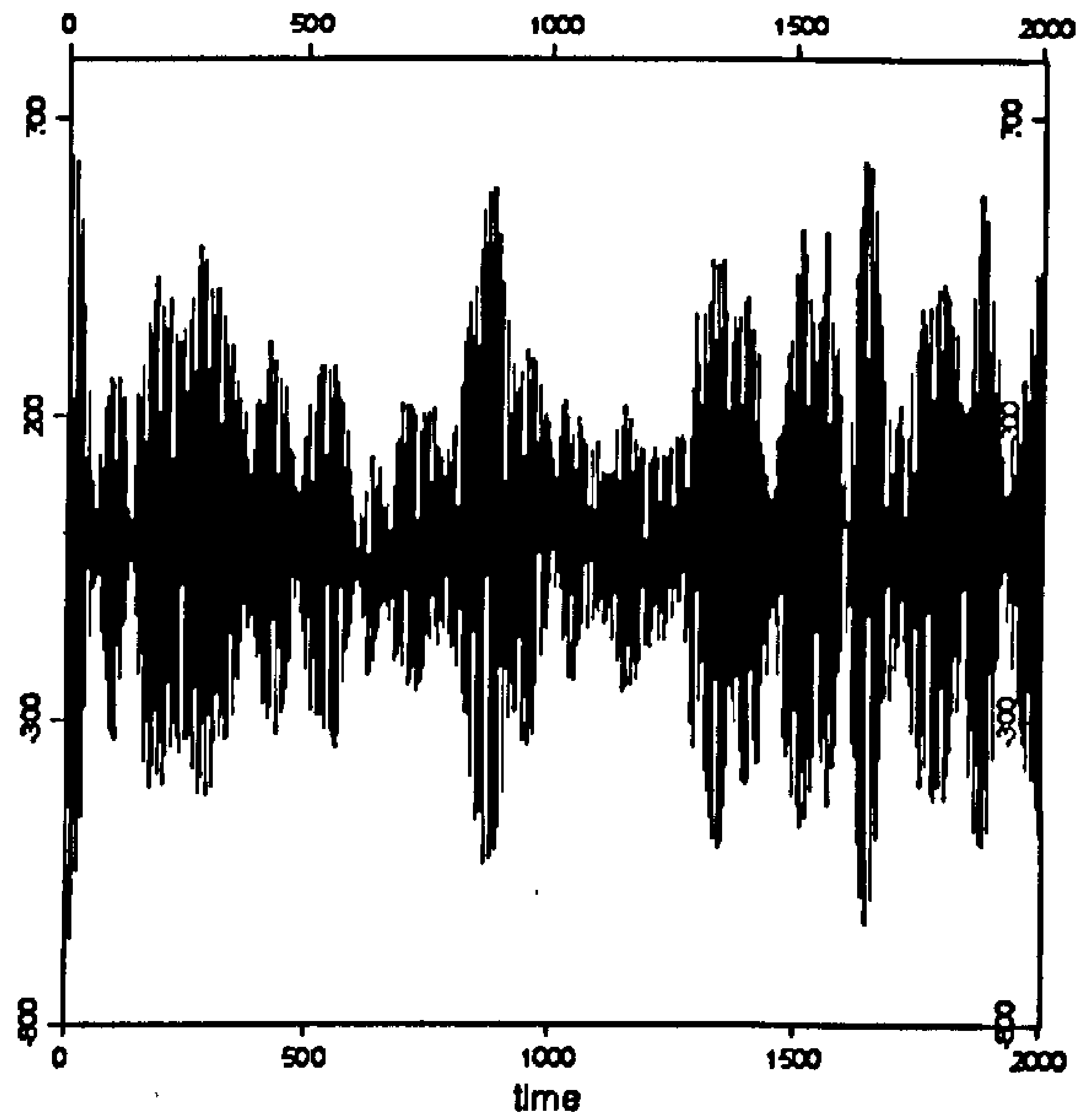

Figure 2.16: Real part of the first 2000 data points of the PISCES file EB0316.008.

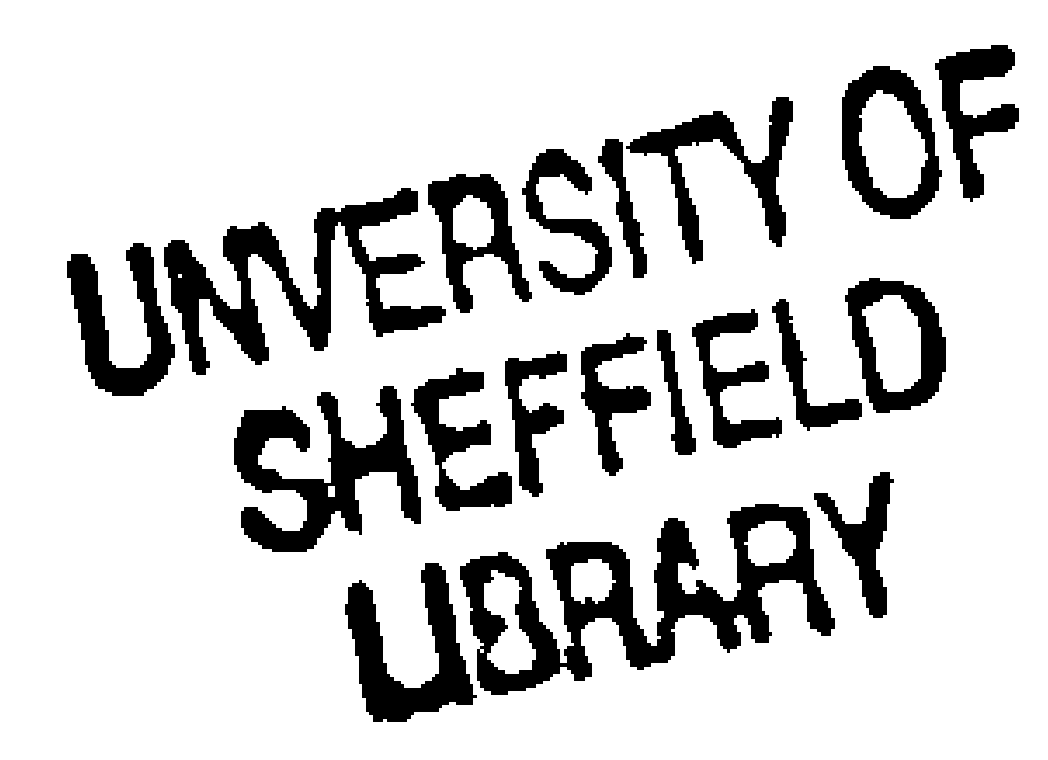




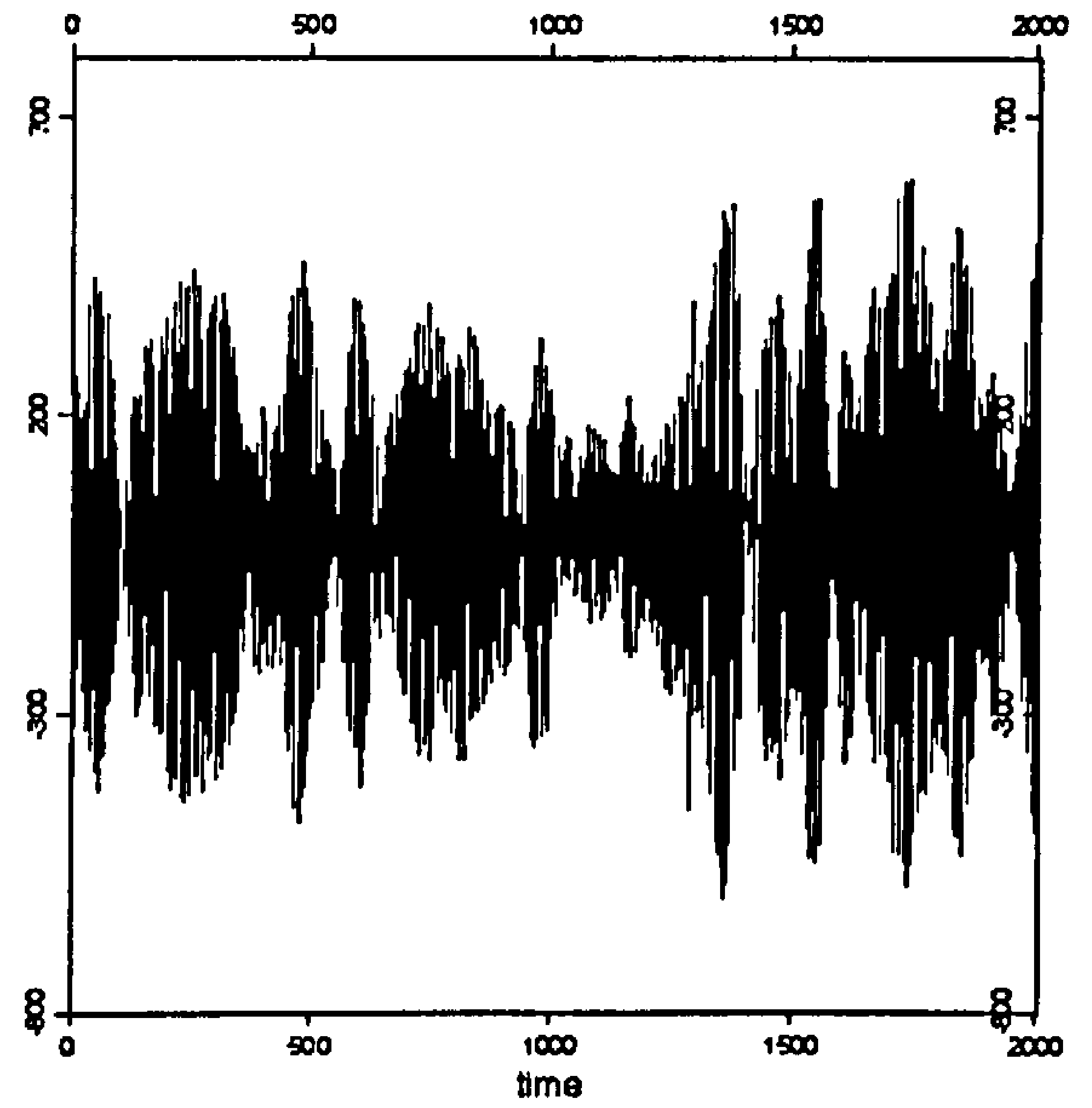

Figure 2.17: Imaginary part of the first 2000 data points of the PISCES file EB0316.008.

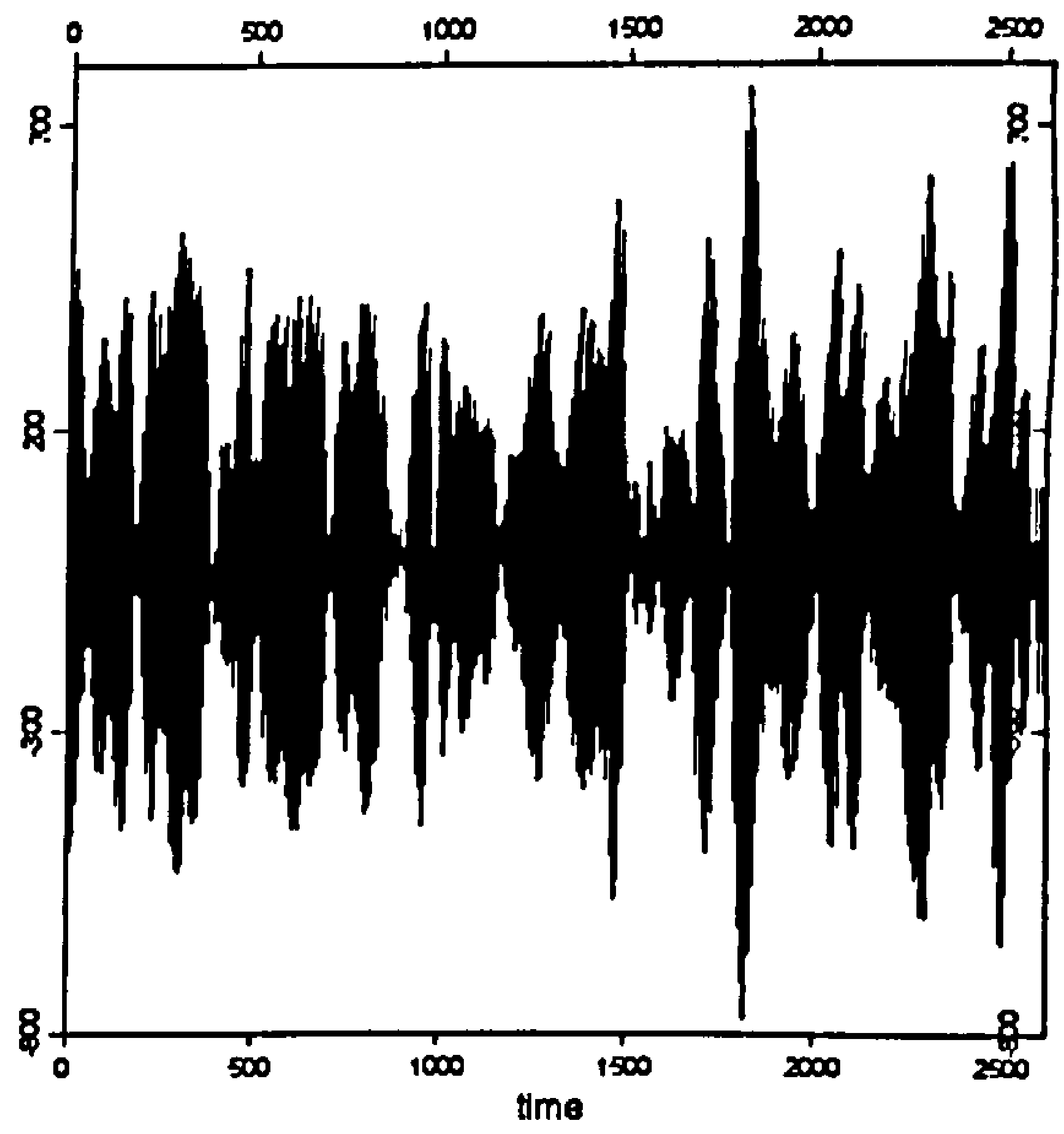

Figure 2.18: Real part of the last 2609 data points of the PISCES file EB0316.008. 


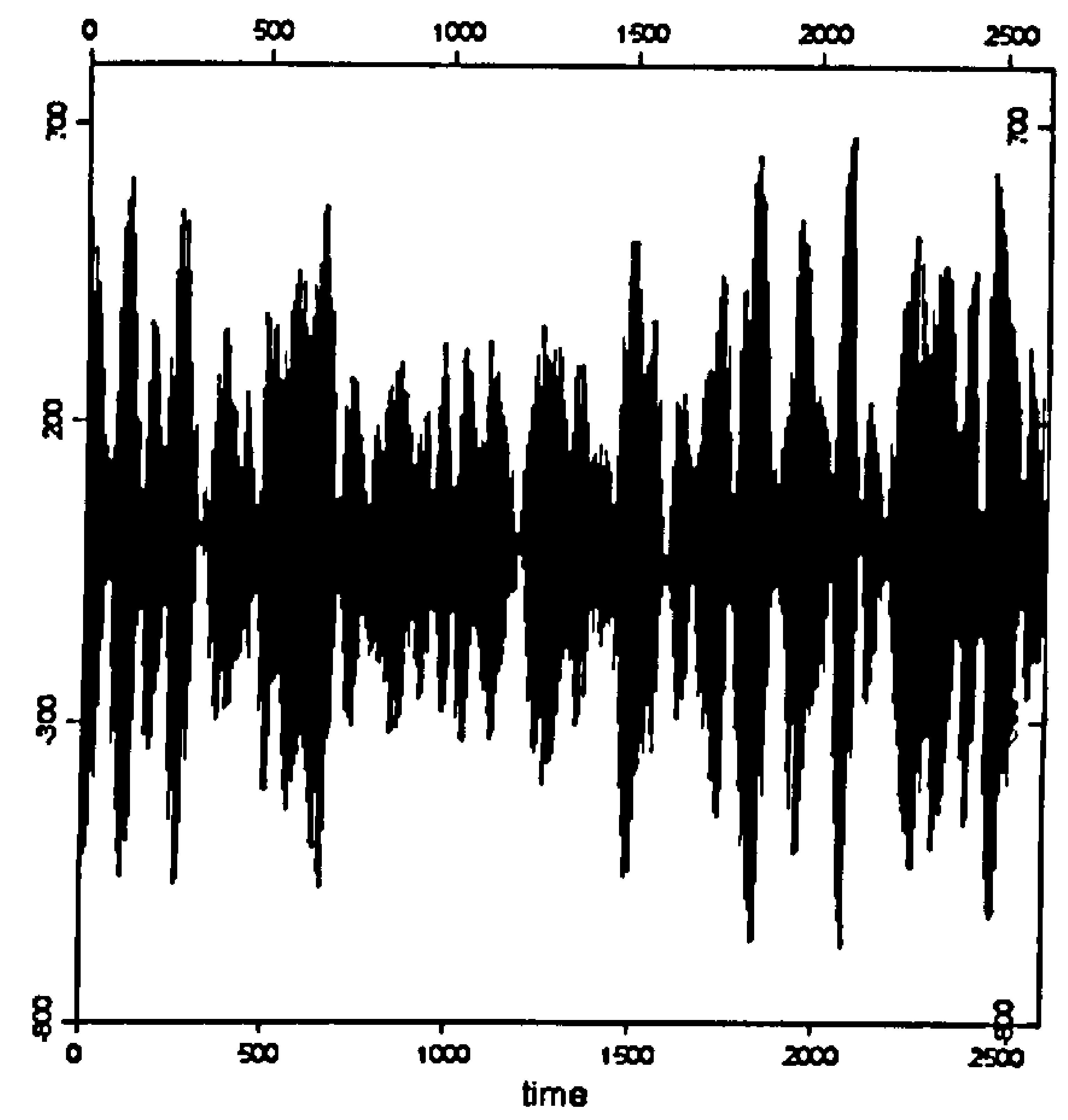

Figure 2.19: Imaginary part of the last 2609 data points of the PISCES file EB0316.008.

Another interesting example is given by the periodogram of the file EB0327.021 (figure 2.20, measurement taken at 21pm on 27 March 1987). Figure 2.21 exhibits its scalogram where peaks show up only at the beginning of the record. If we look at this file, we notice that the first 700 samples are much larger in amplitude than the rest. Figures 2.22 and 2.23 show respectively the periodogram and the AR PSD estimate (obtained by the modified covariance method as well), with $N=700$. They give a satisfactory estimation of the frequency difference, $\Delta f=0.532227 \mathrm{~Hz}$ $\left(\Delta f_{t}=0.533065 \mathrm{~Hz}\right)$ and the level of the noise has reduced by about $10 \mathrm{~dB}$. 


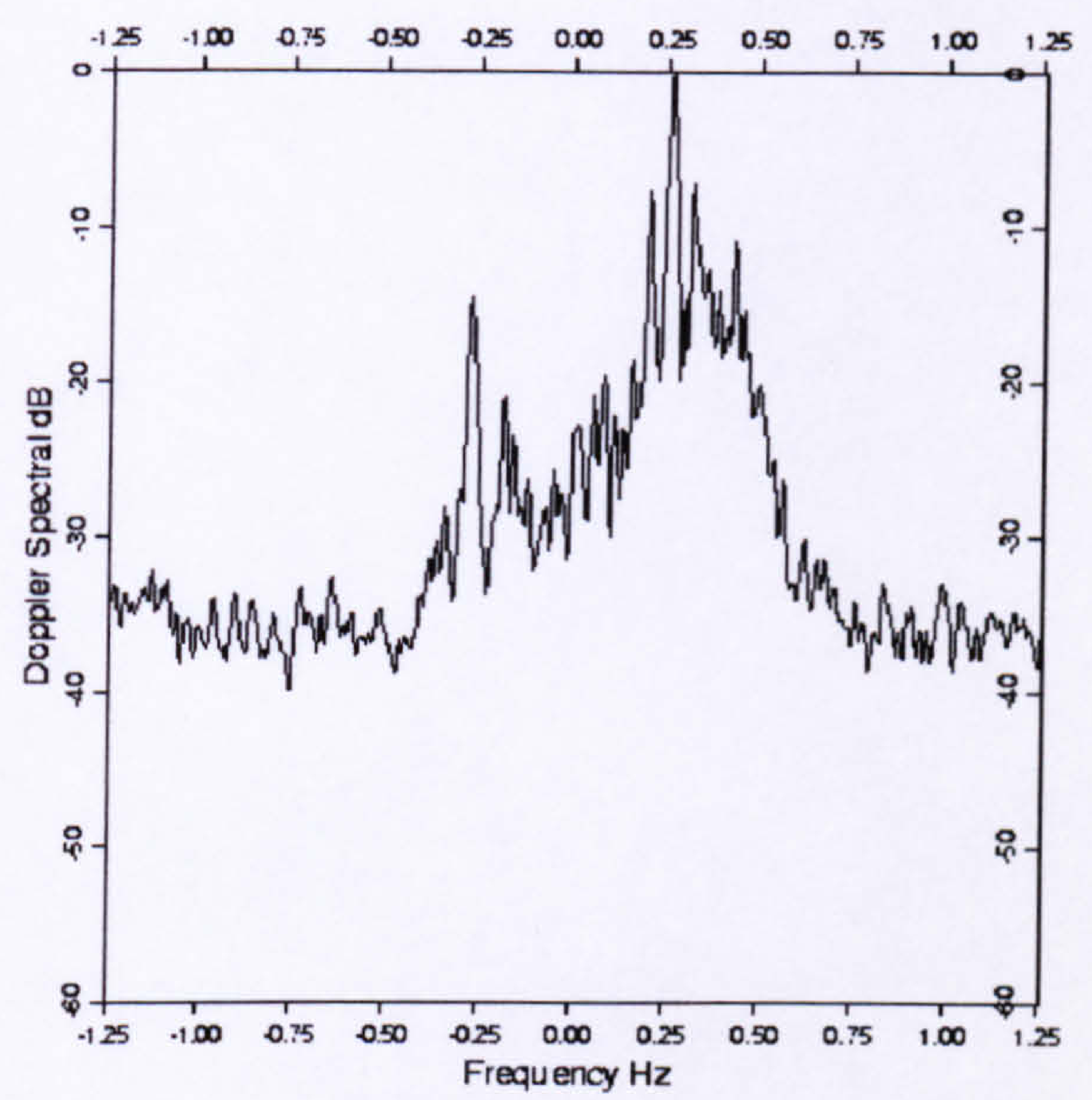

Figure 2.20: Periodogram of the PISCES file EB0327.021

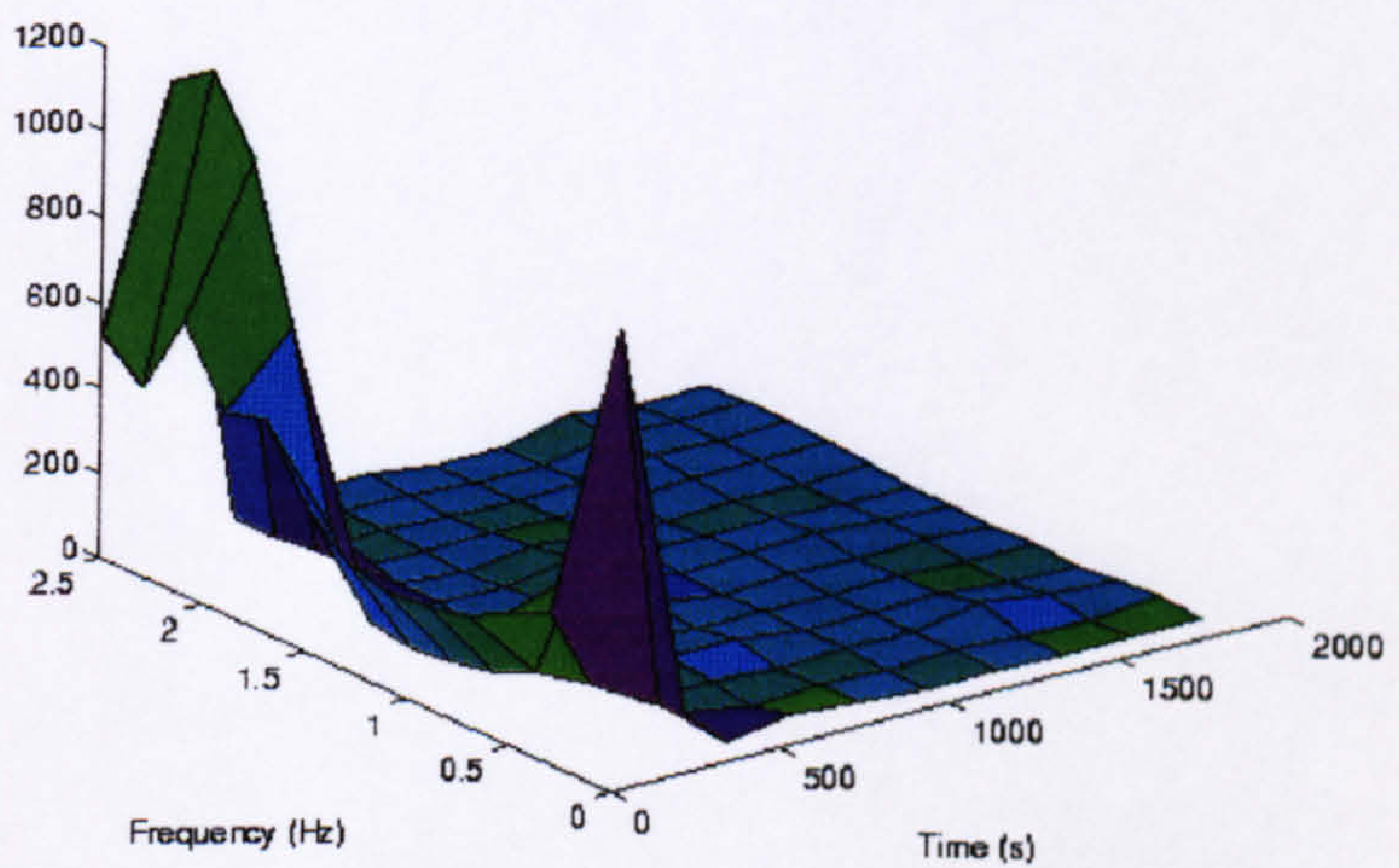

Figure 2.21: Morlet scalogram of the PISCES file EB0327.021. 


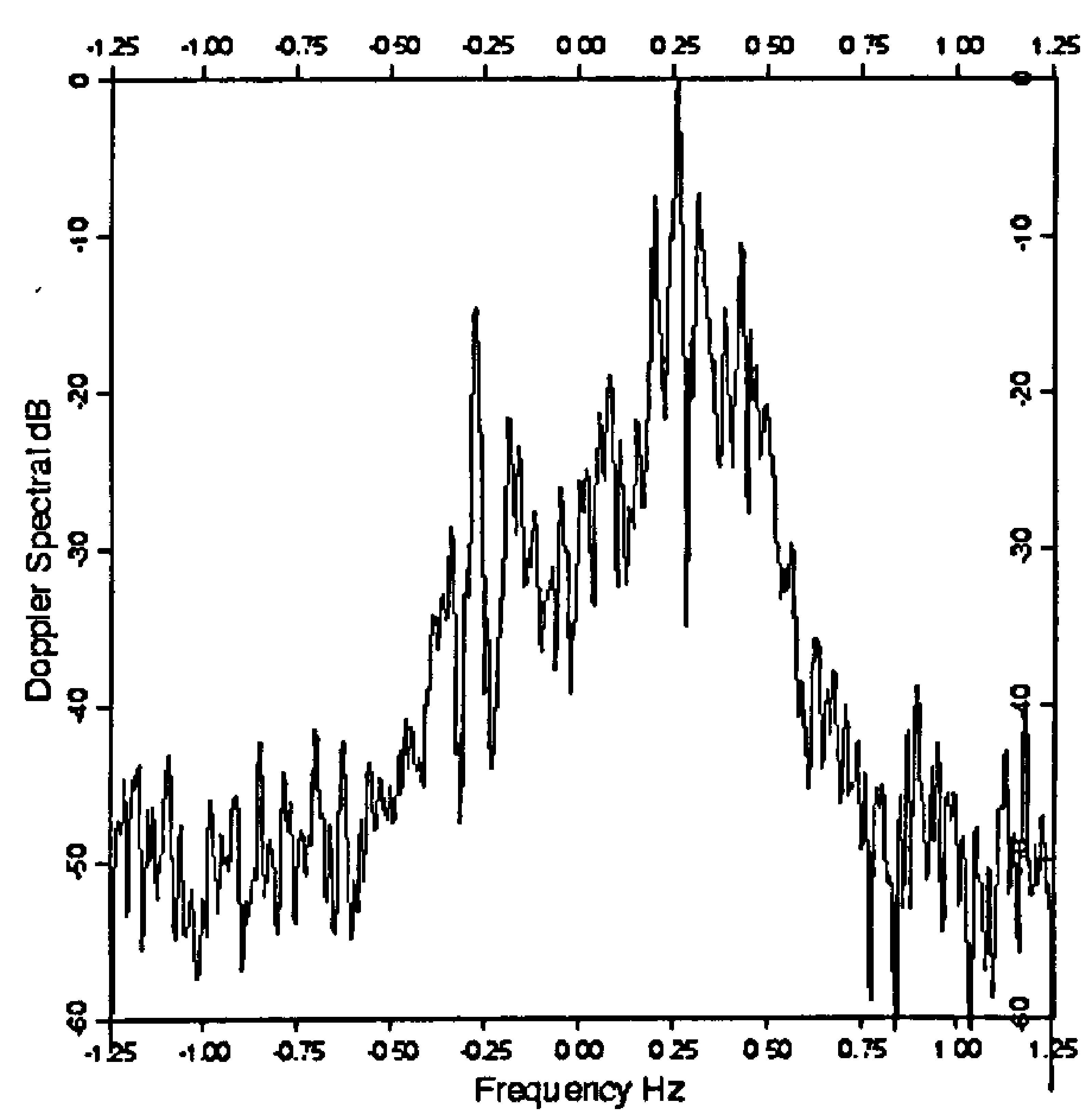

Figure 2.22: Periodogram of the PISCES file EB0327.021 using only the first 700 data points.

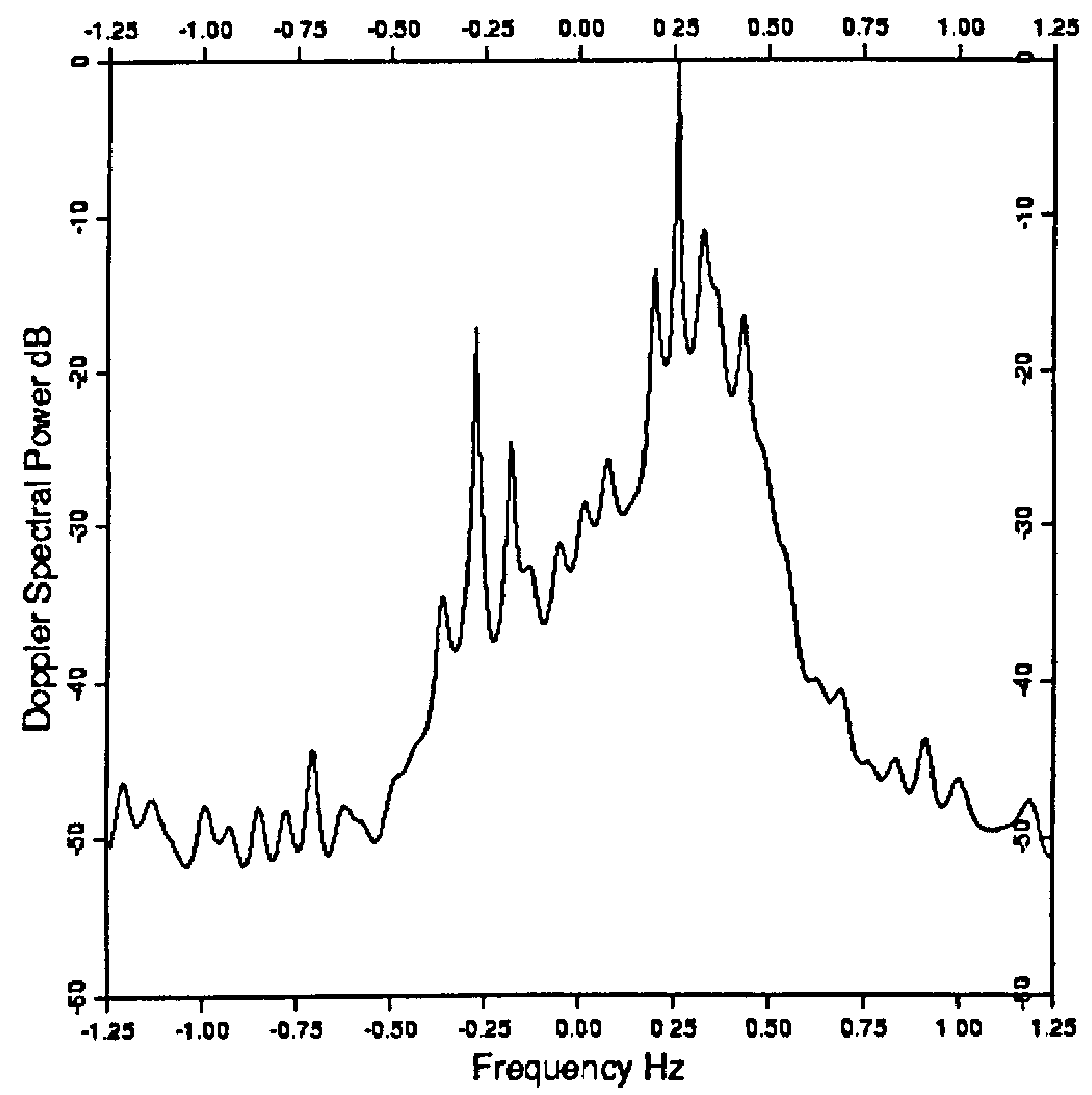

Figure 2.23: AR PSD estimate of the PISCES file EB0327.021 with $N=700$ and $p=40$. 
The last example we propose concerns the problem of split peaks shown in figure 1.2. Figure 2.24 exhibits the scalogram of the same file originating from the OSCR system. The measurement was taken at $6 \mathrm{pm}$ on 6 January 1996 from the cell 55 . As it can be seen, two rows appear as for the previous cases with the PISCES system. If we zoom into the region containing the most powerful frequencies, figure 2.25 (a), with the top view (b), we can see clearly that the Bragg frequency has moved during the hour measurement. We can also notice there is an anomaly during the period of time 600 700s; no signals show up.

We can see already that the wavelet analysis is useful and promising. It can tell us when the information is the most reliable and show the quality of the data. It reveals lack of stationarity and emphases the importance of using modern spectral methods.

Morlet Scalogram

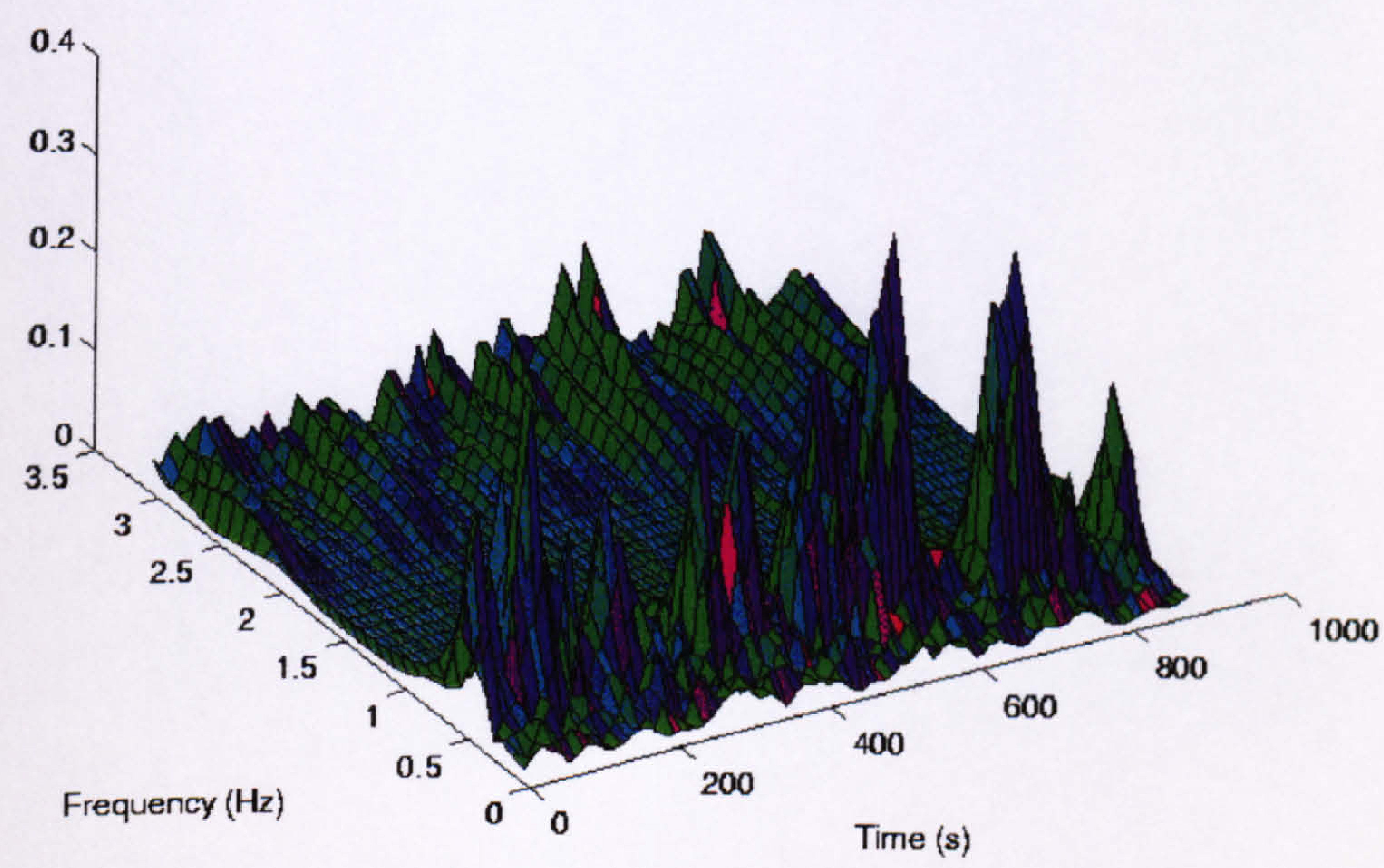

Figure 2.24: Morlet scalogram obtained from the same OSCR file as shown in figure 1.2 (cell 55, measurement taken at 6pm on 6 January 1996). 


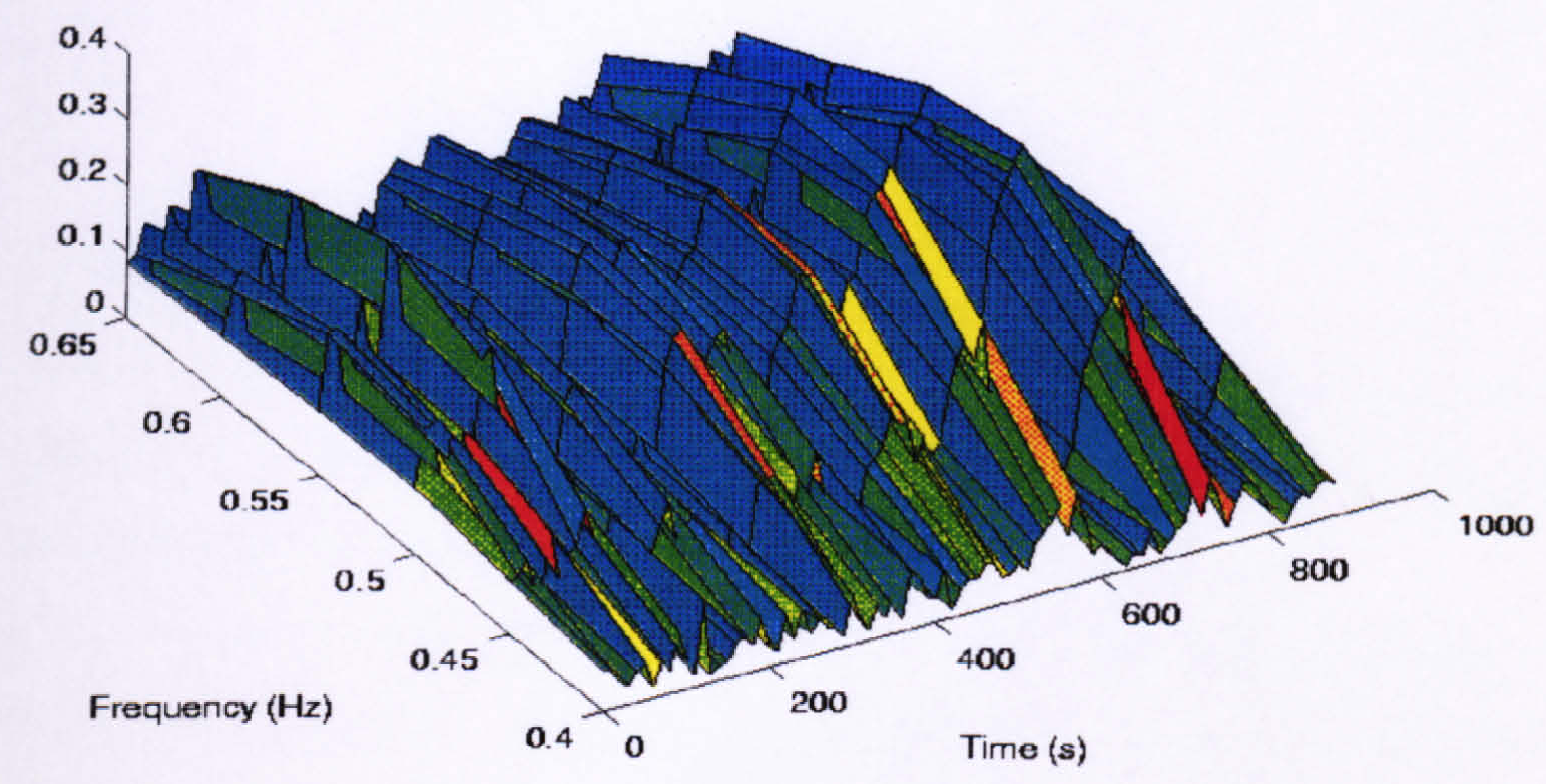

(a)

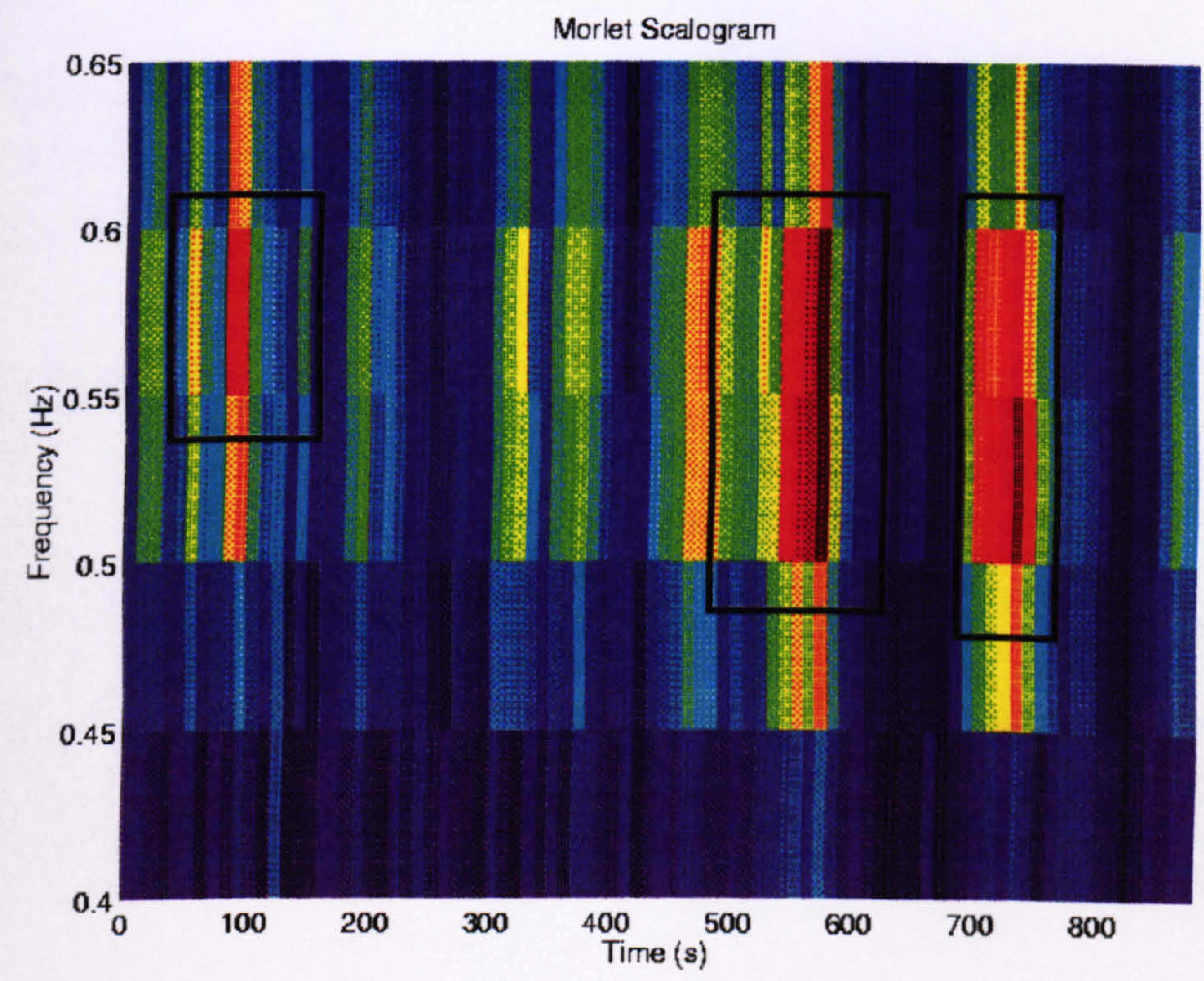

(b)

Figure 2.25: Morlet scalogram obtained from the same OSCR file as shown in figure 1.2 (cell 55, measurement taken at $6 \mathrm{pm}$ on 6 January 1996), showing the frequences from $0.4 \mathrm{~Hz}$ to $0.7 \mathrm{~Hz}$, side view (a) and top view (b). The rectangular highlights in (b) show the moving Bragg frequency. 


\section{Chapter 3}

\section{Modern Spectral Estimation}

This section provides a review of techniques for the modern spectral estimation. We are interested in finding the most appropriate to our applications in other words HF radar systems for ocean monitoring.

The motivation of using such methods is the use of short data sets while providing stable spectral estimates. In order to select the most efficient and reliable modern spectral method, the different methods are tested on the data collected by the PISCES system. Some methods are limited by the amount of data which can be used, which leads us to take data sets of 100 samples. In this chapter, we just consider the firstorder effect. We look at the position of the two main Bragg peaks and compare the total frequency difference to the theoretical one (see equation (2.11)). The secondorder contribution will be investigated in the following chapters.

Four different spectral estimations are described and applied: 1. the autoregressive spectral estimation suitable for spectra with sharp peaks; 2 . the autoregressive moving average spectral estimation able to represent different spectral shapes; 3 . the eigenanalysis-based frequency estimation producing peaks at narrowband spectra and 4. the recursive maximum likelihood estimation (RMLE) which is restricted to real data. 


\subsection{Historical review}

The ocean wave measurements are obtained from the periodogram based on the FFT processing. This classical spectral technique was introduced by Shuster (1898). In 1927, Yule proposed an alternative analysis method by performing a least squares autoregression analysis of the following model :

$$
u(k)=b(1) u(k-1)+b(2) u(k-2)+\varepsilon(k),
$$

with $\varepsilon(k)$ small random error at each time $k, b(1)$ and $b(2) 2$ arbitrary values and $u(k)$ the signal, in order to search for any periodicities in the data.

The solution of this regression equation is a damped sinusoid. The normal equations resulting from this analysis have been called the Yule-Walker equations. Walker (1931) used this technique to investigate damped sinusoidal time series.

The year 1930 marked a major turning point for spectral analysis. It was Wiener who, by publishing his classic paper "Generalized Harmonic Analysis" (1930) placed spectral analysis on a firm statistical basis in its treatment of random processes. Precise statistical definitions of autocorrelation and power spectral density (PSD) were given for stationary random processes. These two functions of a random process were shown to be related via a continuous Fourier transform, which is the basis of the Wiener-Khintchine theorem. Wold (1938) introduced the terms moving average and linear autoregression for time-series model. He was the first to describe the relationship between the autoregressive parameters and the autocorrelation sequence as the Yule-Walker equations. He also introduced a very important decomposition theorem for stationary time series. It asserted that any stationary random process could be expressed as the sum of a deterministic component and a one-sided movingaverage process driven by a white noise. This theorem led Kolmogoroff (1939) to formulate and solve the linear prediction problem. In 1948, Barlett suggested the computation of a spectrum from the autoregressive coefficients.

The prime motivator for the current interest in high-resolution spectral estimation from limited data sets may be attributed to Burg (1967). He developed the maximum entropy spectral analysis which is related to autoregressive spectral analysis. Since then, many modern spectral analysis methods have been developed because of their ability to provide high frequency resolution from short data sets (Marple, 1987 and 
Kay, 1988). Figure 3.1 shows an example of this ability. Two power spectra are presented, both resulted from a sum of two complex exponential functions plus a noise term. One is obtained by the conventional FFT and the other one by the Burg method. As it can be seen, the two peaks are thinner with the Burg method; higher frequency resolution is obtained.

The fact that fast algorithms have been developed is an important consideration for real-time operation.

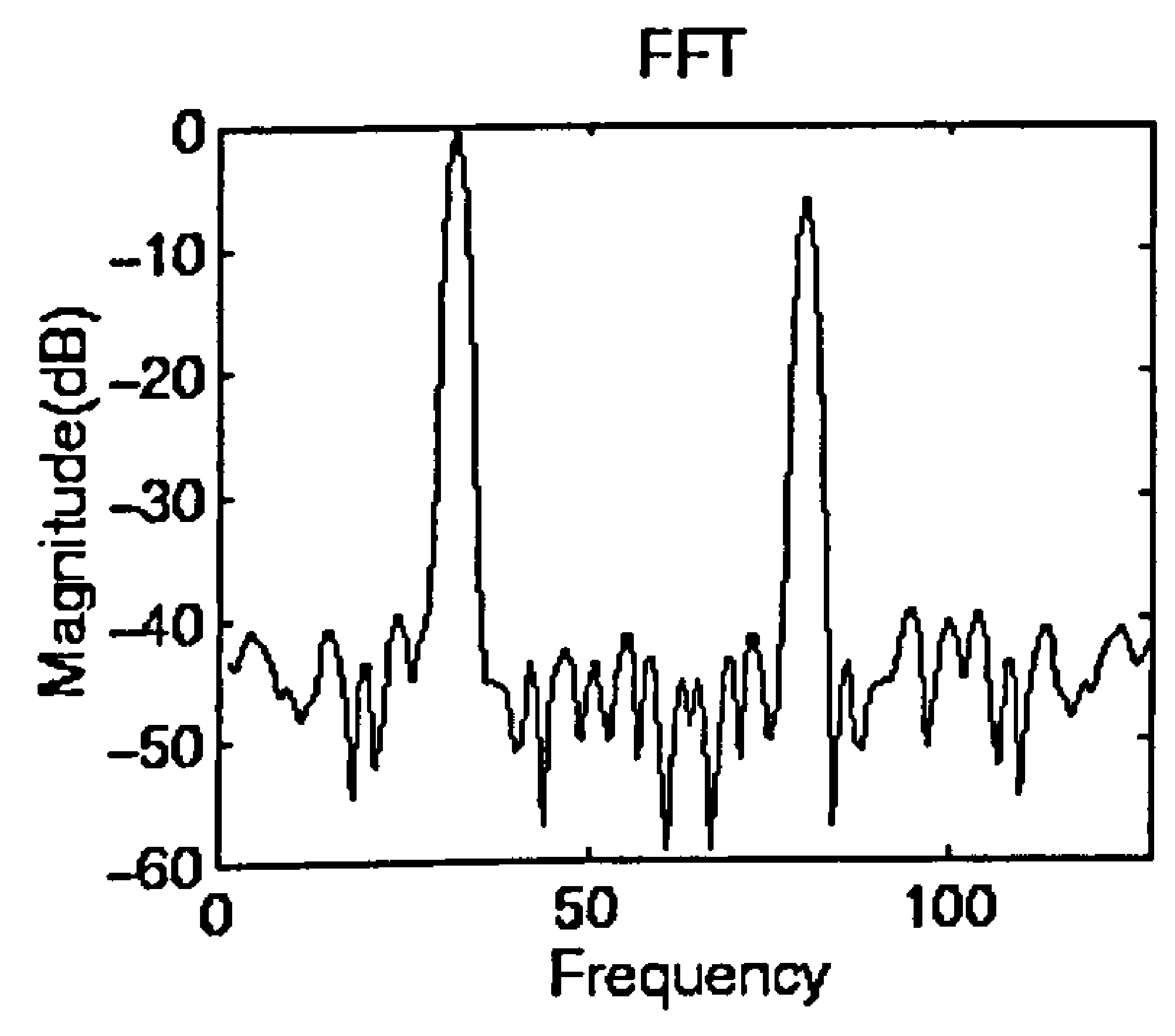

(a)

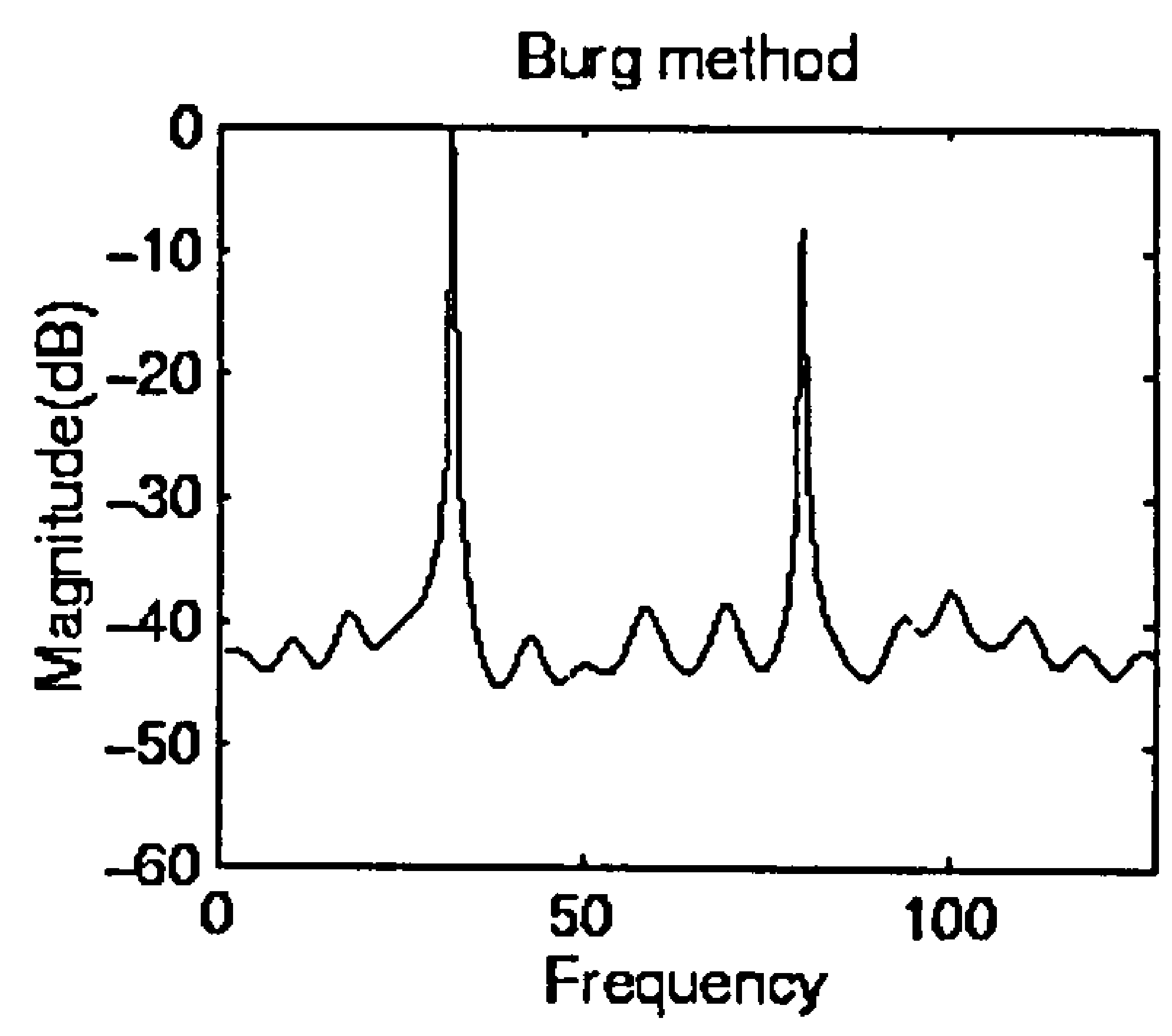

(b)

Figure 3.1: Power spectra over 128 frequency points resulted from the FFT (a) and the Burg method (b), showing the differences in the frequency resolution.

\subsection{Autoregressive Spectral Estimation}

Let $\{x(1), \ldots, x(N)\}$ be $N$ consecutive samples of the time series of ocean backscatter at one range cell.

The observed sequence $\{x(1), \ldots, x(N)\}$ is assumed to be the output from a $p$ th order autoregressive (AR) model driven by a white noise process $u(n)$, in other words the current output sample $x(n)$ is assumed to be a weighted sum of $p$ past output samples plus a noise term: 


$$
x(n)+\sum_{k=1}^{p} a_{k} x(n-k)=u(n)
$$

where $a_{k}$ is AR parameter $k$ of a $p$ th order AR process.

The linear prediction problem is simply stated as follows. Given the observations $x(s), s \leq n$, we wish to estimate the next sample $x(n), n>0$. Consequently, the $\operatorname{AR}(p)$ process is a linear prediction problem.

Our objective is to compute the AR power spectral estimate $\underset{A R}{p}(f)$ (Marple, 1987) once the AR parameters have been estimated:

$$
\hat{p} \underset{A R}{\wedge}(f)=\frac{T \hat{\rho}}{\left|1+\sum_{n=1}^{p} \hat{\mathrm{a}}_{\mathrm{p}}(n) e^{-2 j \pi f n T}\right|^{2}}
$$

where $T$ is the sample interval,

$\hat{\rho}$ is an estimate of the driving noise variance,

and $\hat{a}_{p}(n)$ are the AR parameter estimates.

To do so, we have computed the covariance and the modified covariance methods which are based on a least squared linear prediction approach and the Burg method which estimates the reflection coefficients (Marple, 1987, p 195 and 213). These coefficients are provided by the Levinson recursion :

$$
a_{p}(n)=a_{p-1}(n)+k_{p} a_{p-1}^{*}(p-n),
$$

where $k_{p}=a_{p}(p)$.

All these methods are described by Marple (1987) and Proakis-Manolakis (1992). The modified covariance method is fully described in the next chapter. In Appendix $\mathrm{B}$, a brief description of the Burg method is given.

For the location of the two main peaks, the modified covariance and Burg methods give similar results on the PISCES data. For example, figures 3.2 and 3.3 show the AR PSD estimates of the PISCES file EB0328.008 (measurement taken at 11 am on 28 March 1987). The estimated frequency difference is the same for these two techniques: $\Delta f=0.620117 \mathrm{~Hz}$ and the theoretical frequency difference is: $\Delta f_{t}=0.620583 \mathrm{~Hz}$. 
When the difference between $\Delta f$ and $\Delta f_{t}$ is smaller than one frequency bin, $h=\frac{\Delta F}{N}=\left\{\begin{array}{c}0.00594 \text { (OSCR system) } \\ 0.00488 \text { (PISCES system) }\end{array}\right.$ where $\Delta F$ is the frequency range and $N$ the number of frequency points, we assume that " $\Delta f$ is close to $\Delta f_{t}$ ". This is the case for the last examples.

Marple (1987, p224-229) describes estimator characteristics and the different anomalies met in the autoregressive spectra. The main difference between the Burg and the modified covariance techniques is that in the Burg method the phenomenon of spectral line splitting can occur. This phenomenon is not the same problem mentioned in the Introduction. Figure 3.4 exhibits a Burg spectral estimate that has split the first main peak into two peaks and figure 3.5 exhibits a modified covariance spectral estimate that does not contain line splitting at the same frequency. Herring (1980) studied the cause of line splitting in the Burg method. In the case of two complex sinusoids in high signal-to-noise ratio (SNR) of 40-50dB, it was shown that it is difficult to explain this phenomenon. No evidence of line splitting has ever been observed with the modified covariance method (Marple, 1987, p226).

Therefore, we prefer to work with the modified covariance method because it is more reliable in this aspect.

\subsection{Autoregressive Moving Average Spectral Estimation}

The AR model is by far the most widely used (see previous section). It is suitable for representing spectra with narrow peaks, it results in very simple linear equations and the decomposition theorem due to Wold (1938) asserts that any autoregressive moving average (ARMA) or moving average (MA) process may be represented uniquely by an AR model of possibly infinite order. 


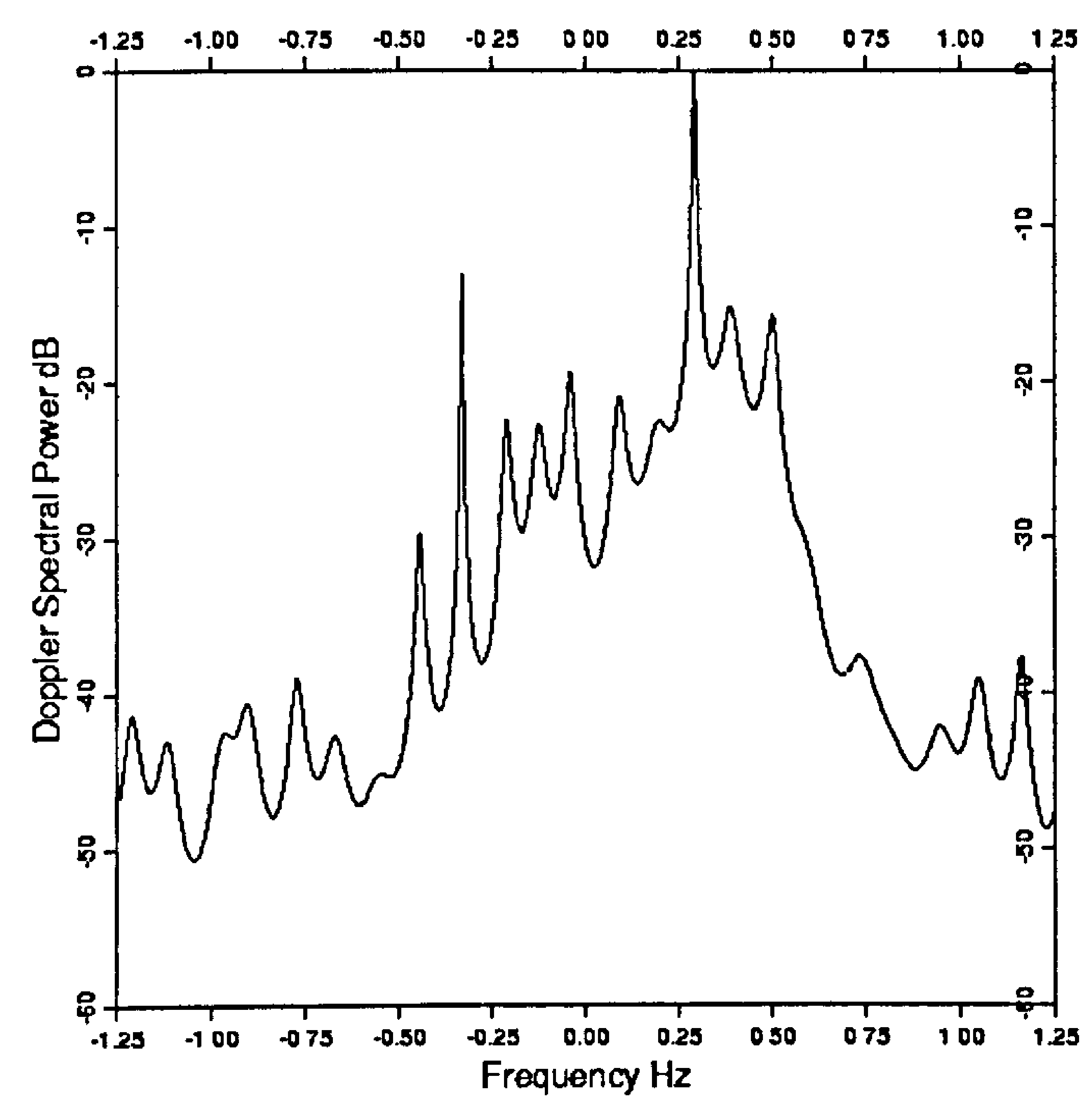

Figure 3.2: Doppler spectrum of the PISCES file EB0328.008, obtained by the modified covariance method with $N=100$ and $p=25$.

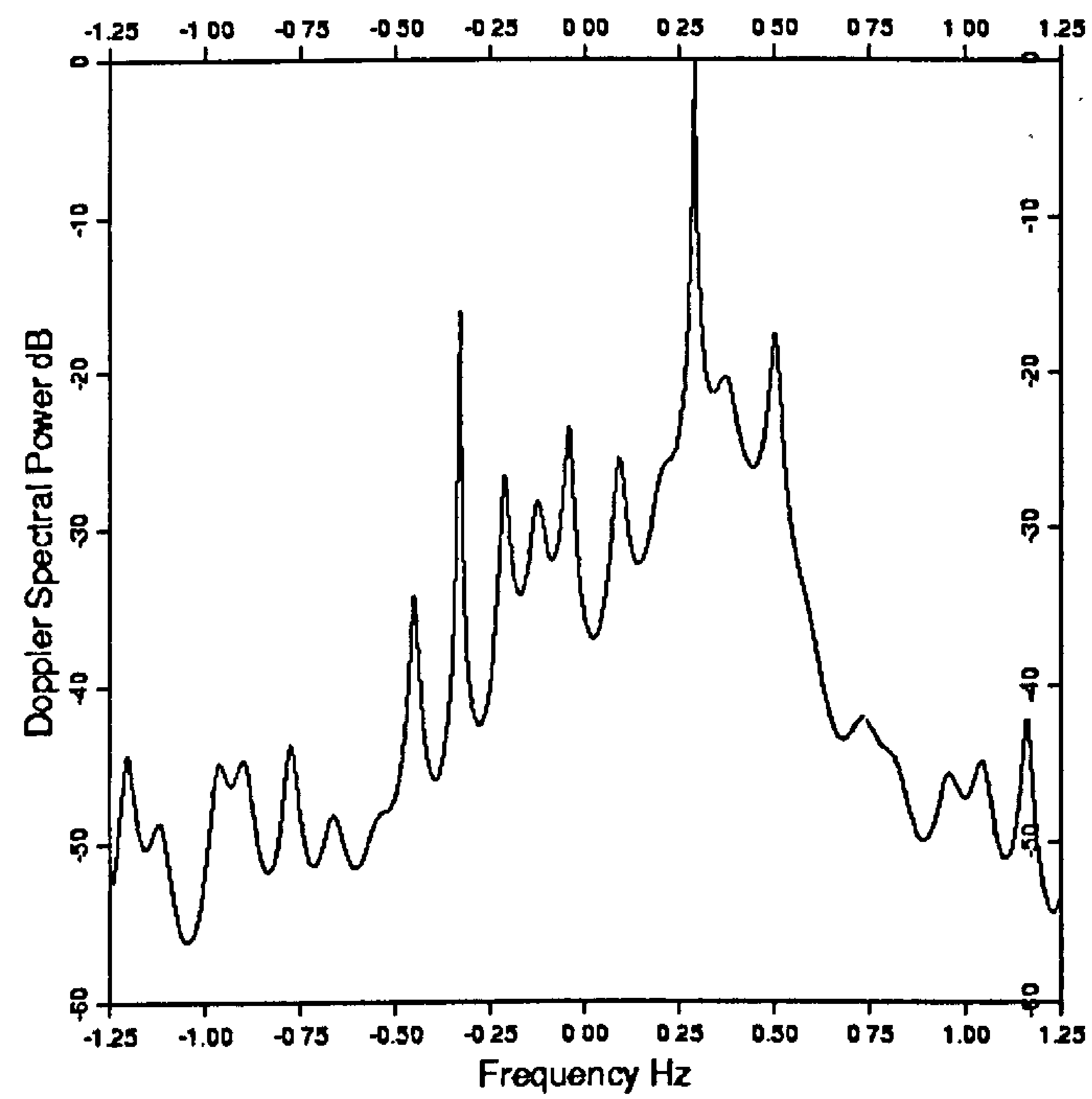

Figure 3.3: Doppler spectrum of the PISCES file EB0328.008, obtained by the Burg method with $N=100$ and $p=25$. 


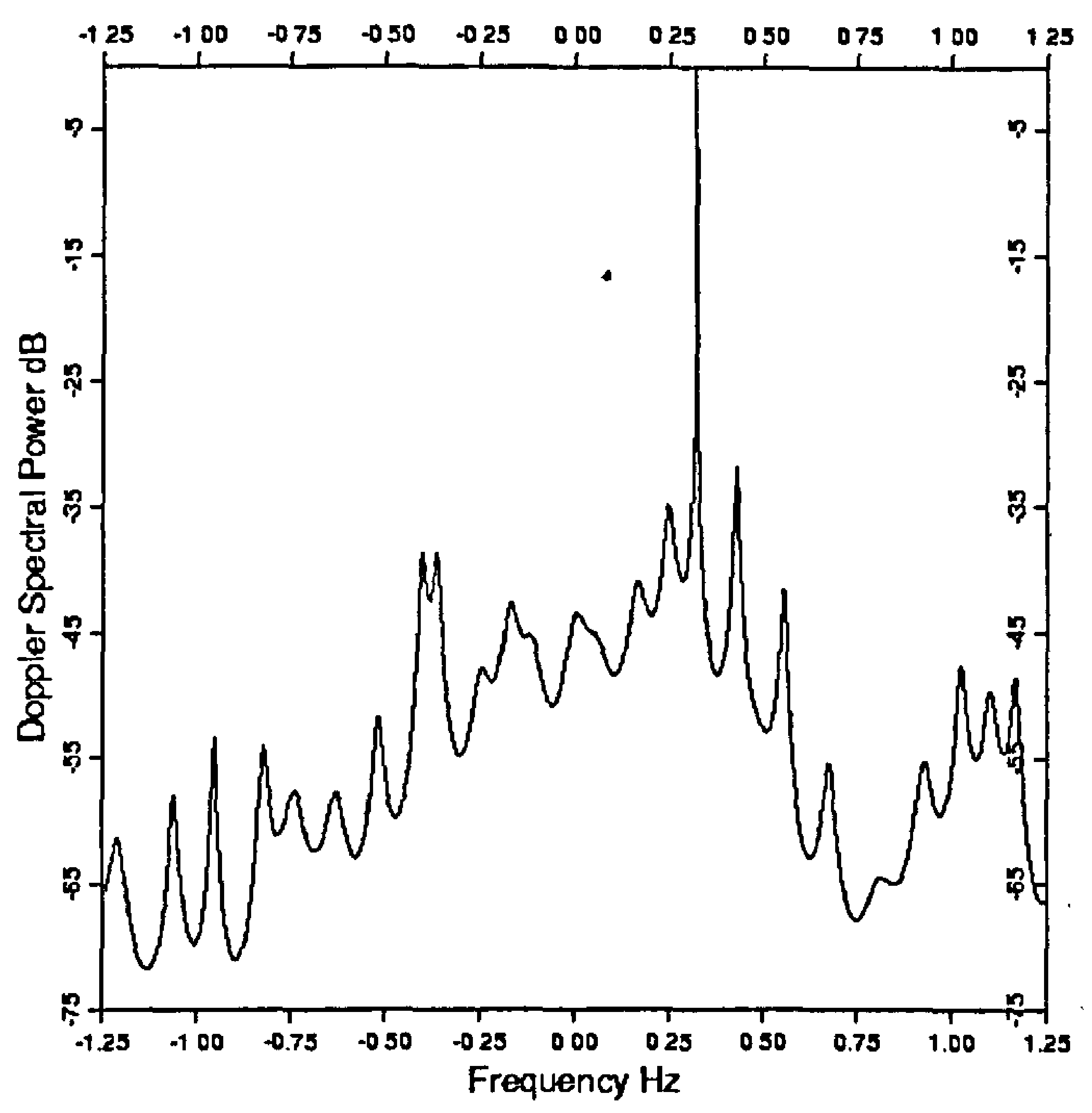

Figure 3.4: Doppler spectrum obtained by the Burg method using the file EB0329.011 with $N=90$ and $p=30$. The largest negative frequency peak between $-0.50 \mathrm{~Hz}$ and $-0.25 \mathrm{~Hz}$ splits into two peaks.

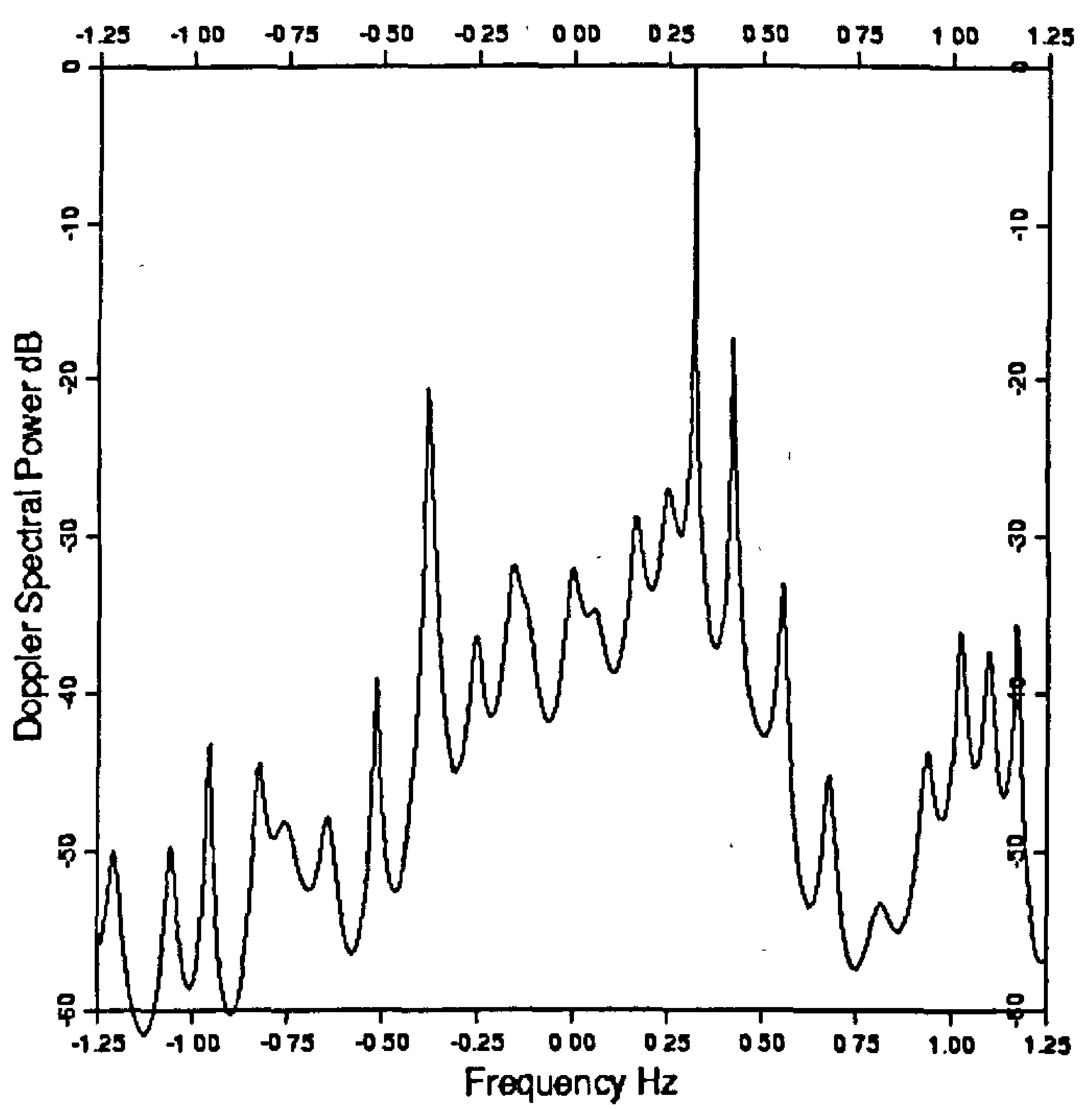

Figure 3.5: Doppler spectrum obtained by the modified covariance method using the file EB0329.011 with $N=90$ and $p=30$. No line splitting occurs. 
However, the ARMA model can generate diverse spectral shapes and it may be appropriate to our case. It can be expressed by:

$$
x(n)=-\sum_{k=1}^{p} a_{k} x(n-k)+\sum_{k=0}^{p} b_{k} u(n-k)=\sum_{k=0}^{\infty} h(k) u(n-k),
$$

where $a_{k}$ is the AR parameter $k$ of a $p$ th order AR process, $b_{k}$ the MA parameter $k$ of a $q$ th order MA process and $h(k)$ is a casual filter with $h(0)=1$.

Unlike the extensive range of algorithms to produce AR PSD estimates, there have been few algorithms developed for ARMA PSD estimates. This is due to the difficulty of estimating simultaneously the MA and AR parameters of the ARMA model. Iterative optimization techniques have been developed to solve the nonlinear equation obtained for the autocorrelation sequence $r_{x x}(m)=E\left(x(n+m) x^{*}(n)\right)(E$ being the expectation) :

$$
r_{x x}(m)=\left\{\begin{array}{c}
r_{x x}^{*}(-m) \text { for } m<0 \\
-\sum_{k=1}^{p} a_{k} r_{x x}(m-k)+\rho_{w} \sum_{k=0}^{q} b_{k} h^{*}(k-m) \text { for } 0 \leq m \leq q . \\
-\sum_{k=1}^{p} a_{k} r_{x x}(m-k) \text { for } m>q
\end{array}\right.
$$

But these techniques involve important computations and are not guaranteed to converge. Therefore, we implemented a suboptimum technique (Marple, 1987, p 285). This technique estimates the $A R$ and the MA parameters separately in 3 steps :

1. estimation of the $\mathrm{AR}$ parameters with the covariance method, $a_{1}, \ldots, a_{p}$ from the original data based on a least-squares ARMA Yule-Walker technique.

2. production of a residual time sequence by filtering the data with a filter based on the last AR parameters,

3. estimation of the MA parameters from the residual time sequence $b_{0}, \ldots, b_{q}$. The ARMA Yule-Walker equations (Marple, 1987, p 182) describe the relationship between the autoregressive parameters of the ARMA model and the autocorrelation sequence.

Then, the ARMA PSD estimate can be evaluated: 


$$
p_{A R M A}(f)=T \rho_{w}\left|\frac{\sum_{k=0}^{q} b_{k} \exp (-2 j \pi k f T)}{1+\sum_{l=1}^{p} a_{l} \exp (-2 j \pi l f T)}\right|^{2},
$$

where we have taken small values of $p=q$.

When $p$ is too large, we find that the total frequency difference is biased:

$\Delta f(p=5)=0.620117 \mathrm{~Hz}$ (see fig. 3.6) and $\Delta f(p=25)=0.751953 \mathrm{~Hz}$ (see fig. 3.7)

Figures 3.6 and 3.7 are obtained from the file EB0319.011.

This method is comparable to the AR methods with taking small values of $p$. Good estimation of the frequencies are obtained. Figure 3.6 exhibits the ARMA PSD estimate and its total difference frequency estimate, $\Delta f=0.620117 \mathrm{~Hz}$, is close to the theoretical one, $\Delta f_{t}=0.620583 \mathrm{~Hz}$. But it is preferable to use the Burg or the modified covariance method in view of fact that the ARMA method is limited by the orders $p$ and $q$ and its computation takes (at least) twice the time of the modified covariance method (Marple, 1987).

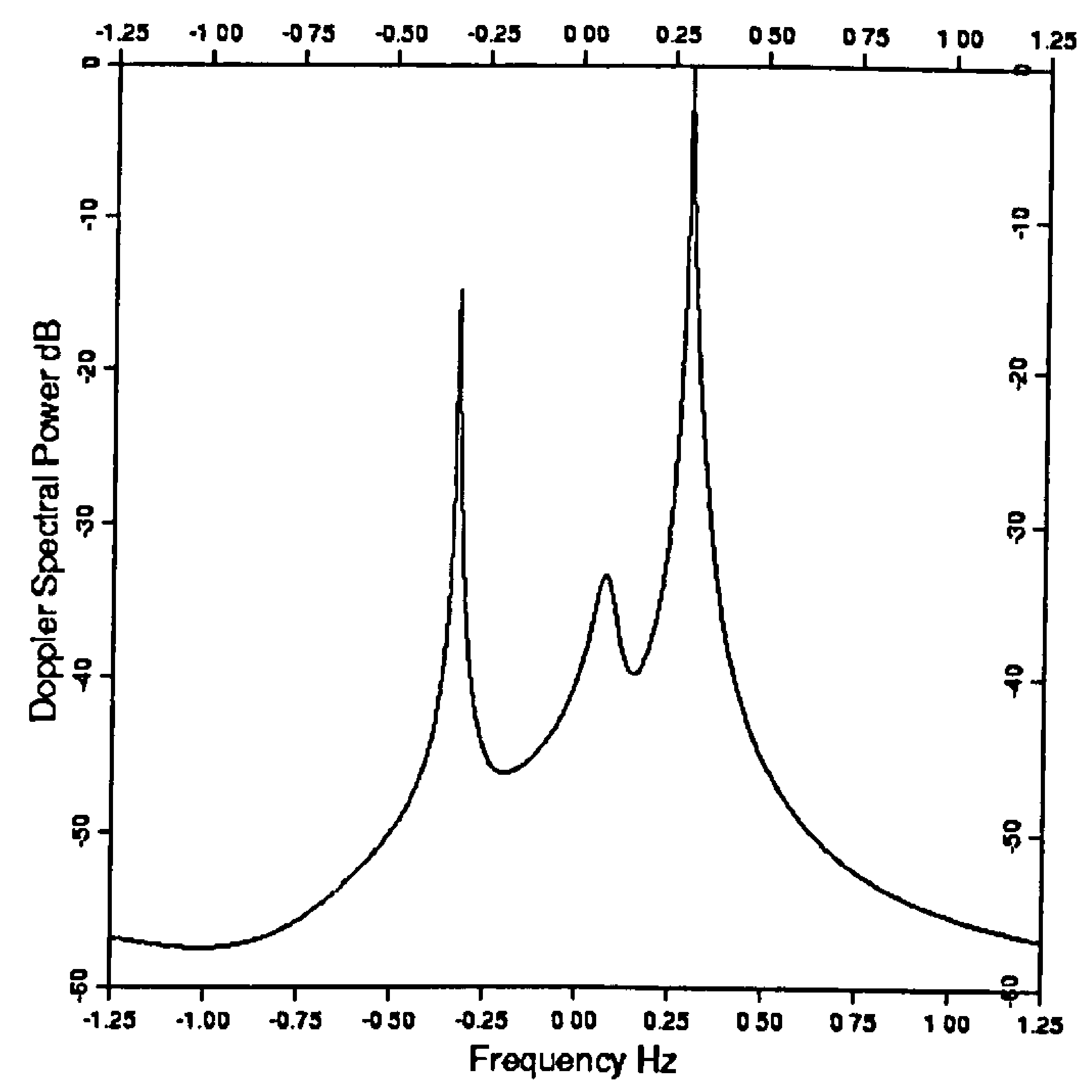

Figure 3.6: Doppler Spectrum of the PISCES file EB0319.011 obtained by an ARMA model with $N=100$ and $p=q=5$. 


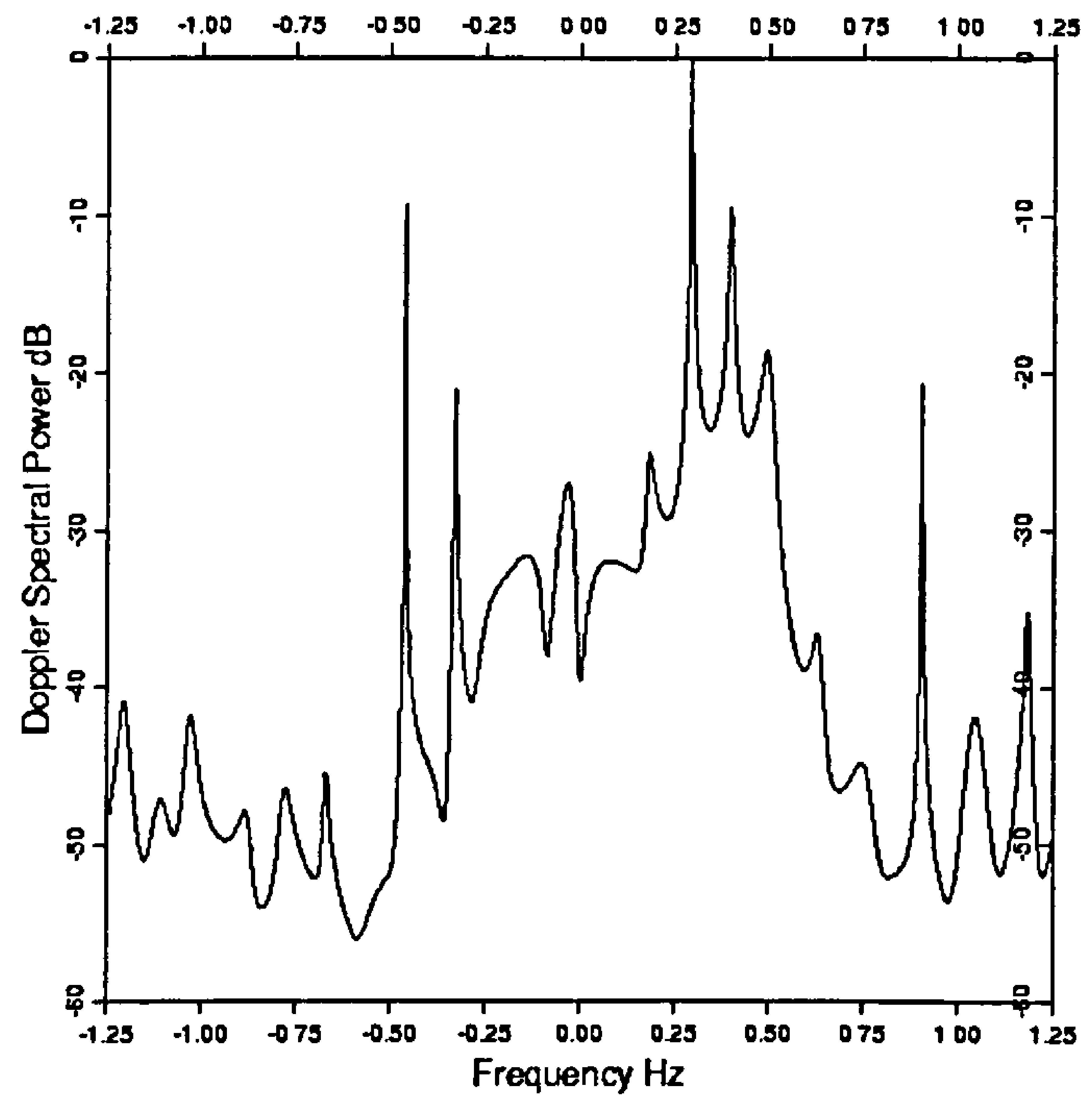

Figure 3.7: Doppler Spectrum of the PISCES file EB0319.011 obtained by an ARMA model with $N=100$ and $p=q=25$.

\subsection{Eigenanalysis-Based Frequency Estimation}

This method described in Marple (1987, p 361) and Proakis et al. (1992, p 278), divides the information in the autocorrelation matrix $R_{p}$ of a random process $\{x(n)\}$ into 2 vectors subspaces, one a signal subspace and the other a noise subspace:

$$
R_{p}=\left[\begin{array}{ll}
r_{x x}(0) & \ldots r_{x x}^{*}(p) \\
r_{x x}(p) & \ldots r_{x x}(0)
\end{array}\right],
$$

where $r_{x x}(m)=E\left(x(n+m) x^{*}(n)\right)$

This creates frequency estimators and their plots show sharp peaks at the frequency locations of sinusoids or narrowband spectral components.

These frequency estimators are not true PSD estimators because they do not preserve the measured process power. Therefore, this method is only worthwhile for the estimated frequency difference between the two main peaks. 
Hickey et al. (1995) have used this technique in order to get radar surface current measurements, and good results (compared with the conventional FFT-based method) have been obtained.

Here is a view of the eigen-decomposition method for sinusoids in white noise:

$$
x(n)=\sum_{i=1}^{p} A_{i} e^{j\left(2 \pi f_{i} n+\phi_{i}\right)}, n=1 . . N
$$

where the amplitudes $\left\{A_{i}\right\}$ and the frequencies $\left\{f_{i}\right\}$ are unknown and the phases $\left\{\phi_{i}\right\}$ are statistically independent random variables uniformly distributed on $(0,2 \pi)$. Then, $x(n)$ is wide-sense stationary with the autocorrelation function

$$
\gamma_{x x}(m)=\sum_{i=1}^{p} p_{i} e^{2 j \pi f_{i} m}
$$

The observed sequence is

$$
y(n)=x(n)+w(n)
$$

where $w(n)$ is a white noise sequence with spectral density $\sigma_{w}^{2}$. Its autocorrelation function is

$$
\gamma_{y y}(m)=\gamma_{x x}(m)+\sigma_{w}^{2} \delta(m), m=0, \pm 1, . \pm(n-1)
$$

and its autocorrelation matrix is

$$
\Gamma_{y y}=\Gamma_{x x}+\sigma_{w}^{2} \mathrm{I}
$$

where $\Gamma_{x x}$ is the autocorrelation matrix for $x(n)$ of rank $\mathrm{p}$ :

$$
\Gamma_{x x}=\sum_{i=1}^{p} p_{i} s_{i} s_{i}^{H}, s_{i}=\left[1, e^{2 j \pi f_{i}}, \ldots, e^{2 j \pi(n-1) f_{i}}\right]
$$

where $H$ denotes the Hermitian transpose operation.

Now, we are ready to do the eigen-decomposition of $\Gamma_{y y}$. Let the eigenvalues $\left\{\lambda_{i}\right\}$ be ordered in decreasing value with $\lambda_{1} \geq \lambda_{2} . . \geq \lambda_{M}, M$ being the rank of $\Gamma_{y y}$ and $M \geq p$. And let the corresponding eigenvectors be denoted as $\left\{v_{i}, i=1 . . M\right\}$.

Assume that

$$
v_{i}^{H} v_{j}=\delta_{i j}
$$

In the absence of noise, we have

$$
\left\{\begin{array}{c}
\lambda_{i} \neq 0, i=1 . . p \\
\lambda_{p+1}=\lambda_{p+2} . .=\lambda_{M}=0
\end{array} \text { and } \Gamma_{x x}=\sum_{i=1}^{p} \lambda_{i} v_{i} v_{i}^{H}\right.
$$


The vectors $v_{i}, i=1 . . p$ are called the principal eigenvectors and the values $\lambda_{i}, i=1 . . p$ the principal eigenvalues.

Also, we can write that $\quad \sigma_{w}^{2} \mathrm{I}=\sigma_{w}^{2} \sum_{i=1}^{M} v_{i}^{H} v_{i}$

Then, $\quad \Gamma_{y y}=\sum_{i=1}^{p}\left(\lambda_{i}+\sigma_{w}^{2}\right) v_{i} v_{i}^{H}+\sum_{i=p+1}^{M} \sigma_{w}^{2} v_{i} v_{i}^{H}$.

The set $\left\{v_{i}, i=1 . . p\right\}$ represents the signal subspace and $\left\{v_{i}, i=p+1 . . M\right\}$ the noise subspace which is orthogonal to the principal eigenvectors. Therefore, the vectors $\left\{s_{i}\right\}$ are linear combinations of the principal eigenvectors and are orthogonal to the vectors in the noise subspace.

Consider the weighted spectral estimate:

$$
P(f)=\sum_{k=p+1}^{M} w_{k}\left|s^{H}(f) v_{k}\right|^{2}
$$

where $\left\{v_{k}, k=p+1 . . M\right\}$ are the eigenvectors in the noise subspace, $\left\{w_{k}\right\}$ the set of positive weights and $s(f)=\left[1, e^{2 j \pi f}, \ldots, e^{2 j \pi(M-1) f}\right]$ the complex sinusoidal vector.

Note that at $f=f_{i}, s\left(f_{i}\right)=s_{i}$, we have $P\left(f_{i}\right)=0, i=1 . . p$. Then $1 / P(f)$ can estimate the frequencies of the sinusoidal components.

The MUSIC (stand for MUltiple SIgnal Classification) sinusoidal frequency estimator is a special case in which $w_{k}=1, \forall k$ :

$$
P_{M U S I C}(f)=\frac{1}{\sum_{k=p+1}^{M}\left|s^{H}(f) v_{k}\right|^{2}} \text {. }
$$

The estimate of the sinusoidal frequencies are the peaks of $P_{\text {MUSIC }}(f)$.

In our case, we would prefer to get two peaks. 20 tests with the PISCES data have showed that we can get a good estimation of the most important peak. For example, figure 3.8 (file EB0319.011, measurement taken at 1 lam on 19 March 1987) exhibits the two peaks and a good estimation of the total frequency, $\Delta f=0.620117 \mathrm{~Hz}$, compared to the theoretical one, $\Delta f_{t}=0.620583 \mathrm{~Hz}$, and figure 3.9 (file EB0329.011, measurement taken at 11 am on 29 March 1987) with the same $\Delta f_{t}$ shows what occurs in almost all the cases (only one peak), here at the frequency $f_{e}=0.37 \mathrm{~Hz}$. Taking 
the former $f_{e}$ and half of the theoretical frequency difference, an approximation of the shift frequency, $f_{u}$, can be obtained. Therefore, this technique is of interest in surface current calculations.

However, since it is impossible to get the second-order contribution, we prefer to use the autoregressive spectral estimation.

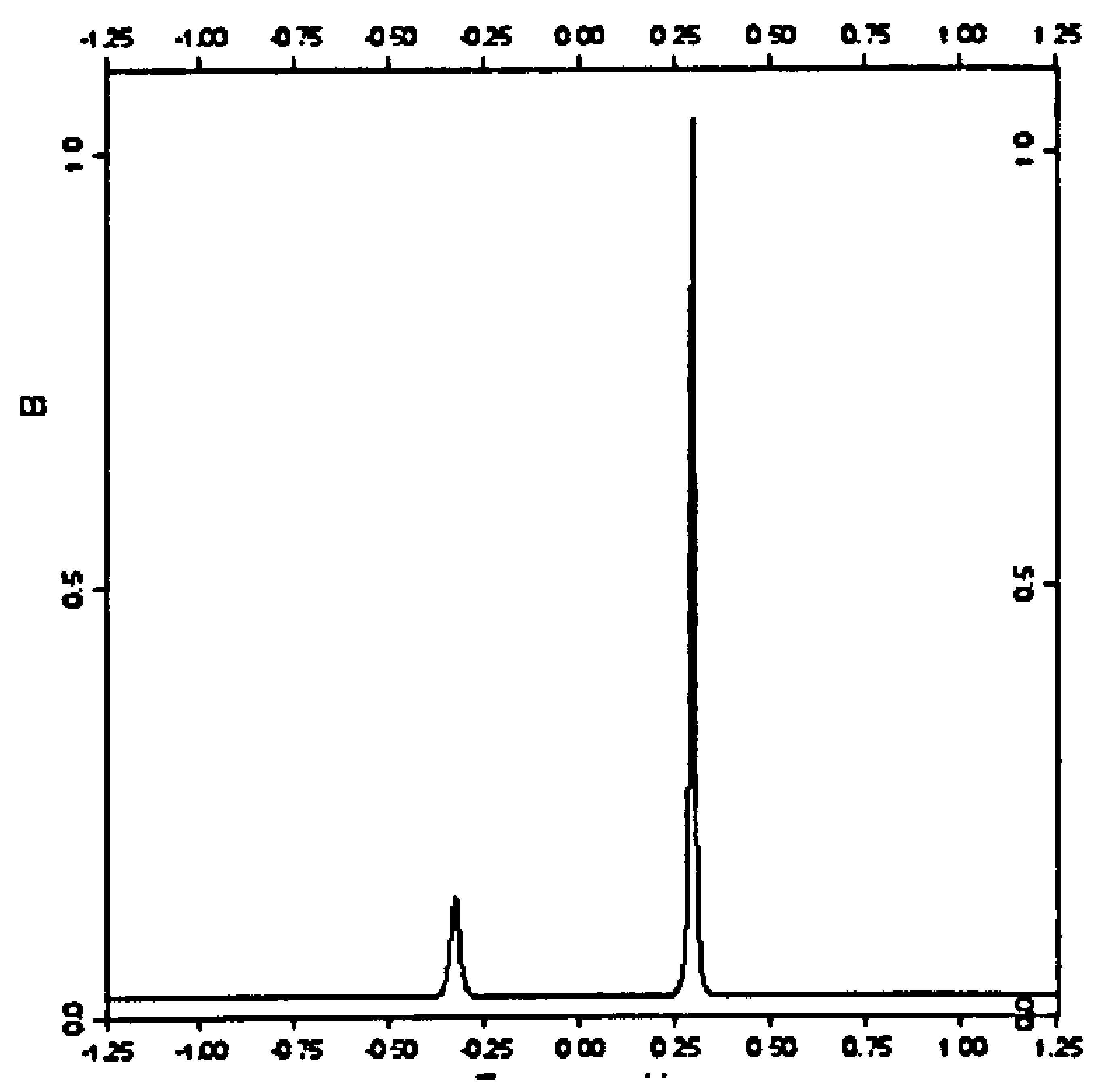

Figure 3.8: MUSIC estimate of the PISCES file EB0319.011 with $N=90$ and $p=2$.

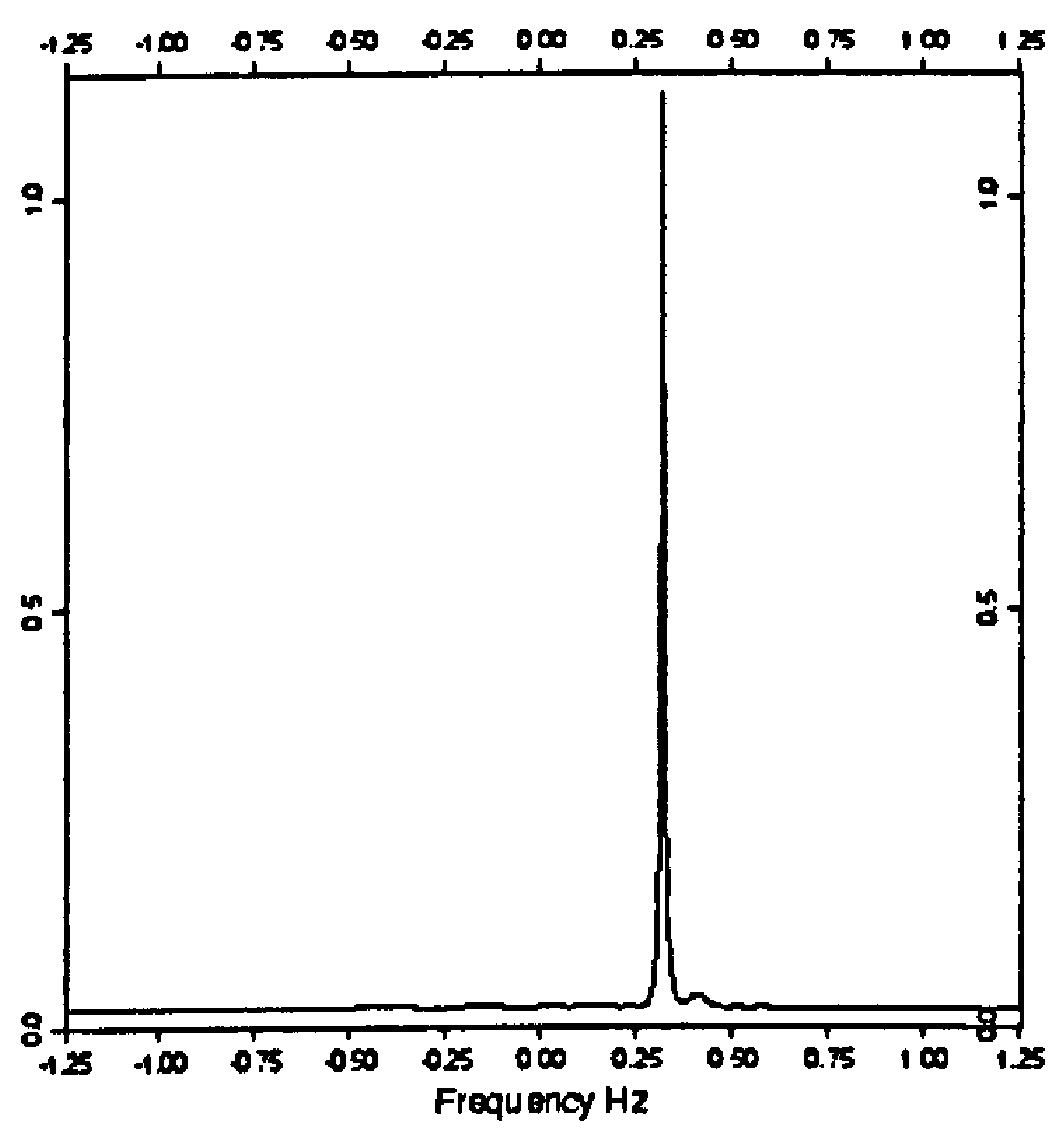

Figure 3.9: MUSIC estimate of the PISCES file EB0329.011 with $N=90$ and $p=2$. 


\subsection{Recursive MLE method}

The Maximum Likelihood Estimation (MLE) method proposed by Kay (1983) develops an estimate of the reflection coefficients, $k_{p}$, and the order update of the autoregressive coefficients, $a_{p}(1), \ldots, a_{p}(p)$, which are provided by the Levinson recursion seen in section 3.1 equation (3.3). A description of this technique is given in Appendix C.

It requires that $X=\{x(1), \ldots, x(N)\}$ be a real Gaussian random process because it rapidly becomes complicated in the complex case : the cubic equation (see Appendix C equation (C.16)) which produces the estimates of reflection coefficients, is too difficult to be solved: the unknown appears as its modulus and as itself.

The probability density function PDF of a real $\operatorname{AR}(p)$ is denoted by:

$$
p\left(X ; a(1), \ldots, a(p), \sigma^{2}\right) .
$$

Let $a=(a(1), . ., a(p))^{T}$. Then

$$
\begin{aligned}
p\left(X ; a(1), . ., a(p), \sigma^{2}\right) & =p\left(x(p), . ., x(N) / x(1), . ., x(p-1) ; a, \sigma^{2}\right) \\
& \times p\left(x(1), \ldots, x(p-1) ; a, \sigma^{2}\right)
\end{aligned}
$$

For large data records (more than 20 samples) which is the case here (100 samples), the maximization of the PDF or likelihood function can be effected approximately by maximizing only the conditional PDF in the right-hand-side of equation (3.21). The effect of the PDF $p\left(x(1), . ., x(p-1) ; a, \sigma^{2}\right)$ on the MLE will be small as long as the poles are not too close to the unit circle (see Kay, 1983). With this approximation the conditional PDF can be maximized by assuming that $X=\{x(1), \ldots, x(N)\}$ is a Gaussian random process. We have shown that this is true for our data (see Chapter 2 section 2.5 ).

The MLE method is used on the real or imaginary parts of the data. Another version of the subroutine TEST of the algorithm is included in Appendix A because of errors in the published code (Kay, 1988, p 267). Determining the root of the cubic equation which is the maximum likelihood estimate of the reflection coefficient, we must check firstly if the parameter $D$ is positive (see Kay, 1983, section III p 58). 
This estimator is a closer approximation to the true MLE than the one obtained by linear prediction methods. Operating in a recursive way, it successively fits higher order AR models to the data (and the estimated all-pole filter is guaranteed to be stable). The RMLE does well with respect to the absence of spurious peaks and resolvability of closely spaced peaks. However, the requirement of real data is too restrictive. This method is not as good as the modified covariance or Burg method for spectral peak location estimations. Using the real part of the data (file EB0319.011), figures 3.10 and 3.11 exhibit respectively the RMLE spectrum and the periodogram, giving the same frequency (around $0.3 \mathrm{~Hz}$ ) for the first-order peak as shown in figure 2.6. They are similar and of course both are symmetric, due to the absence of the imaginary contribution.

Therefore, we prefer to leave this method aside.

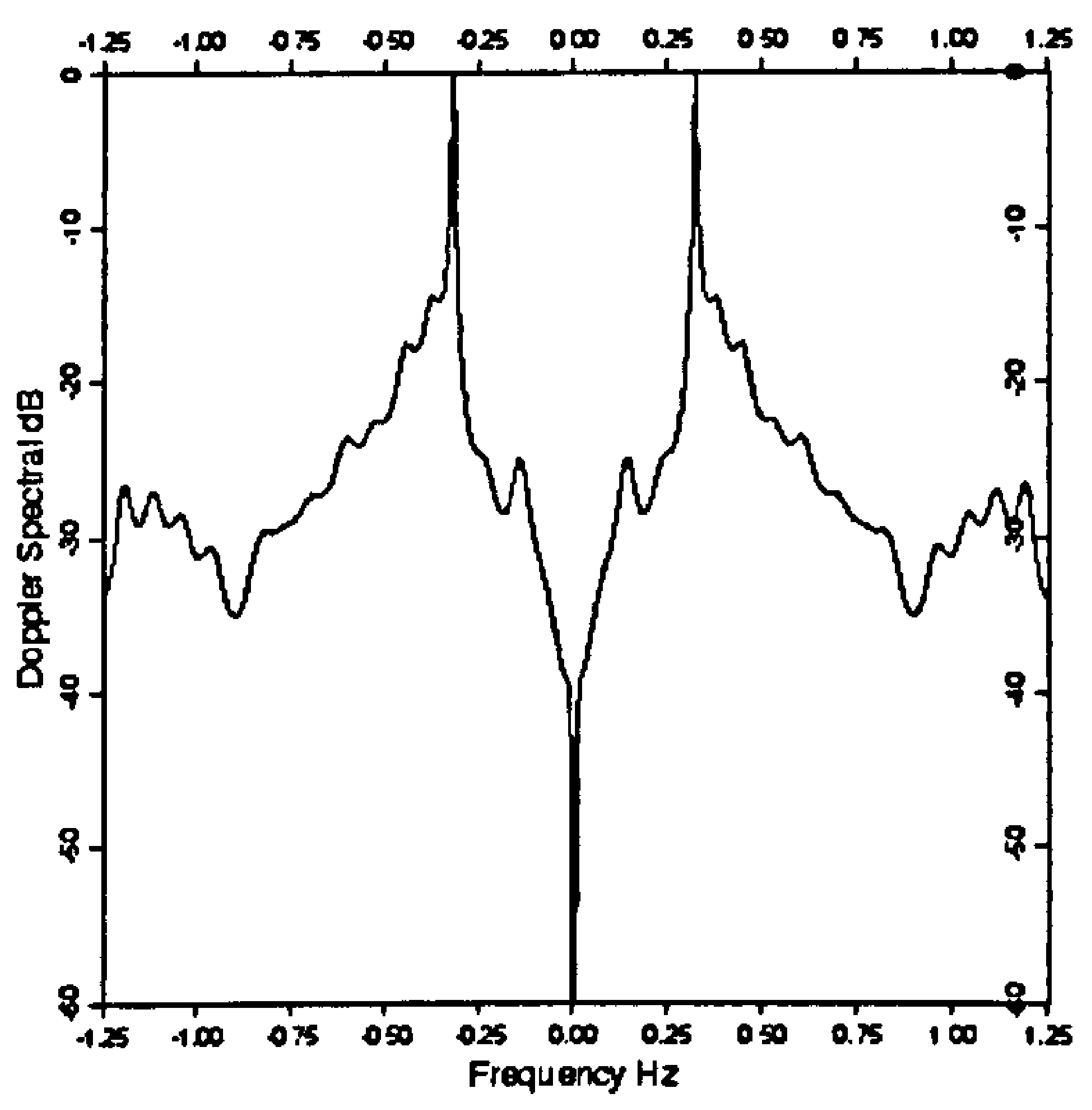

Figure 3.10: RMLE spectrum from the real part of the PISCES file EB0319.011 with $N=100$. 


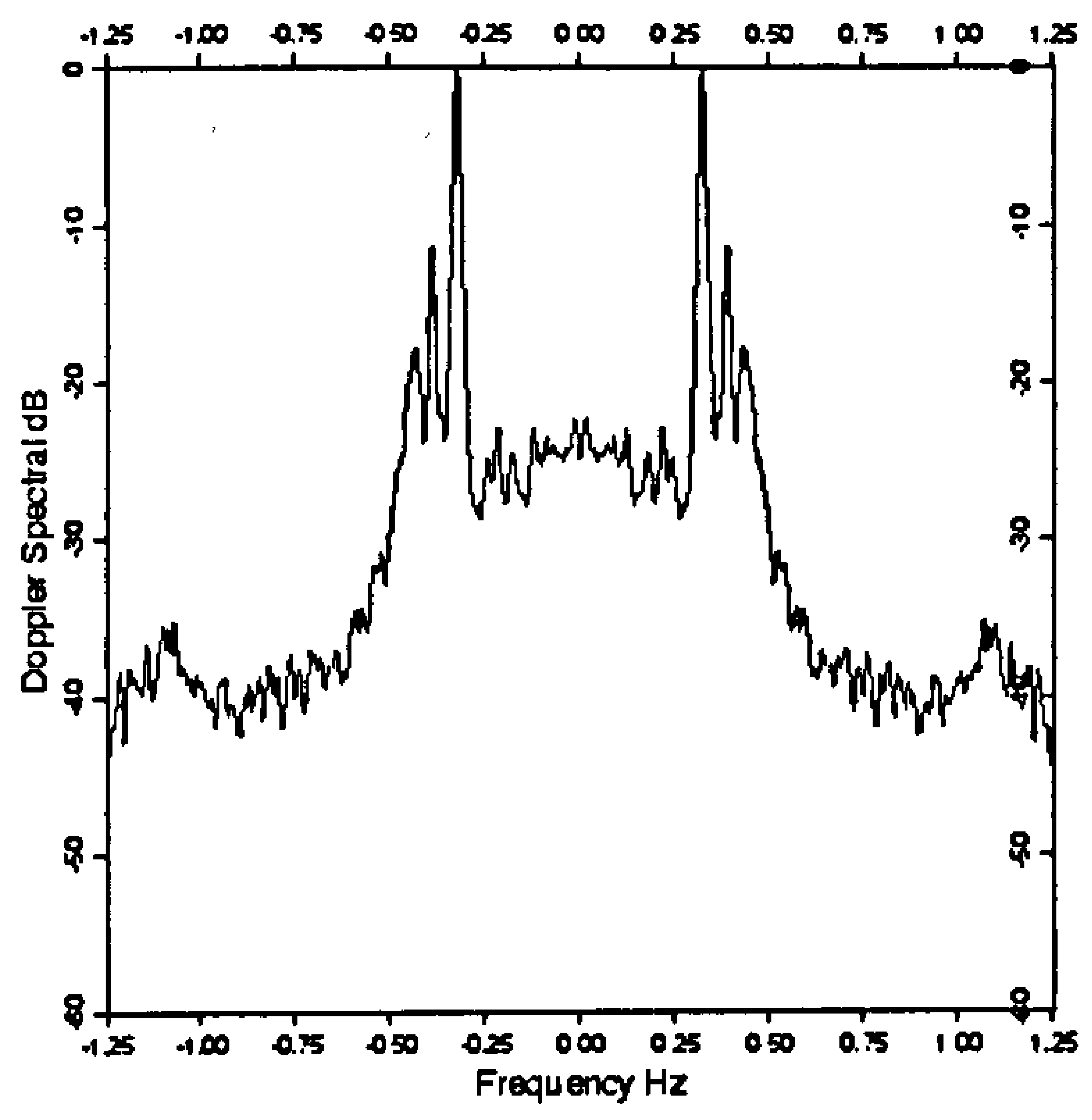

Figure 3.11: Periodogram from the real part of the PISCES file EB0319.011.

\subsection{Summary}

In this chapter, four different modern spectral estimations were considered. We chose to work with the autoregressive spectral estimation and particularly, with the modified covariance method because of its reliability and efficiency concerning the position of the two Bragg peaks. A full description of this technique is given in the next chapter. 


\section{Chapter 4}

\section{The Modified Covariance Method}

In the previous chapter, we presented several modern spectral estimation method. The modified covariance method, introduced by Marple in 1980, was chosen because it revealed to be the most reliable and efficient concerning the first-order part of the spectrum.

A full description of this technique, based on the autoregressive spectral estimation, is given for completeness. It is supplemented by some details not presented elsewhere. Some applications on HF radar data are shown and superposed with the periodogram in order to show the similarities between the second-order contribution.

Based on the autoregressive model, we also point out its major problem which is the selection of the model order.

Finally, we propose this method as a solution to the problem of split peaks mentioned in the Introduction, because of its ability to use short data sets. 


\subsection{Description}

This method performs combined minimization of the forward and backward linear prediction squared errors, and makes estimates of the linear prediction coefficients or AR parameters.

Let $\{x(n)\}$ be the $N$-point data sequence $x(1), \ldots, x(N)$ which estimate the $p$ th-order AR parameters.

Assume that $x(n)=0$ for $n<0$ and $n>N$.

The forward linear predictor $\hat{x}(n)$ for sample $x(n)$ is such that

$$
\hat{x}(n)=-\sum_{k=1}^{P} a_{p}(k) x(n-k)
$$

where the $a_{p}(k)$ are the forward linear prediction coefficients for order $p$.

The forward linear prediction error is

$$
\begin{aligned}
e_{p}^{f}(n) & =x(n)-\hat{x}(n)=x(n)+\sum_{k=1}^{p} a_{p}(k) x(n-k) \\
& =(x(n) \ldots x(n-p))\left(\begin{array}{c}
1 \\
a_{p}(1) \\
\ldots \\
a_{p}(p)
\end{array}\right) \quad \text { for } n=p+1, \ldots, N \\
& =\mathbf{X}_{p}^{T}(n) \mathbf{a}_{p}^{f b}
\end{aligned}
$$

For purposes of fitting an AR model, it is assumed that the prediction error is a whitened process. This equates the AR parameters to the linear prediction coefficients.

Assuming $\{x(n)\}$ to be a stationary random process, the forward and backward linear prediction coefficients are complex conjugates of one another. So the backward linear prediction error may be expressed as

$$
\begin{aligned}
e_{p}^{b}(n) & =x(n-p)+\sum_{k=1}^{p} a_{p}^{*}(k) x(n-p+k) \\
& =\mathbf{X}_{p}^{T}(n) \mathbf{J a}_{p}^{f *^{*}}
\end{aligned}
$$

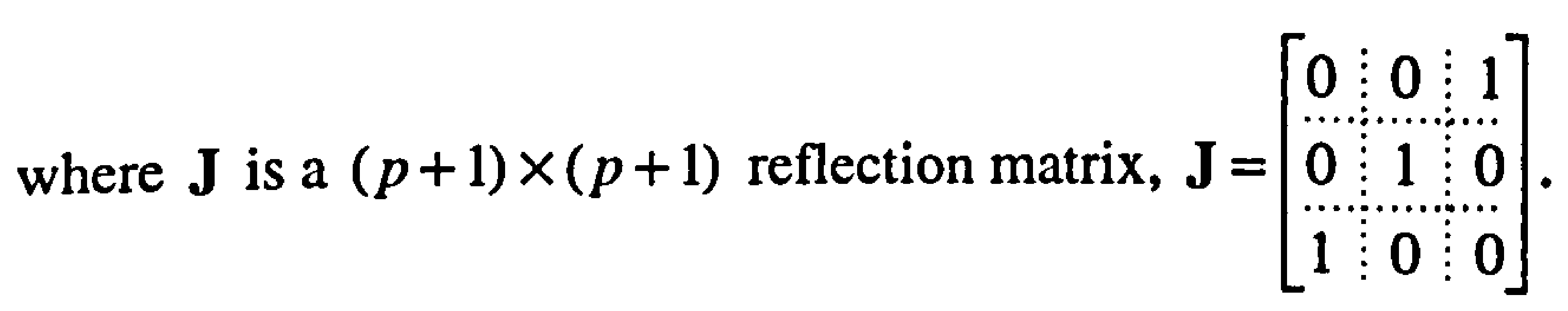


The modified covariance method minimizes the sum of the forward and the backward linear prediction squared errors:

$$
\rho_{p}^{f b}=\sum_{n=p+1}^{N}\left(\left|e_{p}^{f}(n)\right|^{2}+\left|e_{p}^{b}(n)\right|^{2}\right)
$$

This leads to the set of normal equations

$$
\frac{\partial \rho_{p}^{f b}}{\partial a_{p}(i)}=0 \Rightarrow \sum_{j=0}^{p} a_{p}(j) r_{p}(i, j)=0 \text { for } i \neq 0
$$

where $r_{p}(i, j)=\sum_{n=p+1}^{N}\left(x^{*}(n-i) x(n-j)+x(n-p+i) x^{*}(n-p+j)\right)$ and $a_{p}(0)=1$.

Using (4.5), the definitions (4.2) and (4.3) and the fact that $r_{p}(0, j)=r_{p}^{*}(j, 0)$, we find:

$$
\rho_{p}^{f b}=\sum_{j=0}^{p} a_{p}(j) r_{p}(0, j)
$$

Expressions (4.5) and (4.7) can be combined into a single $(p+1) \times(p+1)$ matrix expression:

$$
\mathbf{R}_{p} \mathbf{a}_{p}^{f b}=\left[\begin{array}{c}
\rho_{p}^{f b} \\
0_{p}
\end{array}\right]
$$

with $\mathbf{R}_{p}=\sum_{n=p+1}^{N}\left(\mathbf{X}_{p}^{*}(n) \mathbf{X}_{p}^{T}(n)+\mathbf{J} \mathbf{X}_{p}(n) \mathbf{X}_{p}^{* T}(n) \mathbf{J}\right)$.

So we need to solve these $(p+1)$ equations for the $(p+1)$ unknowns $a_{p}(1), \ldots, a_{p}(p)$ and $\rho_{p}^{f b}$. 


\subsection{Fast algorithm to solve the modified covariance equations of linear prediction}

Using (4.7), we can see that $\mathbf{R}_{p}$ is persymmetric $\left(\mathbf{J R}_{p}^{*} \mathbf{J}=\mathbf{R}_{p}\right.$ ) and also Hermitian $\left(\mathbf{R}_{p}^{* T}=\mathbf{R}_{p}\right)$. These properties allow a recursive algorithm of order $p^{2}$ operations.

If $e_{p}^{f}(p+1)$ and $e_{p}^{b}(N)$ are both not used, the resulting squared error $\rho_{p}^{f b}$ is minimized and the linear prediction coefficients satisfy the normal equations:

$$
\mathbf{R}_{p}^{\prime} \mathbf{a}_{p}^{f b^{\prime}}=\left[\begin{array}{l}
\rho_{p}^{f b^{\prime}} \\
0
\end{array}\right]
$$

with $\rho_{p}^{f b^{\prime}}=\frac{1}{2}\left[\sum_{n=p+2}^{N}\left(\left|e_{p}^{f^{\prime}}(n)\right|^{2}+\left|e_{p}^{b^{\prime}}(n-1)\right|^{2}\right)\right]$.

The prime denotes the solution for the case of omitted error terms and $\rho_{p}^{f b^{\circ}}$ is the time-index-shifted variant of the definition (4.5) for $\rho_{p}^{f b}$. This is independent of time; it is only here to indicate the successive cancellations of the error terms.

These definitions will be useful in the development of the recursive algorithm that follows.

\subsubsection{Special partitions of $R_{p}$}

\subsubsection{Order-index partition}

The properties imply that $\mathbf{R}_{p}$ is $(p+1) \times(p+1)$ matrix such that:

$$
\mathbf{R}_{p}=\left[\begin{array}{cc}
\mathbf{R}_{p-1}^{\prime} & \mathbf{r}_{p} \\
\mathbf{r}_{p}^{H} & r_{p}(p, p)
\end{array}\right]=\left[\begin{array}{cc}
r_{p}(0,0) & \mathbf{r}_{p}^{T} \mathbf{J} \\
\mathbf{J r}_{p}^{*} & \mathbf{J R}_{p-1}^{*} \mathbf{J}
\end{array}\right]
$$

where $\quad \mathbf{r}_{p}=\left[\begin{array}{c}r_{p}(0, p) \\ \ldots \ldots \ldots . . \\ r_{p}(p-1, p)\end{array}\right]$. 


\subsubsection{Time-index partition}

$$
\begin{aligned}
\mathbf{R}_{p} & =\mathbf{R}_{p}^{\cdot}+\mathbf{X}_{p}^{*}(p+1) \mathbf{X}_{p}^{T}(p+1)+\mathbf{J} \mathbf{X}_{p}(N) \mathbf{X}_{p}^{H}(N) \mathbf{J} \\
& =\mathbf{J} \mathbf{R}_{p}^{*} \mathbf{J}+\mathbf{J} \mathbf{X}_{p}(p+1) \mathbf{X}_{p}^{H}(p+1) \mathbf{J}+\mathbf{X}_{p}^{*}(N) \mathbf{X}_{p}^{T}(N)
\end{aligned}
$$

\subsubsection{Auxiliary parameters}

We also need two $(p+1) \times 1$ vectors: $c_{p}$ and $d_{p}$ defined by the matrix equations

$$
\begin{aligned}
\mathbf{R}_{p} \mathbf{c}_{p}=\mathbf{J} \mathbf{X}_{p}(N) \text { and } \mathbf{R}_{p} \mathbf{d}_{p}=\mathbf{X}_{p}^{*}(p+1) \\
\text { with } \mathbf{c}_{p}=\left[\begin{array}{c}
c_{p}(0) \\
\ldots \ldots \ldots . . . \\
c_{p}(p)
\end{array}\right] \text { and } \mathbf{d}_{p}=\left[\begin{array}{c}
d_{p}(0) \\
\ldots \ldots \ldots . . . \\
d_{p}(p)
\end{array}\right]
\end{aligned}
$$

Due to the Hermitian persymmetry of $\mathbf{R}_{p}$, we have

$$
\left\{\begin{array}{c}
\mathbf{J R}_{p}^{*} \mathbf{a}_{p}^{f b^{*}}=\mathbf{R}_{p} \mathbf{J a}_{p}^{f b^{*}}=\left(\begin{array}{c}
0_{p} \\
\rho_{p}^{f b}
\end{array}\right) \\
\mathbf{R}_{p} \mathbf{J c}_{p}^{*}=\mathbf{X}_{p}^{*}(N) \\
\mathbf{R}_{p} \mathbf{J d}_{p}^{*}=\mathbf{J} \mathbf{X}_{p}(p+1)
\end{array}\right.
$$

Using (4.2), vectors $\hat{\mathbf{c}}_{p}^{\prime}$ and $\mathbf{d}_{p}^{\prime}$ are defined for the matrix $R_{p}^{\prime}$ in a similar manner :

$$
\left\{\begin{array}{c}
\left(\mathbf{J R}_{p}^{*} \mathbf{J}\right) \mathbf{c}_{p}^{\prime}=\mathbf{J X} \mathbf{X}_{p}(N) \\
\left(\mathbf{J R}_{p}^{*} \mathbf{J}\right) \mathbf{d}_{p}^{\prime}=\mathbf{X}_{p}^{*}(p+1)
\end{array}\right.
$$

\subsubsection{Order Update Recursions}

Using the order-index partition of $\mathbf{R}_{p}$, we can show that the order update for the linear prediction coefficient vector is

$$
\mathbf{a}_{p}^{f b}=\left[\begin{array}{c}
f b_{p-1}^{\prime} \\
0
\end{array}\right]+a_{p}(p)\left[\begin{array}{c}
0 \\
\mathbf{J a}_{p-1}^{f b^{*}}
\end{array}\right]
$$


where $a_{p}(p)=-\frac{\Delta_{p}}{\rho_{p-1}^{f b^{\prime}}}$ and $\Delta_{p}=\mathbf{r}_{p}^{H} \mathbf{a}_{p-1}^{f b}$.

This is verified by multiplying by $\mathbf{R}_{p}$, as follows:

$$
\begin{gathered}
L H S=\mathbf{R}_{p} \mathbf{a}_{p}^{f b} \\
R H S=\left[\begin{array}{cc}
\mathbf{R}_{p-1}^{\prime} & \mathbf{r}_{p} \\
\mathbf{r}_{p}^{* T} & r_{p}(p, p)
\end{array}\right]\left[\begin{array}{c}
\mathbf{a}_{p-1}^{f b^{\prime}} \\
0
\end{array}\right]+a_{p}(p)\left[\begin{array}{cc}
r_{p}(0,0) & \mathbf{r}_{p}^{T} \mathbf{J} \\
\mathbf{J} \mathbf{r}_{p}^{*} & \mathbf{J R}_{p-1}^{*} \mathbf{J}
\end{array}\right]\left[\begin{array}{c}
0 \\
\mathbf{J} \mathbf{a}_{p-1}^{f b^{*}}
\end{array}\right] \\
L H S=R H S \Rightarrow\left[\begin{array}{c}
\rho_{p}^{f b} \\
\mathbf{0}_{p}
\end{array}\right]=\left[\begin{array}{c}
\mathbf{R}_{p-1}^{\prime} \mathbf{a}_{p-1}^{f b^{\prime}} \\
\mathbf{r}_{p}^{* T} \mathbf{a}_{p-1}^{f b^{\prime}}
\end{array}\right]+a_{p}(p)\left[\begin{array}{c}
\mathbf{r}_{p-1}^{T} \mathbf{a}_{p-1}^{f b^{*}} \\
\mathbf{J} \mathbf{R}_{p-1}^{*} \mathbf{a}_{p-1}^{f b^{* *}}
\end{array}\right] \\
\Rightarrow\left[\begin{array}{c}
\rho_{p}^{f b} \\
\mathbf{0}
\end{array}\right]=\left[\begin{array}{c}
\rho_{p-1}^{f b^{\prime}} \\
\mathbf{0}_{p-1} \\
\Delta_{p}
\end{array}\right]-\left[\begin{array}{c}
\left|\Delta_{p}\right|^{2} \\
\rho_{p-1}^{f b^{\prime}} \\
\mathbf{0}_{p-1} \\
\Delta_{p}
\end{array}\right]
\end{gathered}
$$

This yields that the order update for the linear prediction squared error is

$$
\rho_{p}^{f b}=\rho_{p-1}^{f b}-\frac{\left|\Delta_{p}\right|^{2}}{\rho_{p-1}^{f b^{\prime}}}=\rho_{p-1}^{f b^{\prime}}\left(1-\left|a_{p}(p)\right|^{2}\right)
$$

The order update relationships for $c_{p}$ and $d_{p}$ are given by:

$$
\begin{aligned}
& \mathbf{c}_{p}=\left[\begin{array}{c}
0 \\
\mathbf{c}_{p-1}^{\prime}
\end{array}\right]+c_{p}(0) \mathbf{a}_{p} \quad, \quad c_{p}(0)=\frac{e_{p}^{b}(N)}{\rho_{p}^{f b}} \\
& \mathbf{d}_{p}=\left[\begin{array}{c}
0 \\
\mathbf{d}_{p-1}^{\prime}
\end{array}\right]+d_{p}(0) \mathbf{a}_{p} \quad, \quad d_{p}(0)=\frac{e_{p}^{f^{*}}(p+1)}{\rho_{p}^{f b}}
\end{aligned}
$$

The terms $c_{p}(0)$ and $d_{p}(0)$ are determinated from the following scalar identities:

$$
\begin{gathered}
\mathbf{c}_{p}^{* T} \mathbf{R}_{p} \mathbf{a}_{p}^{f b}=\left(\mathbf{a}_{p}^{f b * T} \mathbf{R}_{p} \mathbf{c}_{p}\right)^{*} \\
\Rightarrow \mathbf{c}_{p}^{* T}\left[\begin{array}{c}
\rho_{p}^{f b} \\
\mathbf{0}_{p}
\end{array}\right]=\left(\mathbf{a}_{p}^{f b * T} \mathbf{J} \mathbf{X}_{p}(N)\right)^{*}=e_{p}^{b^{*}}(N)
\end{gathered}
$$




$$
\Rightarrow c_{p}(0)=\frac{e_{p}^{b}(N)}{\rho_{p}^{f b}}
$$

Similarly, we can prove that $d_{p}(0)=\frac{e_{p}^{f^{*}}(p+1)}{\rho_{p}^{f b}}$.

To prove (4.16), we need only to verify the last $p$ rows, multiplying both sides by $\mathbf{R}_{p}:$

$$
\mathbf{J X}_{p}(N)=\left[\begin{array}{cc}
r_{p}(0,0) & \mathbf{r}_{p}^{T} \mathbf{J} \\
\mathbf{J r}_{p}^{*} & \mathbf{J R}_{p-1}^{*} \mathbf{J}
\end{array}\right]\left[\begin{array}{c}
0 \\
\mathbf{c}_{p-1}^{\prime}
\end{array}\right]+\left[\begin{array}{c}
e_{p}^{b}(N) \\
\mathbf{0}_{p}
\end{array}\right]
$$

But,

$$
\mathbf{J} \mathbf{R}_{p-1}^{*} \mathbf{J c}_{p-1}^{\prime}=\mathbf{J X} \mathbf{X}_{p-1}(N)
$$

So we find the result expected. In the same way, we can prove (4.17).

Three scalar parameters with their corresponding order updates are required by the fast algorithm:

$$
\begin{aligned}
& \left\{\begin{array}{l}
\gamma_{p}=1-\mathbf{X}_{p}^{* T}(N) \mathbf{J c}_{p}=1-\mathbf{X}_{p}^{* T}(N) \mathbf{J R}_{p}^{-1} \mathbf{J} \mathbf{X}_{p}(N) \\
\gamma_{p}=\gamma_{p-1}^{\prime}-c_{p}(0) e_{p}^{b^{*}}(N)=\gamma_{p-1}^{\prime}-\left|e_{p}^{b}(N)\right|^{2} / \rho_{p}^{f b}
\end{array}\right. \\
& \left\{\begin{array}{c}
\delta_{p}=1-\mathbf{X}_{p}^{T}(p+1) \mathbf{d}_{p}=1-\mathbf{X}_{p}^{T}(p+1) \mathbf{R}_{p}^{-1} \mathbf{X}_{p}^{*}(p+1) \\
\delta_{p}=\delta_{p-1}^{\prime}-d_{p}(0) e_{p}^{f}(p+1)=\delta_{p-1}^{\prime}-\left|e_{p}^{f}(p+1)\right|^{2} / \rho_{p}^{f b}
\end{array}\right. \\
& \left\{\begin{array}{c}
\lambda_{p}=\mathbf{X}_{p}^{* T}(N) \mathbf{J d}_{p}, \lambda_{p}^{*}=\mathbf{X}_{p}^{T}(p+1) \mathbf{c}_{p} \\
\lambda_{p}=\lambda_{p-1}^{\prime}+\frac{\left(e_{p}^{f}(p+1) e_{p}^{b}(N)\right)^{*}}{\rho_{p}^{f b}}
\end{array}\right.
\end{aligned}
$$

NB: $\gamma_{p}$ and $\delta_{p}$ are real-valued due to the quadratic product definitions and $\lambda_{p}$ is a complex parameter.

We need only to prove the (4.18) and (4.20) order updates because the relationship (4.19) is similar to (4.18).

For (4.18), we use (4.16) and premultiply by $\mathbf{X}_{p}^{* T}(N) \mathbf{J}$ :

$$
\begin{aligned}
& \mathbf{X}_{p}^{* T}(N) J c_{p}=\mathbf{X}_{p}^{* T}(N) \mathrm{J}\left(\begin{array}{c}
0 \\
\mathbf{c}_{p-1}^{\prime}
\end{array}\right)+\mathbf{c}_{p}(0) \mathbf{X}_{p}^{* T}(N) \mathrm{Ja}_{p} \\
& \Rightarrow 1-\gamma_{p}=1-\gamma_{p-1}^{\prime}+c_{p}(0) e_{p}^{b^{*}}(N)
\end{aligned}
$$

and for (4.12) we premultiply the relationship with $\mathbf{d}_{p}$ by $\mathbf{X}_{p}^{* T}(N) \mathbf{J}$. 
One final order recursion is required for $\mathbf{r}_{p}$, which is obtained from the definition (4.6) of $r_{p}(i, j)$ :

$$
\mathbf{r}_{p}=\left[\begin{array}{c}
r_{p}(0, p) \\
\mathbf{r}_{p-1}-\mathbf{X}(N+1-p) \mathbf{X}_{p-2}^{*}(N)-\mathbf{X}^{*}(p) \mathbf{J} \mathbf{X}_{p-2}(p-2)
\end{array}\right]
$$

\subsubsection{Time Update Recursions}

The relationship for the time-shift update for $a_{p}^{f b^{\prime}}$ is:

$$
\mathbf{a}_{p}^{f b^{\prime}}=\mathbf{a}_{p}^{f b}+\alpha_{1}\left(\begin{array}{c}
0 \\
\mathbf{c}_{p-1}^{\prime}
\end{array}\right)+\beta_{1}\left(\begin{array}{c}
0 \\
\mathbf{d}_{p-1}^{\prime}
\end{array}\right)
$$

where $\left\{\begin{array}{c}\alpha_{1}=\left(e_{p}^{b^{*}}(N) \delta_{p-1}^{\prime}+e_{p}^{f}(p+1) \lambda_{p-1}^{\prime}\right) / D E N_{p-1}^{\prime} \\ \beta_{1}=\left(e_{p}^{f}(p+1) \gamma_{p-1}^{\prime}+e_{p}^{b^{*}}(N) \lambda_{p-1}^{* *}\right) / D E N_{p-1}^{\prime} \\ D E N_{p-1}^{\prime}=\gamma_{p-1}^{\prime} \delta_{p-1}^{\prime}-\left|\lambda_{p-1}^{\prime}\right|^{2}\end{array}\right.$

Premultiplying (4.14) by $\mathbf{R}_{p}^{\prime}$ and substituting the relationship for $\mathbf{R}_{p}{ }^{\prime}$ in the multiplication on the right-hand side yields:

$$
\left(\begin{array}{c}
\rho_{p}^{f b^{\prime}} \\
\mathbf{0}_{p}
\end{array}\right)=\left(\begin{array}{c}
\rho_{p}^{f b} \\
\mathbf{0}_{p}
\end{array}\right)+\alpha_{1} A+\beta_{1} B-e_{p}^{f}(p+1) \mathbf{X}_{p}^{*}(p+1)-e_{p}^{b^{*}}(N) \mathbf{J} \mathbf{X}_{p}(N)
$$

with $\quad A=\left(\mathbf{R}_{p}-\mathbf{X}_{p}^{*}(p+1) \mathbf{X}_{p}^{T}(p+1)-\mathbf{J} \mathbf{X}_{p}(N) \mathbf{X}_{p}^{* T}(N) \mathbf{J}\right)\left(\begin{array}{c}0 \\ \mathbf{c}_{p-1}^{\cdot}\end{array}\right)$

and $\quad B=\left(\mathbf{R}_{p}-\mathbf{X}_{p}^{*}(p+1) \mathbf{X}_{p}^{T}(p+1)-\mathbf{J} \mathbf{X}_{p}(N) \mathbf{X}_{p}^{* T}(N) \mathbf{J}\right)\left(\begin{array}{c}0 \\ \mathbf{d}_{p-1}^{\prime}\end{array}\right)$.

Hence,

$$
\begin{aligned}
& \left(\begin{array}{c}
\rho_{p}^{f b^{\prime}} \\
\mathbf{0}_{p}
\end{array}\right)=\left(\begin{array}{c}
\rho_{p}^{f b} \\
\mathbf{0}_{p}
\end{array}\right)+\left(\alpha_{1} \gamma_{p-1}^{\prime}-\beta_{1} \lambda_{p-1}^{\prime}-e_{p}^{b^{*}}(N)\right) \mathbf{J} \mathbf{X}_{p}(N) \\
& +\left(-\alpha_{1} \lambda_{p-1}^{*}+\beta_{1} \delta_{p-1}^{\prime}-e_{p}^{f}(p+1)\right) \mathbf{X}_{p}^{*}(p+1)-\alpha_{1}\left(\begin{array}{c}
e_{p}^{b}(N) \\
0_{p}
\end{array}\right)-\beta_{1}\left(\begin{array}{c}
e_{p}^{f *}(p+1) \\
\mathbf{0}_{p}
\end{array}\right)
\end{aligned}
$$

However, 


$$
\left\{\begin{array}{c}
\alpha_{1} \gamma_{p-1}^{\prime}-\beta_{1} \lambda_{p-1}^{\prime}=e_{p}^{b^{*}}(N) \\
-\alpha_{1} \lambda_{p-1}^{* *}+\beta_{1} \delta_{p-1}^{\prime}=e_{p}^{f}(p+1)
\end{array}\right.
$$

Therefore,

$$
\begin{gathered}
\rho_{p}^{f b^{\prime}}=\rho_{p}^{f b}-\alpha_{1} e_{p}^{b}(N)-\beta_{1} e_{p}^{f^{*}}(p+1) \\
\Rightarrow \rho_{p}^{f b^{\prime}}=\rho_{p}^{f b}-\frac{\left|e_{p}^{b}(N)\right|^{2} \delta_{p-1}^{\prime}+\left|e_{p}^{f}(p+1)\right|^{2} \gamma_{p-1}^{\prime}+2 \operatorname{Re}\left\{e_{p}^{f}(p+1) e_{p}^{b}(N) \lambda_{p-1}^{\prime}\right\}}{D E N_{p-1}^{\prime}}
\end{gathered}
$$

The auxiliary vectors $\mathbf{c}_{p}$ and $d_{p}$ satisfy the equations:

$$
\begin{aligned}
& c_{p}^{\prime}=c_{p}+\alpha_{2} J_{c_{p}^{*}}^{*}+\beta_{2} J_{d}^{*} \\
& \text { and } d_{p}^{\prime}=d_{p}+\alpha_{3} J_{p}^{*}+\beta_{3} J_{p}^{*}
\end{aligned}
$$

where

$$
\begin{aligned}
& \alpha_{2}=\left(\theta_{p} \lambda_{p}^{*}+\phi_{p} \delta_{p}\right) / D E N_{p} \\
& \beta_{2}=\left(\phi_{p} \lambda_{p}+\theta_{p} \gamma_{p}\right) / D E N_{p} \\
& \alpha_{3}={ }^{\left(\varepsilon_{p} \lambda_{p}^{*}+\theta_{p} \delta_{p}\right) / D E N_{p}} \\
& \beta_{3}=\left(\theta_{p} \lambda_{p}+\varepsilon_{p} \gamma_{p}\right) / D E N_{p} \\
& D E N_{p}=\gamma_{p} \delta_{p}-\left|\lambda_{p}\right|^{2} \\
& \theta_{p}=\mathbf{X}_{p}^{T}(N) \mathbf{d}_{p}=\mathbf{X}_{p}^{* T}(p+1) \mathbf{c}_{p} \\
& \phi_{p}=\mathbf{X}_{p}^{T}(N) \mathbf{c}_{p} \\
& \varepsilon_{p}=\mathbf{X}_{p}^{H}(p+1) \mathbf{J} \mathbf{d}_{p} .
\end{aligned}
$$

To verify (4.24), we premultiply both sides by $\mathbf{J R}_{p}^{*} \mathbf{J}$ and substitute time-index partition (4.10):

$$
\begin{gathered}
\mathrm{JR}_{p}^{*} \mathrm{Jc}_{p}^{*}=\mathrm{JR}_{p}^{*} \mathrm{Jc}_{p}+\alpha_{2} \mathrm{JR}_{p}^{*} \mathrm{JJc}_{p}^{*}+\beta_{2} \mathrm{JR}_{p}^{*} \mathrm{JJd}_{p}^{*} \\
\Rightarrow \mathrm{JX} \mathbf{X}_{p}(N)=\mathrm{A}+\mathrm{B}+\mathrm{C}
\end{gathered}
$$

where

$$
\begin{aligned}
\mathbf{A} & =\left(\mathbf{R}_{p}-\mathbf{J} \mathbf{X}_{p}(p+1) \mathbf{X}_{p}^{* T}(p+1) \mathbf{J}-\mathbf{X}_{p}^{*}(N) \mathbf{X}_{p}^{T}(N)\right) \boldsymbol{c}_{p} \\
& =\mathbf{J} \mathbf{X}_{p}(N)-\mathbf{J} \mathbf{X}_{p}(p+1) \theta_{p}-\mathbf{X}_{p}^{*}(N) \phi_{p} \\
\mathbf{B} & =\alpha_{2} \mathbf{X}_{p}^{*}(N)-\alpha_{2} \mathbf{J} \mathbf{X}_{p}(p+1) \lambda_{p}-\alpha_{2} \mathbf{X}_{p}^{*}(N)\left(1-\gamma_{p}\right)
\end{aligned}
$$




$$
\begin{aligned}
& \text { and } \mathbf{C}=\beta_{2} \mathbf{J} \mathbf{X}_{p}(p+1)-\beta_{2} \mathbf{J} \mathbf{X}_{p}(p+1)\left(1-\delta_{p}\right)-\beta_{2} \mathbf{X}_{p}^{*}(N) \lambda_{p}^{*} \\
& \Rightarrow \mathbf{J} \mathbf{X}_{p}(p+1)\left\{\theta_{p}+\alpha_{2} \lambda_{p}-\beta_{2} \delta_{p}\right\}+\mathbf{X}_{p}^{*}(N)\left\{\phi_{p}-\alpha_{2} \gamma_{p}+\beta_{2} \lambda_{p}^{*}\right\}=0
\end{aligned}
$$

Also, we have

$$
\left\{\begin{array}{c}
\theta_{p}+\alpha_{2} \lambda_{p}-\beta_{2} \delta_{p}=\theta_{p}+\frac{1}{D E N_{p}}\left(\theta_{p} \lambda_{p}^{*} \lambda_{p}+\phi_{p} \delta_{p} \lambda_{p}-\phi_{p} \delta_{p} \lambda_{p}-\theta_{p} \gamma_{p} \delta_{p}\right)=\theta_{p}-\theta_{p}=0 \\
\phi_{p}-\alpha_{2} \gamma_{p}+\beta_{2} \lambda_{p}^{*}=\phi_{p}-\frac{1}{D E N_{p}}\left(\theta_{p} \lambda_{p}^{*} \gamma_{p}+\phi_{p} \delta_{p} \gamma_{p}-\phi_{p} \lambda_{p} \lambda_{p}^{*}-\theta_{p} \gamma_{p} \lambda_{p}^{*}\right)=0 .
\end{array}\right.
$$

Thus, similarly for $\mathbf{d}_{p}$, we obtain the expected results.

Finally, the recursions for $\gamma_{p}, \delta_{p}$ and $\lambda_{p}$ are:

$$
\begin{gathered}
\gamma_{p}^{\prime}=\gamma_{p}-\frac{\left(\left|\phi_{p}\right|^{2} \delta_{p}+\left|\theta_{p}\right|^{2} \gamma_{p}+2 \operatorname{Re}\left[\phi_{p} \lambda_{p} \theta_{p}^{*}\right]\right)}{D E N_{p}} \\
\delta_{p}^{\prime}=\delta_{p}-\frac{\left(\left|\theta_{p}\right|^{2} \delta_{p}+\left|\varepsilon_{p}\right|^{2} \gamma_{p}+2 \operatorname{Re}\left[\theta_{p} \lambda_{p} \varepsilon_{p}^{*}\right]\right)}{D E N_{p}} \\
\lambda_{p}^{\prime}=\lambda_{p}+\alpha_{3} \phi_{p}+\beta_{3} \theta_{p}^{*}
\end{gathered}
$$

They are obtained successively by forming $\mathbf{X}_{p}^{* T}(N) \mathbf{J c}_{p}^{\cdot}$ from (4.26), $\mathbf{X}_{p}^{T}(p+1) \mathbf{d}_{p}^{\prime}$ from (4.27) and $\mathbf{X}_{p}^{* T}(N) \mathbf{J d}_{p}^{\prime}$ from (4.28).

\subsubsection{Modified Covariance Algorithm}

For a $p$ th-order model and $N$ data samples, this algorithm requires $N p+6 p^{2}$ computational operations (additions and multiplications) and element storage of $N+4 p$.

For a given value of $N$, the modified covariance method is comparable with the FFT method which requires $N \log N$ operations. In fact, shorter data sets are used with the modified covariance method, which implies less computation. Thus, the modified covariance method is faster than the FFT technique in this case. 
In the algorithm, we check the numerical ill-conditioning or singularity of the normal equations. The squared prediction errors are, by definition, positive scalars and if these become negative, it is due to numerical ill-conditioning. Or if any become zero, it would indicate a singular normal equation matrix. The scalars $\delta$ and $\gamma$ must be bounded between zero and unity (Marple, 1987). If any of these scalars are outside this range, it shows numerical ill-conditioning.

\subsubsection{Algorithm}

\section{Initial conditions:}

$$
\begin{aligned}
& p=0 \\
& r_{0}(0,0)=2 \sum_{n=1}^{N}|x(n)|^{2} \\
& \rho_{0}^{f b^{\prime}}=r_{0}(0,0)-|x(1)|^{2}-|x(N)|^{2} \\
& c_{0}(0)=\frac{x(N)}{r_{0}(0,0)} \\
& d_{0}(0)=\frac{x^{*}(1)}{r_{0}(0,0)} \\
& \lambda_{0}=x^{*}(1) x^{*}(N) / r_{0}(0,0) \\
& \delta_{0}=1-|x(1)|^{2} / r_{0}(0,0) \\
& \gamma_{0}=1-|x(n)|^{2} / r_{0}(0,0) \\
& D E N_{0}=\gamma_{0} \delta_{0}-\left|\lambda_{0}\right|^{2} \\
& \rho_{0}^{f b}=\rho_{0}^{f b} * D E N_{0} \\
& c_{0}^{\prime}(0)=\frac{x^{*}(1)}{\rho_{0}^{f b^{\prime}}} \\
& d_{0}^{\prime}(0)=\frac{x(n)}{\rho_{0}^{f b^{\prime}}}
\end{aligned}
$$




$$
\begin{aligned}
& r_{1}(0,0)=2 \sum_{k=1}^{N-1} x(k+1) x^{*}(k) \\
& a_{1}^{f b}(1)=-\frac{r_{1}(0,0)}{\rho_{0}^{f b}} \\
& \rho_{1}^{f b}=\rho_{0}^{f b}\left(1-\left|a_{1}^{f b}(1)\right|^{2}\right)
\end{aligned}
$$

\section{Main Loop:}

\section{While $p<P$ do:}

- Auxiliary vector and scalar updates:

$$
\begin{aligned}
& c_{p}=\left[\begin{array}{c}
0 \\
c_{p-1}^{\prime}
\end{array}\right]+c_{p}(0) a_{p}(4.16) \\
& d_{p}=\left[\begin{array}{c}
0 \\
d_{p-1}^{\prime}
\end{array}\right]+d_{p}(0) a_{p} \quad(4.17) \\
& \gamma_{p}=\gamma_{p-1}^{\prime}-\frac{\left|e_{p}^{b}(N)\right|^{2}}{\rho_{p}^{f b}} \quad(>0 \& \leq 1) \quad(4.18) \\
& \delta_{p}=\delta_{p-1}^{\prime}-\frac{\left|e_{p}^{f}(p+1)\right|^{2}}{\rho_{p}^{f b}} \quad(>0 \quad \& \leq 1) \quad(4.19) \\
& \lambda_{p}=\lambda_{p-1}^{\prime}-\frac{\left(e_{p}^{f}(p+1) e_{p}^{b}(N)\right)^{*}}{\rho_{p}^{f b}} \quad(4.20) \\
& D E N_{p}=\gamma_{p} \delta_{p}-\left|\lambda_{p}\right|^{2}
\end{aligned}
$$

- Time shift update:

$$
\begin{aligned}
& \rho_{p}^{f^{\prime}}=\rho_{p}^{f b}-\frac{\left|e_{p}^{b}(N)\right|^{2} \delta_{p-1}^{\prime}+\left|e_{p}^{f}(p+1)\right|^{2} \gamma_{p-1}^{\prime}+2 \operatorname{Re}\left\{e_{p}^{f}(p+1) e_{p}^{b}(N) \lambda_{p-1}^{\prime}\right\}}{D E N_{p-1}^{\prime}} \\
& (>0 \& \leq 1)(4.21) \\
& \mathbf{a}_{p}^{f b^{\prime}}=\mathbf{a}_{p}^{f b}+\alpha_{1}\left(\begin{array}{c}
0 \\
c_{p-1}^{\prime}
\end{array}\right)+\beta_{1}\left(\begin{array}{c}
0 \\
d_{p-1}^{\prime}
\end{array}\right)
\end{aligned}
$$




$$
\begin{aligned}
& \dot{c_{p}^{\prime}}=\mathbf{c}_{p}+\alpha_{2} \mathrm{Jc}_{p}^{*}+\beta_{2} \mathrm{Jd}_{p}^{*} \\
& \mathbf{d}_{p}^{\prime}=\mathbf{d}_{p}+\alpha_{3} \mathrm{Jc}_{p}^{*}+\beta_{3} \mathrm{Jd}_{p}^{*} \quad(4.24) \&(4.25) \\
& \gamma_{p}^{\prime}=\gamma_{p}-\frac{\left|\phi_{p}\right|^{2} \delta_{p}+\left|\theta_{p}\right|^{2} \gamma_{p}+2 \operatorname{Re}\left[\phi_{p} \lambda_{p} \theta_{p}^{*}\right]}{D E N_{p}} \quad(>0 \& \leq 1) \\
& \delta_{p}^{\prime}=\delta_{p}-\frac{\left|\theta_{p}\right|^{2} \delta_{p}+\left|\varepsilon_{p}\right|^{2} \gamma_{p}+2 \operatorname{Re}\left[\theta_{p} \lambda_{p} \varepsilon_{p}^{*}\right]}{D E N_{p}} \quad(>0 \& \leq 1) \\
& \lambda_{p}^{*}=\lambda_{p}+\alpha_{3} \phi_{p}^{*}+\beta_{3} \theta_{p}^{*}
\end{aligned}
$$

$p=p+1$

- Order update:

$\mathbf{r}_{p}=\left[\begin{array}{c}r_{p}(0, p) \\ \mathbf{r}_{p-1}-\mathbf{X}(N+1-p) \mathbf{X}_{p-2}^{*}(N)-\mathbf{X}^{*}(p) \mathbf{J} \mathbf{X}_{p-2}(p-2)\end{array}\right]$

$\Delta_{p}=\mathbf{r}_{p}^{H} \mathbf{a}_{p-1}^{f b^{\prime}}$

$a_{p}(p)=-\frac{\Delta_{p}}{\rho_{p-1}^{p^{\prime}}}$

$\mathbf{a}_{p}^{f b}=\left[\begin{array}{c}\mathbf{a}_{p-1}^{f b^{\prime}} \\ 0\end{array}\right]+a_{p}(p)\left[\begin{array}{c}0 \\ \mathbf{J a}_{p-1}^{f b *}\end{array}\right]$

$\rho_{p}^{f b}=\rho_{p-1}^{f b^{\prime}}\left(1-\left|a_{p}(p)\right|^{2}\right) \quad(>0 \& \leq 1)$

\section{END}




\subsection{Model order selection}

The major problem of the modified covariance method is the selection of the AR model order selection, $p$.

Marple (1987, p229-231) proposed many methods according to $N$. But, in general, insufficiently high values of $p$ are obtained, resulting in a very smoothed spectral estimate. On the other hand, too high orders introduce spurious detail.

Lang and McClellan (1980) showed that for the modified covariance method, $p$ must be taken between $N / 3$ and $N / 2$. But we find that it works only when $N \leq 100$; that is not our case because if we take $N \leq 100$, we do not get the second-order detail that we need.

Therefore, we need to use another way for determining $p$. For the OSCR system, this problem is solved in the next chapter (section 5.1.4) which deals with the methodology to compare the modified covariance method with the periodogram. A range for $p$ is given in Chapter 6 section 6.1.3. But, this selection of $p$ stays problematic for the PISCES system (see Chapter 5 section 5.1.1).

\subsection{Applications}

Given that the second-order contribution is of greatest interest, the periodogram and the spectral estimates obtained from the modified covariance method are scaled to the integral under the first-order peak (Wyatt, 1990). This is essential for the inversion procedure to be done and for determining the significant wave height $H_{S}$ and mean period $T_{\omega}$ (see Chapter 2 section 2.4). 


\subsubsection{OSCR system}

This system supplies files with the same theoretical frequency difference, $\Delta f_{t}=1.028361 \mathrm{~Hz}$. The periodogram uses the total data set, $3 \times 896$ samples. We have been working with the master and slave files of the Holderness coastline data set (from the end of 1995 to the beginning of 1996). After a subjective selection of good cells, the modified covariance method was tested with $N=896$. Figures 4.1 and 4.2 show the periodograms of the cell 55 (measurements taken at $2 \mathrm{pm}$ on 21 December 1995 and at 10pm on 9 January 1996) and the corresponding AR PSD estimates with $p=40$ and 30 respectively. These spectral estimates give the same total frequency difference, $\Delta f=1.027189 \mathrm{~Hz}$ and appear similar.

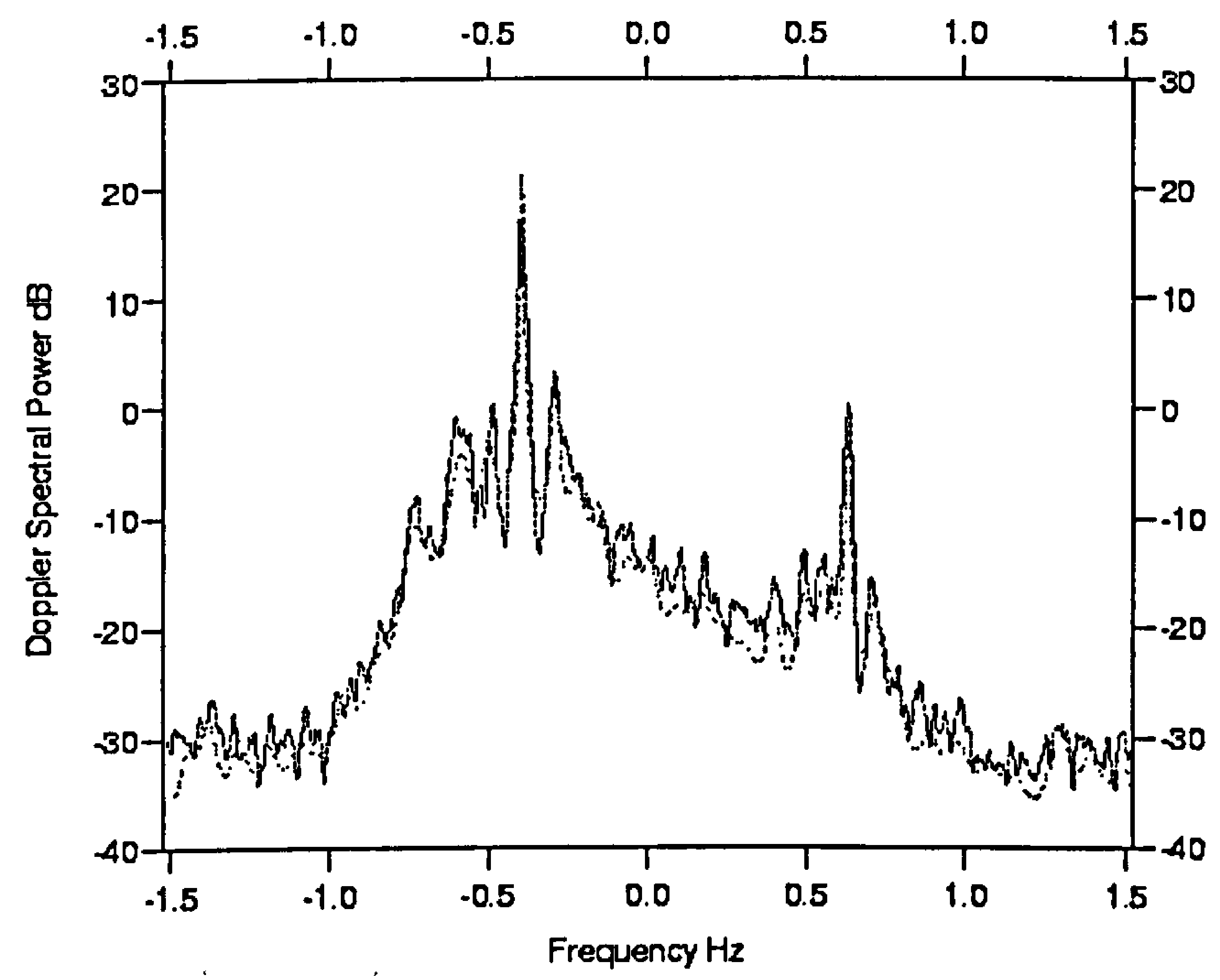

Figure 4.1: Superposition of the periodogram (solid line) and the AR PSD estimate of the OSCR file (dotted line). Measurement taken at 2pm on 21 December 1995 (cell 55). 


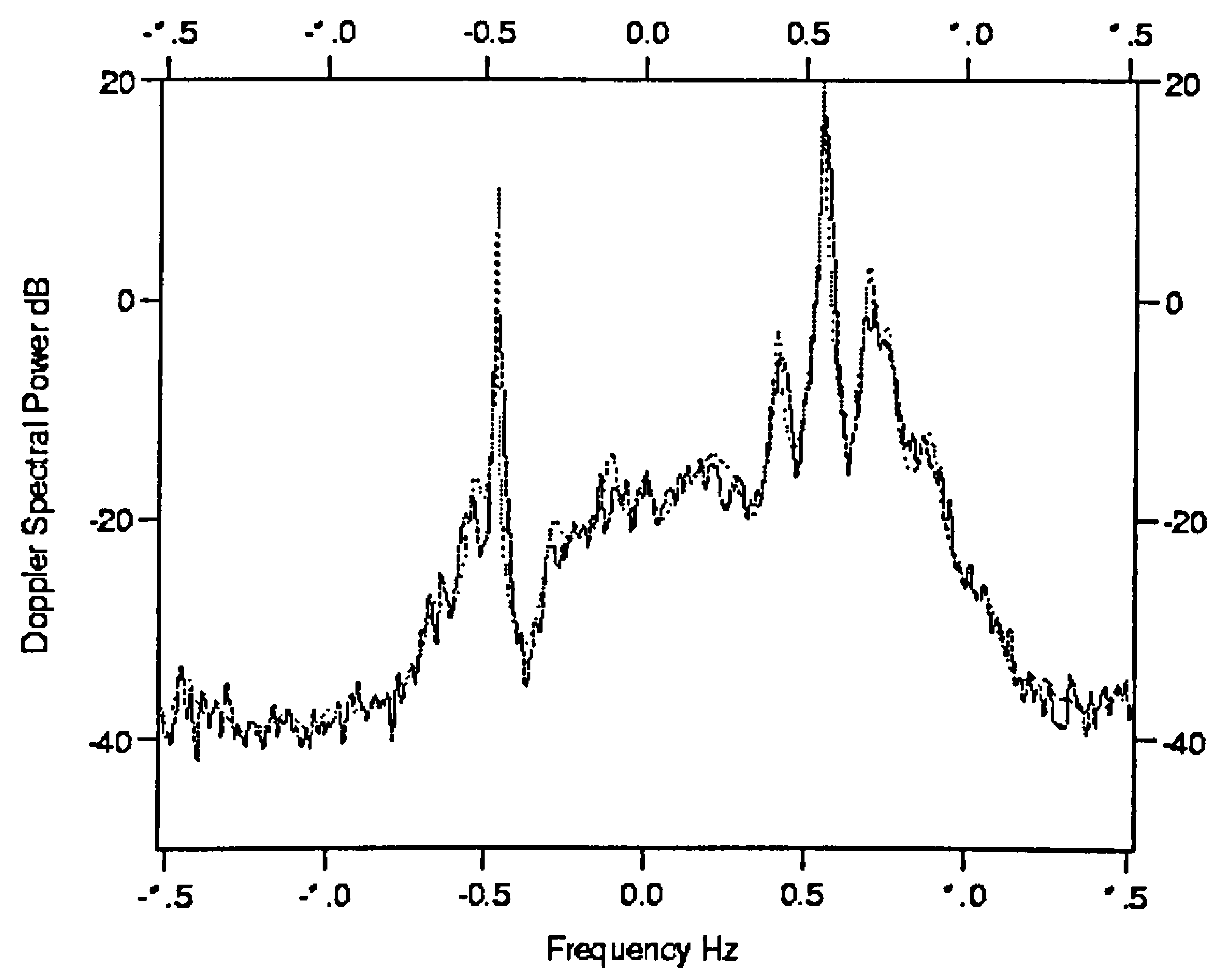

Figure 4.2: Superposition of the periodogram (solid line) and the AR PSD estimate of the OSCR file (dotted line). Measurement taken at 10pm on 9 January 1996 (cell 55).

\subsubsection{PISCES system}

In the previous chapter, the different modern spectral techniques were tested with some data sets of only $N=100$ samples. The objective was to select the most reliable method knowing their crucial aspect of using short data sets.

Figure 4.3 shows an AR PSD estimate with $N=500$ and $p=20$ of the same file used to produce the periodogram shown in figure 2.6. The total frequency difference estimation is $\Delta f=0.620117 \mathrm{~Hz}$, which is close to the theoretical one, $\Delta f_{t}=0.620583 \mathrm{~Hz}$. Note that this AR PSD estimate is "smoother" than the periodogram. This feature of smoothness reveals that $N_{\min }$ is too low if we are considering the second-order contribution or that the model order, $p$, is too small. 


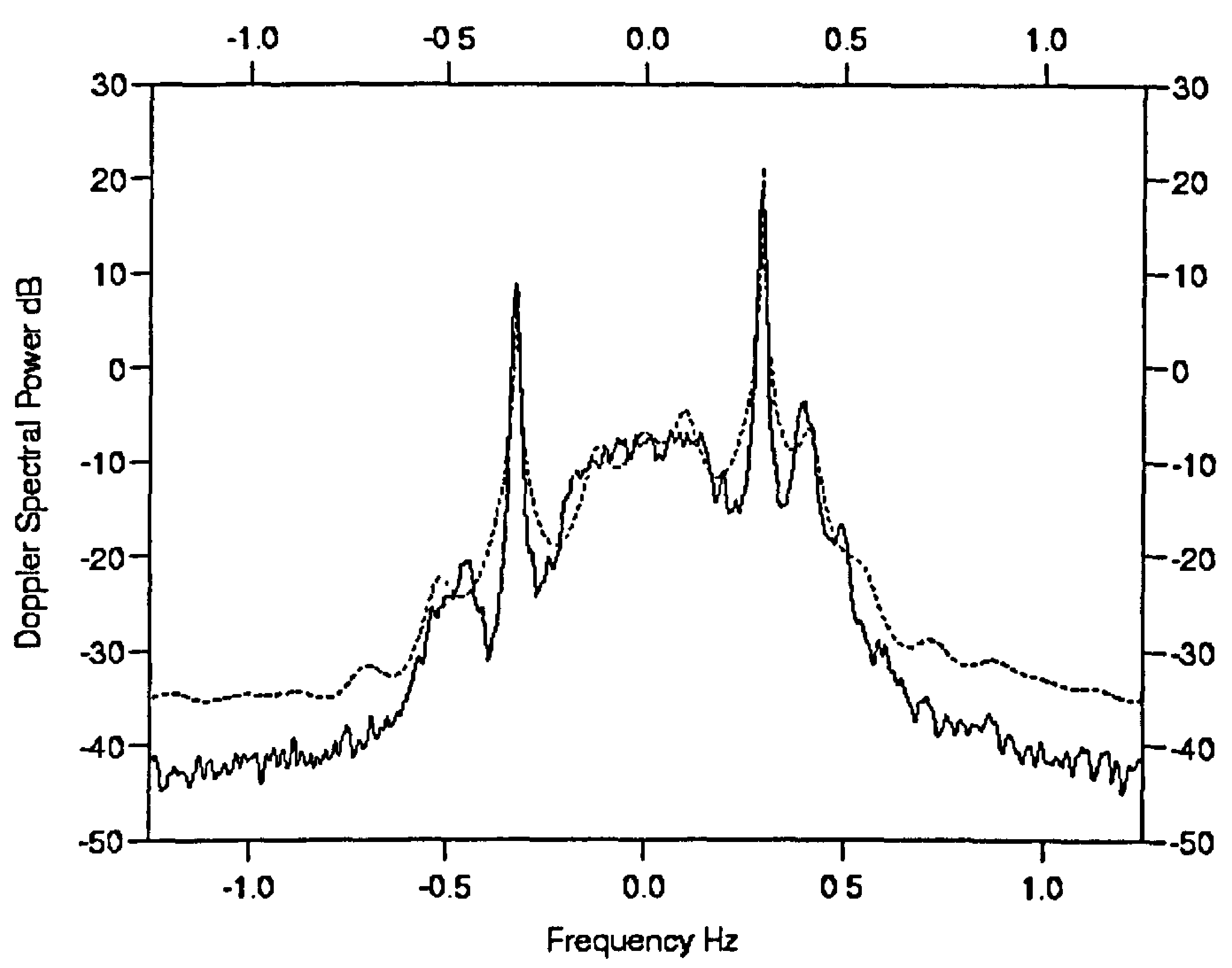

Figure 4.3 : Superposition of the periodogram (solid line) and the AR PSD estimate (dotted line) with $N=500$ and $p=20$ of the PISCES file EB0319.011.

Figure 4.4 exhibits the periodogram and the AR PSD estimate $(N=500$ and $p=20)$ of the file EB0328.008. It can be seen that the second-order contribution near the strongest peak (positive frequency) is not sufficient. Increasing the model order $p$ (see figures 4.5 and 4.6 with $p=50$ and 70 ), improves the continuum surrounding the two main peaks. However, the difference in amplitude of the two main peaks, used to determine the wind direction (see Chapter 2 section 2.3), remains the same (about $23 \mathrm{~dB}$ ) which is larger than for the periodogram (about 10dB). Thus we need more data to apply the modified covariance method. 


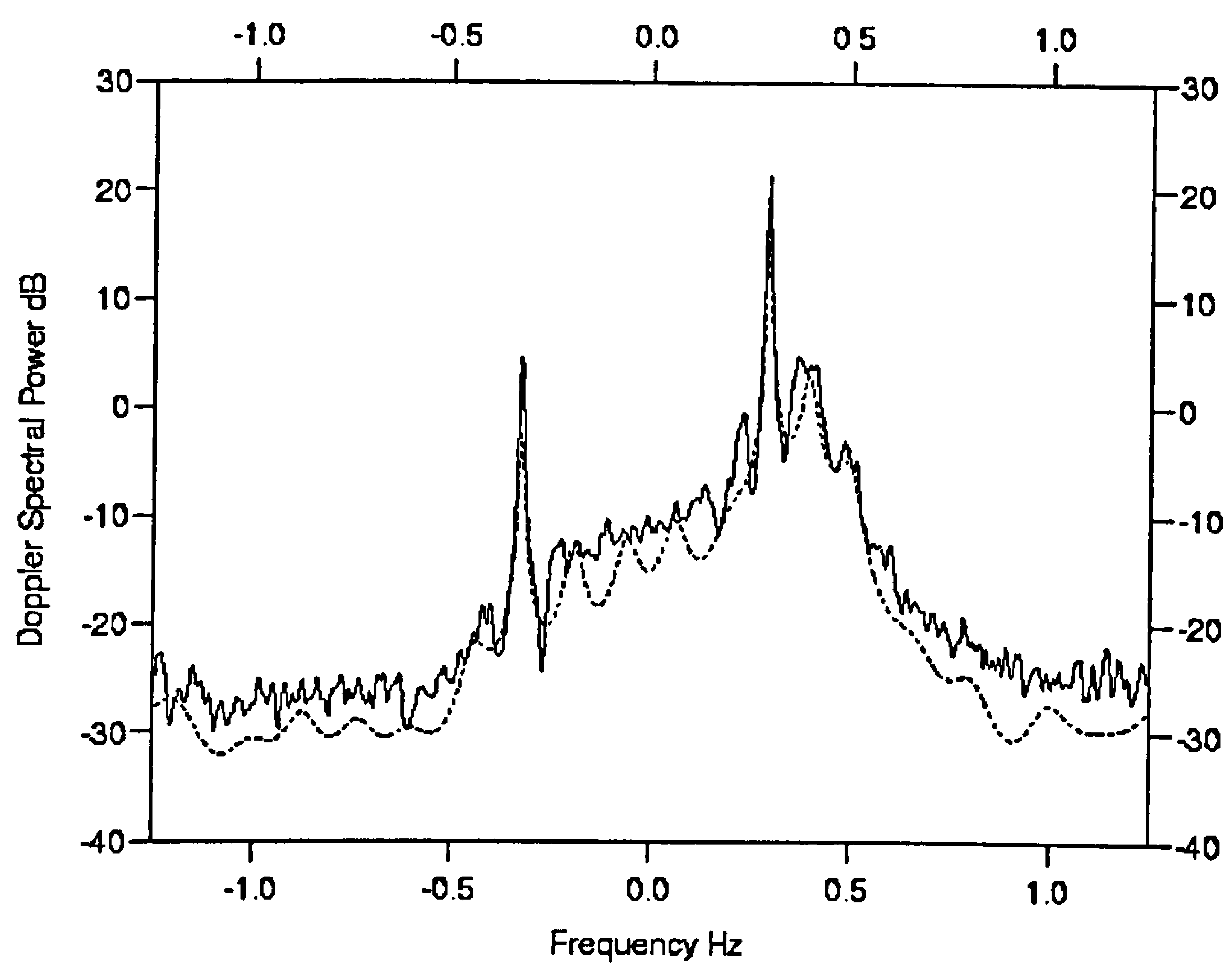

Figure 4.4: Superposition of the periodogram (solid line) and the AR PSD estimate (dotted line) with $N=500$ and $p=20$ of the PISCES file EB0328.008.

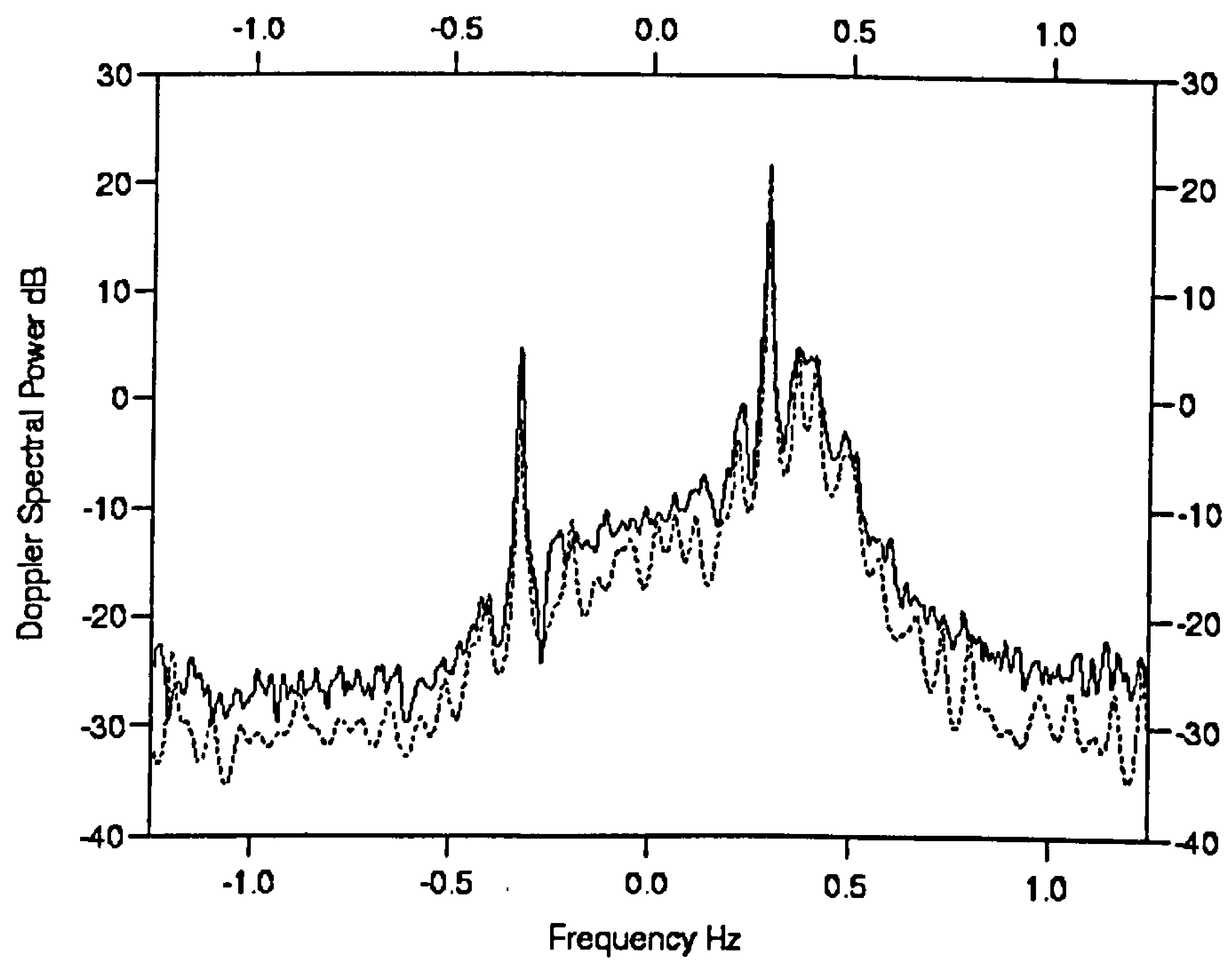

Figure 4.5: Superposition of the periodogram (solid line) and the AR PSD estimate (dotted line) with $N=500$ and $p=50$ of the PISCES file EB0328.008. 


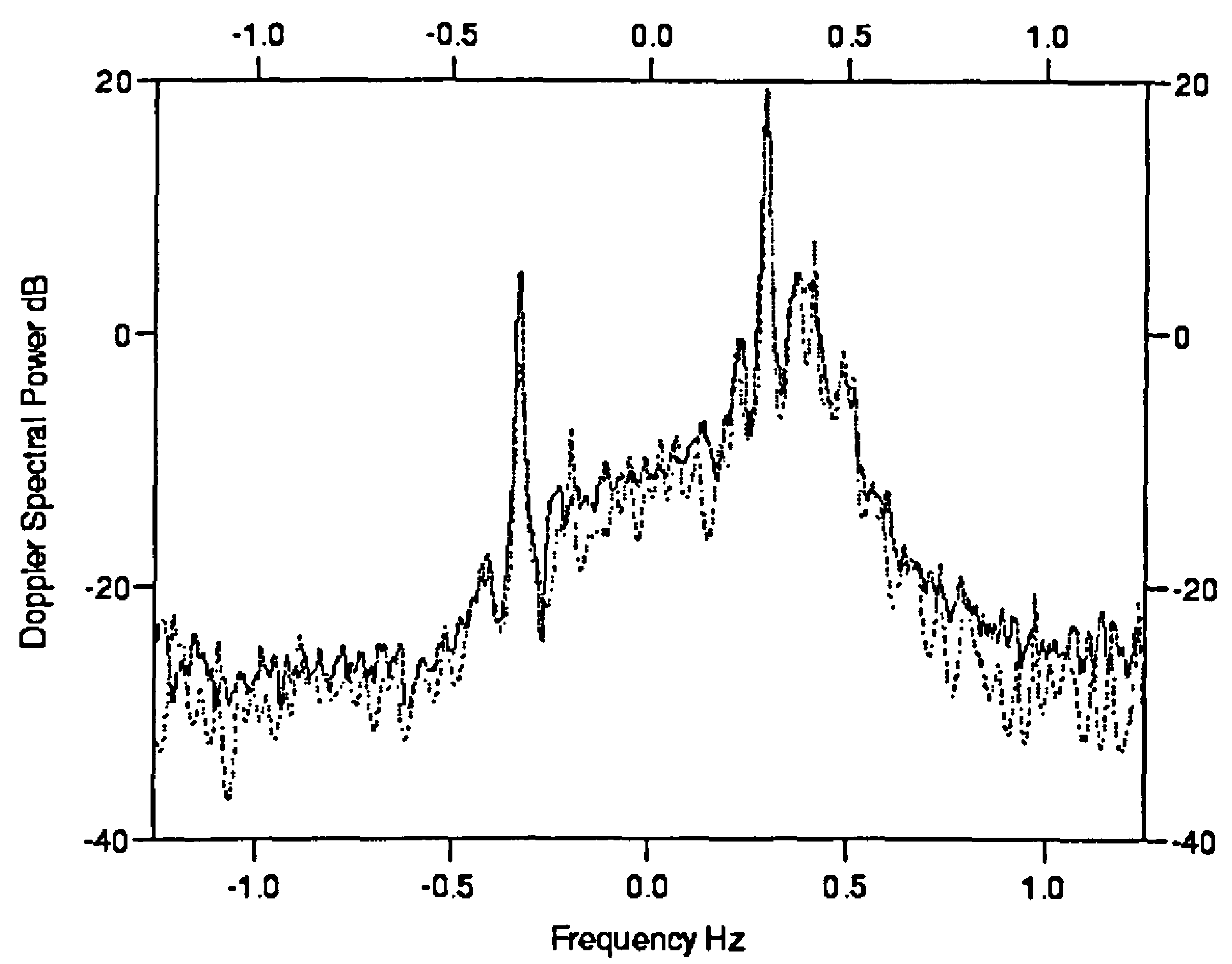

Figure 4.6: Superposition of the periodogram (solid line) and the AR PSD estimate (dotted line) with $N=500$ and $p=70$ of the PISCES file EB0328.008.

To find the minimum data set length $N$ for second-order structure, more tests are needed.

In contrary to the OSCR system which forces us to test the modified covariance method with a maximum of 896 data points, with the PISCES system we can use up to 4608 data points and hence we will be able to find a value of $N$ in order to obtain the best second-order spectrum. This will be presented in Chapter 7.

To show that the modified covariance method does work well, we take all the data, i.e. $N=4608$.

Figures 4.7 and 4.8 exhibit respectively AR PSD estimates of the files EB0319.011 and EB0328.008. They are superposed with their corresponding periodograms. As can be seen, the similarity is striking. 


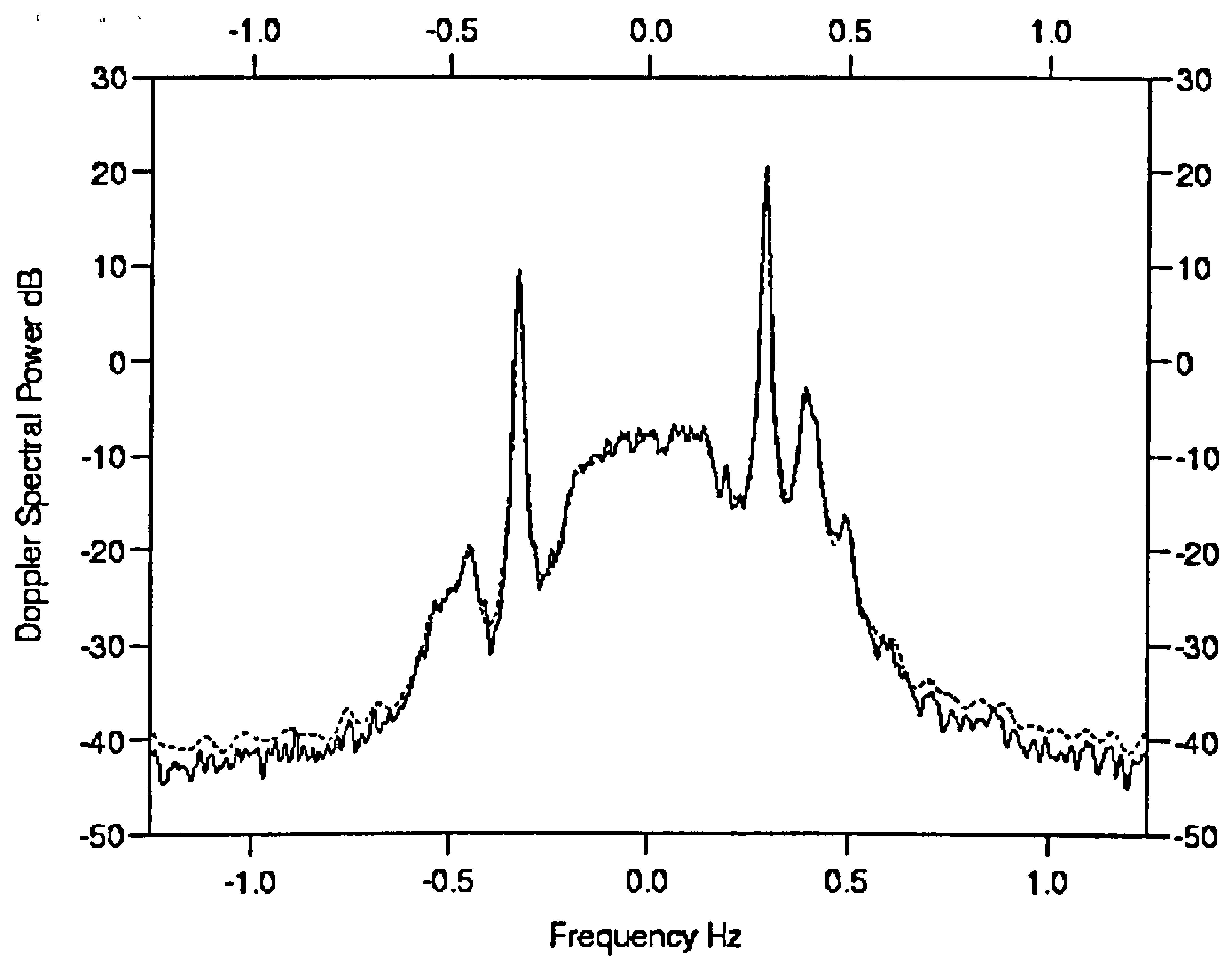

Figure 4.7: Superposition of the periodogram (solid line) and the AR PSD estimate (dotted line) with $N=4608$ and $p=40$, obtained from the PISCES file EB0319.011.

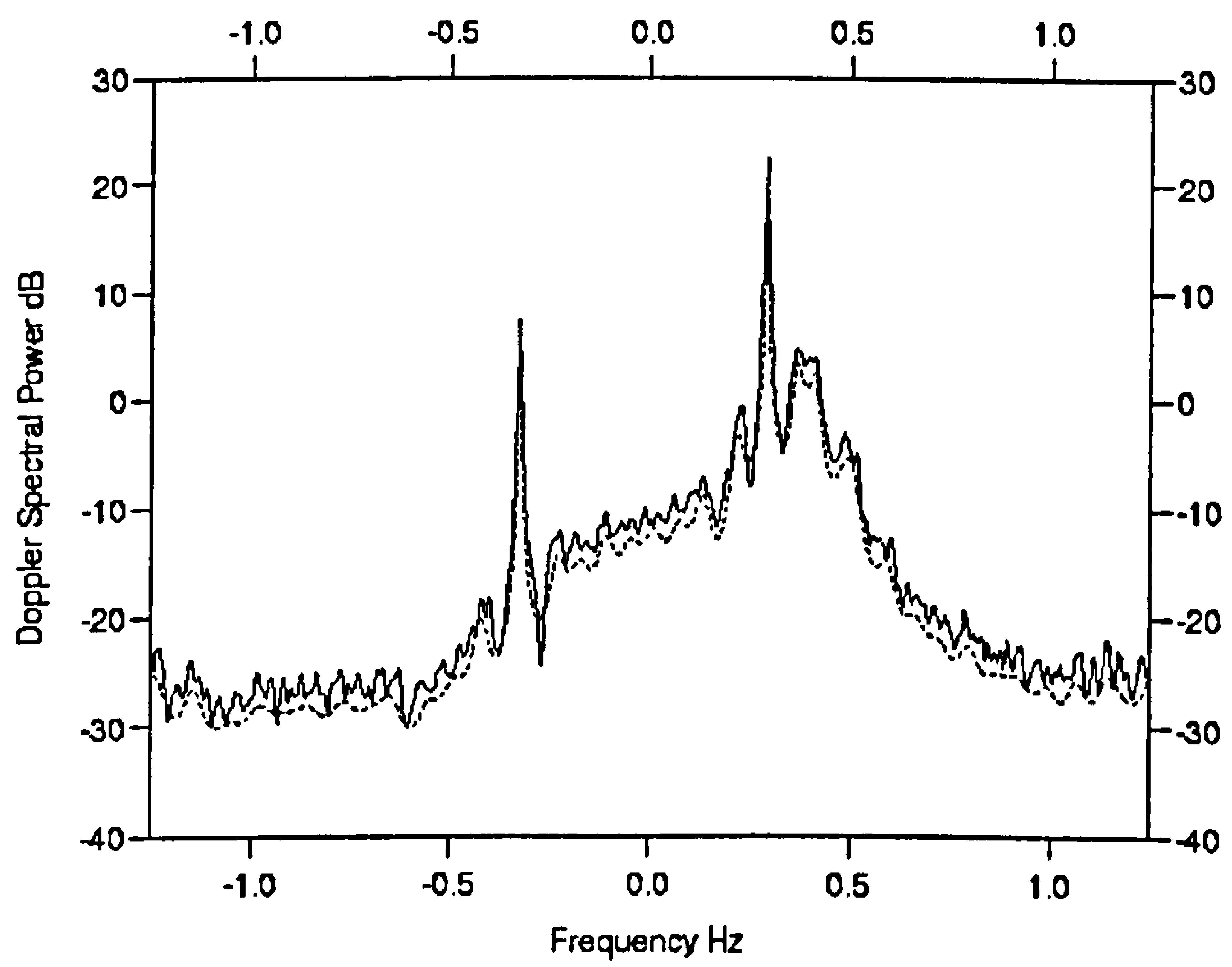

Figure 4.8: Superposition of the periodogram (solid line) and the AR PSD estimate (dotted line) with $N=4608$ and $p=40$, obtained from the PISCES file EB0328.008. 


\subsection{Split peaks}

In the first chapter, we presented the problem of split peaks. When long data time collections are used, split peaks can appear in the Doppler spectrum (see figure 1.2). (This is not the same phenomenon seen in Burg's method (see Chapter 3 section 3.1).) This chapter explains this phenomenon and the modified covariance method is presented as a solution to this problem by reducing the amount of data.

\subsubsection{Problem}

With the OSCR experiments, Wyatt (1994) and later Ledgard and Wyatt (1997c) described the problem of split Bragg peaks and suggested this was a result of current variability. They suggested that this phenomenon could be the result of averaging several spectra over one hour measurement and lead to a smearing effect of the Doppler spectra resulting in a broadening of the main Bragg peak and in some cases resulting in a split peak. Figures 1.2, 4.9 and 4.10 illustrate this problem which distorts the integral under the main Bragg peaks and then the wave parameters and could prevent the inversion process being done if the difference between the two peaks is less than $3 \mathrm{~dB}$. Recall that the first-order part of the Doppler spectrum consists theoretically of two impulse functions symmetrically spaced about the carried frequency (see Chapter 2 section 2.4). This is no longer the case when the peaks are split. The first-order part is distorted.

\subsubsection{Solution}

Figures 1.2, 4.9 and 4.10 show Doppler spectra with split Bragg peaks. Figures 4.11, 4.12 and 4.13 show respectively the corresponding AR spectral estimate resulting from the same measurement but with only one 5-minute measurement. As it can be seen, there are no split peaks in these spectral estimates. 


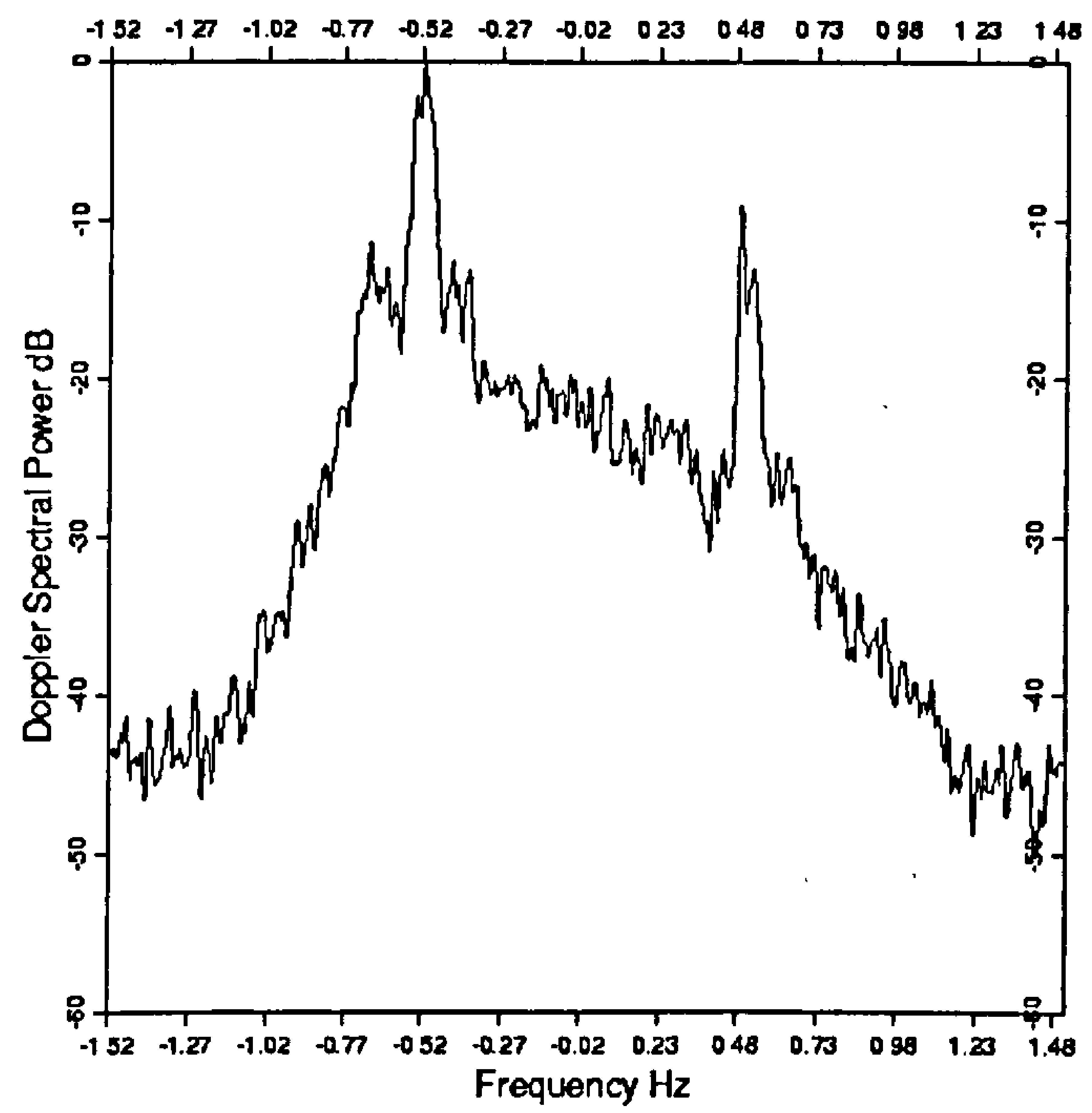

Figure 4.9: Doppler spectrum resulted from the periodogram with full data set, showing split peaks, obtained from the cell 33 (measurement taken at 18pm on 6 January 1996).

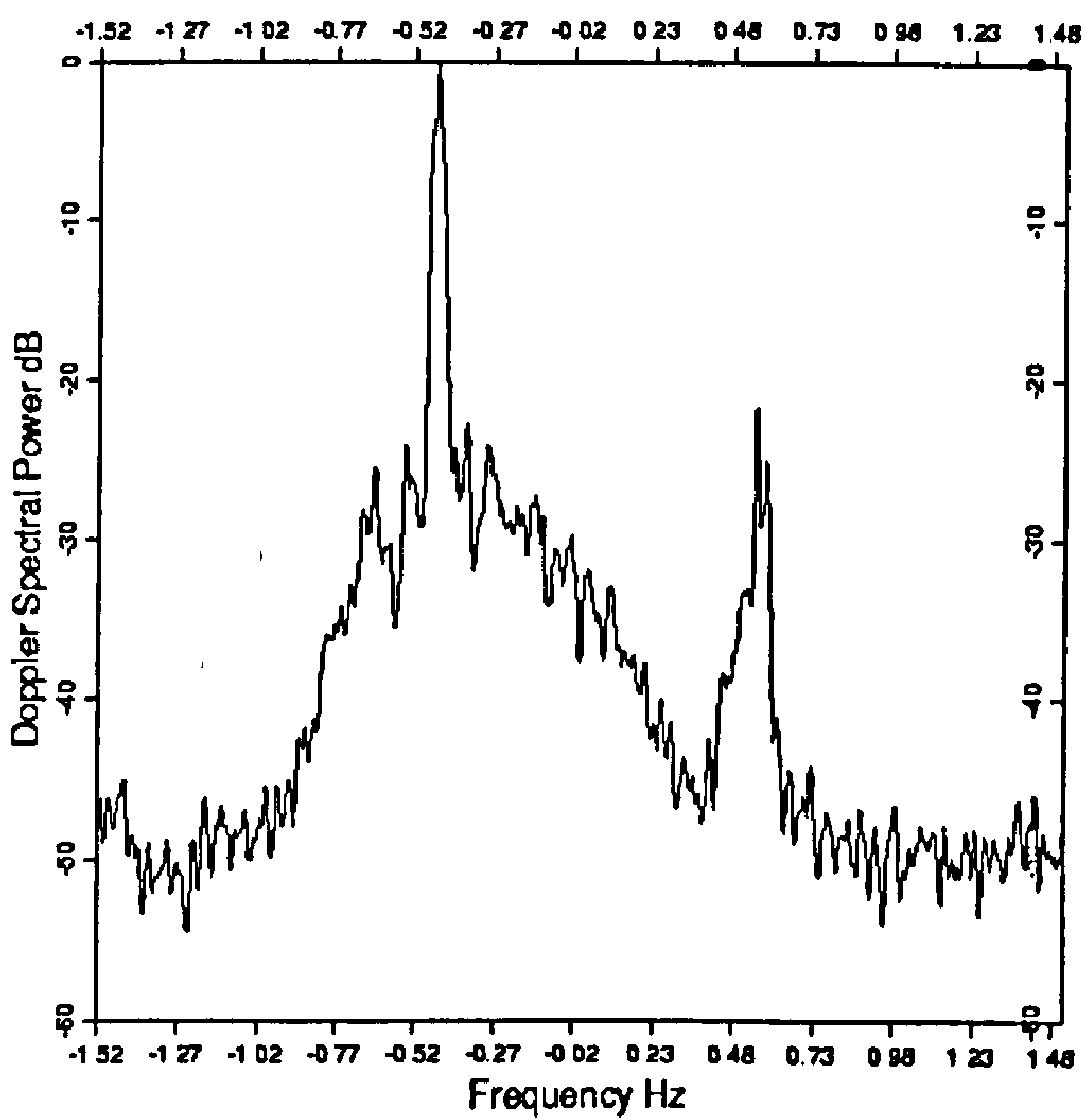

Figure 4.10: Doppler spectrum resulted from the periodogram with full data set, obtained from the cell 54 (measurement taken at 12am on 3 January 1996), showing split peaks. 
Figure 4.14 just shows the regions surrounding the two first-order peaks of the periodogram with full data set shown in figure 1.2 and the three successive AR spectral estimates obtained by the modified covariance method with $N=896$ and $p=40$. As can be seen, the two Bragg peaks move to the left during the hour-long measurement and they remarkably delimit the periodogram Bragg peaks. This was already noticed with the wavelet analysis in Chapter 2 section 2.6.2. The current has varied during this hour and this shows the phenomenon of split Bragg peaks is due to a current variability.

This could have been done with the periodogram. But since in Chapter 2 section 2.3.2, it was pointed out that the 5-minute periodogram was not enough good to provide reliable wave measurements because of its high PSD estimate variance, we would rather use the modified covariance method.

In conclusion, we can say that this problem is due to current variability. One hour measurement can be too long to get both current and wave measurements. This can be reduced by decreasing the time of the measurement and using the modified covariance method.

\subsection{Summary}

From a visual interpretation, this chapter showed good similarities between the periodogram and the AR PSD estimate resulting from the modified covariance method. Now we need to give a quantitative assessment of performance for both spectral techniques in order to validate the modified covariance method. The next chapter will deal with several methods of comparisons. Chapters 6 and 7 will show the results obtained respectively for the OSCR and PISCES systems.

Also, we explained the problem of split peaks which is caused by current variability. This led us to reduce the amount of data in order to solve this problem and emphasized the use of the modified covariance method since it is not preferable to use the periodogram with short data sets. 
The modified covariance method, providing higher frequency resolution (see Chapter 3 section 3.1) gives rise to thinner Bragg peaks and so enables us to avoid the problem of broad Bragg peaks, which occurs with the periodogram.

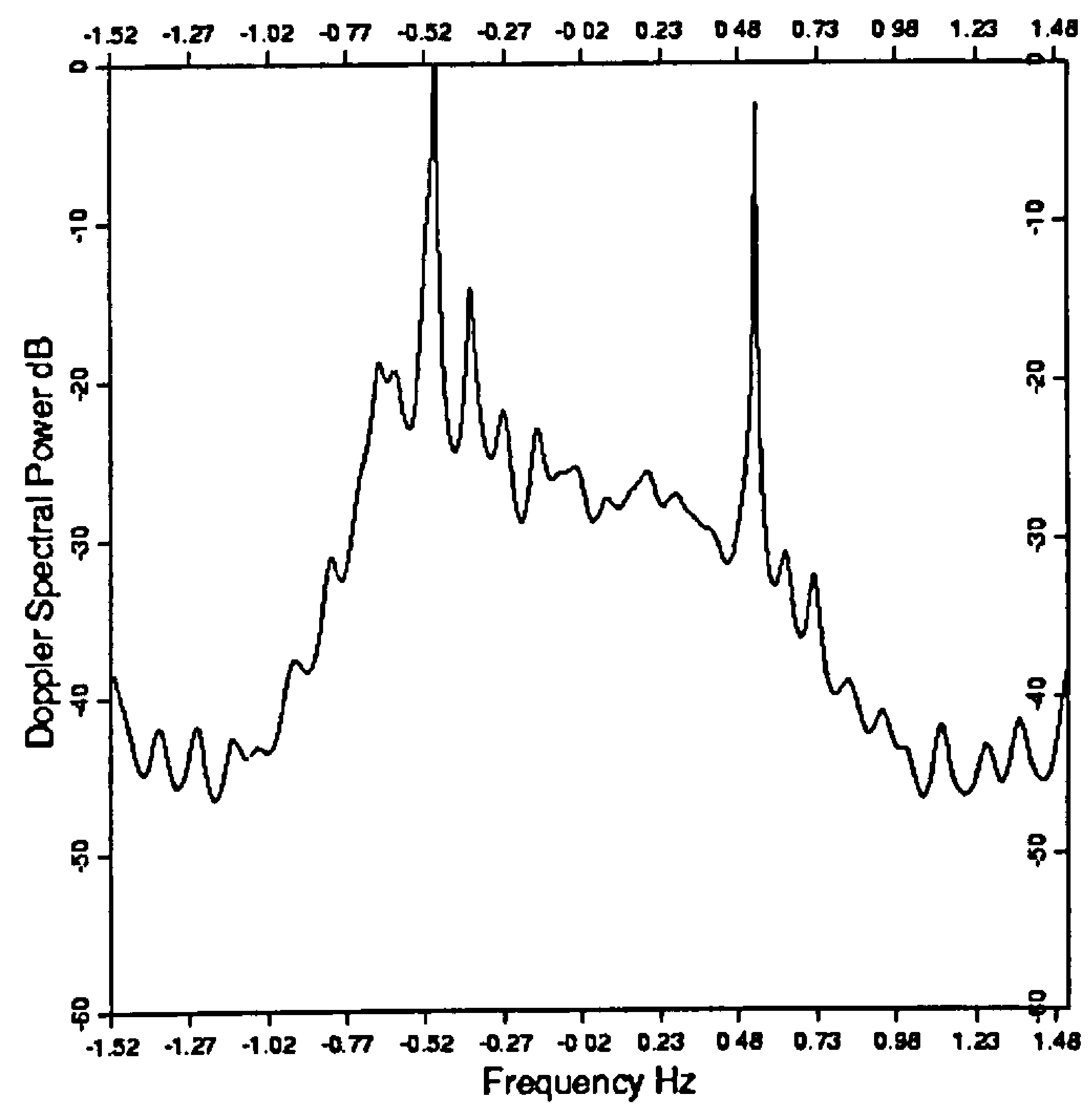

Figure 4.11: Doppler spectrum resulting from the modified covariance method with $N=896$ and $p=40$, obtained from the cell 55 (measurement taken at $18 \mathrm{pm}$ on 6 January 1996), showing no split peaks (see figure 1.2). 


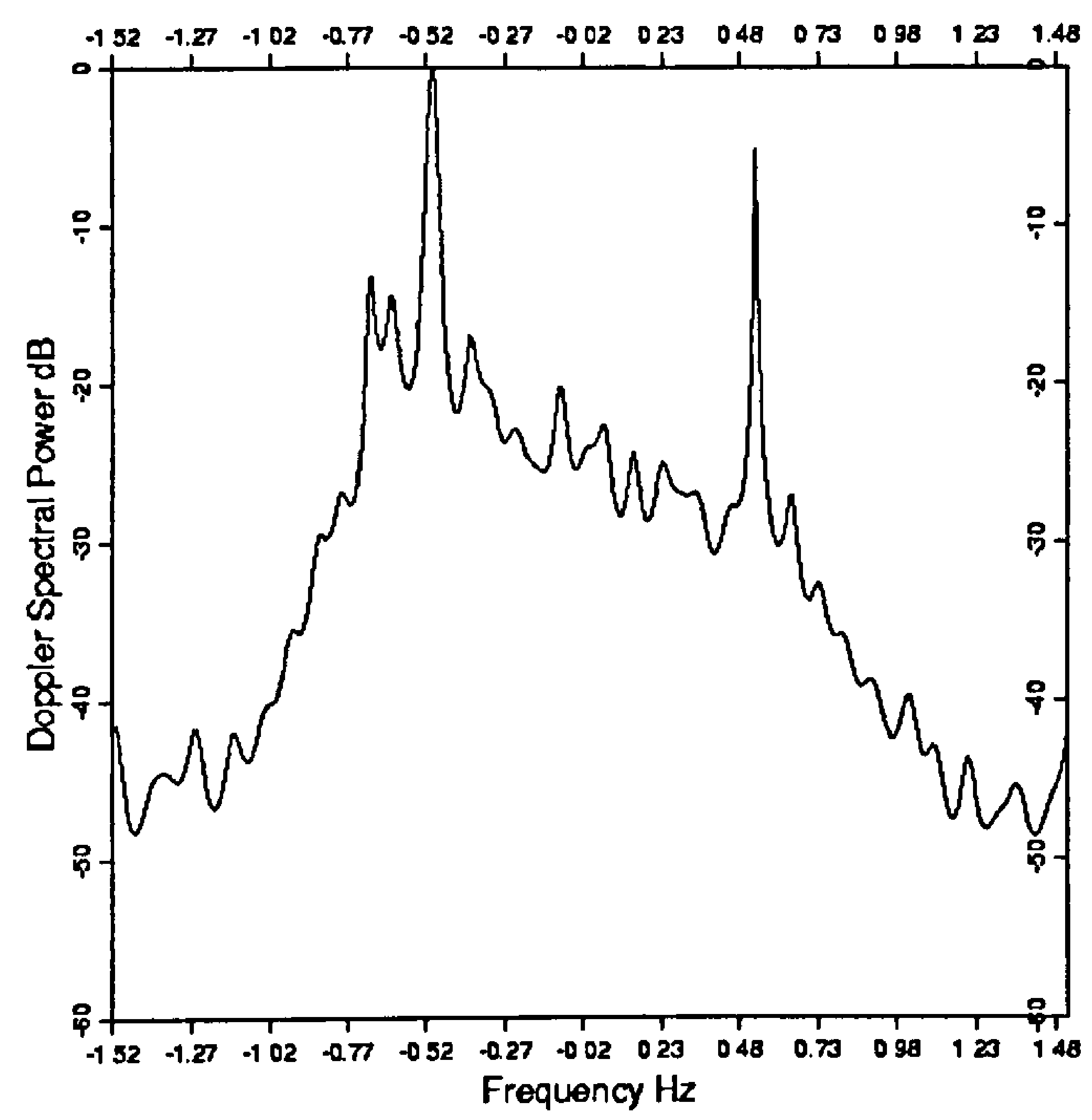

Figure 4.12: Doppler spectrum resulting from the modified covariance method with $N=896$ and $p=40$, obtained from the cell 33 (measurement taken at $18 \mathrm{pm}$ on 6 January 1996), showing no split peaks (see figure 4.9).

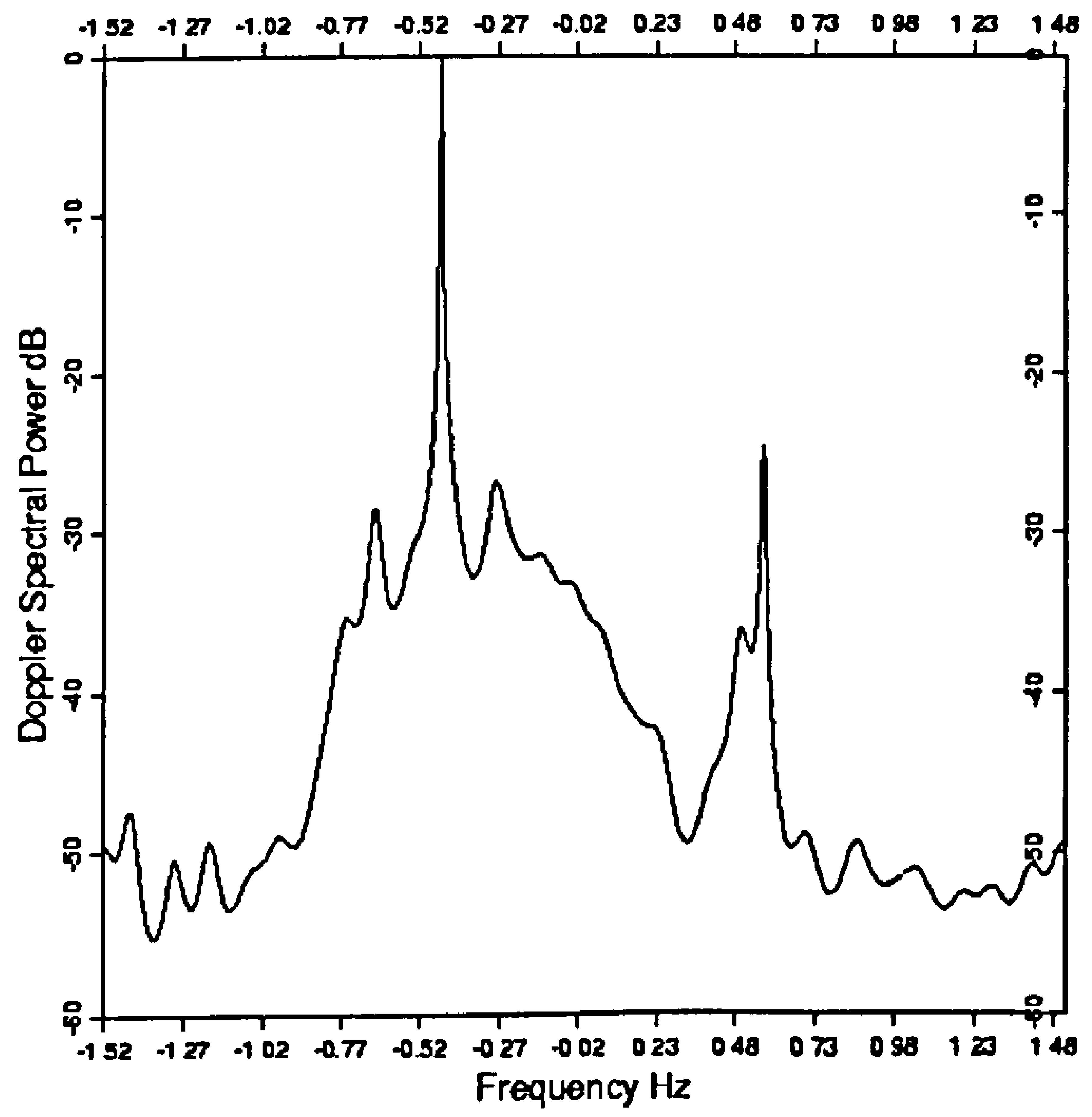

Figure 4.13: Doppler spectrum resulting from the modified covariance method with one 5-minute collection, obtained from the cell 54 (measurement taken at 12am on 3 January 1996), showing no split peaks (see figure 4.10). 

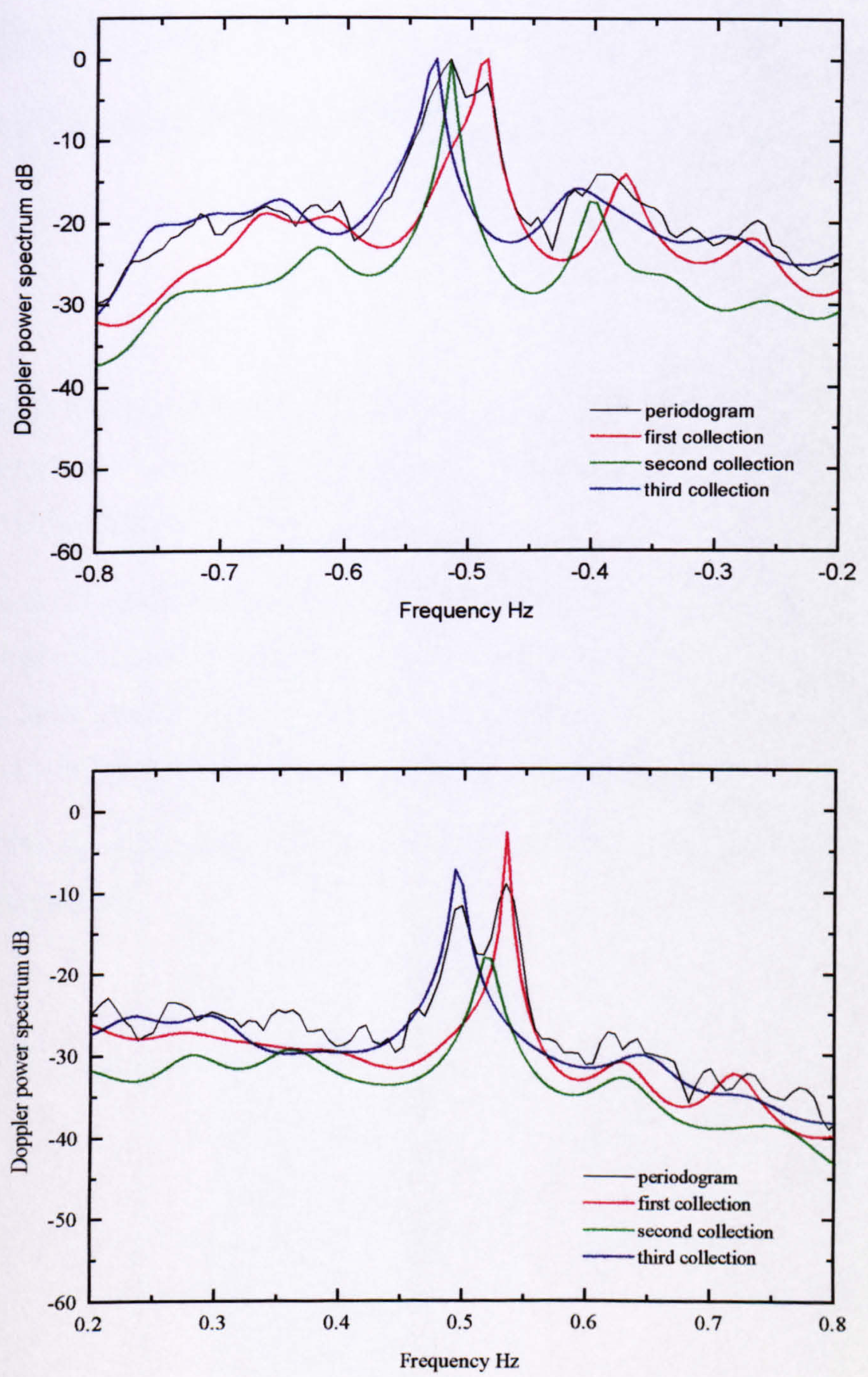

Figure 4.14: Superposition of the periodogram (with full data set) represented by the black line and the three successive AR spectral estimates obtained by the modified covariance method (with $N=896$ and $p=40$ ) represented in red for the first 5minute collection, green for the second and blue for the last. Only the regions surrounding the first-order peaks are shown. 


\section{Chapter 5}

\section{Methodology to Compare the Modified Covariance Method with the Periodogram}

In order to validate the modified covariance method described in the previous chapter, we compare it with the periodogram, the classical spectral technique which is presented in Chapter 2 section 2.3.1.

To do so, the wave parameters obtained from both the periodogram and the modified covariance method are compared, first with a Monte Carlo simulation study and second with wave-buoy measurements. In addition, with the Monte Carlo simulation study, another comparison is done, directly on the spectral estimates.

The different results are presented in Chapters 6 (for the OSCR system) and 7 (for the PISCES system). 


\subsection{Monte Carlo comparison}

The aim of the Monte Carlo simulation study (Vaitkus et al., 1988) is to assess the performance of the different spectral methods. The different steps of this study are illustrated in figure 5.1. From the simulation model, a time domain signal is produced and transformed into a spectral model which is compared to the measured spectrum considered as the "true" spectrum. The overall error that results can then be used to assess the quality of the spectral model.

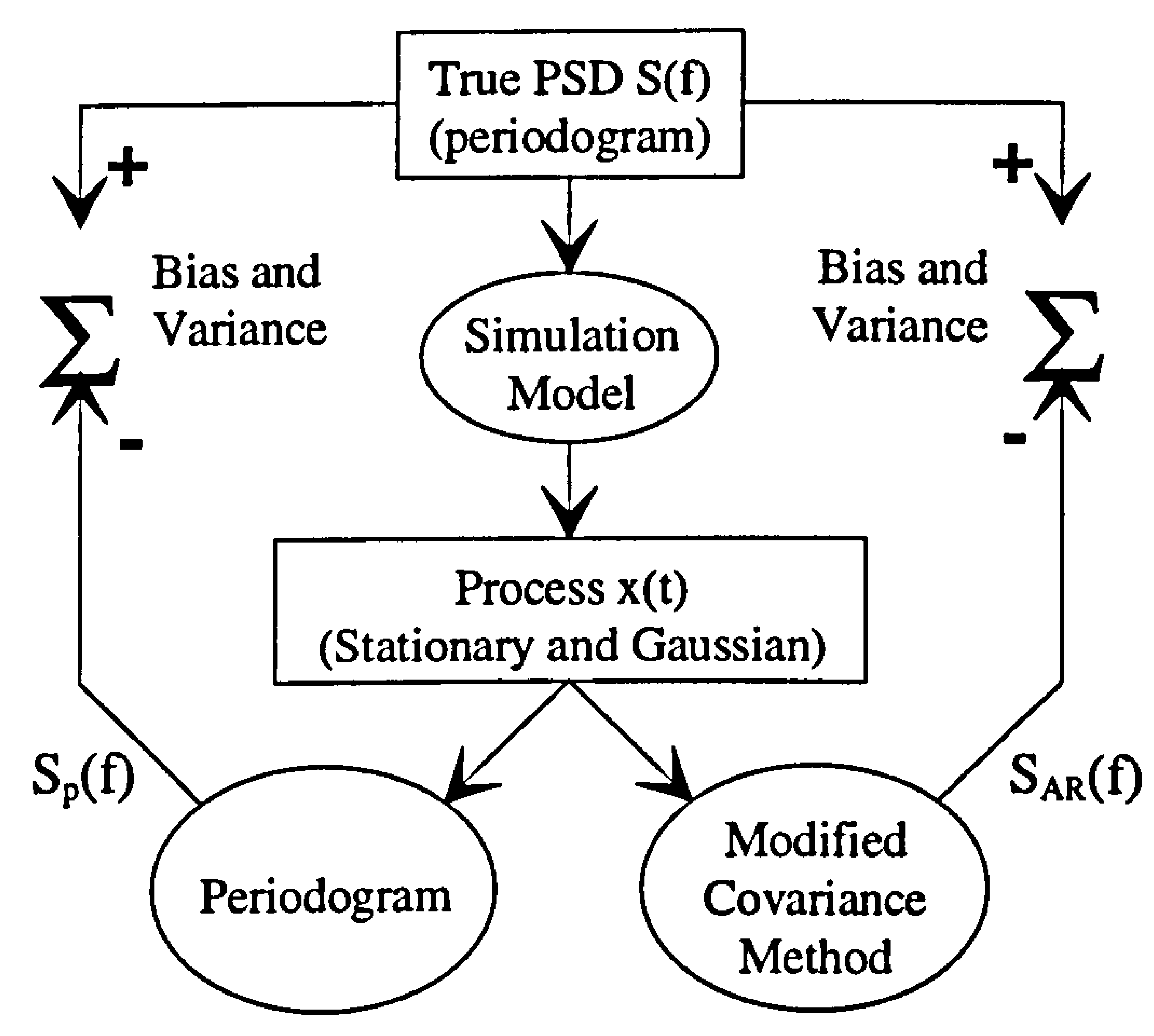

Figure 5.1: Flow chart illustrating the different steps of the Monte Carlo study.

\subsubsection{Simulated data}

We assume a given power spectrum density (PSD) function $S(f)$, which is considered as the "true" $S(f)$. 
The simulated signal is obtained by $x(k)=\frac{1}{n} \sum_{j=-n / 2}^{n / 2} R_{j} e^{i k \frac{2 \pi j}{n}}$, for $k=1 \ldots n$ ( $n$ being the dimension of the signal) where the $R_{j}$ 's are complex orthogonal random variables with zero-mean and with a variance of $\operatorname{Var}\left(R_{i}\right)=S\left(\frac{2 \pi j}{n}\right)$; in another words, we have $R_{j}=A_{j}+i B_{j}$ where $A_{j}$ and $B_{j}$ are proportional to a normal distribution such as:

$$
\left\{\begin{array}{l}
A_{j} \approx \sqrt{S\left(\frac{2 \pi j}{n}\right)} \times N(0,1) \\
B_{j} \approx \sqrt{S\left(\frac{2 \pi j}{n}\right)} \times N(0,1)
\end{array}\right.
$$

The resulting process $x(k)$ is a zero-mean, second-order stationary, complex Gaussian signal, having the given PSD.

The "true" $S(f)$ is the periodogram since it contains both the first- and second-order effects. It is compatible with the simulation model because if $x(k)$ was real, the two normal variables could be replaced by one random variable following a $\chi^{2}$ (chisquare) distribution. The sampling distribution of the periodogram (with one segment) is a $\chi^{2}$ distribution, assuming that the process from the start is Gaussian (Priestley, 1981).

Given that the "true" $S(f)$ is composed of 512 points, the simulation model can only generate independent data time-series of 512 samples.

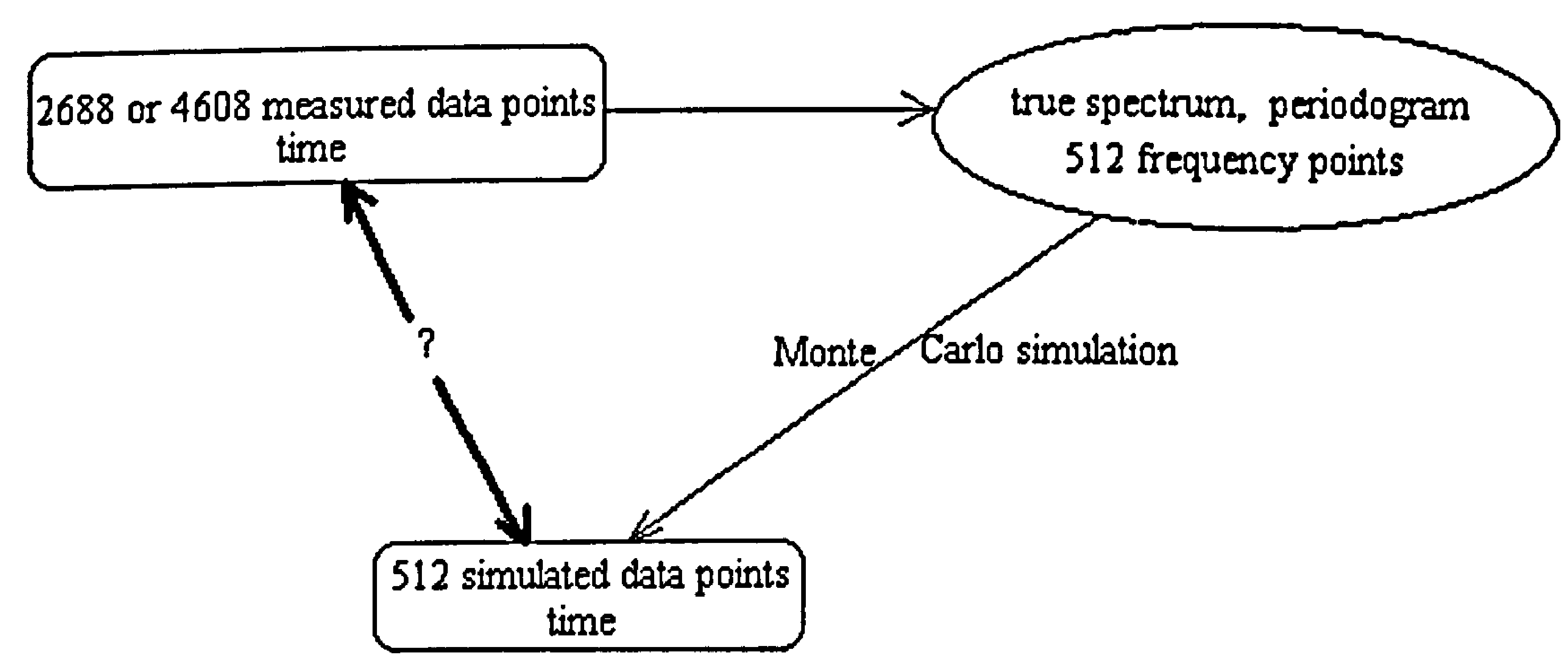

Figure 5.2: Flow chart showing the main steps of the Monte Carlo simulation to get the simulated data. 
As can be seen in figure 5.2, we cannot apply the periodogram to the simulated data in the same way as it was done on the 2688 or 4608 measured data points respectively for the OSCR and PISCES systems. Overlapping is not possible. Because overlapped data sets have some correlation, we do not need as many averages using our independent sets as is required with both OSCR and PISCES systems. We use equations (2.8) and (2.9) (seen in Chapter 2, section 2.3.2), which describe the consequence of overlapping segments. By equalizing these two equations, we estimate that 9 and 23 sets are sufficient for the OSCR and PISCES systems respectively.

Since the OSCR system is more restrictive in the sense of number of averages and since disk space and computation time are too demanding for the PISCES system, we apply the Monte Carlo simulation to the OSCR system only.

We need to check if the observed data, collected from both systems (PISCES and OSCR), are stationary and Gaussian in order to apply this simulation model. In Chapter 2, these properties were treated in section 2.6. We pointed out the difficulty of showing the stationarity. Here we can make a qualitative judgement. As the simulation model guarantees the stationarity of the resulting signal, we can compare the variations of the actual signal with the simulated signal. Figure 5.3 shows one example of simulated data obtained from the periodogram seen in figure 2.2 (Chapter 2, section 2.3.1) with 512 points and figure 5.4 shows the three signals with 896 points which produced this periodogram. As can be seen, the real and imaginary parts for each case vary in a similar fashion; the actual signal varies as much as the simulated signal. So we can assume that the observed data are stationary and then apply this simulation study to our case. However, we can notice in the actual signal that the maximum amplitude is different for each 896-sample set and for the last collection, the amplitude is increasing during time. In the future, it will be interesting to investigate further these variations and hence the stationarity of the HF radar data with the Wavelet analysis presented in Chapter 2 section 2.6.2. 


\subsubsection{Error Measurements}

Since the true PSD function, $S(f)$, is known, it is straightforward to get the bias and variance, which are used here to describe the quality of the spectral estimates. We can obtain these error parameters in two different ways: directly on the spectral estimates, by comparing them with the "true" spectrum $S(f)$, and on the ocean wave parameters, by extracting them from the different spectral estimates and the "true" $s(f)$.

\subsubsection{Spectral estimates}

By definition, the bias is the deviation of the ensemble averaged spectral estimate from the true value:

$$
\operatorname{Bias}(\hat{S}(f))=\frac{E[\hat{S}(f)]-S(f)}{S(f)}
$$

where $S(f)$ is the spectral density, $\hat{S}(f)$ the estimate and $E$ the expectation operator. The variance measures the spread in the error and is given by

$$
\operatorname{Var}(\hat{S}(f))=E\left[\frac{\hat{S}(f)-E(\hat{S}(f))}{S(f)}\right]^{2} .
$$

The mean square error incorporates both the bias and the variance:

$$
\operatorname{MSE}(\hat{S}(f))=E\left[\frac{\hat{S}(f)-S(f)}{S(f)}\right]^{2}=\operatorname{Var}(\hat{S}(f))+\operatorname{Bias}^{2}(\hat{S}(f))
$$
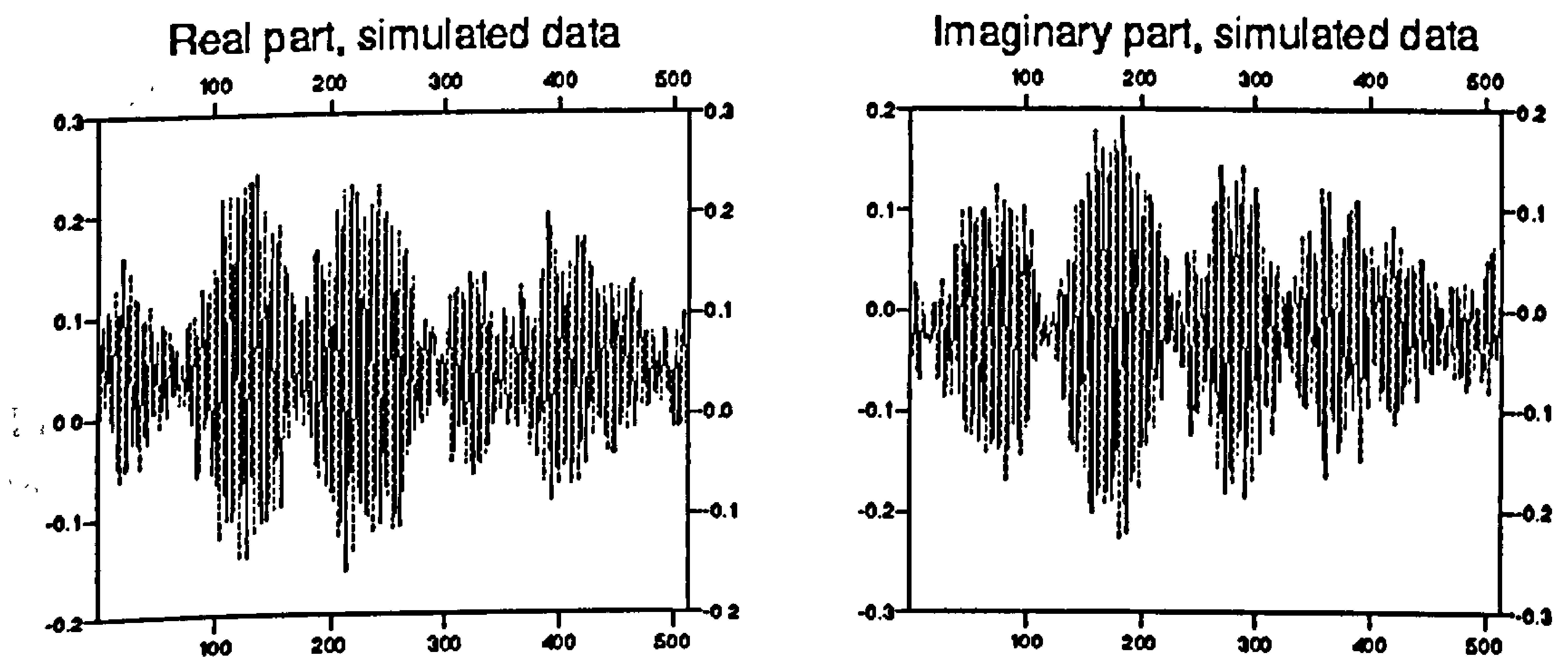

Figure 5.3: Simulated signal obtained from the periodogram shown in figure 2.2. 
Real part, OSCR data (first collection)

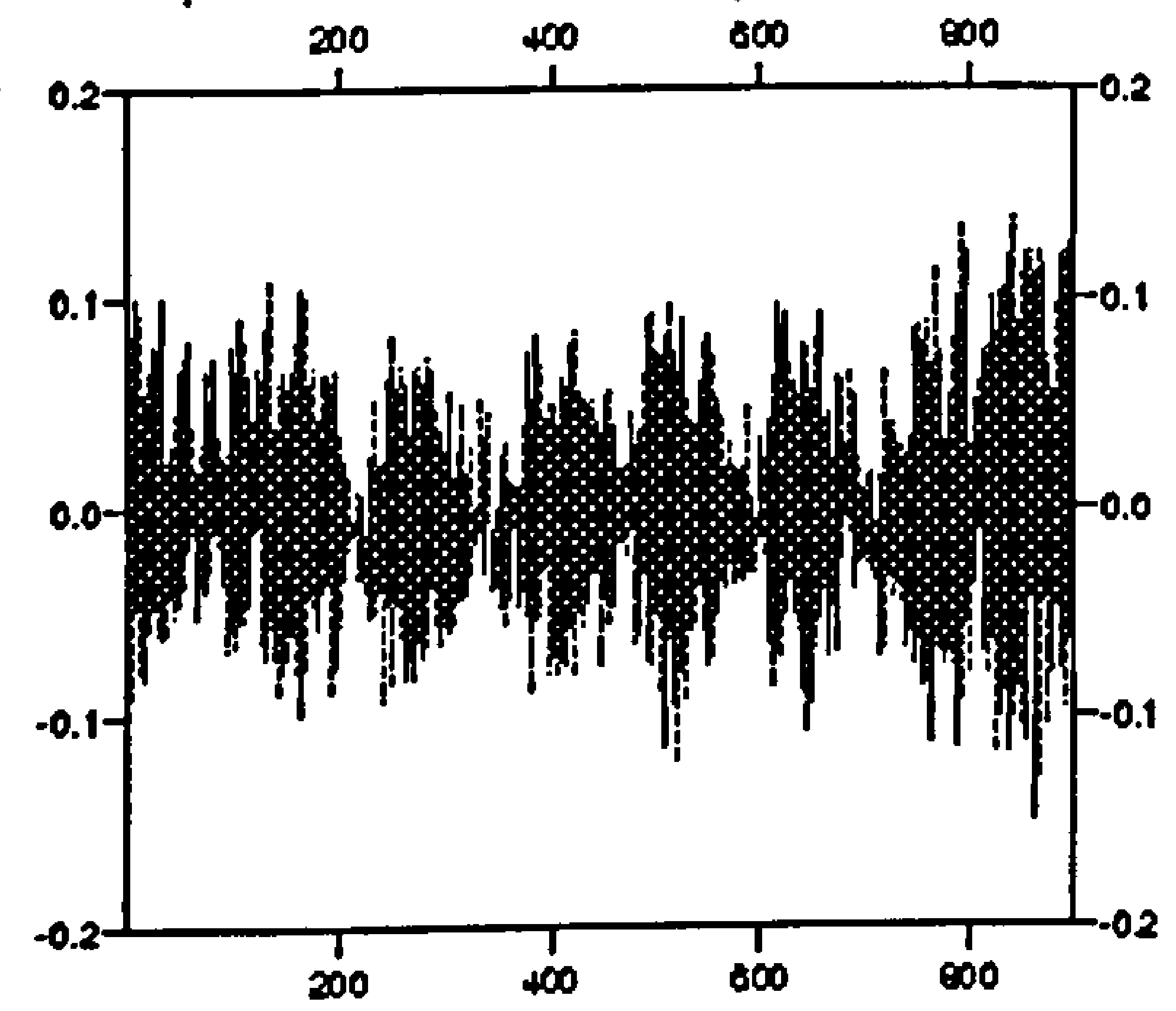

Real part, OSCR data (second collection)

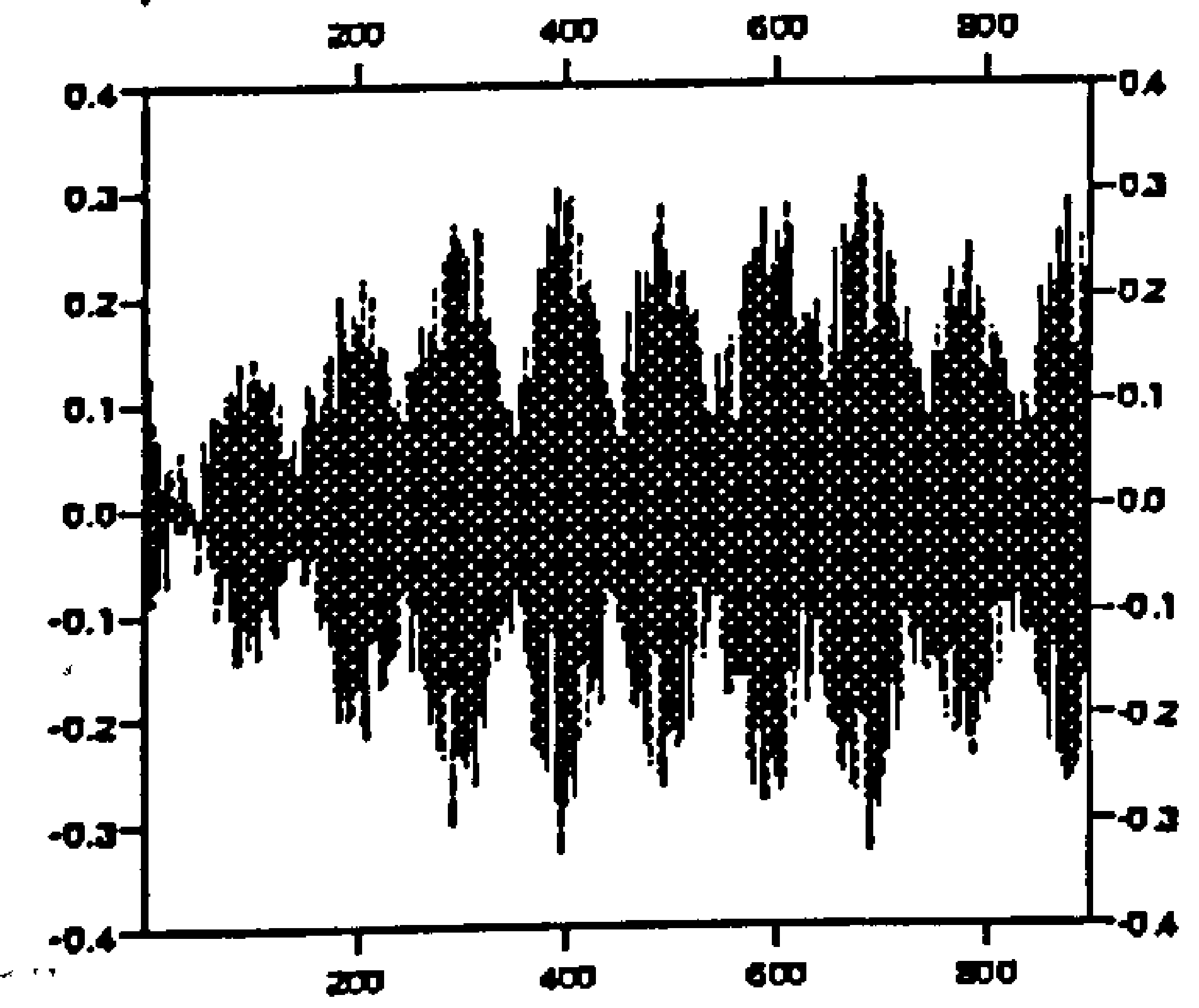

Real part, OSCR data (third collection)

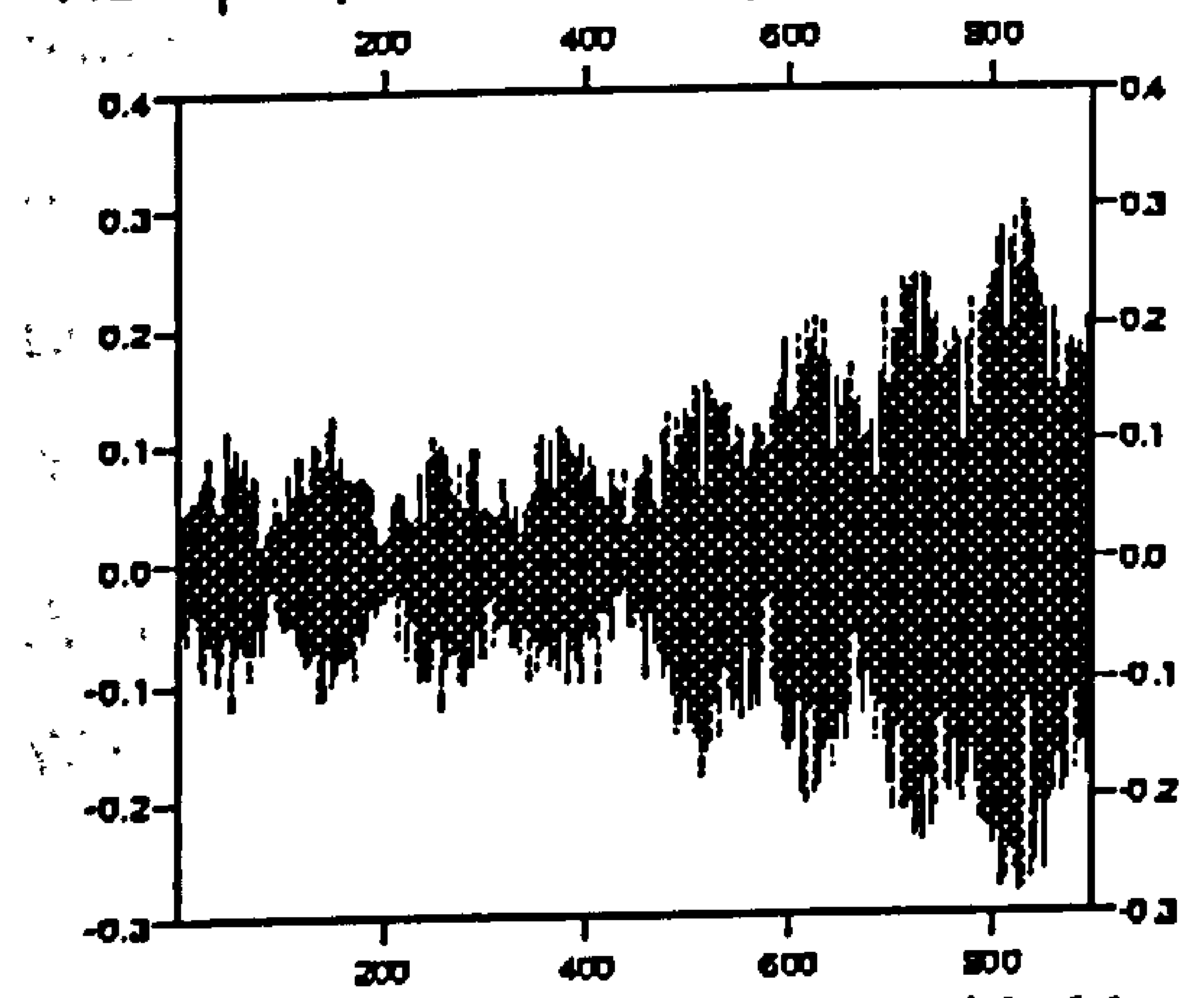

Imaginary part, OSCR data (first collection)

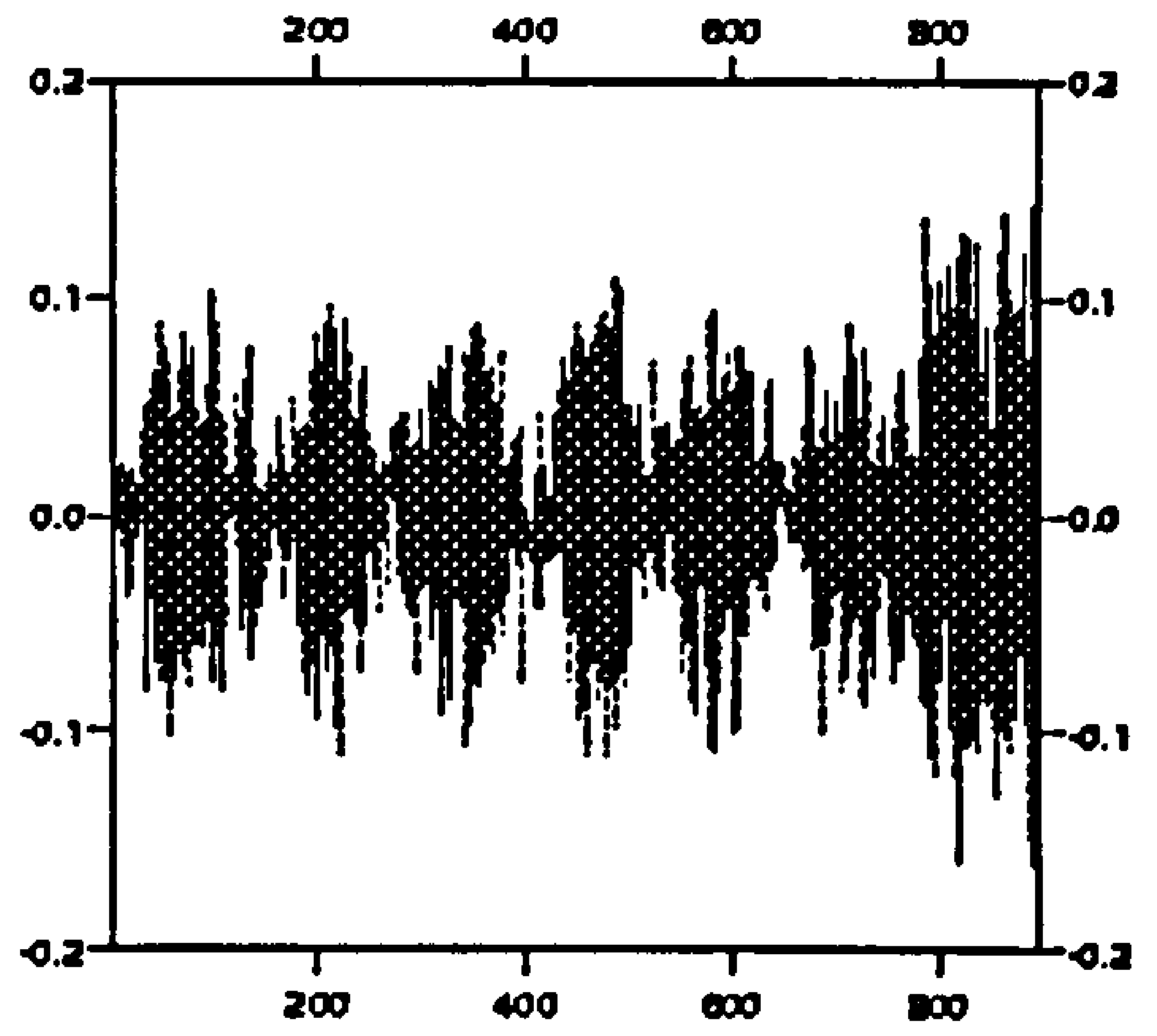

Imaginary part, OSCR data (second collection)

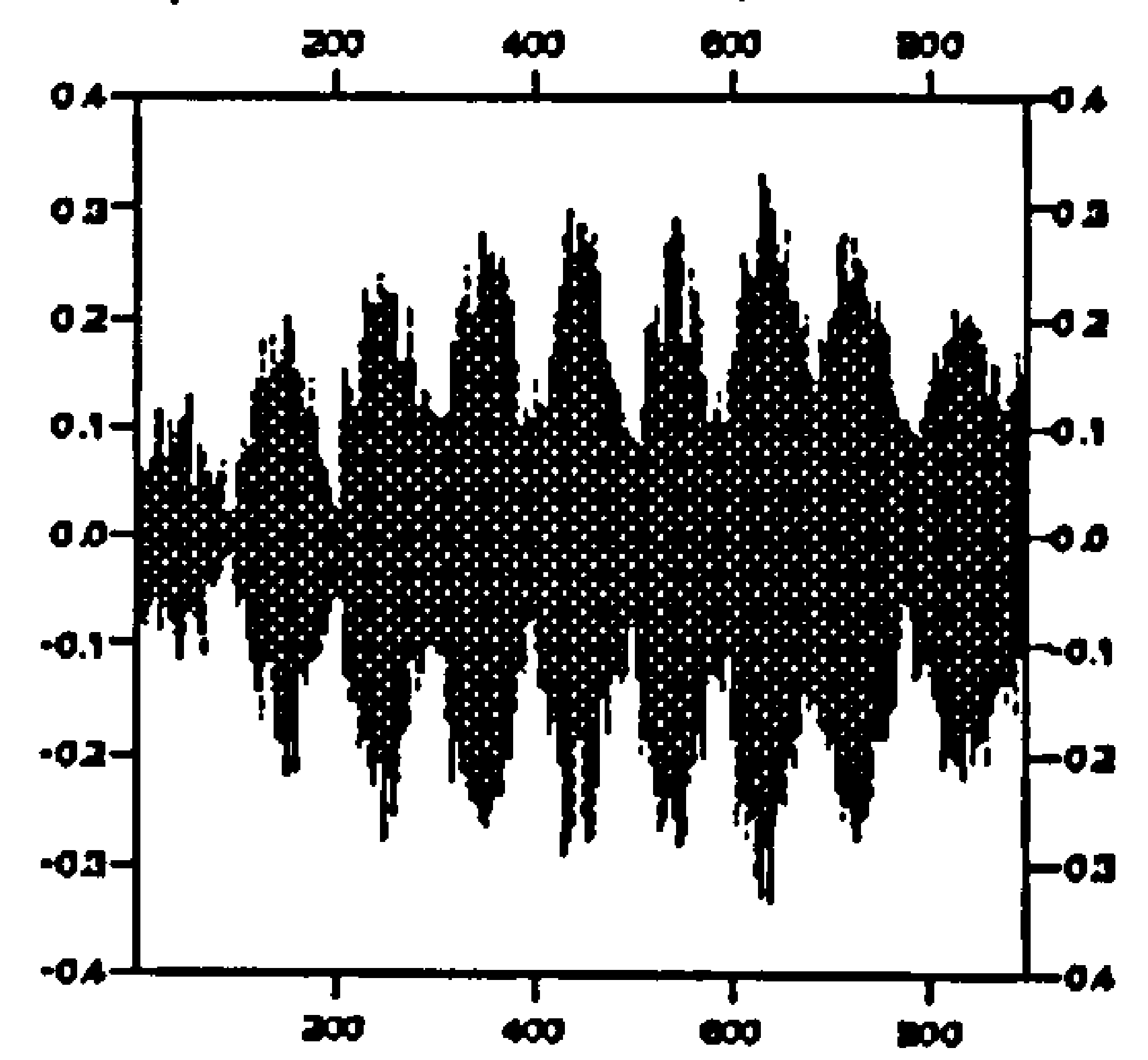

Imaginary part, OSCR data (third collection)

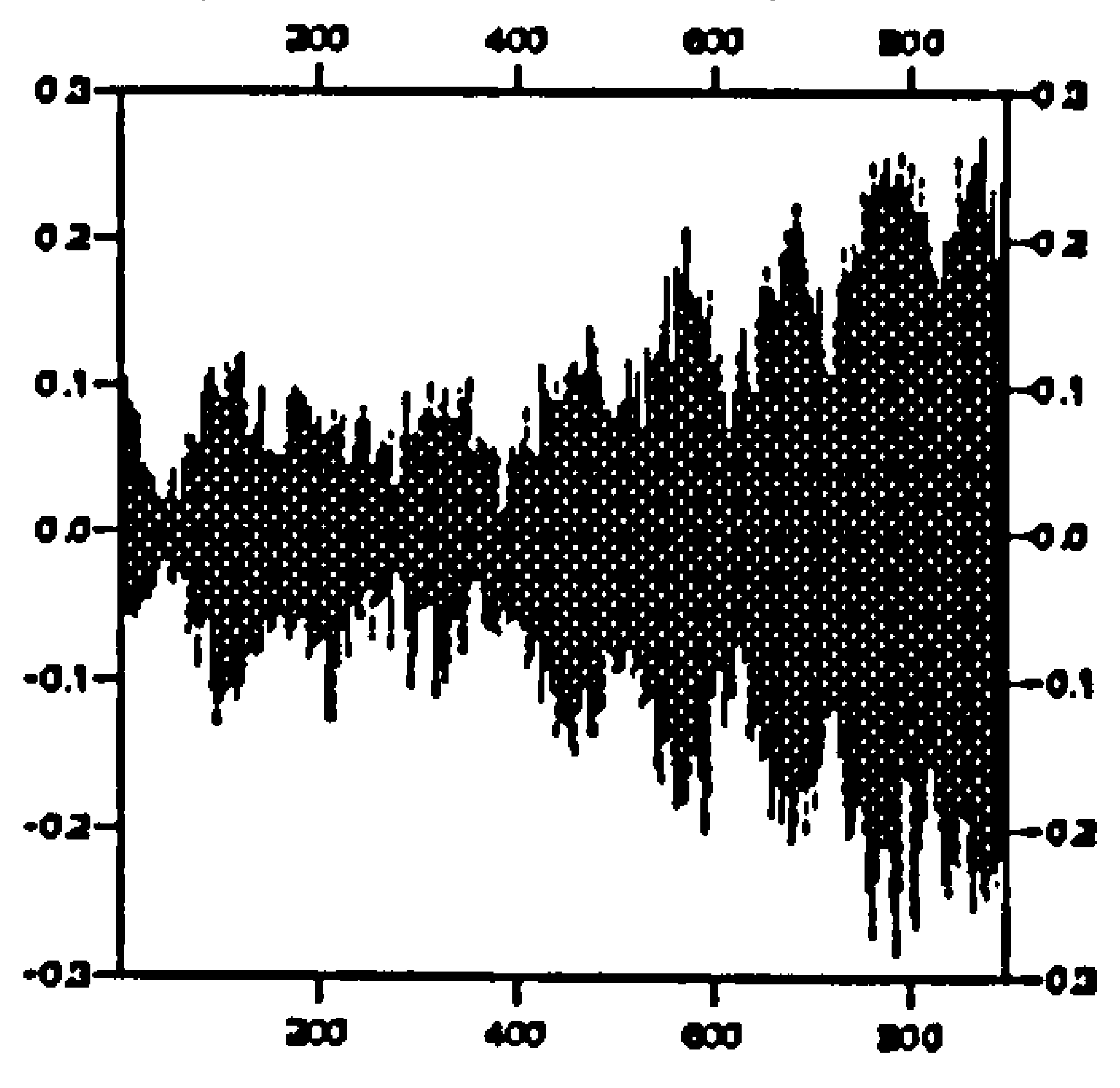

Figure 5.4: Observed data provided by the OSCR system (measurement taken at $3 \mathrm{pm}$ on 27 December 1995), the three 5-minute measurements are shown. 
These parameters are functions of frequency. By integrating respectively the bias, the variance and the mean square error over the frequency domain, the integrated bias (IB), the integrated variance (IVAR) and the integrated mean square error (IMSE) can be obtained. These integrated parameters represent the global deviation. An alternative set of integrated parameters can be obtained by first taking the logarithm of the different PSD functions, which is related to the $\mathrm{dB}$ scale. These will be referred to

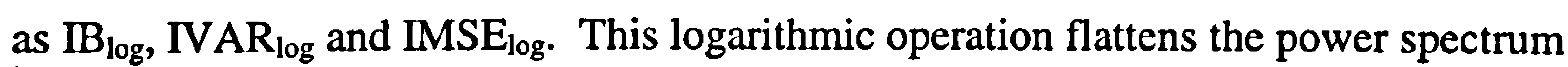
and the differences surrounding the first-order peaks are emphasized.

Given that the second-order effect is of greatest interest, all these integrated parameters are calculated with the integration range which is a neighborhood of the main peak but does not include the peak itself, in other words the maximum range used for wave parameter estimation given in chapter 2 sections 2.4 .1 for the significant wave height $H_{S}$ and 2.4.2 for the mean period $T_{\omega}$.

\subsubsection{Wave parameters}

We can also use the simulation to determine the bias and the variance of wave parameters estimated from the synthesized signal power spectra. The wave parameters we consider are the wind direction $\theta_{\omega}$ (Chapter 2, section 2.3), the mean wave period $T_{\omega}$ (Chapter 2, section 2.4.2) and the significant wave height $H_{S}$ (Chapter 2, section 2.4.1). $\theta_{\omega}$ is calculated from the difference in amplitude of the two first-order peaks (Wyatt et al., 1996). The other two wave parameters $\left(H_{S}\right.$ and $\left.T_{\omega}\right)$ are extracted from the second-order contribution when normalized with respect to the first-order spectrum (Wyatt, 1986).

We get similar formulas for the bias and the variance:

$$
\begin{gathered}
\operatorname{Bias}(\hat{\xi})=\frac{E[\hat{\xi}]-\xi}{\xi}, \\
\operatorname{Var}(\hat{\xi})=E\left[\frac{\hat{\xi}-E(\hat{\xi})}{\xi}\right]^{2},
\end{gathered}
$$

where $\xi \in\left\{\theta_{\omega}, T, H_{S}\right\}$ is the true wave parameter and $\hat{\xi}$ is the estimate. 


\subsubsection{Sample size problem}

At this stage, a question arises : how many spectral estimates must we take, or how many processes $x(t)$ must we generate, in order to obtain acceptable values for the different error parameters?

This is a well-known problem: the size of sample (Cochran et al., 1989).

In our case, we want to find the number of spectral estimates, $n$, necessary to obtain, for a $95 \%$ confidence interval, an estimate of the mean square error, $\overline{M S E}$, within $\pm \delta$ $(\delta \leq 0.005)$ of the true value for the MSE.

We consider here the mean square error seen in equation (5.4) calculated at each frequency because it contains both bias and variance parameters. Given the great importance of the second-order part of the spectrum, it is sufficient to solve this problem at 4 different frequencies: the 2 frequency points determining the first-order peak and the other 2 delimiting the integration range.

Then, for each frequency, we have

$$
\overline{M S E}=\frac{1}{n} \sum_{i=1}^{n}\left(X_{i}-\mu\right)^{2}
$$

where $X_{1} \ldots X_{n}$ represent the spectral estimates and $\mu$ is the true value for $\left(X_{i}\right)_{i=1 . n}$. This definition of the mean square error is slightly different from the definition (5.4): there is no normalization with $\mu$ for simplification purposes.

Since $n$ is large, we can assume that $X_{i}$ follows a normal distribution:

$$
X_{i} \approx N\left(\mu, \sigma^{2}\right) \text {, with } \sigma^{2} \text { known. }
$$

In fact, $\sigma^{2}$ is estimated by

$$
\sigma^{2}=\frac{1}{n-1} \sum_{i=1}^{n}\left(X_{i}-\bar{X}\right)^{2}
$$

where $\bar{X}=\frac{1}{n} \sum_{i=1}^{n} X_{i}$.

Then, we have $\sum_{i=1}^{n}\left(X_{i}-\mu\right)^{2} \approx \sigma^{2} \chi_{n}^{2}$ and so

$$
\overline{M S E} \approx \frac{\sigma^{2}}{n} \chi_{n}^{2}
$$


Thus, $\quad\left\{\begin{array}{c}E(\overline{M S E})=\sigma^{2} \\ \operatorname{Var}(\overline{M S E})=\frac{\sigma^{4}}{n^{2}} \operatorname{Var}\left(\chi_{n}^{2}\right)=\frac{2 \sigma^{4}}{n}\end{array}\right.$.

Hence, for an $95 \%$ confidence interval, we must have

$$
\begin{gathered}
\delta \geq 2 \sqrt{\frac{2 \sigma^{4}}{n}} \\
\text { or } n \geq \frac{8 \sigma^{4}}{\delta^{2}} .
\end{gathered}
$$

This last inequality (5.11) was tested with different values of $n$, for each spectral technique. We found that 4000 spectral estimates are sufficient to obtain acceptable error measurements.

\subsection{Wave-buoy comparisons}

Wave buoys have been used to assess the use of HF radar systems for measuring the ocean wave directional spectrum (Wyatt, 1991).

From the periodogram and the modified covariance method, we extract three wave parameters: the wind direction $\theta_{\omega}$, the mean wave period $T_{\omega}$ and the significant waveheight $H_{S}$, and compare them with the wave-buoy measurements.

\subsubsection{Description and locations}

A wave-buoy system measures directly the directional wave spectrum (Tucker, 1991) by following the motion of the water particles. An internal processor computes the directional wave spectrum, the heave spectrum and the directional parameters. These data are then transmitted over an HF radio link or recorded on board the buoy. From these data, the two dimensional wave spectrum is estimated (see Chapter 2 equation 2.18). The heave gives $S(f)$. The pitch and roll contain the information on direction, that provides $G(f, \theta)$. 
For the PISCES experiment (Wyatt, 1991), a WAVEC directional buoy, from which we will use wave measurements for comparison, and two waveriders have been used (see fig. 5.5). The WAVEC directional buoy measures the slope of the sea surface in two directions (such as North and East) at the same point.

Concerning the OSCR experiment (Wyatt et al., 1997), two directional waveriders were deployed. They are located as shown in figure 5.6 which contains the radar measurement cells as well. For our comparisons, we use the waverider located between cells 36 and 54, marked as D3. It measures the vertical and horizontal displacements of a surface particle.

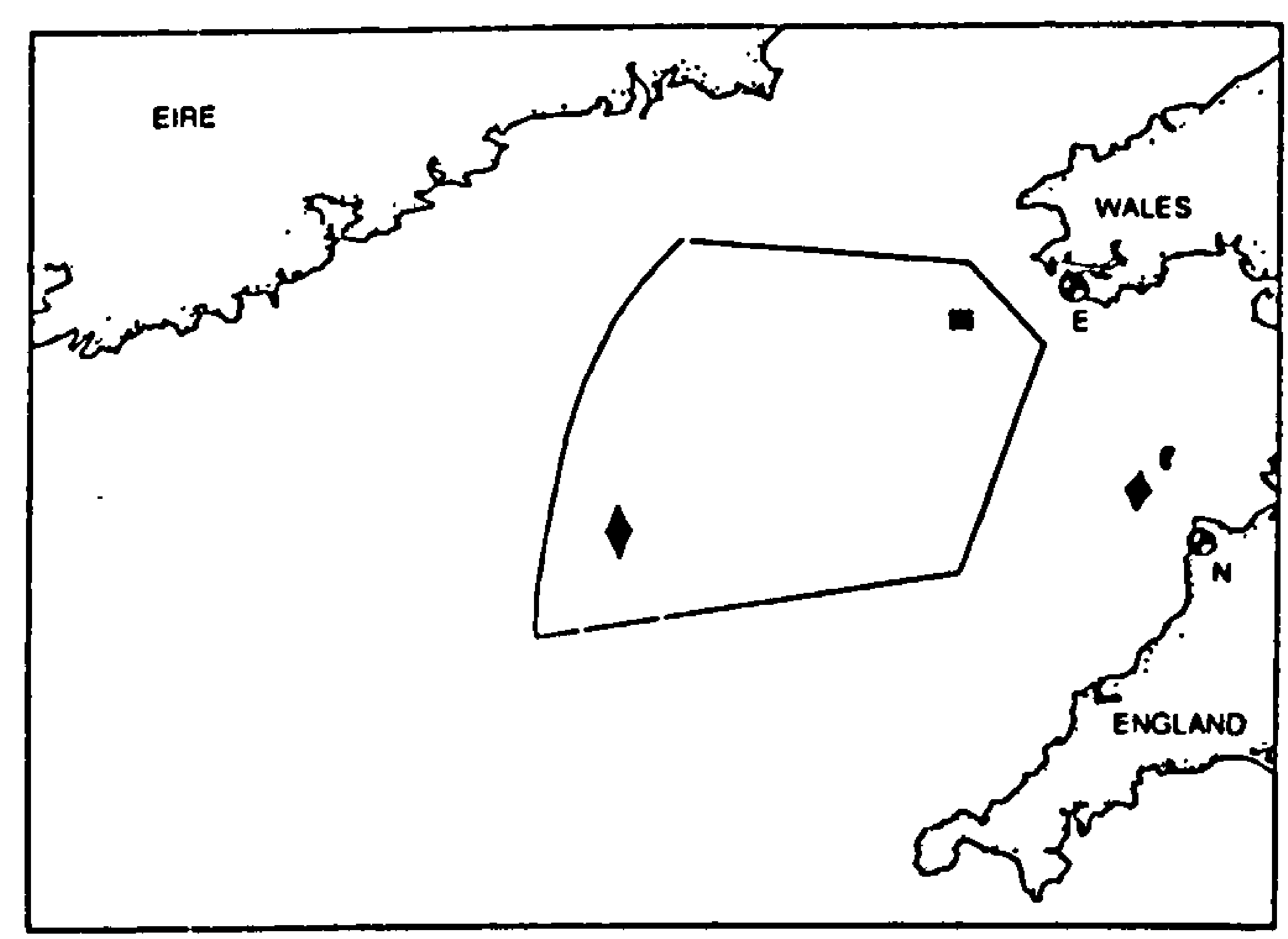

Figure 5.5: PISCES experiment. Map showing radar sites $(\boldsymbol{)})$ at East Blockhouse, southwest Wales (E) and Nabor point, North Devon (N); buoy deployments, WAVEC $(\square)$ and waveriders $(\diamond)$ and the area of dual-radar coverage.

\subsubsection{Error measurements}

Two methods are proposed here. One considers the wave buoy measurements as "sea truth" measurements. The second one is a symmetric regression which takes into account errors coming from both systems (radar and wave buoy system).

We treat separately the wind direction $\theta_{\omega}$ by analyzing the errors in the complex plane (Mardia, 1972) because here the "true" angles are not fixed (as for the Monte 
Carlo simulation study). Instead of working with angles, we calculate the distance error for each measurement:

$$
d\left(z_{w b}, z_{r}\right)=\sqrt{\left(\cos \theta_{w b}-\cos \theta_{r}\right)^{2}+\left(\sin \theta_{w b}-\sin \theta_{r}\right)^{2}} .
$$

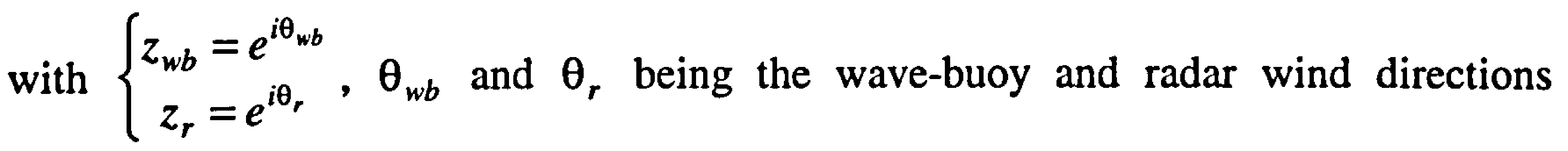
respectively. This is illustrated in figure 5.7.

Then, the mean distance is given by:

$$
\bar{d}=\frac{1}{M} \sum_{i=1}^{M} d_{i}
$$

and the distance variance by:

$$
\operatorname{Var}(d)=\frac{1}{M} \sum_{i=1}^{M}\left(\bar{d}-d_{i}\right)^{2}
$$

where $M$ is the number of measurements.

Note that

$$
d=1 \Leftrightarrow\left|\theta_{w b}-\theta_{r}\right|=60^{\circ}
$$

The mean and the standard deviation can be obtained in degrees by:

$$
\begin{aligned}
& \operatorname{mean}_{\theta}=\bar{d} \times 60 \\
& \text { and } \sigma_{\theta}=\sqrt{\operatorname{Var}(d)} \times 60
\end{aligned}
$$




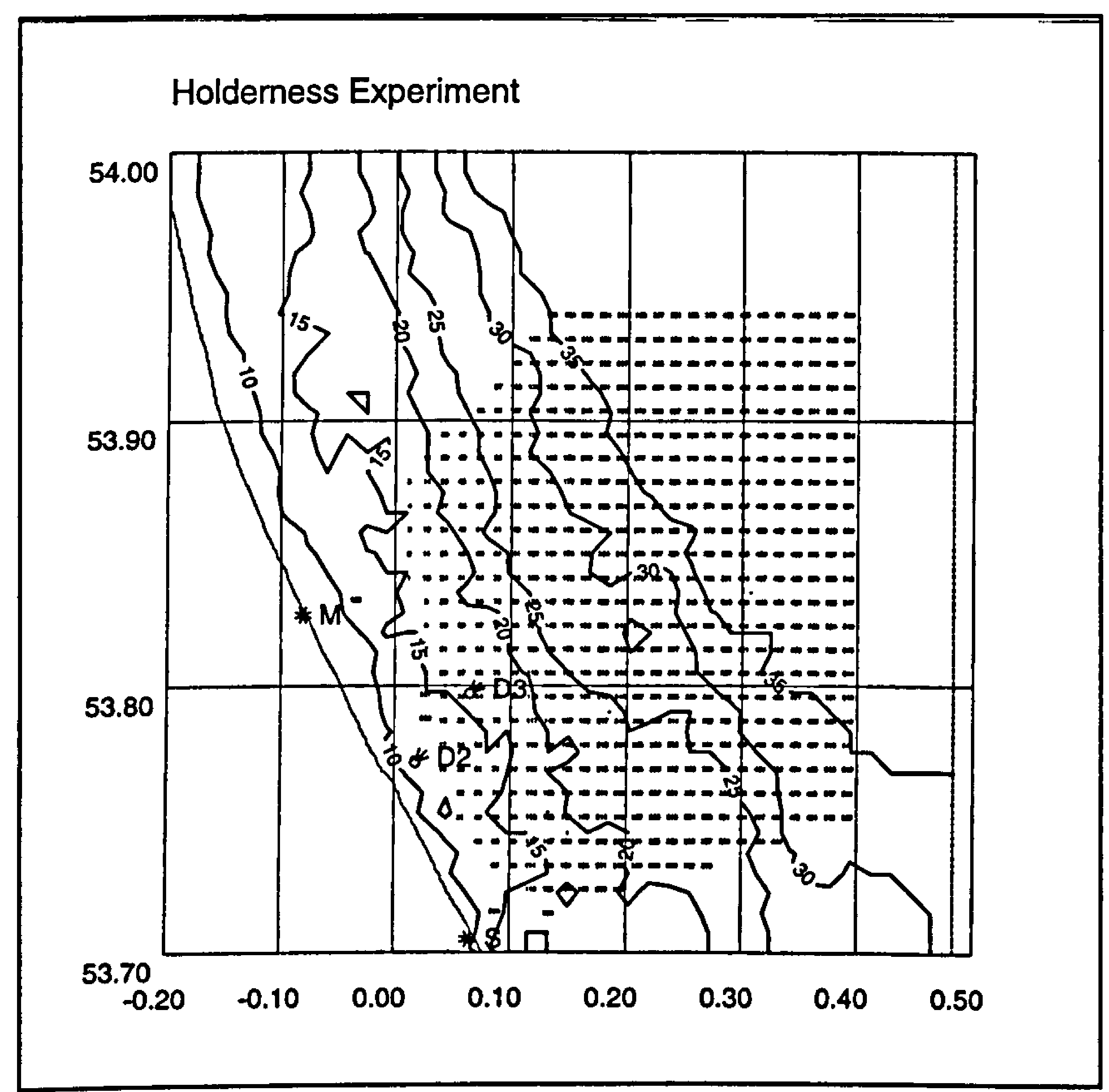

Figure 5.6: OSCR experiment. Map showing master $(\mathrm{M})$ and slave $(\mathrm{S})$ radar sites (*) and the two waveriders ( $\not{\varnothing})$.

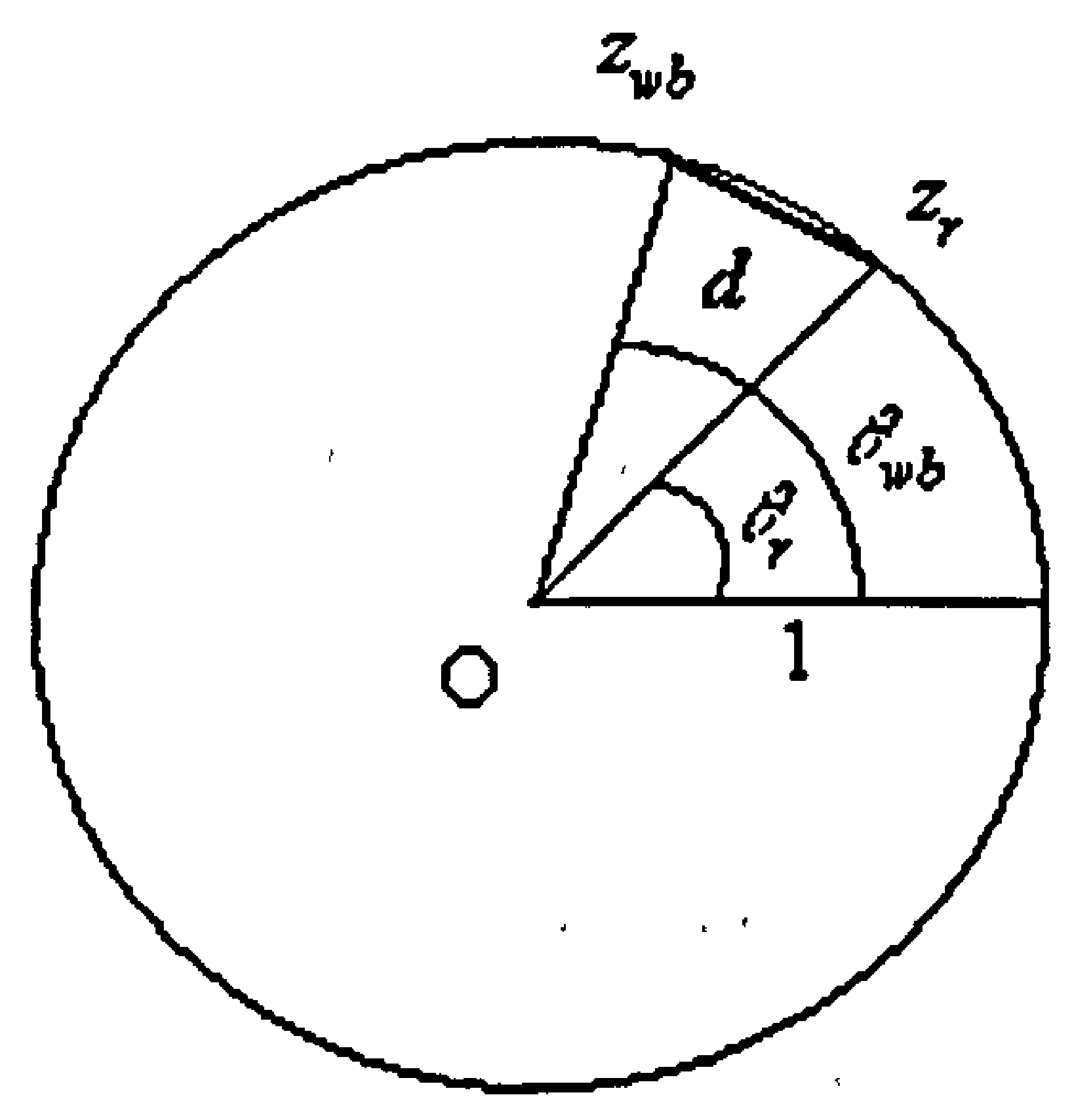

Figure 5.7: Diagram showing the distance error between the wave-buoy and radar measurements for the wind direction on the unit circle. 


\subsubsection{1 "Sea truth" consideration}

Let $\xi$ be the true wave parameter given by the wave buoy, $\xi \in\left\{T_{\omega}, H_{s}\right\}$ and let $\hat{\xi}$ be the wave parameter estimated by the different spectral techniques. $\xi$ and $\hat{\xi}$ are two vectors of dimension $M$.

For each wave parameter, a normalized global error vector, can be calculated:

$$
\beta=\left(\beta_{i}=\left[\frac{\xi_{i}-\hat{\xi}_{i}}{\xi_{i}}\right]^{2}\right)_{i=1 . . M}
$$

Thus, we can obtain an error mean, $\operatorname{Mean}(\beta)$, and an error variance, $\operatorname{Var}(\beta)$, given by:

$$
\begin{aligned}
& \operatorname{Mean}(\beta)=\frac{1}{M} \sum_{i=1}^{M} \beta_{i} \\
& \operatorname{Var}(\beta)=\frac{1}{M} \sum_{i=1}^{M}\left(\beta_{i}-\operatorname{Mean}(\beta)\right)^{2}
\end{aligned}
$$

These quantities can be considered as positive percentages. They will be evaluated for both spectral techniques in the following chapters.

\subsubsection{Symmetric regression}

This method of comparison makes the assumption that errors are present in both measurement systems (radar and buoy).

The directional waverider (Allender et al., 1989) can produce some error. The accuracy for the wave parameters $\left(H_{S}\right.$ and $T_{\omega}$ ) was demonstrated to be of $2.5-5 \%$ in the mean bias. Concerning the radar, this is still under investigation. However, we assume here that errors produced by both systems are of the same magnitude.

This statistical method was used by Wyatt and Ledgard (1997c) in order to make OSCR/directional waverider comparisons. This was applied to various parameters of the directional wave spectrum, not just $H_{S}$ and $T_{\omega}$. The results showed that the radar measurements were in general in very good agreement with the wave buoy measurements. 
The symmetric regression is in fact a Chi-square fitting and is based on a least-squares fit to a line with zero intercept (Press et al. 1992):

$$
\chi^{2}(\alpha)=\sum_{i=1}^{M} \frac{\left(y_{i}-\alpha x_{i}\right)^{2}}{\sigma_{y}^{2}+\alpha^{2} \sigma_{x}^{2}} \text { with } y=\alpha x,
$$

where $x=\left(x_{1} \ldots x_{M}\right)$ represents the wave buoy measurements , $y=\left(y_{1} \ldots y_{M}\right)$ the radar measurements, $\sigma_{x}$ and $\sigma_{y}$ are respectively the $x$ and $y$ standard deviations.

The denominator of equation (5.21) is obtained from:

$$
\operatorname{Var}\left(y_{i}-\alpha x_{i}\right)=\operatorname{Var}\left(y_{i}\right)+\alpha^{2} \operatorname{Var}\left(x_{i}\right)=\sigma_{y}^{2}+\alpha^{2} \sigma_{x}^{2} .
$$

Since errors are of the same magnitude for $x$ and $y$, we have $\sigma_{x}^{2}=\sigma_{y}^{2}$.

The minimization of (5.18) with respect to $\alpha$ gives two possible values:

$$
\begin{array}{ll}
\text { with } a=\sum_{i=1}^{M}\left(x_{i}^{2}-y_{i}^{2}\right) & \hat{\alpha}=\frac{a \pm \sqrt{\Delta}}{2 b}, \\
& , b=\sum_{i=1}^{M} x_{i} y_{i} \text { and } \Delta=a^{2}+4 b^{2} .
\end{array}
$$

Finally, the bias is obtained by taking the percentage variation from unity of the slope:

$$
\text { Bias }=1-\hat{\alpha}
$$

and the variance is estimated by : $\quad \operatorname{Var}=\frac{1}{M} \sum_{i=1}^{M}\left(\frac{y_{i}}{x_{i}}-\hat{\alpha}\right)^{2}$. 


\section{Chapter 6}

\section{Evaluation of the Modified Covariance Method with the OSCR system}

This chapter contains the results for the OSCR system obtained from the two methods of comparison described in the previous chapter: the Monte Carlo simulation study and the wave-buoy comparisons. These results concern the modified covariance method and the periodogram, the classical method used to get the Doppler spectrum. We compare these two spectral techniques in order to see if the modified covariance method, by using shorter data sets, can be an alternative method of producing the Doppler spectrum.

Also, these comparisons are used to determine the most suitable value of the model order $p$ for the modified covariance method.

In addition, we use spectral estimates generated by this method in the inversion procedure in order to give an overall view of the good quality of the directional spectra.

All these comparisons are done only on "good" quality periodogram suitable for extraction of the wave parameters. The cases of split Bragg peaks are not taken into account. 


\subsection{Good quality criteria}

This quality is tested on the presence of certain first- and second-order characteristics, in the Doppler Spectrum. In fact, we took the same criteria which have to be checked in order to proceed with the inversion procedure (Wyatt and Ledgard, 1997c):

1. The Doppler spectrum must contain a peak.

2. This peak must lie within a reasonable interval around the Bragg frequency in order to allow a maximum current of $2 \mathrm{~ms}^{-1}$.

3. The second-order contribution must be clearly defined: a non-zero number of frequencies in the ranges are required for inversion.

4. The signal-to-noise ratio in the second-order part of the spectrum must be greater than $15 \mathrm{~dB}$.

5. The peak in the second-order spectrum must be at least $3 \mathrm{~dB}$ less than the main first-order peak.

When these points are satisfied the three wave parameters mentioned in Chapter 5 , can be extracted and we can proceed with the different comparisons.

\subsection{Monte Carlo results}

The Monte Carlo simulation study consists of synthesising a signal whose spectral density function has similar characteristics to the observed signal (see chapter 5 section 5.1). We present the results obtained from three Doppler spectra. They correspond to three common sea-states:

- case a: when the wind is blowing predominantly away from the radar, that results in the negative (in frequency) Bragg peak being the greatest.

- case b: when the wind is blowing predominantly towards the radar, that results in the positive (in frequency) Bragg peak being the greatest.

- case c: when the wind direction is perpendicular to the radar beam, that results in the two Bragg peaks being equivalent.

Figures $6.1,6.2$ and 6.3 illustrate these three sea conditions respectively. 
PERIODOGRAM

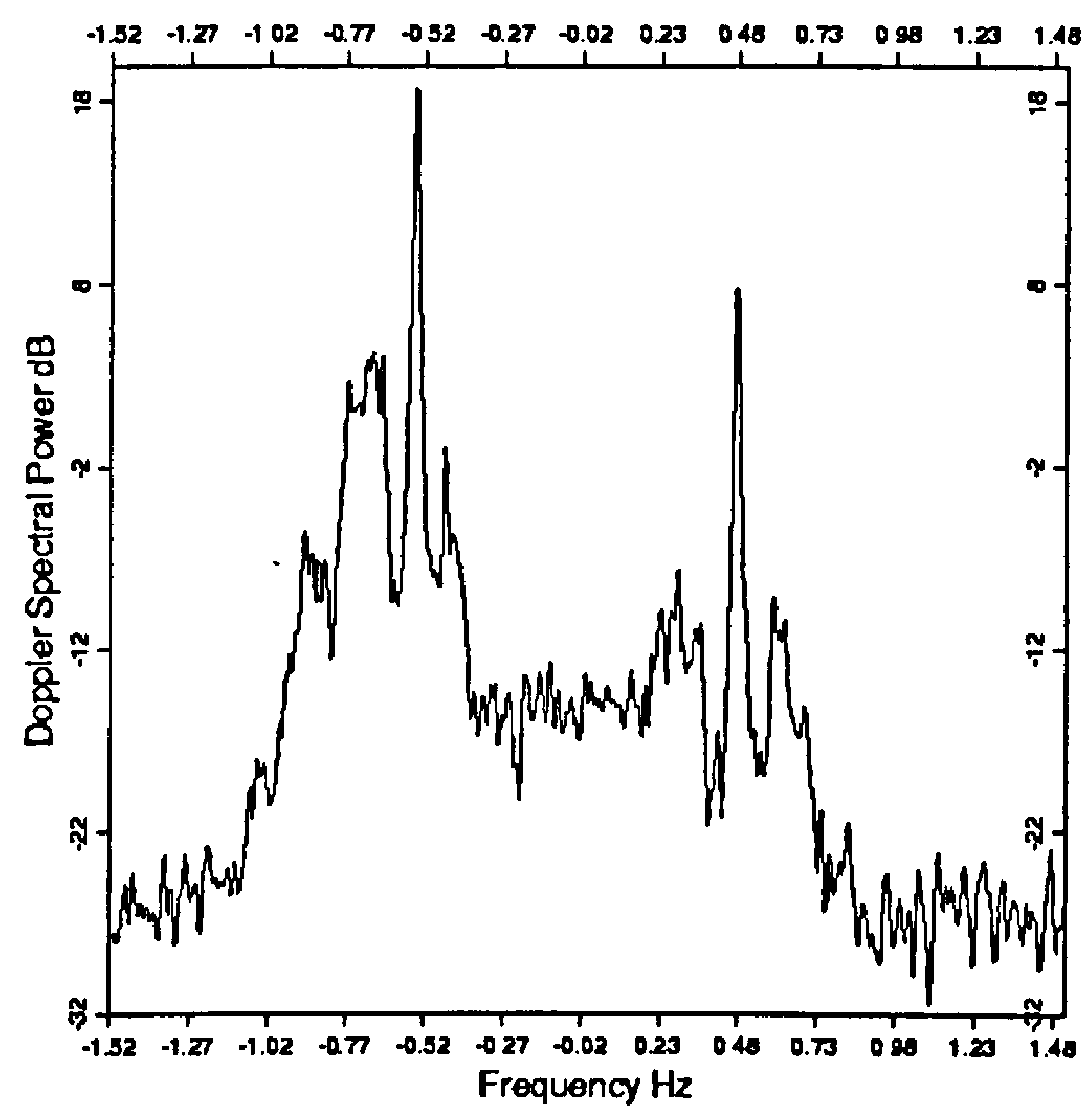

Figure 6.1: Doppler spectrum from the cell 144, measurement taken at 6:40pm on 1 January 1996, showing a wind blowing predominantly away from the radar, case (a).

PERIODOGRAM

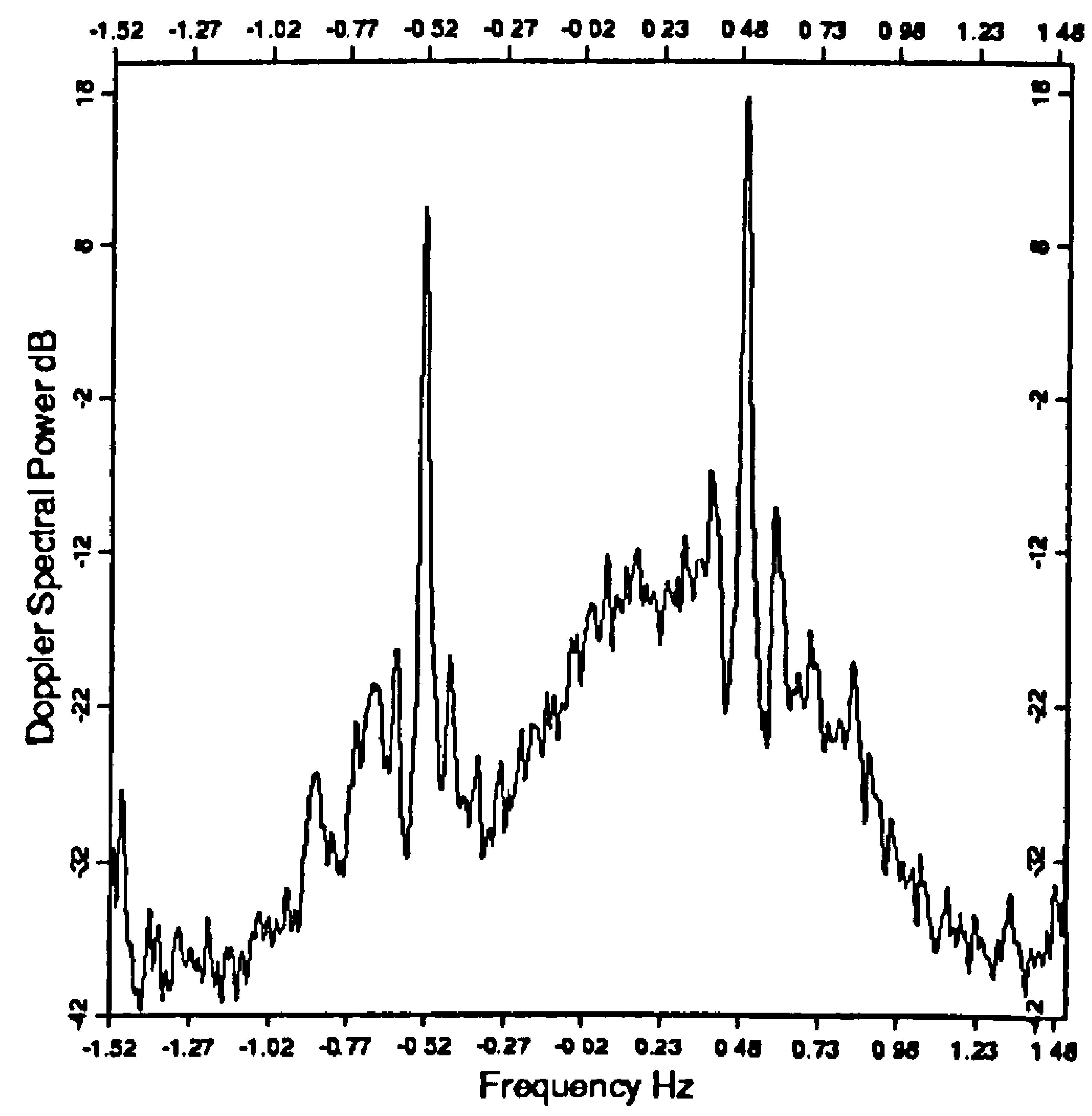

Figure 6.2: Doppler spectrum from the cell 79, measurement taken at 3pm on 27 December 1995, showing a wind blowing predominantly towards the radar, case (b). 


\section{PERIODOGRAM}

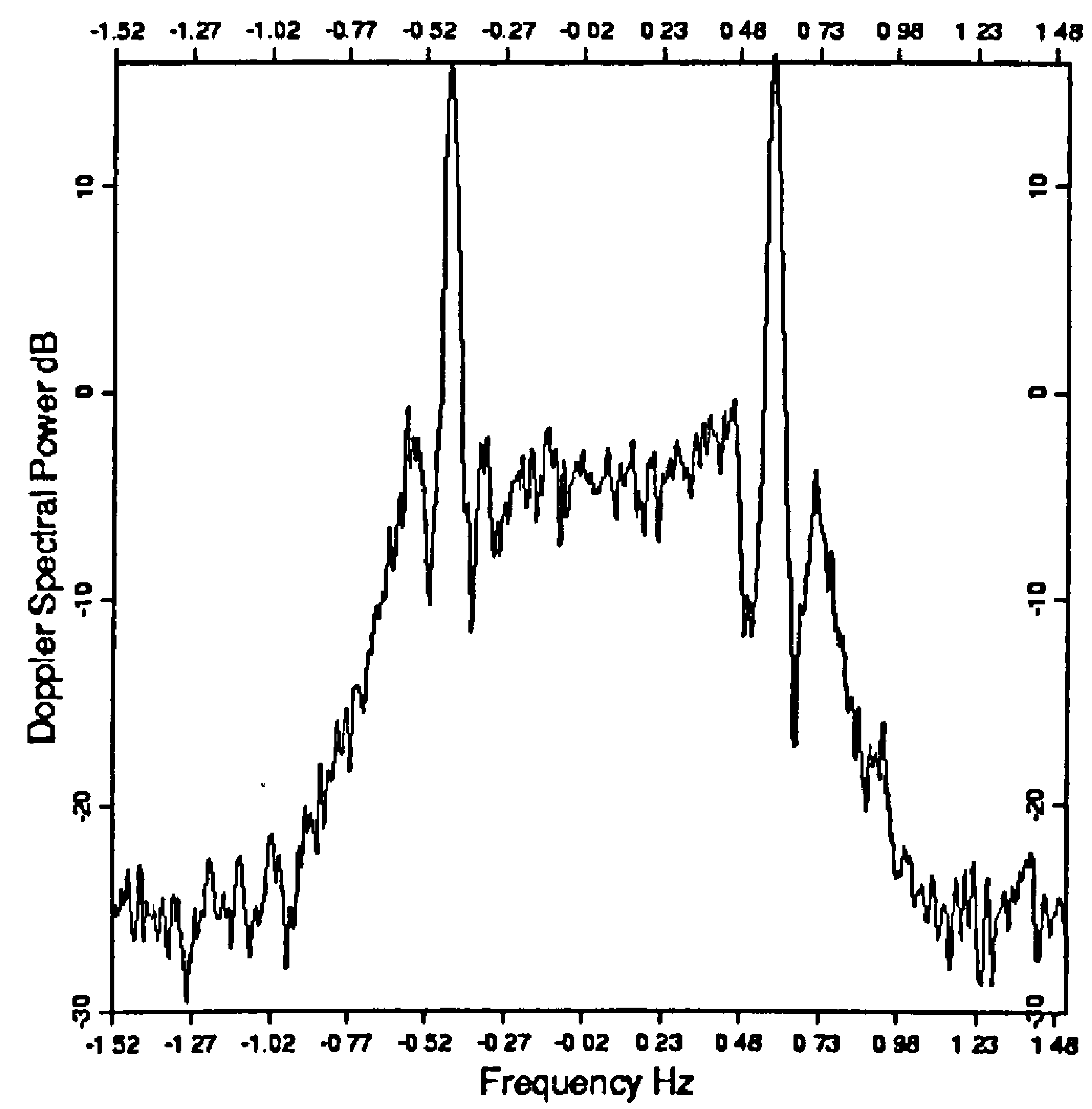

Figure 6.3: Doppler spectrum from the cell 220, measurement taken at 11:25am on 5 January 1996, showing a wind whose direction is perpendicular to the radar beam, case (c).

\subsubsection{Spectral estimates}

Table 6.1 shows the performances (see Chapter 5 section 5.1.2.1) of the periodogram and the modified covariance method (for different values of $p$ ) when they are applied to the periodogram in figure 6.1 (case (a)). These results are obtained from the differences that occured between the "true" spectrum (which is the periodogram) and the different spectral estimates. The four integrated error parameters defined in Chapter 5 are shown. As can be seen, the periodogram is better in terms of the given integrated parameters when 9 segments are averaged. However, when a single segment is used for the periodogram, all the AR PSD estimates are better. For example, when $p=40$, the linear IMSE is minimised for this method and when $p=20$, it is the logarithmic $\mathrm{IMSE}_{\log }$ which is minimised. 
Table 6.1

\begin{tabular}{|c|c|c|c|c|}
\hline \multirow{2}{*}{ Type } & \multicolumn{2}{|c|}{ Linear } & \multicolumn{2}{c|}{ Logarithmic } \\
\cline { 2 - 5 } & IVAR & IMSE & IVAR $_{\text {log }}$ & IMSE $_{\text {log }}$ \\
\hline $\begin{array}{c}\text { Periodogram } \\
\text { with 9 segments }\end{array}$ & 0.136 & 0.156 & 3.191 & 3.326 \\
\hline $\begin{array}{c}\text { Periodogram } \\
\text { with 1 segment }\end{array}$ & 7.668 & 8.4762 & 40.927 & 41.314 \\
\hline AR (p=20) & 1.255 & 2.151 & 9.113 & 10.394 \\
\hline AR $(p=30)$ & 1.015 & 1.523 & 10.581 & 11.959 \\
\hline AR $(p=40)$ & 1.004 & 1.335 & 11.143 & 12.46 \\
\hline AR $(p=50)$ & 1.195 & 1.429 & 12.123 & 12.741 \\
\hline
\end{tabular}

Comparison of performance of the modified covariance method and the periodogram. Four different model orders are taken for the modified covariance method. Results obtained from the cell 144 (measurement taken at 6:40pm on 1 January 1996), case (a).

Table 6.2

\begin{tabular}{|c|c|c|c|c|}
\hline \multirow{2}{*}{ Type } & \multicolumn{2}{|c|}{ Linear } & \multicolumn{2}{c|}{ Logarithmic } \\
\cline { 2 - 5 } & IVAR & IMSE & IVAR $_{\text {log }}$ & IMSE $_{\text {log }}$ \\
\hline $\begin{array}{c}\text { Periodogram } \\
\text { with 9 segments }\end{array}$ & 0.13 & 0.16 & 0.009 & 0.01 \\
\hline $\begin{array}{c}\text { Periodogram } \\
\text { with 1 segment }\end{array}$ & 8.41 & 9.12 & 0.11 & 0.114 \\
\hline $\mathrm{AR}(p=20)$ & 11.25 & 30.23 & 0.036 & 0.064 \\
\hline $\mathrm{AR}(p=30)$ & 10.83 & 21.97 & 0.044 & 0.064 \\
\hline $\mathrm{AR}(p=40)$ & 1.9 & 2.56 & 0.04 & 0.05 \\
\hline $\mathrm{AR}(p=50)$ & 2.12 & 2.61 & 0.052 & 0.057 \\
\hline
\end{tabular}

Comparison of performance of the modified covariance method and the periodogram.

Two different model orders are taken for the modified covariance method. Results obtained from the cell 79 (measurement taken at 3pm on 27 December 1995), case (b). 
Table 6.3

\begin{tabular}{|c|c|c|c|c|}
\hline \multirow{2}{*}{ Type } & \multicolumn{2}{|c|}{ Linear } & \multicolumn{2}{c|}{ Logarithmic } \\
\cline { 2 - 5 } & IVAR & IMSE & IVAR $_{\log }$ & IMSE $_{\text {log }}$ \\
\hline $\begin{array}{c}\text { Periodogram } \\
\text { with 9 segments }\end{array}$ & 0.099 & 0.104 & 0.123 & 0.135 \\
\hline $\begin{array}{c}\text { Periodogram } \\
\text { with 1 segment }\end{array}$ & 1.556 & 1.61 & 1.395 & 1.605 \\
\hline AR $(p=20)$ & 0.224 & 0.778 & 0.163 & 0.37 \\
\hline AR $(p=30)$ & 0.164 & 0.273 & 0.203 & 0.334 \\
\hline AR $(p=40)$ & 0.349 & 0.44 & 0.284 & 0.304 \\
\hline AR $(p=50)$ & 0.397 & 0.469 & 0.325 & 0.347 \\
\hline
\end{tabular}

Comparison of performance of the modified covariance method and the periodogram.

Two different model orders are taken for the modified covariance method.

Results obtained from the cell 220 (measurement taken at 11:25am on 5 January 1996), case (c).

The performances concerning the other two cases (b) and (c) are shown in tables 6.2 and 6.3 respectively. The comments are the same, except for the modified covariance method from which the results depend on the model order $p$. For case (b), the IMSE and IMSE $_{\log }$ are minimised when $p=40$ and for case (c), these error parameters are minimised for $p=30$.

As you can notice, the error parameters for the three different cases are not of the same order. This is due to the variations present in the second-order contribution and also to the choice of the distance (linear or logarithmic). For cases (a) and (b), the linear error parameters are comparable except for two $p$-values with the modified covariance method (table 6.2, $p=20$ and 30 ) showing large errors. However, concerning the logarithmic parameters, the errors are much larger for case (a). This can be explained by the presence of larger variations in amplitude of the second-order contribution of the periodogram shown in figure 6.1, which can induce more error in this case (a). Since the variations of the second-order part of the spectrum for case (b) (see figure 6.2) are small, the logarithmic errors are quite small. Concerning the last case (c), the linear and logarithmic errors are of the same magnitude since the variations of the second-order spectrum are small. 


\subsubsection{Wave parameters}

The three following tables 6.4, 6.5 and 6.6 contain the error measurements obtained from three ocean wave parameters (see Chapter 5 section 5.1.2.2), respectively for each sea state (a), (b) and (c).

As for the previous comparison with the spectral estimates, the periodogram is better in terms of the given parameters when 9 segments are used and worse when a single segment is used. For certain values of $p$, the modified covariance method is comparable to the 9-segment periodogram in terms of the bias. For example, in table 6.4, when $p=40$, the bias for $H_{S}$ and $T_{\omega}$ is minimized for this method. And this happens for the two other cases (tables 6.5 and 6.6) when $p=30$. For the last case (table 6.6), in comparison with the periodogram with 9 segments, less error is obtained for $T_{\omega}$ for the modified covariance method when $p=30$.

In contrary to the comparisons concerning the spectral estimates, the error parameters are of the same magnitude for the three cases. The variations of the second-order spectrum have no impact in these errors since the wave parameters are calculated from integrals of this contribution.

Table 6.4

\begin{tabular}{|c|c|c|c|c|c|c|}
\hline \multirow{2}{*}{ Type } & \multicolumn{2}{|c|}{$H_{S}$} & \multicolumn{2}{c|}{$T_{\omega}$} & \multicolumn{2}{c|}{$\theta_{\omega}$} \\
\cline { 2 - 7 } & Bias & Variance & Bias & Variance & Bias & Variance \\
\hline $\begin{array}{c}\text { Periodogram } \\
\text { with 9 segments }\end{array}$ & 0.0388 & 0.0352 & 0.0297 & 0.0253 & 0.004 & 0.006 \\
\hline $\begin{array}{c}\text { Periodogram } \\
\text { with 1 segment }\end{array}$ & 0.489 & 0.6216 & 0.3978 & 0.3441 & 0.022 & 0.0646 \\
\hline $\operatorname{AR}(p=20)$ & -0.0456 & 0.0841 & -0.1102 & 0.0154 & 0.0046 & 0.026 \\
\hline $\operatorname{AR}(p=30)$ & 0.0018 & 0.099 & -0.0588 & 0.0105 & 0.006 & 0.0298 \\
\hline $\operatorname{AR}(p=40)$ & 0.0017 & 0.0928 & -0.039 & 0.0062 & 0.0111 & 0.0286 \\
\hline $\operatorname{AR}(p=50)$ & 0.0855 & 0.2019 & 0.1372 & 0.4085 & 0.017 & 0.0315 \\
\hline
\end{tabular}

Comparison of performance of the modified covariance method and the periodogram.

Four different model orders are taken for the modified covariance method. Results obtained from the cell 144 (measurement taken at 6:40pm on 1 January 1996), case (a). 
Table 6.5

\begin{tabular}{|c|c|c|c|c|c|c|}
\hline \multirow{2}{*}{ Type } & \multicolumn{2}{|c|}{$H_{S}$} & \multicolumn{2}{c|}{$T_{\omega}$} & \multicolumn{2}{c|}{$\theta_{\omega}$} \\
\cline { 2 - 7 } & Bias & Variance & Bias & Variance & Bias & Variance \\
\hline $\begin{array}{c}\text { Periodogram } \\
\text { with 9 segments }\end{array}$ & 0.0265 & 0.0333 & -0.0048 & 0.0254 & -0.0004 & 0.002 \\
\hline $\begin{array}{c}\text { Periodogram } \\
\text { with 1 segment }\end{array}$ & 0.689 & 2.082 & 0.666 & 2.109 & -0.006 & 0.02 \\
\hline AR $(p=20)$ & -0.29 & 0.159 & -0.44 & 0.0304 & -0.01 & 0.173 \\
\hline AR $(p=30)$ & -0.0328 & 0.2309 & -0.1579 & 0.0278 & -0.0163 & 0.0183 \\
\hline AR $(p=40)$ & 0.229 & 1.235 & 0.497 & 3.523 & -0.0049 & 0.0151 \\
\hline AR $(p=50)$ & 0.751 & 2.513 & 1.593 & 8.004 & -0.01 & 0.021 \\
\hline
\end{tabular}

Comparison of performance of the modified covariance method and the periodogram.

Two different model orders are taken for the modified covariance method.

Results obtained from the cell 79 (measurement taken at 3pm on 27 December 1995), case (b).

Table 6.6

\begin{tabular}{|c|c|c|c|c|c|c|}
\hline \multirow{2}{*}{ Type } & \multicolumn{2}{|c|}{$H_{S}$} & \multicolumn{2}{c|}{$T_{\omega}$} & \multicolumn{2}{c|}{$\theta_{\omega}$} \\
\cline { 2 - 7 } & Bias & Variance & Bias & Variance & Bias & Variance \\
\hline $\begin{array}{c}\text { Periodogram } \\
\text { with 9 segments }\end{array}$ & 0.0795 & 0.1927 & 0.2786 & 0.6285 & 0.005 & 0.0053 \\
\hline $\begin{array}{c}\text { Periodogram } \\
\text { with 1 segment }\end{array}$ & 0.5835 & 0.5643 & 1.2864 & 1.4424 & 0.002 & 0.0285 \\
\hline $\operatorname{AR~}(p=20)$ & -0.2608 & 0.0548 & -0.1479 & 0.0634 & -0.009 & 0.0076 \\
\hline $\operatorname{AR}(p=30)$ & -0.1732 & 0.0516 & 0.03 & 0.1776 & -0.0069 & 0.0078 \\
\hline $\operatorname{AR}(p=40)$ & 0.4357 & 0.5211 & 1.1652 & 2.7414 & -0.021 & 0.012 \\
\hline $\operatorname{AR}(p=50)$ & 0.4099 & 0.4776 & 1.0059 & 2.0099 & 0.0105 & 0.0129 \\
\hline
\end{tabular}

Comparison of performance of the modified covariance method and the periodogram.

Two different model orders are taken for the modified covariance method. Results obtained from the cell 220 (measurement taken at 11:25am on 5 January 1996), case (c).

\subsubsection{Summary}

15 more Monte Carlo simulations were carried out which revealed that for the modified covariance method, the different error parameters are generally minimized 
for $20 \leq p \leq 40$; that helps us in the selection of the model order $p$. This range for $p$ is suitable for $N=512$ samples. Nevertheless, given that the difference between 512 and 896 points is not large and since no relation exists between $p$ and $N$, we can also apply this range for $N=896$ samples.

For the different sea-states considered (seen in figures 6.1, 6.2 and 6.3), the superpositions of the periodogram and the AR PSD estimate (given by the modified covariance method) with the appropriate model order $p$ given by the Monte Carlo comparison are shown in figures $6.4,6.5$ and 6.6 respectively. As you can see, their similarity is quite good. In the next section, we propose a general $p$-value taken in this range.

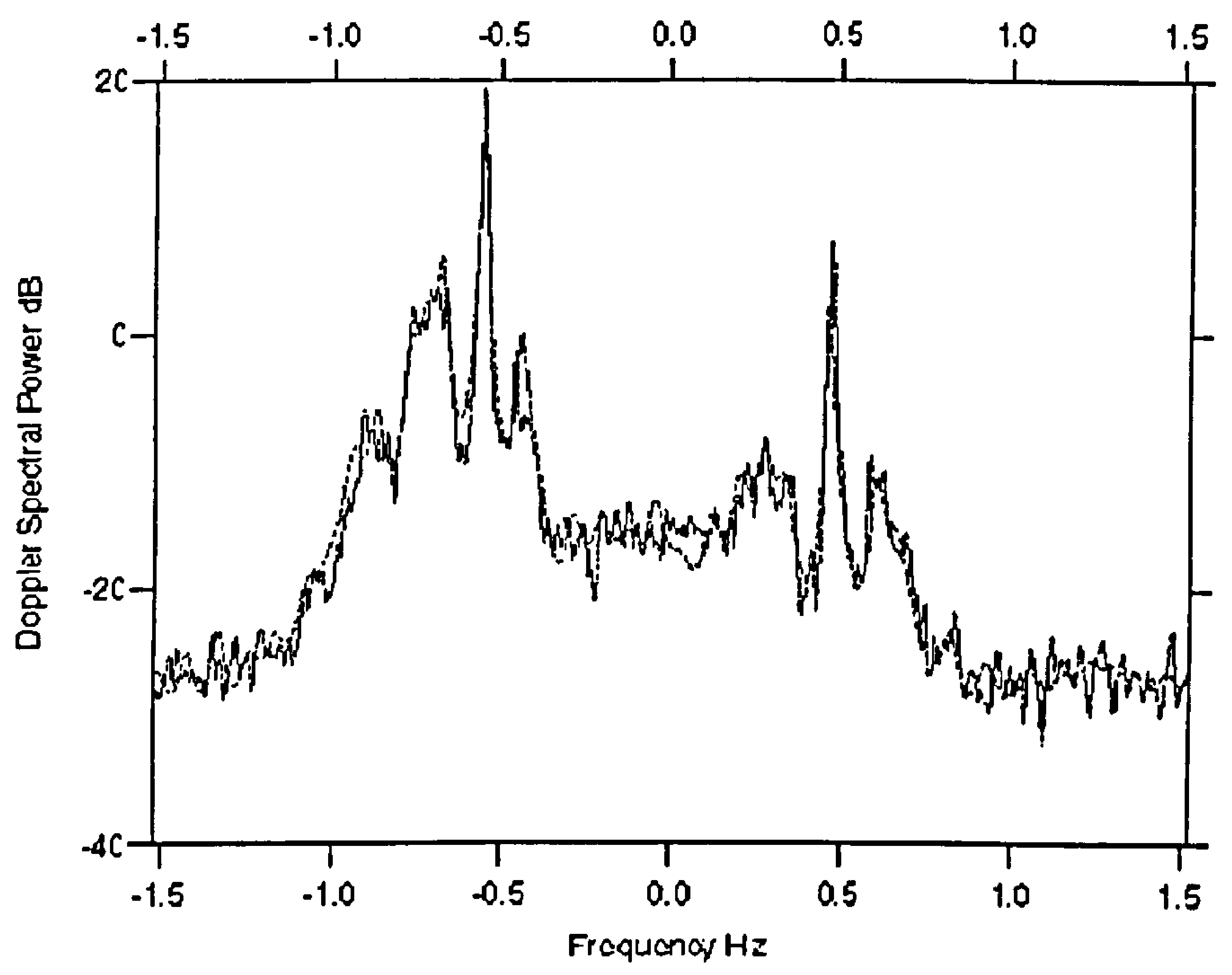

Figure 6.4: Superposition of the periodogram and the AR PSD estimate of the OSCR file cell 144 (dotted line) with $N=896$ and $p=40$. 


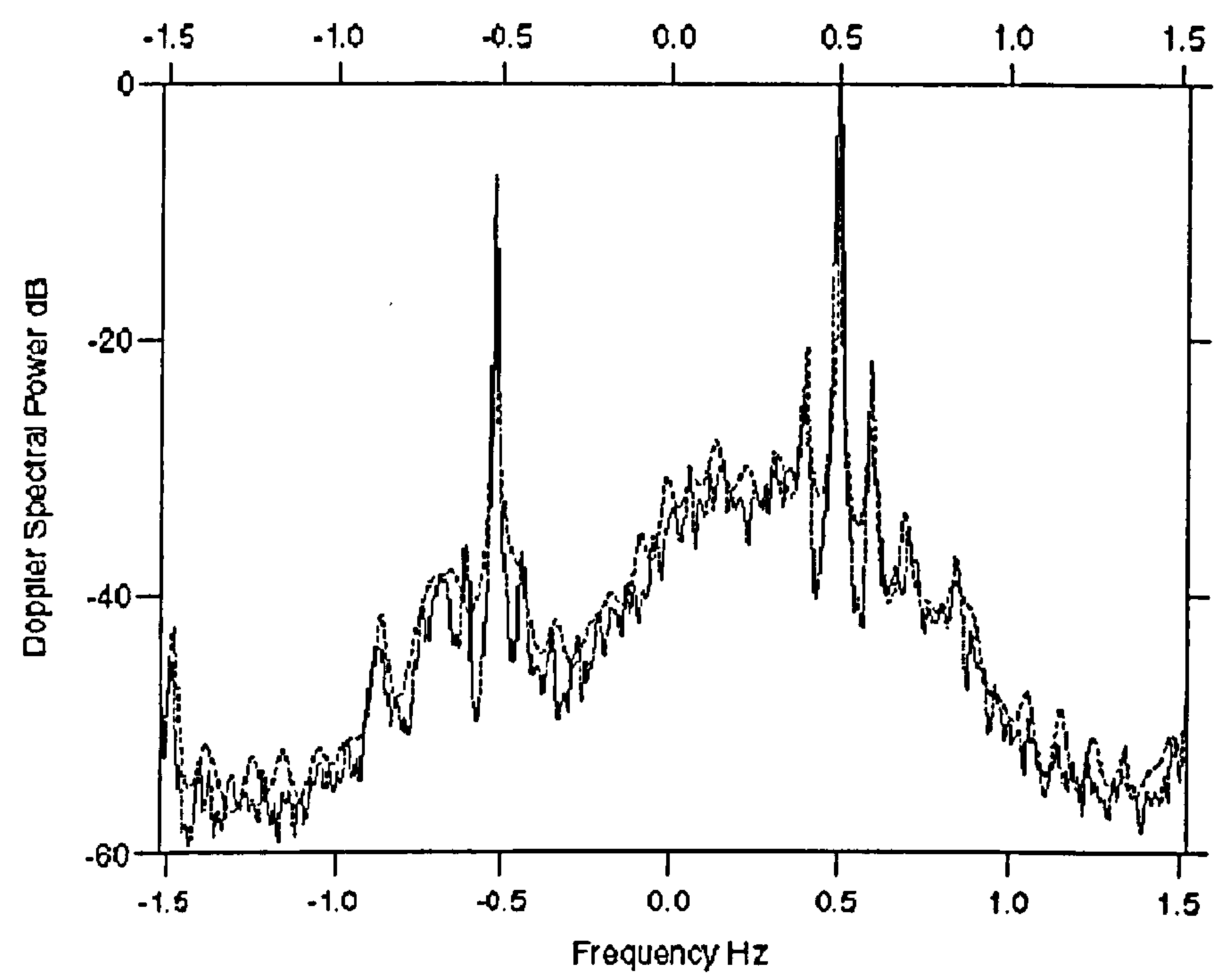

Figure 6.5: Superposition of the periodogram and the AR PSD estimate of the OSCR file cell 79 (dotted line) with $N=896$ and $p=40$.

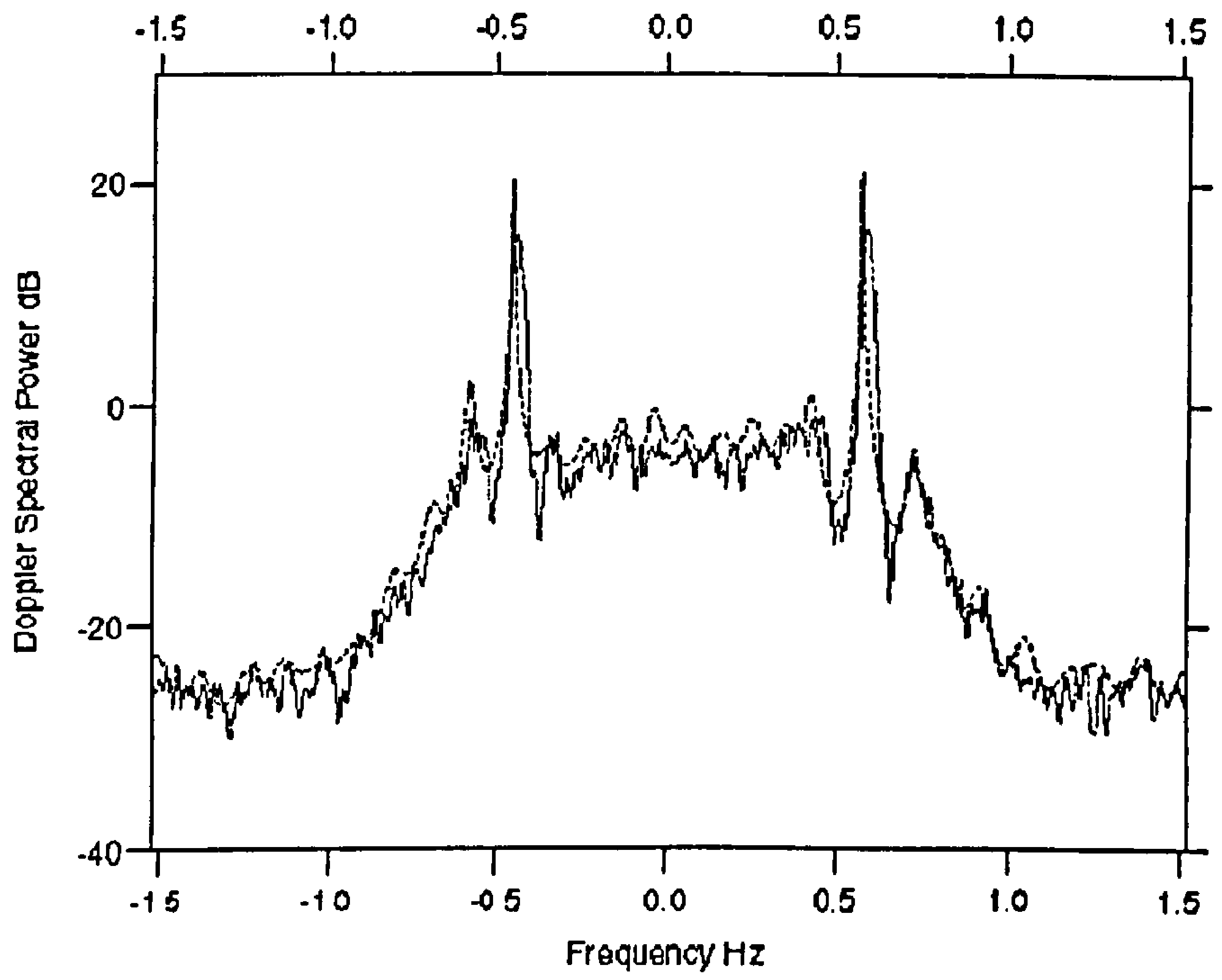

Figure 6.6: Superposition of the periodogram and the AR PSD estimate of the OSCR file cell 220 (dotted line) with $N=896$ and $p=30$. 


\subsection{Wave-buoy results}

The different histograms shown in figures 6.7, 6.8, 6.9, 6.10, and the tables 6.7 and 6.8, give the results obtained from the wave-buoy comparison (see Chapter 5 section 5.2). The bias and variance, relative to the wave-buoy measurements, are determined with the wave parameters extracted from the different spectral estimates. We used only good quality radar measurements (master and slave), obtained from the cell 55 and dated from 4 until 11 January 1996. This quality was discussed in section 6.1. We considered each measurement to be independent. In total, 105 measurements were used to give the results. Concerning the periodogram, we took the full data set: 2688 samples.

\subsubsection{Wind direction, $\theta_{\omega}$}

Table 6.7 contains the mean difference and the standard deviation obtained for $\theta_{\omega}$ (see Chapter 5 section 5.2.2) with the periodogram with full and short data sets and the modified covariance method with different order models. As it can be seen, the results are comparable for both spectral techniques. The modified covariance method gives less error in terms of the given parameters when $p=30$.

Also, this table shows the errors resulting from the modified covariance method when it is applied to the three successive 5-minute measurements. As can be seen, both differences remain nearly constant during this hour measurement. 
Table 6.7

\begin{tabular}{|c|c|c|}
\hline \multirow{2}{*}{ Type } & \multicolumn{2}{|c|}{$\theta_{\omega}\left(^{\circ}\right)$} \\
\cline { 2 - 3 } & mean difference & standard deviation \\
\hline $\begin{array}{c}\text { Periodogram } \\
\text { full data set }\end{array}$ & 16.55 & 12.52 \\
\hline $\begin{array}{c}\text { Periodogram } \\
\text { second collection }\end{array}$ & 17.16 & 14.53 \\
\hline $\begin{array}{c}\text { AR(20) } \\
\text { second collection }\end{array}$ & 16.8 & 12.15 \\
\hline $\begin{array}{c}\text { AR(30) } \\
\text { second collection }\end{array}$ & 16.26 & 11.5 \\
\hline $\begin{array}{c}\text { AR(40) } \\
\text { second collection }\end{array}$ & 16.34 & 11.78 \\
\hline $\begin{array}{c}\text { AR(30) } \\
\text { first collection }\end{array}$ & 17.59 & 13.18 \\
\hline $\begin{array}{c}\text { AR(30) } \\
\text { third collection }\end{array}$ & 17.3 & 14.38 \\
\hline
\end{tabular}

Errors resulting from the comparison between the wave-buoy measurements and the wave parameter $\theta_{\omega}$ extracted from the periodogram with full and short data sets and the modified covariance method with three different model orders.

\subsection{2 "Sea truth" consideration}

In this section the wave-buoy measurements are considered as "sea truth" measurements (see Chapter 5 section 5.2.1).

Table 6.8 shows the error parameters resulted from the modified covariance method with three different model orders $p$. They were taken in the range given by the Monte Carlo simulation study $(20 \leq p \leq 40)$. We used the second 5-minute collection since this is closer to the wave-buoy measurements in time. As can be seen for $H_{S}$ and $T_{\omega}$, as for $\theta_{\omega}$, lower errors are obtained when $p=30$. Therefore in the future we will be using this model order. We added to this table the results obtained from the periodogram with the same amount of data (896 samples). These errors are quite large, showing the efficiency of the modified covariance method when using short data sets.

Figures 6.7 and 6.8 show the error parameters resulting from the periodogram with 
full data set and the modified covariance method with 896 samples. In general, the error mean is less than $6 \%$ for $H_{S}, 3 \%$ for $T_{\omega}$ and the error variance is less than $1.2 \%$ for $H_{S}, 0.2 \%$ for $T_{\omega}$. These results obtained for the error variance give a very good accuracy for the different wave parameters, given that the directional waverider can produce $2.5-5 \%$ error (Allender et al. 1989) in the bias.

The periodogram is better in terms of the mean, that is not the case for the variance which shows less error for the modified covariance method.

Table 6.8

\begin{tabular}{|c|c|c|c|c|}
\hline \multirow{2}{*}{ Type } & \multicolumn{2}{|c|}{$H_{S}(\%)$} & \multicolumn{2}{c|}{$T_{\omega}(\%)$} \\
\cline { 2 - 5 } & Bias & Variance & Bias & Variance \\
\hline AR $(p=20)$ & 10 & 3.9 & 3.7 & 0.2 \\
\hline AR $(p=30)$ & 5.9 & 1.2 & 2.5 & 0.1 \\
\hline AR $(p=40)$ & 10.9 & 16.9 & 29.9 & 638.8 \\
\hline Periodogram & 29.4 & 418.8 & 62.5 & 686.8 \\
\hline
\end{tabular}

Errors resulting from the comparison between the wave-buoy measurements and the wave parameters ( $H_{S}$ and $T_{\omega}$ ) extracted from the modified covariance method with three different model orders and the periodogram with short data sets.

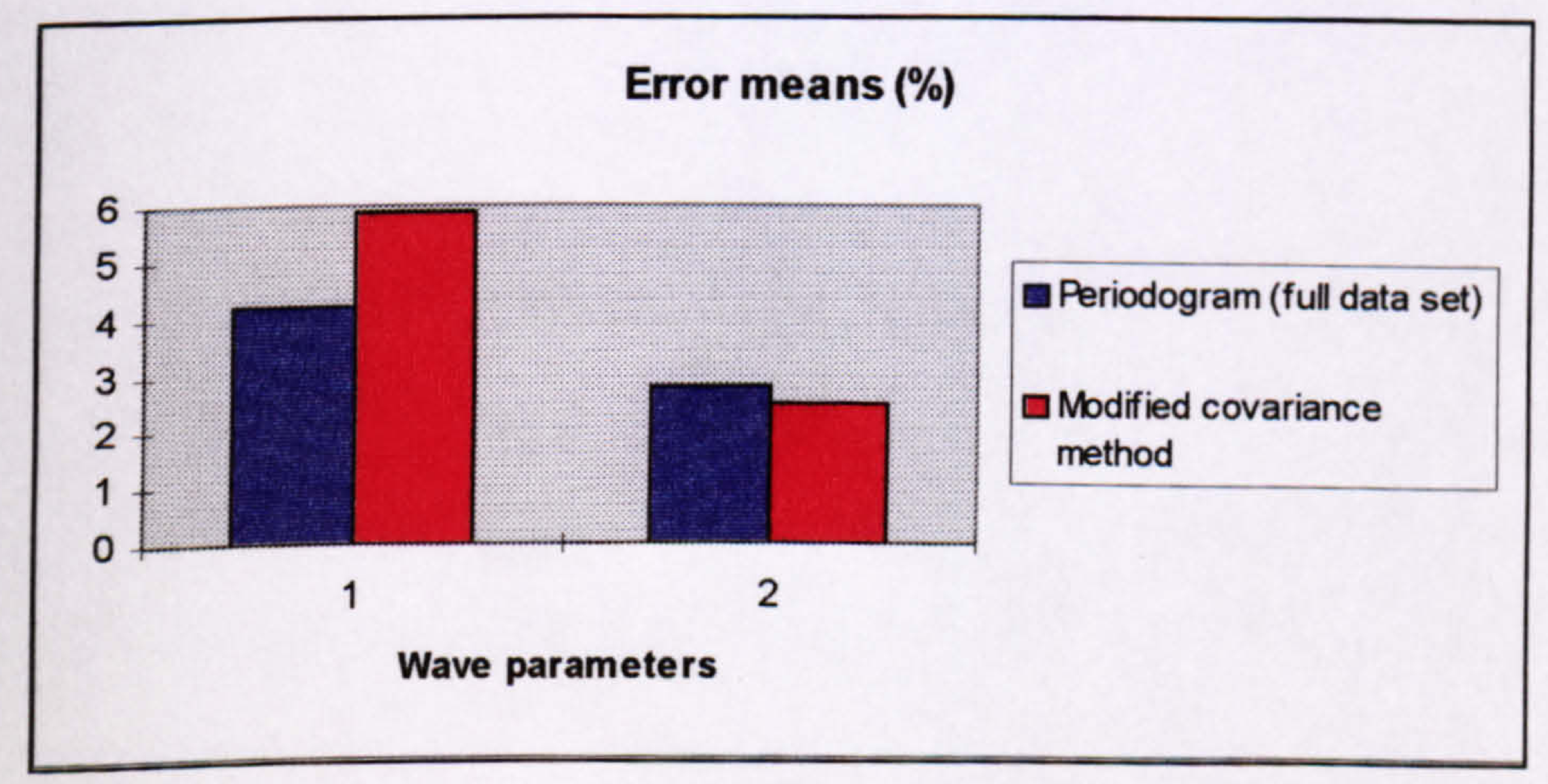

Figure 6.7: Histogram showing the mean of the error resulting from the comparison between the wave-buoy measurements and the wave parameters $\left(H_{S}\right.$ and $\left.T_{\omega}\right)$ extracted from the periodogram with full data set and the modified covariance method with 896 samples and $p=30$. On the $x$-axis, the numbers 1 and 2 represent $H_{S}$ and $T_{\omega}$ respectively. 


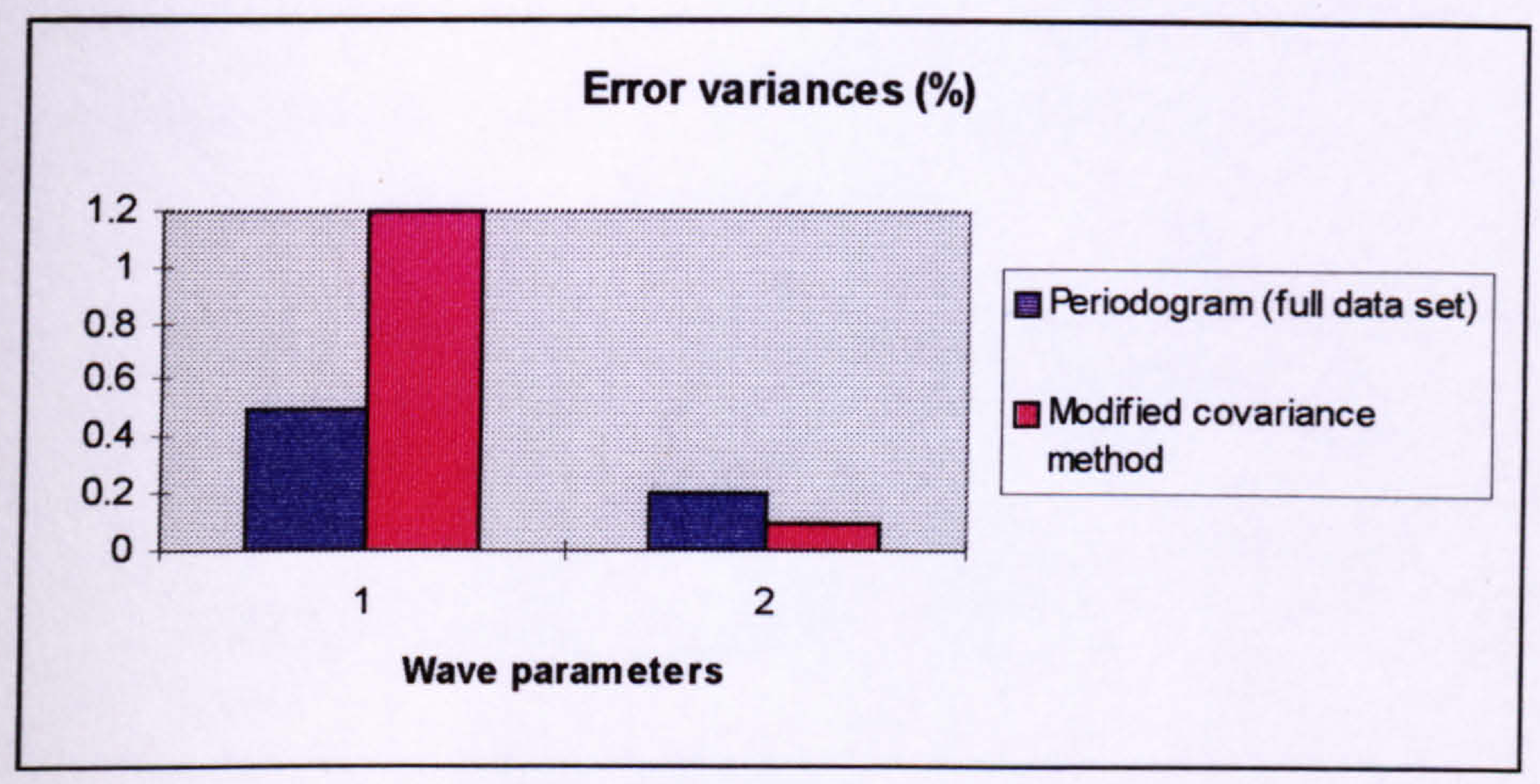

Figure 6.8: Histogram showing the variance of the error resulting from the comparison between the wave-buoy measurements and the wave parameters $\left(H_{S}\right.$ and $T_{\omega}$ ) extracted from the periodogram and the modified covariance method with 896 samples and $p=30$. On the $x$-axis, the numbers 1 and 2 represent $H_{S}$ and $T_{\omega}$ respectively.

The next two figures 6.9 and 6.10 show the errors resulting from the modified covariance method when it is applied to the three successive 5-minute measurements. As can be seen, both bias and variance remain nearly constant during this hour measurement particularly for $T_{\omega}$. Concerning $H_{S}$ (which is more sensitive to error) the last collection reveals more difference. However, one can say that the data remain reasonably stationary during the hour measurement.

From all the results obtained for $H_{S}$ and $T_{\omega}$, we can notice their degree of sensitivity. The largest errors concern $H_{S}$. The difference between $H_{S}$ and $T_{\omega}$ comes from the facts that $H_{S}$ involves the first-order spectrum since the second-order contribution is normalized with respect to the first-order spectrum (see Chapter 2, sections 2.4.1 and 2.4.2). 


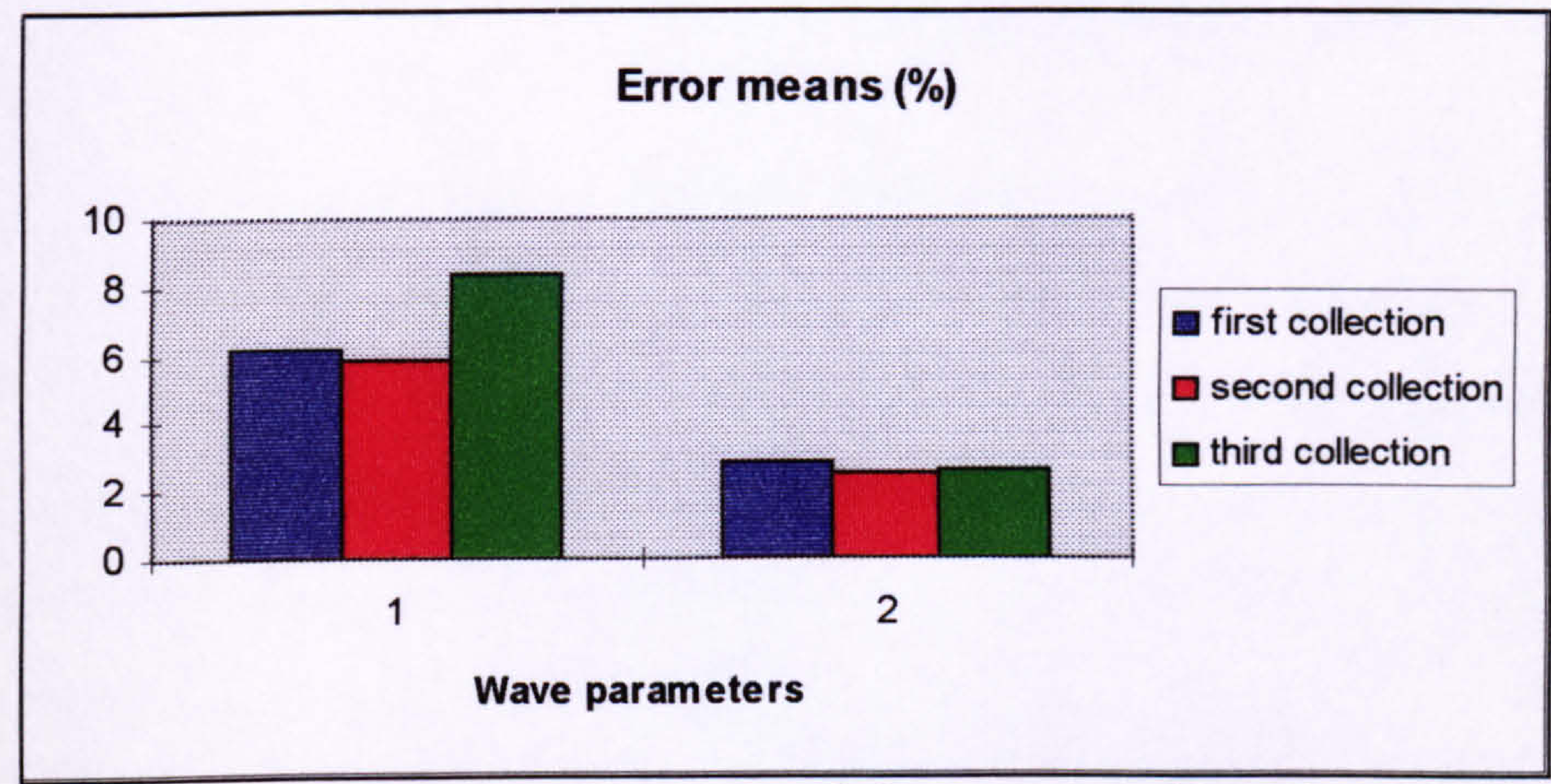

Figure 6.9: Histogram showing the mean of the error resulting from the comparison between the wave-buoy measurements and the wave parameters ( $H_{S}$ and $T_{\omega}$ ) extracted from the modified covariance method over the three collections of 896 samples with $p=30$. On the $x$-axis, the numbers 1 and 2 represent $H_{S}$ and $T_{\omega}$ respectively.

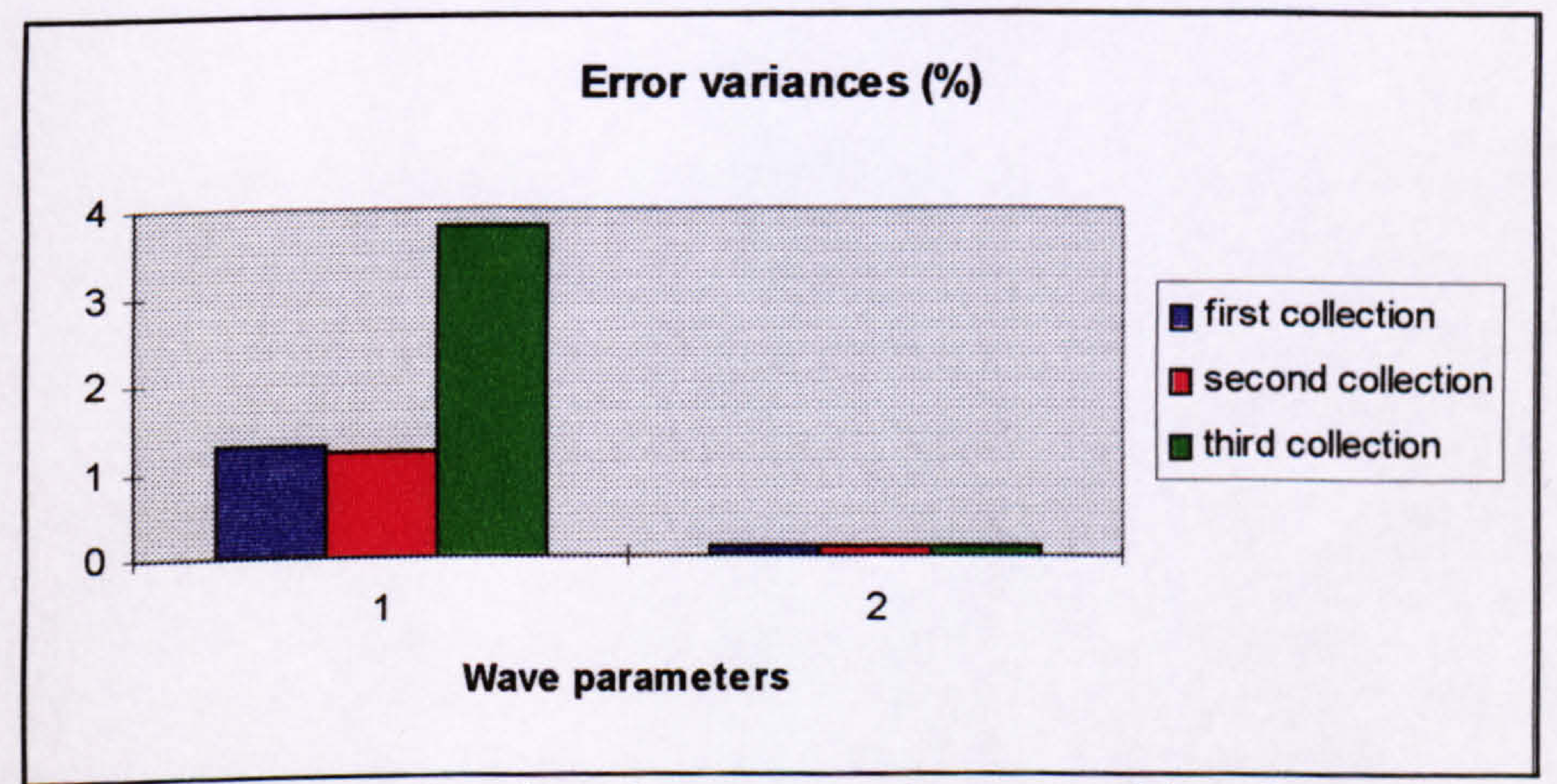

Figure 6.10: Histogram showing the error variance resulting from the comparison between the wave-buoy measurements and the wave parameters ( $H_{S}$ and $T_{\omega}$ ) extracted from the modified covariance method over the three collections of 896 samples with $p=30$. On the $x$-axis, the numbers 1 and 2 represent $H_{S}$ and $T_{\omega}$ respectively. 


\subsubsection{Symmetric regression}

This comparison assumes that errors can be produced in both measurements (see Chapter 5 section 5.2.2).

Table 6.9 shows the results obtained from the periodogram with full data set ( $N=2688$ ) and the modified covariance method with $N=896$ and the recommended model order $p=30$. As can be seen, the periodogram and the modified covariance method have equal but different advantages. The periodogram gives better results in terms of the variance for $H_{S}$ and bias for $T_{\omega}$ and the modified covariance method gives better results in terms of the bias for $H_{S}$ and variance for $T_{\omega}$.

We can notice that these errors are larger than the ones obtained with the sea truth consideration. This may be due to the fact that errors from both system build up.

However, they are of the same order as the results obtained by Wyatt and Ledgard (1997c), showing good agreement between these results and theirs.

\section{Table 6.9}

\begin{tabular}{|c|c|c|c|c|}
\hline \multirow{2}{*}{ Type } & \multicolumn{2}{|c|}{$H_{S}(\%)$} & \multicolumn{2}{c|}{$T_{\omega}(\%)$} \\
\cline { 2 - 5 } & Bias & Variance & Bias & Variance \\
\hline Periodogram & 6.5 & 4.2 & -2.5 & 2.7 \\
\hline AR $(p=30)$ & 5.9 & 5.8 & 6 & 2.2 \\
\hline
\end{tabular}

Errors resulting from the symmetric regression between the wave-buoy measurements and the wave parameters ( $H_{S}$ and $T_{\omega}$ )extracted from the periodogram with full data set and the modified covariance method. 


\subsection{Conclusion}

Since we are in the ideal situation for the periodogram (no case of split peaks are considered), these results showed that the modified covariance method and the periodogram are comparable.

With the Monte Carlo simulation study, the results obtained by the modified covariance method with shorter data sets are quite good, particularly for the wave measurements. Also, this study allows us to solve the problem of selection of the model order $p$ for the modified covariance method. For the OSCR system, with $N=896$, we must take $20 \leq p \leq 40$.

With the wave-buoy comparisons, a more precise value for $p$ is obtained. Less error resulting from the "sea-truth" for the modified covariance method is obtained when $p=30$. The results show that the modified covariance method is better for $T_{\omega}$ and $\theta_{\omega}$. The symmetric regression shows good agreement between radar measurements (obtained from the periodogram and also from the modified covariance method) and wave-buoy measurements.

Finally, the Monte Carlo method and the wave-buoy comparisons show the efficiency of the modified covariance method when short data sets are used, compared to the periodogram with the same amount of data (512 data points for the Monte Carlo simulation study and 896 data points for the wave-buoy comparisons).

\subsection{Inversion procedure}

As the modified covariance method is promising, the inversion procedure is carried out on its resulting AR PSD estimate. Figures 6.13, 6.14, 6.15 and 6.16 show different directional spectra which consist of the energy spectrum, mean direction and directional spread. They are obtained from spectra determined using the modified covariance method and the periodogram (with full data set), using the data originating 
from the OSCR system. We use the dual radar analysis to solve directional and amplitude ambiguities (see Chapter 2 sections 2.3 and 2.4) and the Wyatt inversion method (Wyatt 1990b). The modified covariance method is applied on the second collection of 5-minute measurements since this time is closer to the wave-buoy measurements. Also, it uses the model order recommended by one wave-buoy comparison (the "sea-truth" consideration): $p=30$ (see section 6.2.1).

The energy spectrum $S_{e n}(f)$ shows how the wave energy is proportionally distributed with frequency. For example in figure 6.13 , at the frequency $f=0.1 \mathrm{~Hz}$ we have $S_{e n}(f)=1.3 \mathrm{~m}^{2} / \mathrm{Hz}$ for the buoy. This means that waves travelling with the period $T=\frac{1}{0.1}=10 s$, have the same energy contribution. Low frequency waves, which are usually generated by distant storms outside the region covered, are swell (Brown et al., 1989). High frequency waves are wind sea; they are created by the local wind. The mean direction informs us of the direction taken by the different wave groups. From this direction, the directional spread shows how waves can spread. On the top of the graphs is written the day and time of the measurements (radar and buoy), the location and the significant wave height and mean period respectively for the radar and the buoy. Concerning the radar, another parameter is added. It is the mean difference in $\mathrm{dB}$ between the inverted and measured spectrum and is a measure of the convergence used in the inversion procedure. This convergence criterion must be less than 2 (Wyatt 1997e).

In figure 6.13, the two kinds of waves are clearly seen. The first peak at the frequency $0.1 \mathrm{~Hz}$ represents the swell and the second one at $0.2 \mathrm{~Hz}$ characterizes the wind sea which is less important in this case. By looking at the mean direction, the swell is propagating from the North and the wind-sea from the East. Good similarities between the wave-buoy measurements and the two spectral techniques, the modified covariance method and the periodogram, are obtained. Concerning the wave energy spectrum, it is the periodogram which gives a better superposition. However, the different wave parameters (significant wave height and mean period) extracted from the modified covariance method are closer to the buoy's.

Figure 6.14 shows a similarity between the periodogram and the modified covariance 
method which reveal a strong peak at $0.15 \mathrm{~Hz}$. This is not the case for the wave-buoy measurements. Both swell and wind sea are present even if they are not very well delimited. This explains the differences occurring in the mean direction. For the wave parameters, both spectral techniques are comparable.

In figure 6.15, the wave energy spectrum obtained from the AR PSD estimate contains more energy than the one obtained from the periodogram. But both of them are similar. It is difficult to tell if the first peak at $0.15 \mathrm{~Hz}$ represents swell and wind sea. Another peak occurs above $0.2 \mathrm{~Hz}$. For this case, both wave groups have the same direction; they are travelling on-shore. Concerning the wave parameters, it is the periodogram which gives better results.

The last figure 6.16 concerns only the modified covariance method since the periodogram revealed split Bragg peaks (see figures 4.10 and 4.13). A peak below $0.1 \mathrm{~Hz}$ appears in the wave-buoy energy spectrum and not in the different radar energy spectra. This is due to the fact that the radar range starts only at $0.1 \mathrm{~Hz}$ in this case and so nothing below this frequency can be detected. As can be seen, swell is propagating from the north and wind sea is travelling from East. Also, the wave parameters are close to the waverider's.

In conclusion, we showed that the AR PSD estimates obtained by the modified covariance method can be inverted and give similar results to the periodogram. Also, we showed that the inversion procedure can be still carried out when the periodogram is not useable because of the problem of split peaks. To give firm conclusions, further work is needed. The inversion procedure must be applied to more AR PSD estimates. 


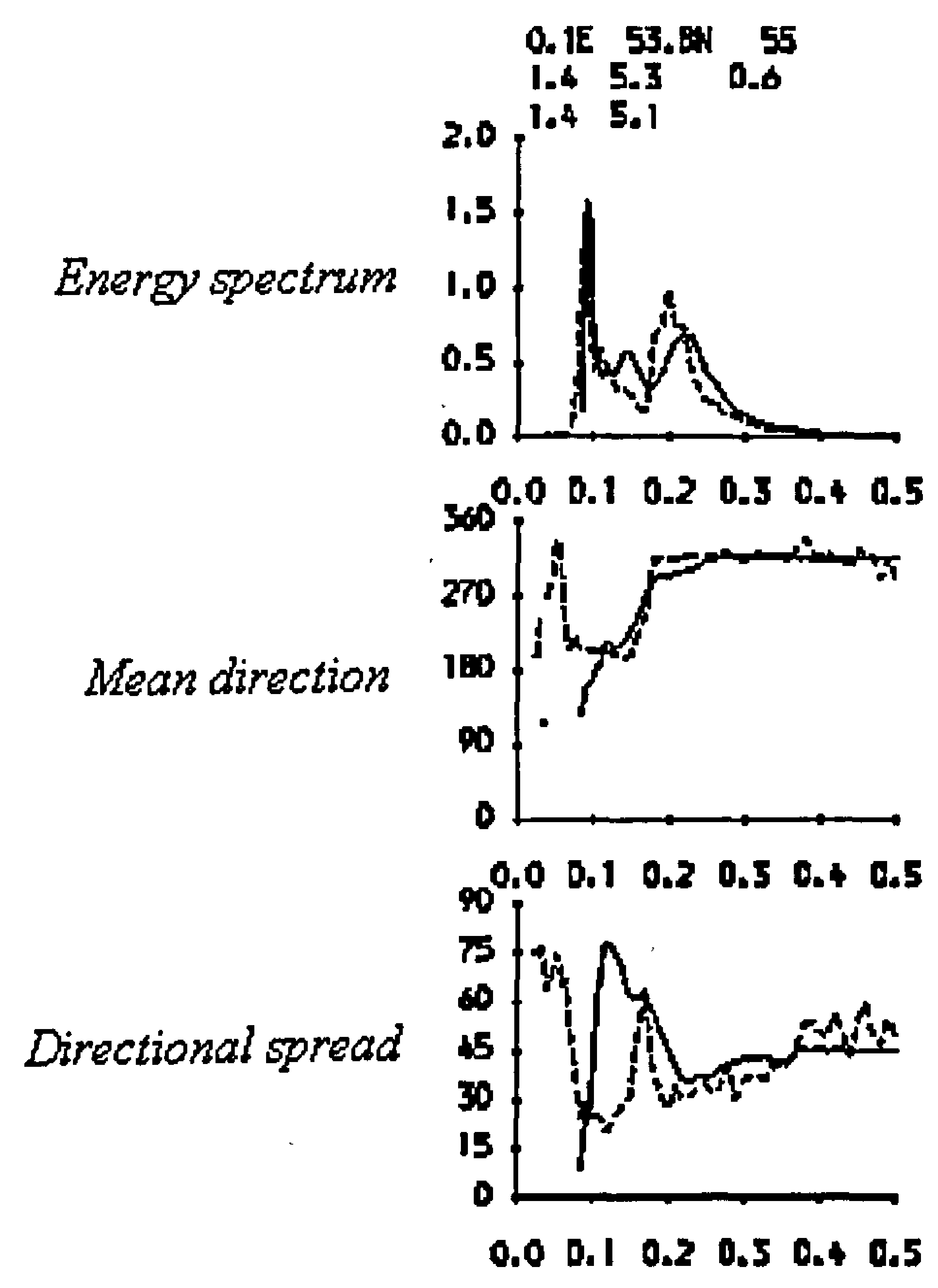

(a)

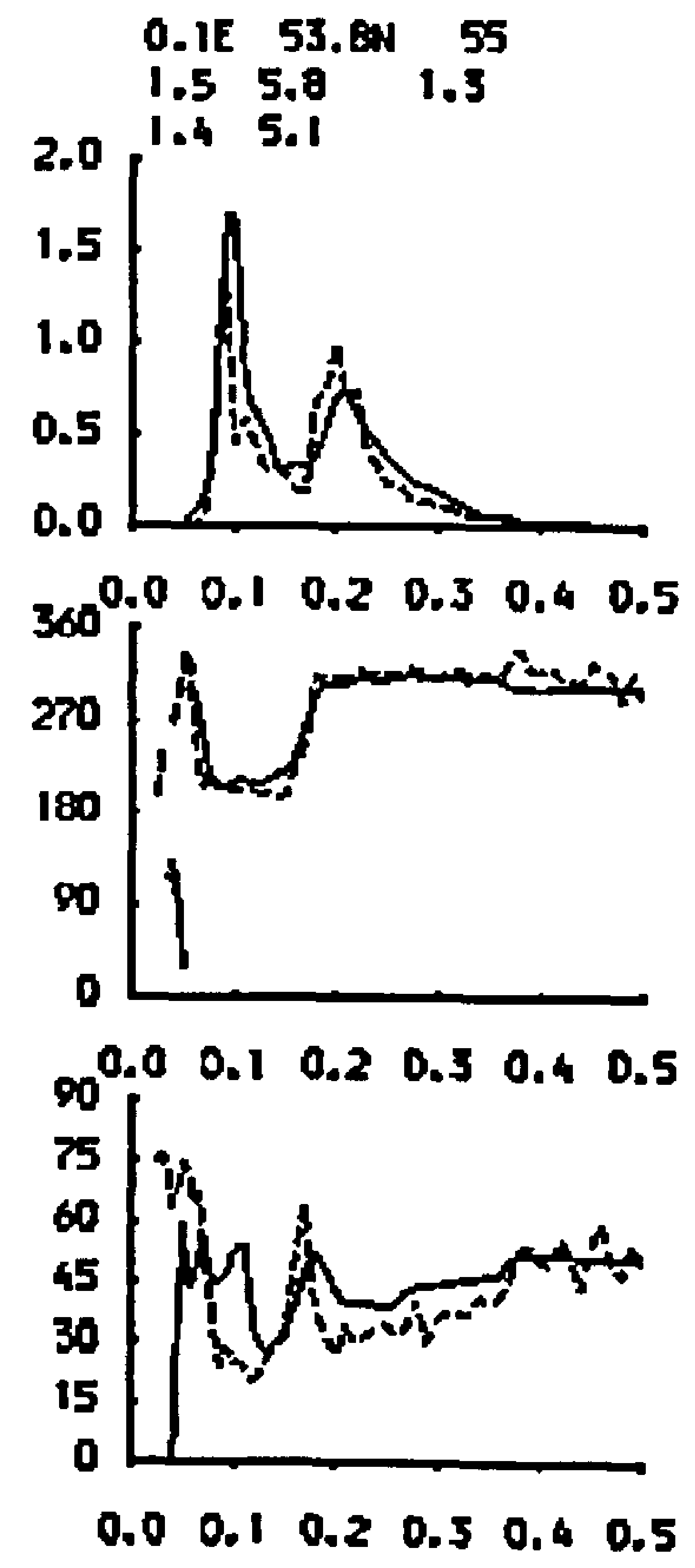

(b)

Figure 6.13: Full directional spectrum resulted from the modified covariance method (a) and the periodogram (b) and obtained from the cell 55 (measurement taken at $14 \mathrm{pm}$ on 21 December 1995), showing the energy spectrum, mean direction and spread as functions of frequency at locations measured from the North. Radar measurements are shown with a solid line and wave-buoy measurements with a dashed line. 


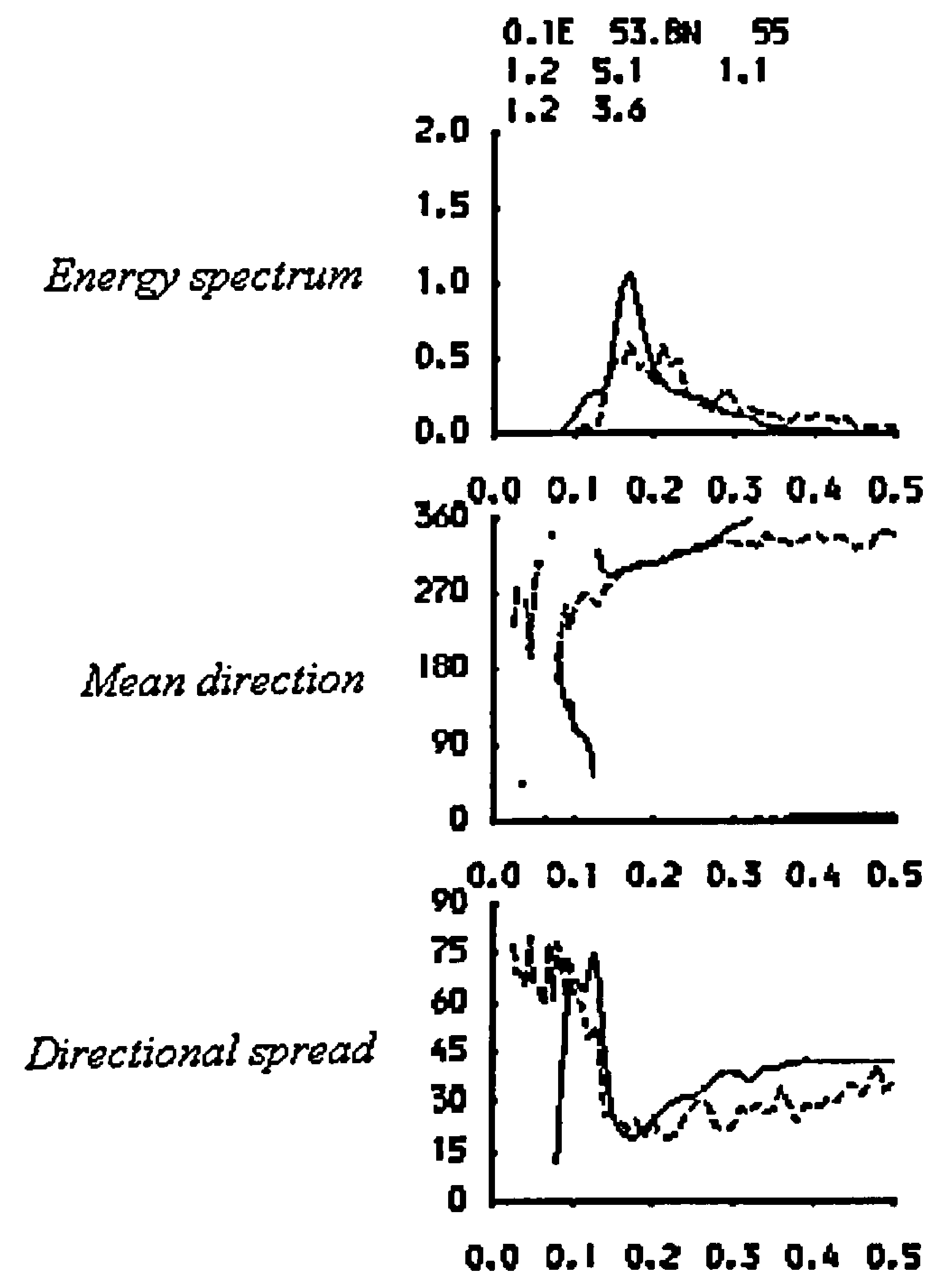

(a)

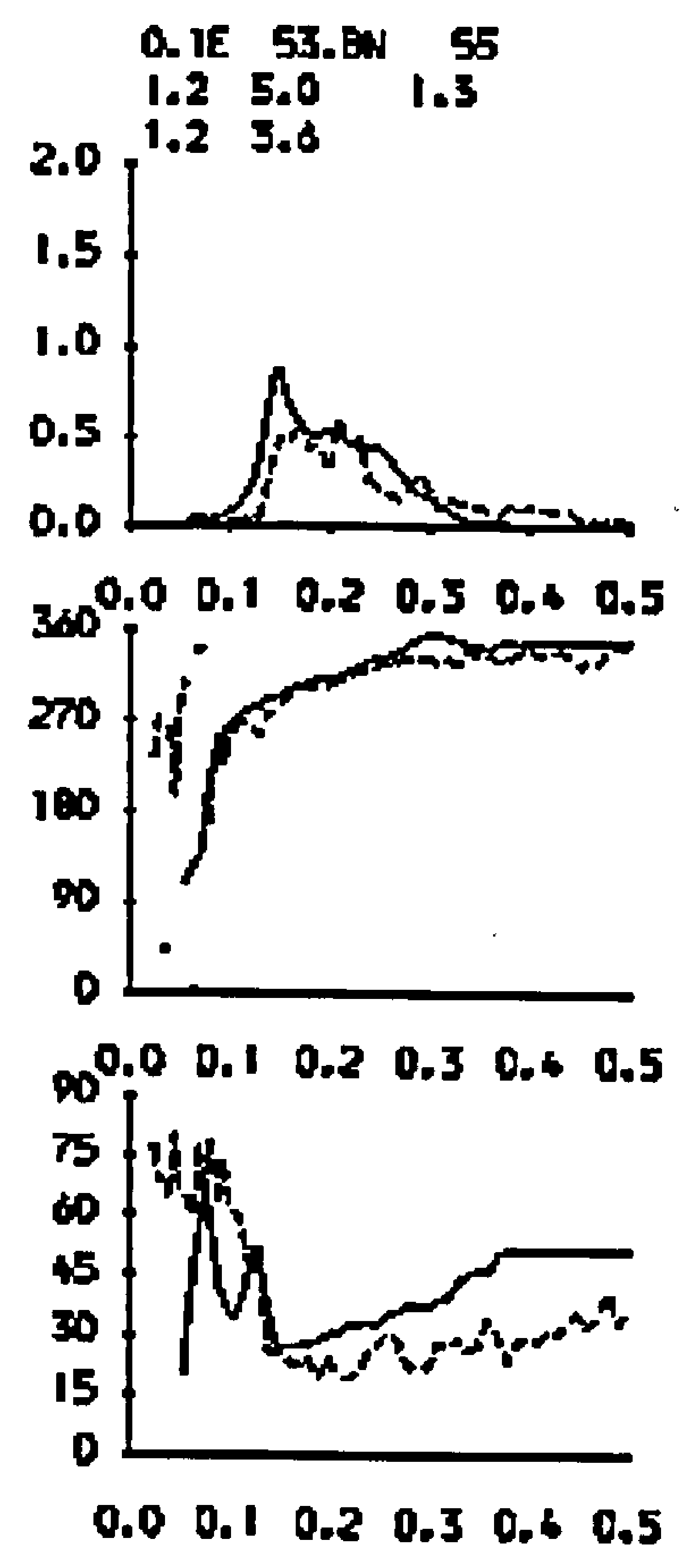

(b)

Figure 6.14: Full directional spectrum resulted from the modified covariance method (a) and the periodogram (b) and obtained from the cell 55 (measurement taken at 22pm on 9 January 1996), showing the energy spectrum, mean direction and spread as functions of frequency at locations measured from the North. Radar measurements are shown with a solid line and wave-buoy measurements with a dashed line. 


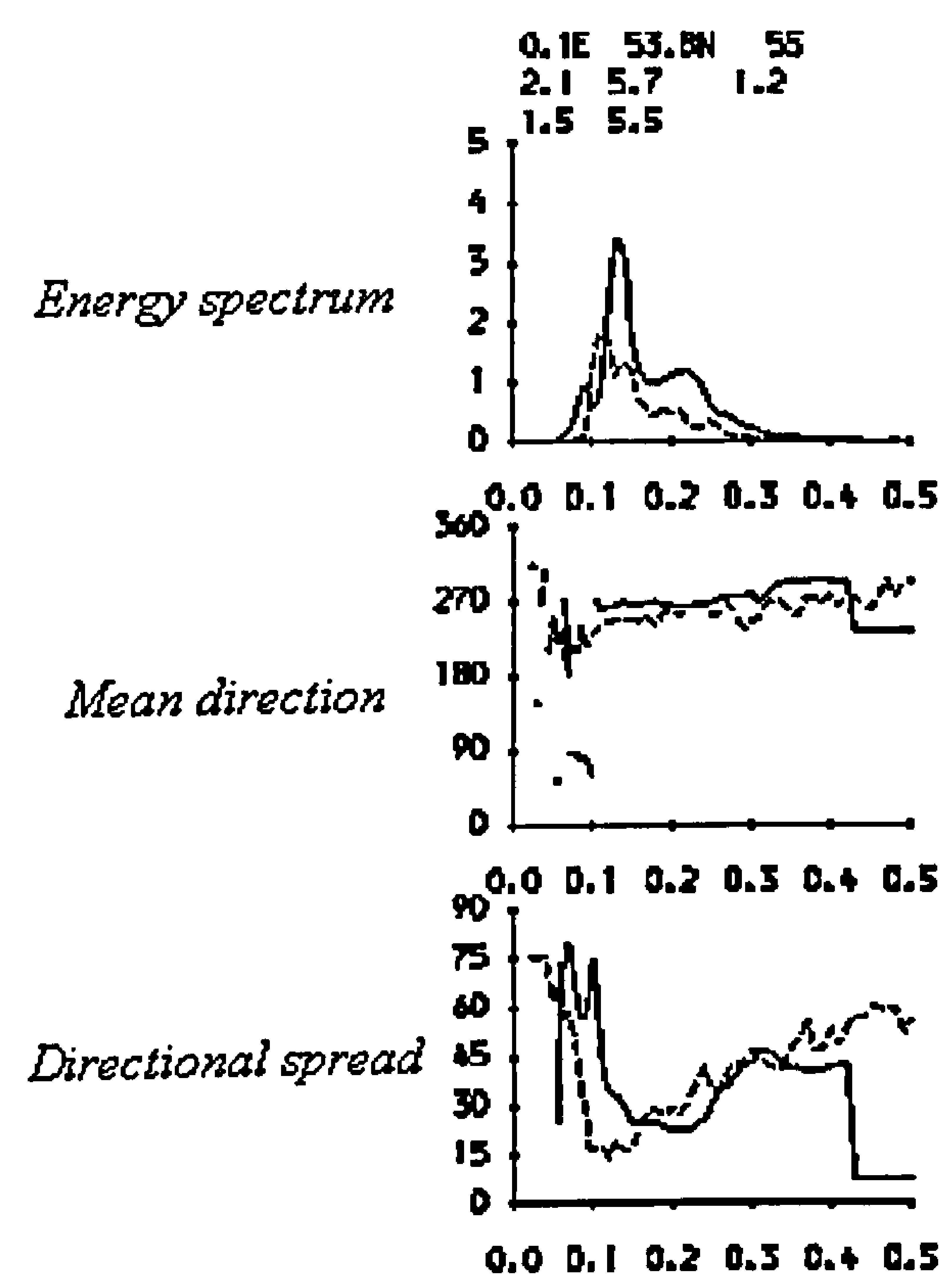

(a)

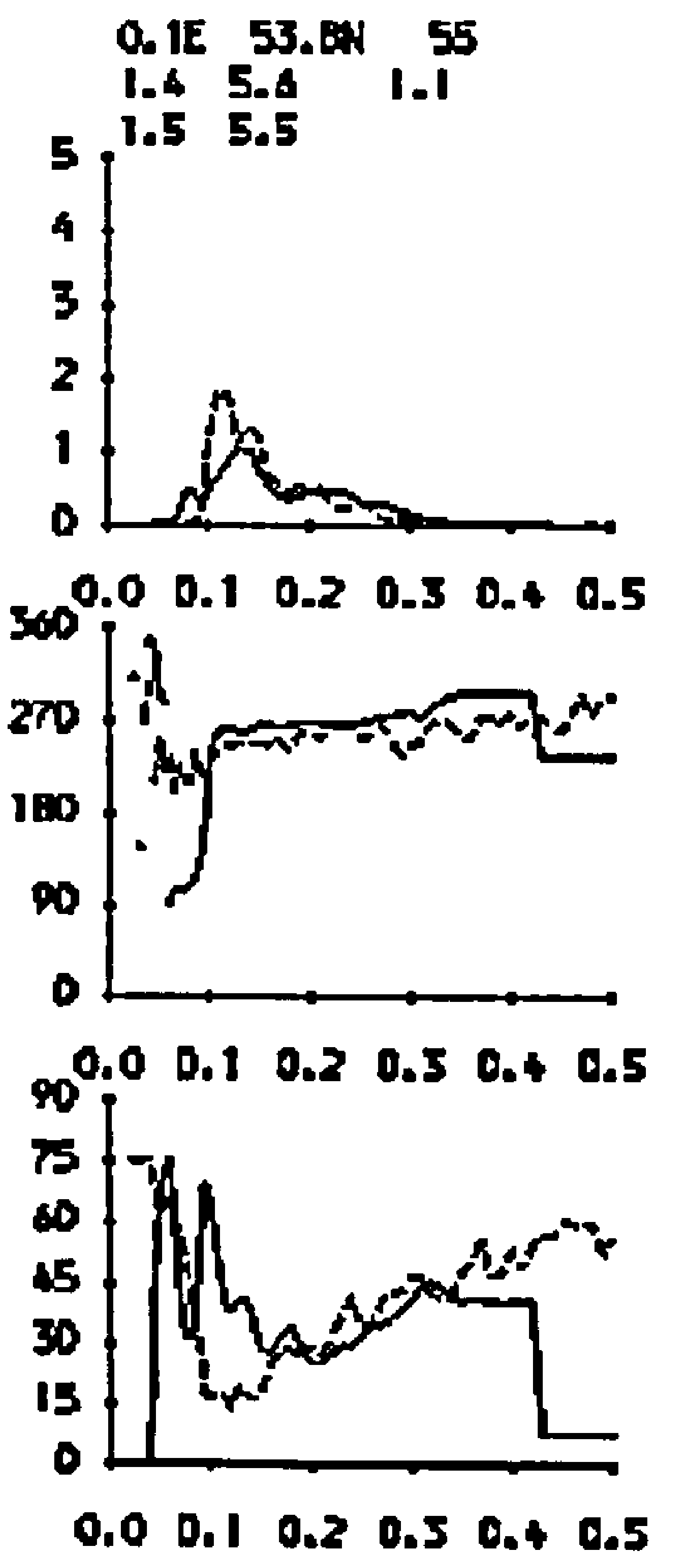

(b)

Figure 6.15: Full directional spectrum resulted from the modified covariance method (a) and the periodogram (b) and obtained from the cell 55 (measurement taken at 20pm on 1 January 1996), showing the energy spectrum, mean direction and spread as functions of frequency at locations measured from the North. Radar measurements are shown with a solid line and wave-buoy measurements with a dashed line. 


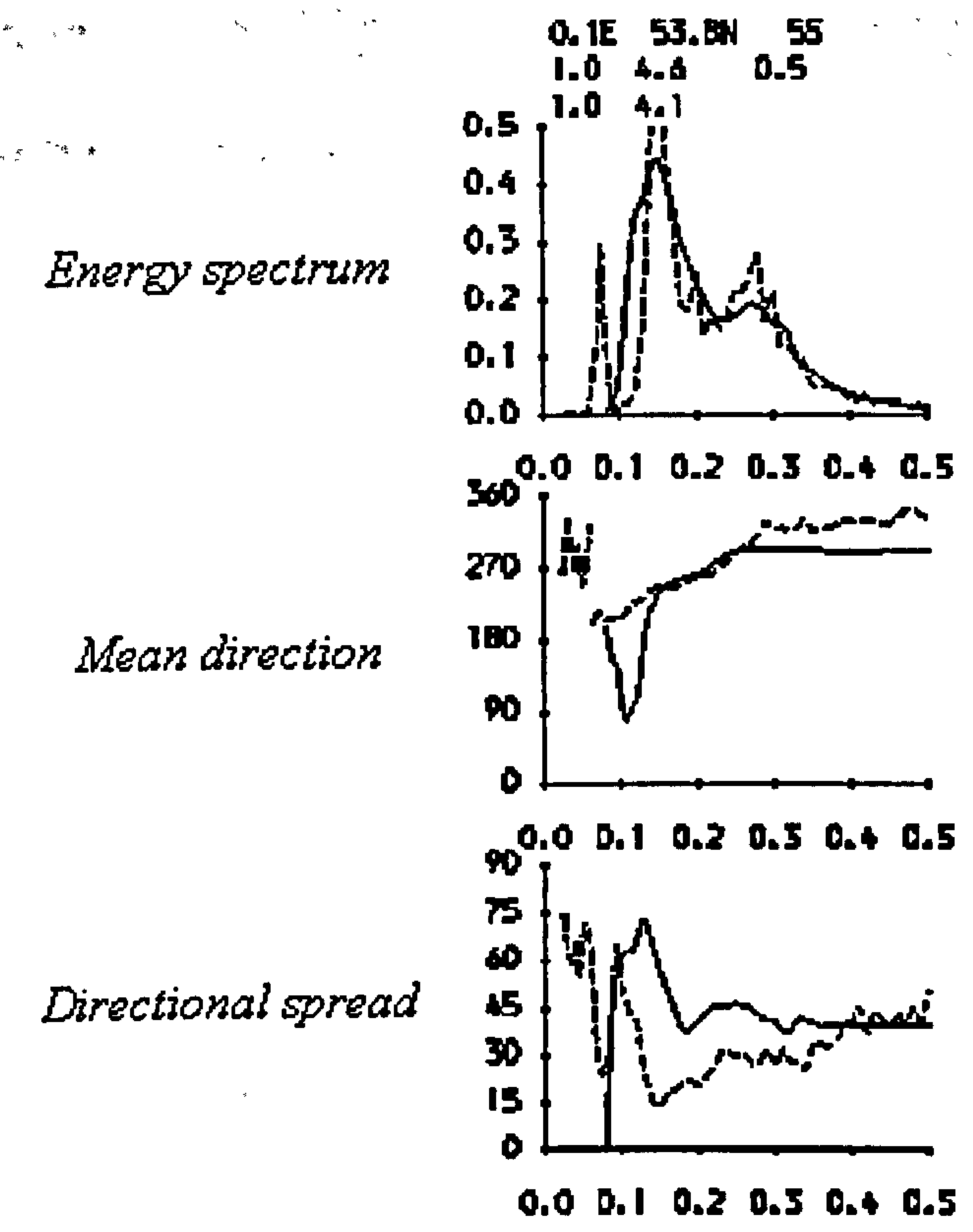

Figure 6.16: Full directional spectrum resulted from the modified covariance method obtained from the cell 55 (measurement taken at 12am on 3 January 1996), showing the energy spectrum, mean direction and spread as functions of frequency at locations measured from the North. Radar measurements are shown with a solid line and wavebuoy measurements with a dashed line. 


\section{Chapter 7}

\section{Evaluation of the Modified Covariance Method with the PISCES system}

For this HF radar system, this chapter contains only the results obtained by the two wave-buoy comparisons presented in Chapter 5 section 5.2. For the reasons given in Chapter 5 section 5.1.1, the Monte Carlo simulation study was not carried out with this system.

The objective is to compare the modified covariance method with the periodogram and give the accuracy of the wave parameters from extraction.

Since the radar measurements were taken continuously (or coherently) over half an hour, here we propose a data set length which produces the best first- and secondorder contributions. 


\subsection{Wave-buoy results}

\subsubsection{Wind direction, $\theta_{\omega}$}

Table 7.1 contains the mean difference and the standard deviation obtained for $\theta_{\omega}$ (see Chapter 5 section 5.2.2). As can be seen, the mean difference is slightly better for the periodogram. But, when $N \geq 1000$, it is the modified covariance method which is better in terms of the standard deviation. For the modified covariance method, less error is obtained when $p=30$ in general and this is reduced when $N=2000$. However, one can say that when $N \geq 1000$, the results are comparable.

In addition, the results obtained for $N=500$ confirm the comments presented in Chapter 4 section 4.4.2. It was shown that this data set length was not enough to get a good estimation of the difference in amplitude of the two Bragg peaks in other words a good estimation of the wind direction.

\subsection{2 "Sea-truth" consideration}

This wave-buoy comparison considers the wave-buoy measurements as "sea-truth" measurements (see Chapter 5 section 5.2.1).

Figures 7.1 and 7.2 show the mean and variance of the error (see Chapter 5 section 5.2), resulting from the difference between the wave-buoy measurements and the wave parameters extracted from the periodogram and the modified covariance method. Tables 7.2-7.5 show the different results obtained from the modified covariance method with four data set lengths and different model orders. In total, 88 PISCES files, each containing 4608 samples, are tested. Only good data are taken with the same criteria as used for the OSCR system presented in the previous chapter (see section 6.2).

Both radar and buoy systems work for around 30 minutes. The periodogram uses these 30-minute measurements; that is not the case for the modified covariance method, for which shorter measurements are applied. As we assume that these 30minute measurements are stationary in the sense that the sea-state is almost constant 
during this period of time, we can take the first $N$ data for the modified covariance method for the comparison.

Table 7.1

\begin{tabular}{|c|c|c|}
\hline \multirow{2}{*}{ Type } & \multicolumn{2}{|c|}{$\theta_{\omega}\left({ }^{\circ}\right)$} \\
\cline { 2 - 3 } & mean difference & standard deviation \\
\hline Periodogram $N=\mathbf{4 6 0 8}$ & 14 & 12.1 \\
\hline$N=\mathbf{5 0 0}$ AR(20) & 17.14 & 13.22 \\
\hline $\operatorname{AR}(30)$ & 16.82 & 13.14 \\
\hline $\operatorname{AR}(40)$ & 17.05 & 13.28 \\
\hline$N=\mathbf{1 0 0 0}$ AR(20) & 15.9 & 12.34 \\
\hline $\operatorname{AR}(30)$ & 15.83 & 11.64 \\
\hline $\operatorname{AR}(40)$ & 15.7 & 11.51 \\
\hline$N=1500$ AR(20) & 15.29 & 11.83 \\
\hline $\operatorname{AR}(30)$ & 14.97 & 10.71 \\
\hline $\operatorname{AR}(40)$ & 15.3 & 11.18 \\
\hline$N=2000$ AR(20) & 15.15 & 10.77 \\
\hline $\operatorname{AR}(30)$ & 14.36 & 10.35 \\
\hline $\operatorname{AR}(40)$ & 15.04 & 10.58 \\
\hline
\end{tabular}

Errors resulting from the comparison between the wave-buoy measurements and the wave parameter $\theta_{\omega}$ extracted from the periodogram with full data set and the modified covariance method with three different model orders for different data set length.

As our objective is to use shorter data sets with the modified covariance method, four different data set lengths are tested: $N=500,1000,1500$ and 2000. For the model order $p$, we use the same range as for the OSCR system: $20 \leq p \leq 40$. As can be seen in, tables 7.2, 7.3, 7.4 and 7.5 for the different data set lengths, less overall error is obtained when $p=30$, as for the OSCR system. 
Table 7.2

\begin{tabular}{|c|c|c|c|c|}
\hline \multirow{2}{*}{ Type } & \multicolumn{2}{|c|}{$H_{S}(\%)$} & \multicolumn{2}{c|}{$T_{\omega}(\%)$} \\
\cline { 2 - 5 } & Bias & Variance & Bias & Variance \\
\hline$p=20$ & 17.65 & 14.84 & 5.91 & 1.326 \\
\hline$p=30$ & 12.77 & 3.83 & 2.05 & 0.08 \\
\hline$p=40$ & 117.53 & 12921 & 295.38 & 10462 \\
\hline
\end{tabular}

Errors resulting from the "sea-truth" comparison between the wave-buoy measurements and the wave parameters ( $H_{S}$ and $T_{\omega}$ ) extracted from the modified covariance method with $N=500$.

Table 7.3

\begin{tabular}{|c|c|c|c|c|}
\hline \multirow{2}{*}{ Type } & \multicolumn{2}{|c|}{$H_{S}(\%)$} & \multicolumn{2}{c|}{$T_{\omega}(\%)$} \\
\cline { 2 - 5 } & Bias & Variance & Bias & Variance \\
\hline$p=20$ & 10.89 & 6.57 & 6.78 & 2.09 \\
\hline$p=30$ & 6.4 & 1.01 & 2.37 & 0.15 \\
\hline$p=40$ & 7.62 & 615.3 & 398.94 & 17920 \\
\hline
\end{tabular}

Errors resulting from the "sea-truth" comparison between the wave-buoy measurements and the wave parameters ( $H_{S}$ and $T_{\omega}$ ) extracted from the modified covariance method with $N=1000$.

Table 7.4

\begin{tabular}{|c|c|c|c|c|}
\hline \multirow{2}{*}{ Type } & \multicolumn{2}{|c|}{$H_{S}(\%)$} & \multicolumn{2}{c|}{$T_{\omega}(\%)$} \\
\cline { 2 - 5 } & Bias & Variance & Bias & Variance \\
\hline$p=20$ & 10.37 & 2.58 & 6.34 & 2.14 \\
\hline$p=30$ & 4.75 & 0.48 & 2.05 & 0.14 \\
\hline$p=40$ & 79 & 842.3 & 213.96 & 7298.7 \\
\hline
\end{tabular}

Errors resulting from the "sea-truth" comparison between the wave-buoy measurements and the wave parameters $\left(H_{S}\right.$ and $T_{\omega}$ ) extracted from the modified covariance method with $N=1500$. 
Table 7.5

\begin{tabular}{|c|c|c|c|c|}
\hline \multirow{2}{*}{ Type } & \multicolumn{2}{|c|}{$H_{S}(\%)$} & \multicolumn{2}{c|}{$T_{\omega}(\%)$} \\
\cline { 2 - 5 } & Bias & Variance & Bias & Variance \\
\hline$p=20$ & 10.74 & 3.31 & 6.99 & 2.34 \\
\hline$p=30$ & 4.64 & 6.28 & 1.91 & 0.09 \\
\hline$p=40$ & 71.76 & 811.02 & 144.41 & 3911.1 \\
\hline
\end{tabular}

Errors resulting from the "sea-truth" comparison between the wave-buoy measurements and the wave parameters $\left(H_{S}\right.$ and $\left.T_{\omega}\right)$ extracted from the modified covariance method with $N=2000$.

The next histograms (figures 7.1 and 7.2) show the results obtained from the periodogram with full data set, and the modified covariance method with four different data set lengths with the same model order $p=30$. As can be seen, the periodogram is generally better in terms of the given parameters. But, for certain values of $N$, the results obtained with the modified covariance method are comparable.

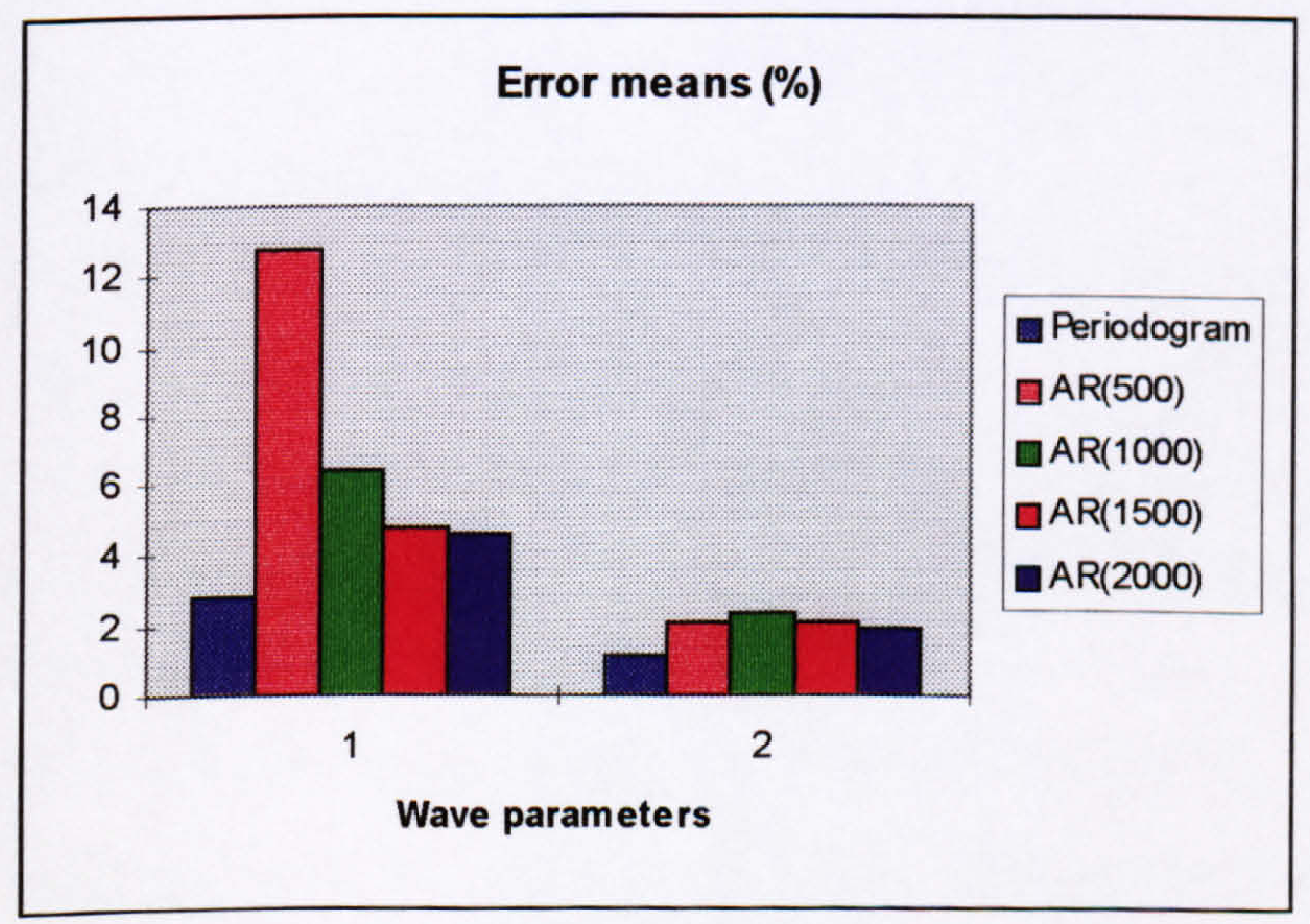

Figure 7.1: Histogram showing the mean of the error resulting from the comparison between the wave-buoy measurements and the wave parameters $\left(H_{S}\right.$ and $\left.T_{\omega}\right)$ extracted from the periodogram and modified covariance method. On the $x$-axis, the numbers 1 , and 2 represent $H_{S}$ and $T_{\omega}$ respectively. 


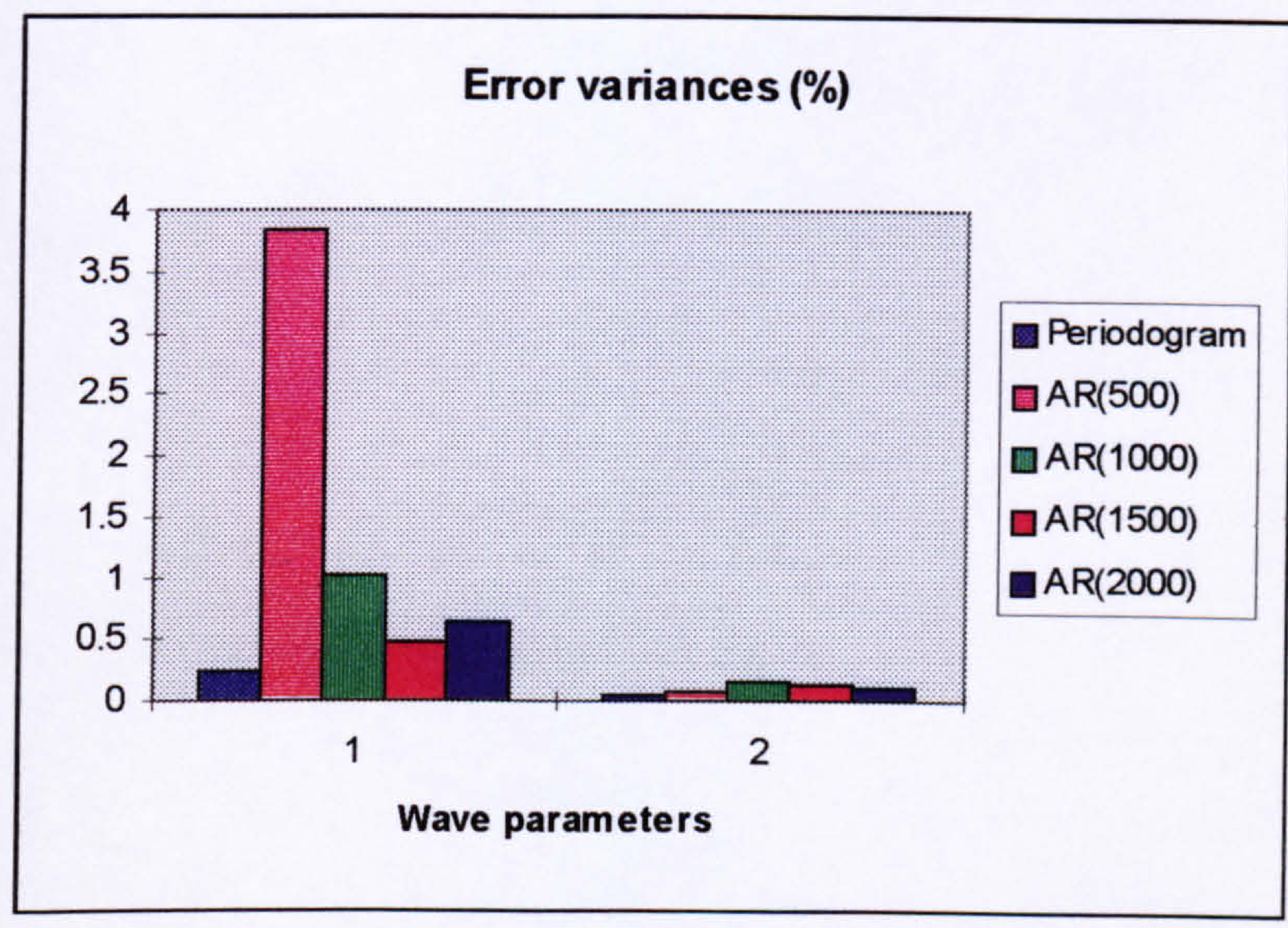

Figure 7.2: Histogram showing the variance of the error resulting from the comparison between the wave-buoy measurements and the wave parameters $\left(H_{S}\right.$ and $T_{\omega}$ ) extracted from the periodogram and modified covariance method. On the $x$-axis, the numbers 1 and 2 represent $H_{S}$ and $T_{\omega}$ respectively.

For the modified covariance method, when $N=500$, the global error in the significant wave height $H_{S}$ is very high, which confirms the statement seen in Chapter 4 section 4.4.2: $N=500$ is too low to get the second-order contribution. For this wave parameter, less error is obtained when $N=1500$.

As for the mean period $T_{\omega}$, the periodogram and the modified covariance method with the different values of $N$ provide comparable results.

In general, for $N \geq 1000$, comparable errors concerning $H_{S}$ and $T_{\omega}$ are produced by the modified covariance method. However, $N=1500$ is the recommended value to provide the most accurate wave parameters.

In general, the error mean is less than $5 \%$ for $H_{S}$ and $3 \%$ for $T_{\omega}$ and the error variance is less than $1 \%$ for $H_{S}$ and $0.2 \%$ for $T_{\omega}$. These results obtained for the error variance give a very good accuracy for the different wave parameters given that the waverider can produce $2.5-5 \%$ error (Allender et al. 1989) in the mean bias. 


\subsubsection{Symmetric regression}

This method assumes that errors can be produced in both measurements (see Chapter 5 section 5.2.2).

Table 7.6 contains the results obtained from the periodogram using full data set and the modified covariance method with four different data set lengths as for the previous comparison. As can be seen, the different errors are comparable for both techniques and are of the same magnitude as for the OSCR system (see Chapter 6 section 6.2.2). Also, these results confirm that $N=1500$ is the best data set length. A better accuracy for the wave parameters is provided by this number of data.

Concerning $H_{S}$, the periodogram and the modified covariance method are comparable. Either the bias or the variance is smaller in each spectral technique. For $T_{\omega}$, it is the periodogram which is better, particularly in terms of bias. The variance is equivalent for both techniques.

Table 7.6

\begin{tabular}{|c|c|c|c|c|}
\hline \multirow{2}{*}{ Type } & \multicolumn{2}{|c|}{$H_{S}(\%)$} & \multicolumn{2}{c|}{$T_{\omega}(\%)$} \\
\cline { 2 - 5 } & Bias & Variance & Bias & Variance \\
\hline Periodogram & -6.46 & 2.24 & 1.71 & 1.11 \\
\hline $\mathrm{AR}(N=500)$ & -15.16 & 9.98 & 7.64 & 1.39 \\
\hline $\mathrm{AR}(N=1000)$ & -6.1 & 6.05 & 8.48 & 1.57 \\
\hline $\mathrm{AR}(N=1500)$ & -4.61 & 4.54 & 8.32 & 1.34 \\
\hline $\mathrm{AR}(N=2000)$ & -5.33 & 4.38 & 8.48 & 1.15 \\
\hline
\end{tabular}

Errors resulting from the symmetric regression between the wave-buoy measurements and the wave parameters $\left(H_{S}\right.$ and $\left.T_{\omega}\right)$ extracted from the periodogram with full data set and the modified covariance method with four different data set lengths. 


\subsection{Conclusion}

The results of the wave-buoy comparisons show that the modified covariance method and the periodogram give similar results.

This leads us to determine an appropriate value for the data set length : $N=1500$ samples, for the first- and second-order parts of the spectrum. Therefore, this amount of data, which corresponds roughly to 10 minutes, is recommended for future HF radar experiments.

Figures 7.3 and 7.4 show the superpositions of the periodogram and the AR PSD estimate obtained from the PISCES files EB0319.011 and EB0328.008. The modified covariance method is applied with $N=1500$ and $p=30$. The two spectral estimates are also scaled to the integral under the first-order peak. This allows to compare the second-order part of the spectra which are directly related to the wave measurements. As can be seen, this superposition is very similar to the one shown in Chapter 4 figures 4.6 and 4.7 with $N=4608$.

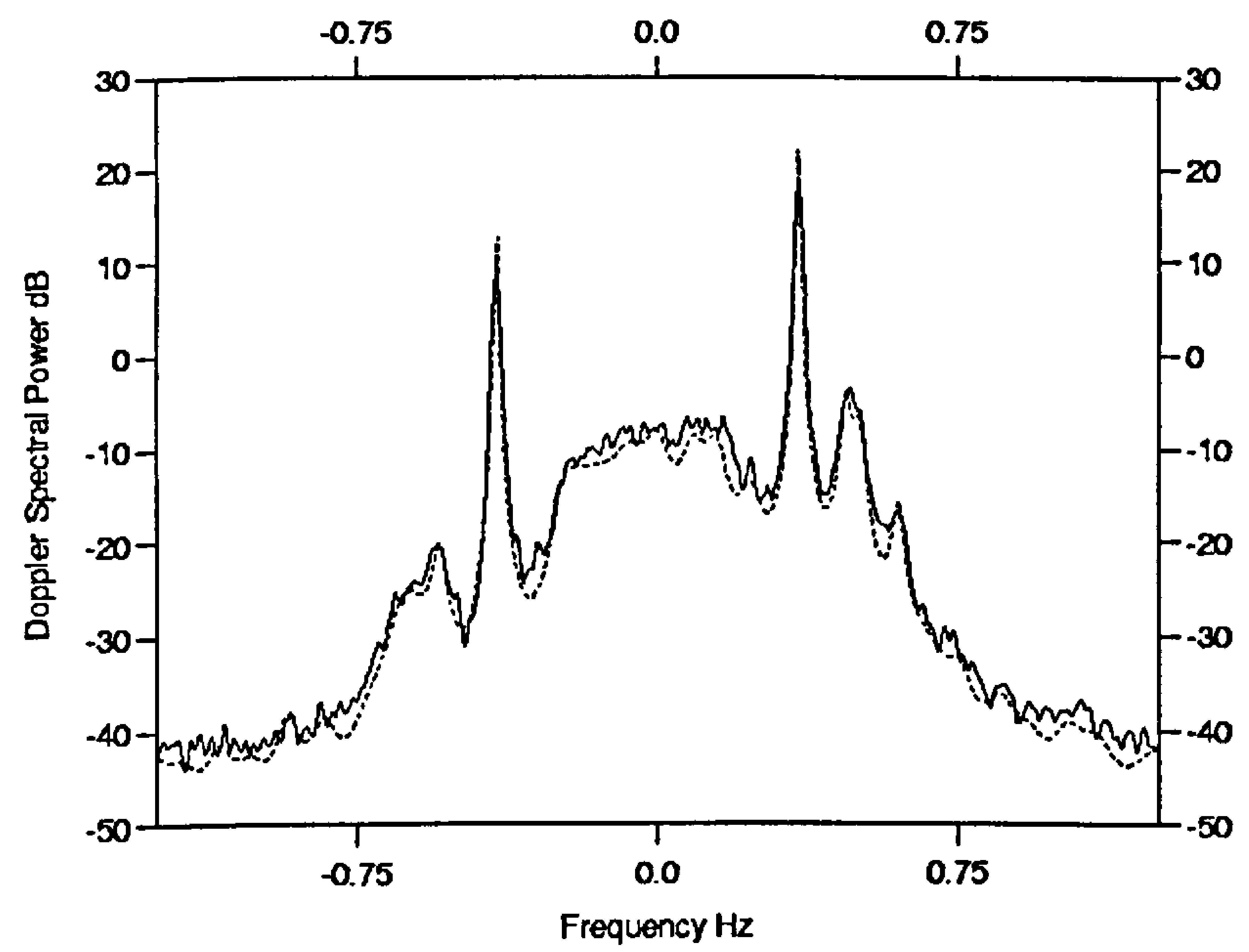

Figure 7.3: Superposition of the periodogram (solid line) and the AR PSD estimate (dotted line) with $N=1500$ and $p=30$, obtained from the PISCES file EB0319.011. 


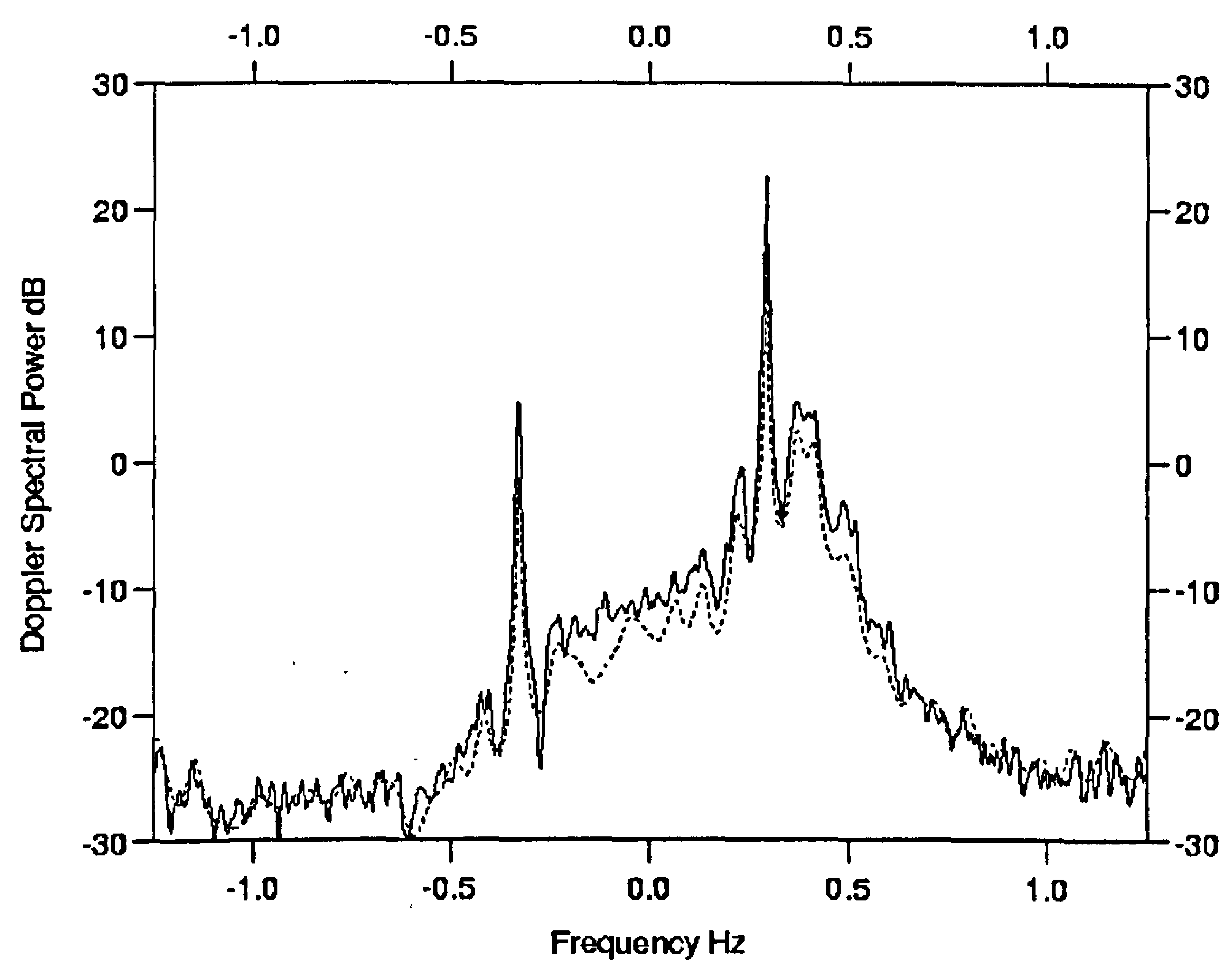

Figure 7.4: Superposition of the periodogram (solid line) and the AR PSD estimate (dotted line) with $N=1500$ and $p=30$, obtained from the PISCES file EB0328.008. 


\section{Chapter 8}

\section{Conclusions}

This thesis has been devoted to the investigation of modern spectral methods in order to monitor rapidly varying oceanographic conditions. The motivation has been twofold. Non-stationarity of the backscatter could occur and lead to the problem of split Bragg peaks in the Doppler spectrum. Even if this phenomenon of split Bragg peaks has not occured, the measurements may not have reflected correctly the sea conditions during this period of time. Finally, the modern spectral methods could provide stable spectral estimates using short data sets.

A certain quality of the Doppler spectrum is essential to get current and wave measurements. The choice of the modern spectral method was made on the first-order part of the spectrum, which is related to current measurements. The evaluation of this method was mainly done on the extraction of the wave parameters from the secondorder contribution.

\subsection{Modern spectral methods}

Four different spectral estimations were proposed in Chapter 3: 1. the autoregressive spectral estimation suitable for spectra with sharp peaks; 2. the autoregressive moving average spectral estimation able to represent different spectral shapes; 3 . the eigenanalysis-based frequency estimation producing peaks of narrowband spectra and 4. the recursive maximum likelihood estimation (RMLE) which is restricted to real data.

The recursive maximum likelihood estimation (RMLE) developed an estimate of the reflection coefficients and the order update of autoregressive parameters with the Levinson recursion. Since the requirement of the observed signal to be a real random 
process was too restrictive in the sense that the obtained Doppler spectrum was symmetric, this method was left aside.

The eigenanalysis-based frequency estimation was only worthwhile for the location of the two Bragg peaks, hence for surface current calculations. Since the second-order contribution was non-existent, we preferred not to work further with this technique.

The autoregressive moving average (ARMA) spectral estimation was also studied. This model could provide different spectral shapes. Good estimations of the Bragg peaks were obtained but only with small values of the model orders. Its computation time was also important. Therefore, we chose to work with the autoregressive (AR) spectral estimation.

This AR estimation showed the best results concerning the first-order part of the spectrum with no limitation on the model order, nature of the data and computation. Two methods were used to compute the AR power spectral density (PSD) estimate: the Burg method, which estimates the reflection coefficients subject to the Levinson recursion, and the modified covariance method based on a least-squared linear prediction approach. The choice between these efficient techniques was made on the fact that a phenomenon of split peaks, difficult to explain, could occur with the Burg method. (This phenomenon had nothing to do with the problem caused by nonstationarity of the data as mentioned above).

Therefore, we chose to work with the modified covariance method because this technique revealed the most efficiency and reliability concerning the first-order contribution.

\subsection{Evaluation of the modified covariance method}

\subsubsection{Qualitative assessment}

First, let us recall the mechanisms of the two HF radar systems.

The OSCR system collected data just for five minutes over a twenty-minute cycle; this corresponded to 896 samples. The classical spectral technique, the periodogram, used 
three separate five-minute data sets in order to be stable. Our interest was to apply the modified covariance method on one five-minute data set.

The PISCES system collected data continuously for about 30 minutes. This full data set of 4608 data points was used by the periodogram. In this case, we did not have any experimental constraints on the data set length for the application of the modified covariance method.

For both systems, Chapter 4 showed several superpositions of the periodogram and the AR PSD estimate resulted from the modified covariance method. Good similarities were obtained.

\subsubsection{Quantitative assessment}

Two kinds of comparison were proposed and described in Chapter 5:

- The Monte Carlo simulation study : it consisted of synthesizing signals whose spectral density function have similar characteristics to the observed signal. Since the true PSD function was known, it was straightforward to get the bias and variance, describing the quality of the spectral estimates. These error parameters were obtained in two different ways: directly on the spectral estimates, by comparing them with the "true" spectrum; and on the ocean wave parameters (wind direction, significant wave height and mean period), by extracting them from the different spectral estimates and the "true" spectrum.

This study was only applied to the OSCR system because disk space and computation time were too demanding for the PISCES system.

In Chapter 6, the results on the spectral estimates showed that the periodogram with full data set was, as expected, better since the periodogram is known to be the best method when using long data series. However, the results of the wave parameters obtained by the modified covariance method were comparable with the ones obtained by the periodogram. But when using short data sets, the modified covariance method is largely better than the periodogram. This was one of the objectives of this thesis. 
- The wave-buoy comparisons : By extracting the wave parameters from the different spectral estimates, we could compare these quantities with the wave-buoy measurements. Two methods were proposed. One considered the wave buoy measurements as "sea truth" measurements. The second one was a symmetric regression which took into account errors coming from both systems (radar and wave buoy systems). These comparisons were applied to both HF radar systems (Chapters 6 and 7).

With the OSCR system, the results obtained from the sea-truth comparison showed that the modified covariance method was better than the periodogram for the mean period and the wind direction. The symmetric regression showed that the periodogram and the modified covariance method had equal but different advantages. Also, these comparisons confirmed the mediocrity of the periodogram when using short data sets as pointed out with the Monte Carlo simulation study.

For the PISCES system, the different comparisons showed as well that these two spectral techniques were comparable.

This proves that the modified covariance method, with short data sets, and the periodogram, with full data sets, are equivalent in producing the Doppler spectrum. Therefore, these evaluations showed the quality of the modified covariance method using short data sets.

\subsection{Model order and data set length selections}

This work found a general model order, $p$, for the modified covariance method which is based on an autoregressive model. With the OSCR system in Chapter 6, a range was determined from the Monte Carlo simulation study: $20 \leq p \leq 40$. From this range, we found that the $p$-value of 30 gave the smallest error with the wave-buoy comparison.

Also, in Chapter 7 with reference to the PISCES system, this $p$-value was revealed to be the most suitable for different data set lengths $(N=500,1000,1500$ and 2000). 
Since the PISCES system was not limited by the data collection, we could look for a data set length to get the best Doppler spectrum.

In Chapter 4, we showed with this system that 500 samples were not enough to get a good estimation of the difference in amplitude of the two Bragg peaks, providing the wind direction. Chapter 7 confirmed this statement.

This chapter also recommended a data set length of 1500 samples (corresponding to a ten-minute collection) in order to get the most accurate wave parameters.

\subsection{Split peaks}

The first problem posed in the Introduction was the presence of split Bragg peaks in the Doppler spectrum. This implies the impossibility of proceeding with the inversion process which provides the full directional spectrum. In Chapter 4, this problem was shown to be the result of current variability and, by decreasing the time measurement, the modified covariance method reduced this problem since this method works well with short data sets. This showed the importance of using the modified covariance method for rapidly varying current conditions.

\subsection{Concluding remarks}

The reliability of the modified covariance method has been demonstrated. Good accuracy of the wave parameters was obtained.

By using short data sets, this method reduced the problem of split peaks and gives rise to thinner Bragg peaks.

Therefore, this method is efficient in the presence of rapidly varying oceanographic conditions.

In Chapter 6, an overall view of the inversion procedure was given with the AR spectral estimate obtained from this method. This also showed promising results, 
particularly when the periodogram cannot be used because of the problem of split peaks.

Finally, a data set length and a model order were recommended for the application of the modified covariance method: $N=1500$ and $p=30$. The amount of data is then reduced by a factor of four.

\subsection{Future work}

The stationarity of the radar data was pointed out in Chapter 2, through a wavelet analysis, and Chapter 5, with the Monte Carlo simulation study. One question arose: is the time measurement short enough to consider HF radar data to be stationary ? In other words, how long must the data sets be to give a stationary process ?

In order to answer this question, we started a wavelet analysis on some HF radar data. This time-frequency analysis with its scalogram showed how the energy was distributed in the time-frequency plane. We showed how interference could appear in the scalogram and when the signal was of better quality, in other words not drowned by some noise or interference. This pointed out the problem of split peaks by showing the main Bragg frequency moving during time. This analysis emphasized the use of the modified covariance method which by using short data sets reduced all these problems.

To generalize, further work is needed. We need to investigate more HF radar signals. Also, for this work, we used a particular wavelet basis and we do not know if another wavelet might or might not be more appropriate to our case (Torrence and Compo, 1998).

Also, to confirm the promising results obtained with the inversion procedure carried out on the modified covariance spectra, this inversion needs to be applied to a wide range of modified covariance spectra. 


\section{Bibliography}

Allender J, Audson T, Barstow S.F, Bjerken S, Krogstad H.E, Steinbakke P, Vartdal L, Borgman L.E and Graham C, 1989, "The Wadic Project: a Comprehensive Filed Evaluation of Directional Wave Instrumentation", Ocean. Engng., 16, No. 5/6, pp 505-536.

Barlett M.S, 1948, "Smoothing Periodograms from Time Series with Continuous Spectra", Nature, 161, pp 686-687.

Barrick D.E, 1977,' "Extraction of Wave Parameters from Measured HF Radar SeaEcho Doppler Spectra”, Radio Science, 3, No. 3., pp 415-424.

Brown J, Colling A, Park D, Phillips J, Rothery D and Wright J, 1989, “Waves, Tides and Shallow-Water Processes", Open University / Pergamon Press, London.

Chui C.K, 1992, “An Introduction to Wavelets, Wavelet Analysis and its Applications Series 1", Academic Press, Boston.

Cochran W.G and G.W Snedecor, 1989, "Statistical Methods, Eight Edition", Iowa State University Press.

Cohen L, 1989, "Time-Frequency Distributions _ A Review", Proc. IEEE, 77, No. 7, pp.

Crombie D.D, 1981, "Doppler Spectrum of Sea Echo at 13.56 Mc/S”, Nature, 175, pp 681-682.

Forget P, Broche P, De Maistre J.C, and Fontanel A, 1981, "Sea-State Frequency Features Observed by Ground Wave HF Doppler Radar", Radio Science, 16, No. 5, pp 917-925.

Harris F.J, 1978, "On the Use of Windows for Harmonic Analysis with the Discrete Fourier Transform", Proc. IEEE, 66, No. 1, pp 51-83.

Hasselmann K, 1971, "Determination of Ocean Wave Spectra from Doppler Radio Return from the Sea Surface”, Nature Phys. Sci., 229, pp 16-17.

Heron M.L, Dexter P.E and McGann B.T, 1985, "Parameters of the Air-Sea Interface by High-Frequency Ground-Wave Doppler Radar", Aust. J. Mar. Freshw. Res., 36, pp 655-670.

Herring R.W, 1980, "The Cause of Line Splitting in Burg Maximum-Entropy Spectral Analysis", IEEE Trans.on Acoustics, Speech and Signal Processing, A28, No. 6, pp 693-701.

Hickey K, Khan R.H and Walsh J, 1995, "Parametric Estimation of Ocean Surface Currents with HF Radar", IEEE Journal of Oceanic Engineering, 20, No. 2, pp 139-144. 
Holden G.J and Wyatt L.R, 1992, "Extraction of Sea State in Shallow Water Using HF Radar", IEE Proceedings- $F$, 139, No. 2, pp 175-181.

Howell R, 1990, "An Algorithm for the Extraction of Ocean Wave Spectra from Narrow Beam HF Radar Backscatter", M.Eng Thesis, Memorial Univ. of Newfoundland, Canada.

Kay S.M, 1988, “Modern Spectral Estimation”, Prentice-Hall.

Kay S.M, 1983, "Recursive Maximum Likelihood Estimation of AR processes", IEEE Trans. on Acoustics, Speech, and Signal Proc, ASSP-31, No. 1, pp 56-65.

Khan R.H, 1991, "Ocean-Clutter Model for High-Frequency Radar", IEEE Journal of Oceanographic Engineering, 16, No. 2, pp 181-188.

Kingsley S.P, 1986, "The coherence of HF Radar Sea Echoes", GEC Journal of Research, 4, No. 3, pp 203-210.

Kingsley S.P and Quegan S., 1992, “Understanding Radar Systems", McGraw-Hill International.

Kolmogoroff A.N, 1939, "Sur l'interpolation et l'extrapolation des suites stationnaires", C. R. Acad. Sci., 208, pp 2043.

Lipa B.J and Barrick D.E, 1986, "Extraction of Sea State by Radar, Remote Sensing of the Troposphere", NOAA/Environmental Resarch Laboratories, Boulder, Colorado, 21, No. 1, pp 81-100.

Lang S.W and McClellan J.H, 1980, "Frequency Estimation with Maximum Entropy Spectral Estimators", IEEE Transactions on Acoustics, Speech and Signal Processing, ASSP-28, No. 6, pp 716-724.

Mardia K.V, Jr., 1972, "Statistics of Directional Data", Academic Press (London and New York).

Marple S.L, Jr., 1987, “Digital Spectral Analysis with applications”, Prentice Hall.

Marple S.L, Jr., 1980, “A New Autoregressive Spectrum Analysis Algorithm”, IEEE Transactions on Acoustics, Speech and Signal Processing, ASSP-28, No. 4, pp 441-454.

Martin R.J and Kearney M.J, 1997, “A Remote Sea Current Sensing Using HF Radar: An Autoregressive Approach", IEEE Journal of Oceanic Engineering, 22, No. 1, pp 151-155.

Meyer Y, 1990, “Ondelettes et Operateurs 1", Hermann, Paris.

Paduan J.D and H.C Graber, 1997, "Introduction to High-Frequency Radar: Reality and Myth", Oceanography Society, 10, No. 2, pp 36-39.

Prandle D, Ballard G, Banqszek A, Bell P, Flatt D, Hardcastle P, Harrison A, Humphey J, Holdaway G, Lane A, Player R, Williams J and Wolf J, 1996, "The Holderness Coastal Experiment '93-'96”, Pol. Internal Report, No. 44.

Press, Flannery, Teukolsky, and 'Vetterling, 1988, "Numerical Recipies in C", Cambridge.

Press, Flannery, Teukolsky, and Vetterling, 1992, "Numerical Recipies in Fortran: the Art of Scientific Computing", Cambridge. 
Priestley M.B, 1981, “Spectral Analysis and Time Series”, London: Academic Press. Proakis J.G and Manolakis D.G, 1992, "Digital Signal Processing: Principles, Algorithms, and Applications", Macmillan ( second edition).

Sachs L, 1984, “Applied Statistics, a handbook of techniques", Springer-Verlag, New York Berlin Heidelberg Tokyo.

Schuster A, 1898, "On the Investigation of Hidden Periodicities with Application to a Supposed 26-Day Period of Meteorological Phenomena", Terr. Mag., 3, No. 1, pp 13-41.

Torrence C and Compo G, 1998, "A Pratical Guide to Wavelet Analysis", Bulletin of the American Meteorological Society, 79, pp 61-78.

Tucker M.J, 1991, “Waves in Ocean Engineering: Measurement, Analysis, Interpretation", Ellis Horwood in Marine Science.

Vaitkus P.J, Cobbold R.S.C and Johnston K.W, 1988, "A Comparative Study and Assessment of Doppler Ultrasound Spectral Estimation Techniques Part II : Methods and Results", Ultrasound in Med. and Biol., 14, No. 8, pp 673-688.

Wiener N, 1930, “Generalized Harmonic Analysis”, Acta. Math., 55, pp 117-258.

Wold H.O.A, 1938, "A Study in the Analysis of Stationary Time Series", Dissertation, reprinted by Almqvist and Wiksell Forlag in 1954.

Wyatt L.R, 1986, "The Measurement of the Ocean Wave Directional Spectrum from HF Radar Doppler Spectra", Radio Science, 21, pp 473-485.

Wyatt L.R, 1987, “Ocean Wave Parameter Measurements Using a Dual-Radar System: A Simulation Study”, Internal Journal Remote Sensing, 8, pp 881-891.

Wyatt L.R, 1990a, "Progress in the Interpretation of HF Sea Echo: HF Radar as a Remote Sensing Tool", IEE Proceedings, 137, No. 2, pp 139-148.

Wyatt L.R, 1990b, “A Relaxation Method for Integral Inversion Applied to HF Radar Measurement of the Ocean Wave Directional Spectrum", Int. J. Remote Sensing, 11, No. 8, pp 1481-1494.

Wyatt L.R, 1991, "High-Frequency Radar Measurements of the Ocean WaveDirectional Spectrum", IEEE Journal of Oceanic Engineering, 16, No. 1, pp 163169.

Wyatt L.R, 1994, "Coastal Surface Current and Wave Measurement with High Frequency Radar: Limitations and Prospects", published in "Mixing and Transport in the Environment", Wiley.

Wyatt L.R, 1995, "The Effect of Fetch on the Directional Spectrum of Celtic Sea Storm Waves", Journal of Physical Oceanography, 25, No. 26, pp 1550-1559.

Wyatt L.R and Ledgard L.J, 1996, “OSCR Wave Measurements - Some Preliminary Results", IEEE Journal of Oceanic Engineering, 21, No. 1, pp 64-76.

Wyatt L.R, Ledgard L.J and Anderson C.W, 1997a, "Maximum Likelihood Estimation of the Directional Distribution of $0.53 \mathrm{~Hz}$ Ocean Waves", Journal of Atmospheric and Oceanic Technology, 14, No. 3, pp 591-603. 
Wyatt L.R and Atanga J.N, 1997b, "Comparison of Inversion Algorithms for HF Radar Wave Measurements", IEEE Journal of Oceanic Engineering in press.

Wyatt L.R and Ledgard L.J, 1997c, "Measurement of Ocean Waves Using the OSCR HF Radar System", University of Sheffield.

Wyatt L.R, 1997d, "SCAWVEX : Surface Current and Wave Variability Experiment", Scientific Report for the period 1/2/96-31/1/97.

Wyatt L.R, 1997e, "Non-Linear Inversion of HF Radar Backscatter", University of Sheffield.

Yule G.U, 1927, "On a Method of Investigating Periodicities in Disturbed Series", Philos. Trans. R. Soc. London, 226, pp 267-298. 


\section{Appendix A}

\section{Some corrected codes}

This section contains two parts of C-programs, in which there are errors in the published code: (1) the Kolmogorov-Smirnov test (see Chapter 2 section 2.5.1) and (2) the procedure TEST of the recursive MLE method (see Chapter 3 section 3.4).

\section{A.1 Kolmogorov-Smirnov test}

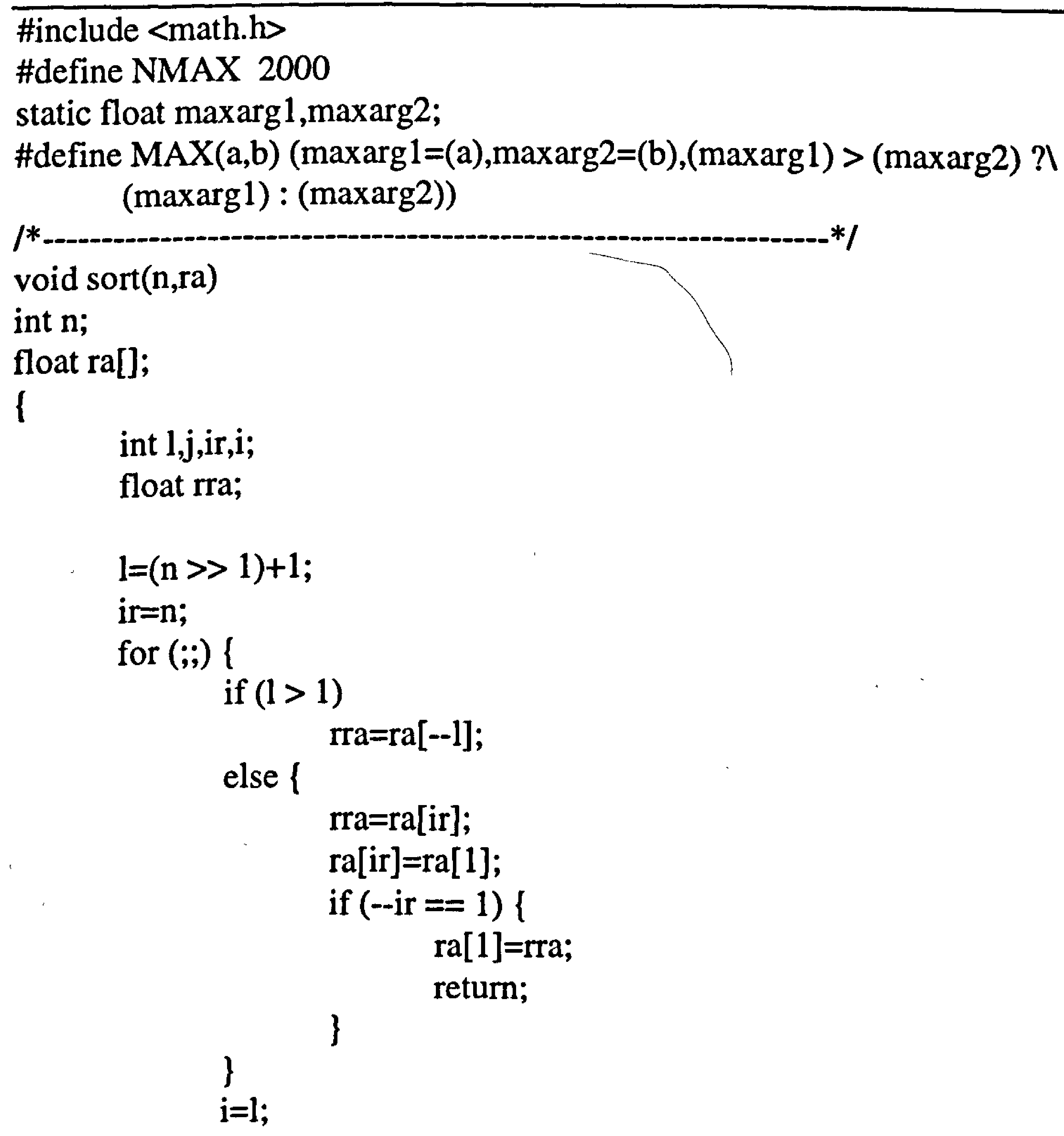




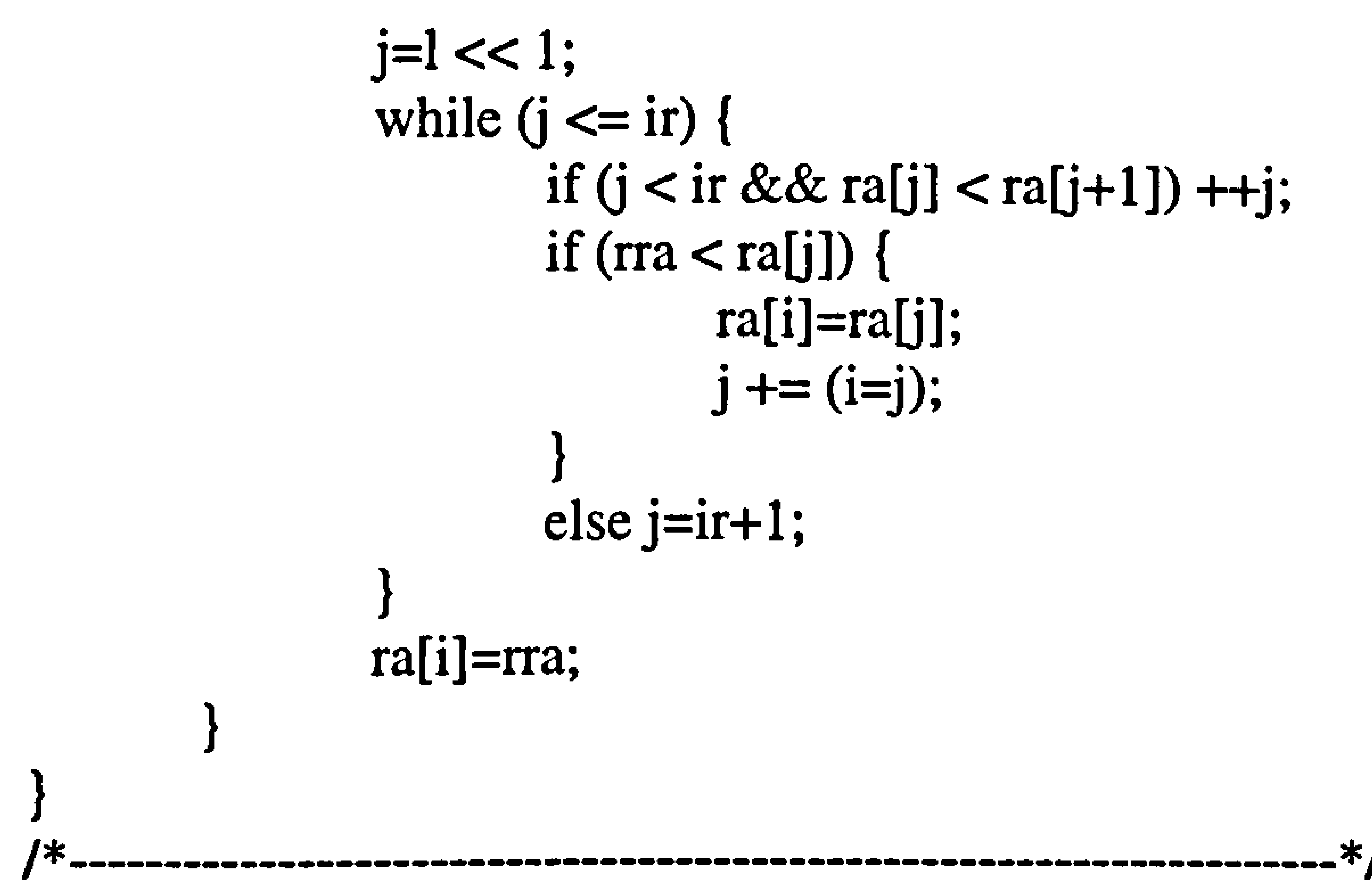

\#define EPS1 0.001

\#define EPS2 1.0e-8

float probks (alam)

float alam;

\{

int $\mathrm{j}$;

float $a 2$, fac $=2.0$, sum $=0.0$, term,termbf $=0.0$;

$\mathrm{a} 2=-2.0 *$ alam $*$ alam;

for $(j=1 ; j<=100 ; j++)\{$

term $=$ fac $* \exp \left(a 2 *_{j} *_{j}\right)$;

sum $+=$ term;

if $($ fabs (term) $<=$ EPS $1 *$ termbf $\|$ fabs(term) $<=$ EPS $2 *$ sum) return sum;

fac $=-f a c$;

termbf $=$ fabs(term);

\}

return 1.0;

\}

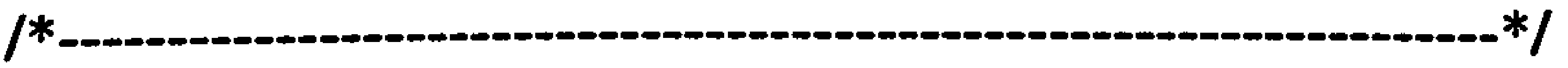

void ksone (data,n,func,d,prob,mean,var)

$/^{*}$ given an array data[] and given a cumulative distribution function

/* func ranging from 0 to 1 , this routine returns the K-S statistic */

$/ * \mathrm{~d}$ and the sigignificant level prob. Small values of prob show that $* /$

$/ *$ the cumulative distribution function of data is significantly */

$/ *$ different from func. The array data is modified by being sorted */

$/ *$ into ascending order.

$/ *$ mean and var are the mean and variance of the array data

float data[1],*d, ${ }^{\text {prob,mean,var; }}$

float (*func)();/* ANSI: float (*func)(float); */

int $\mathrm{n}$;

l

int $\mathrm{j}, \mathrm{i}, \mathrm{l}, \mathrm{nl}$;

float fn,ff,en,dt,aux;

float FO[NMAX],FN[NMAX]; 


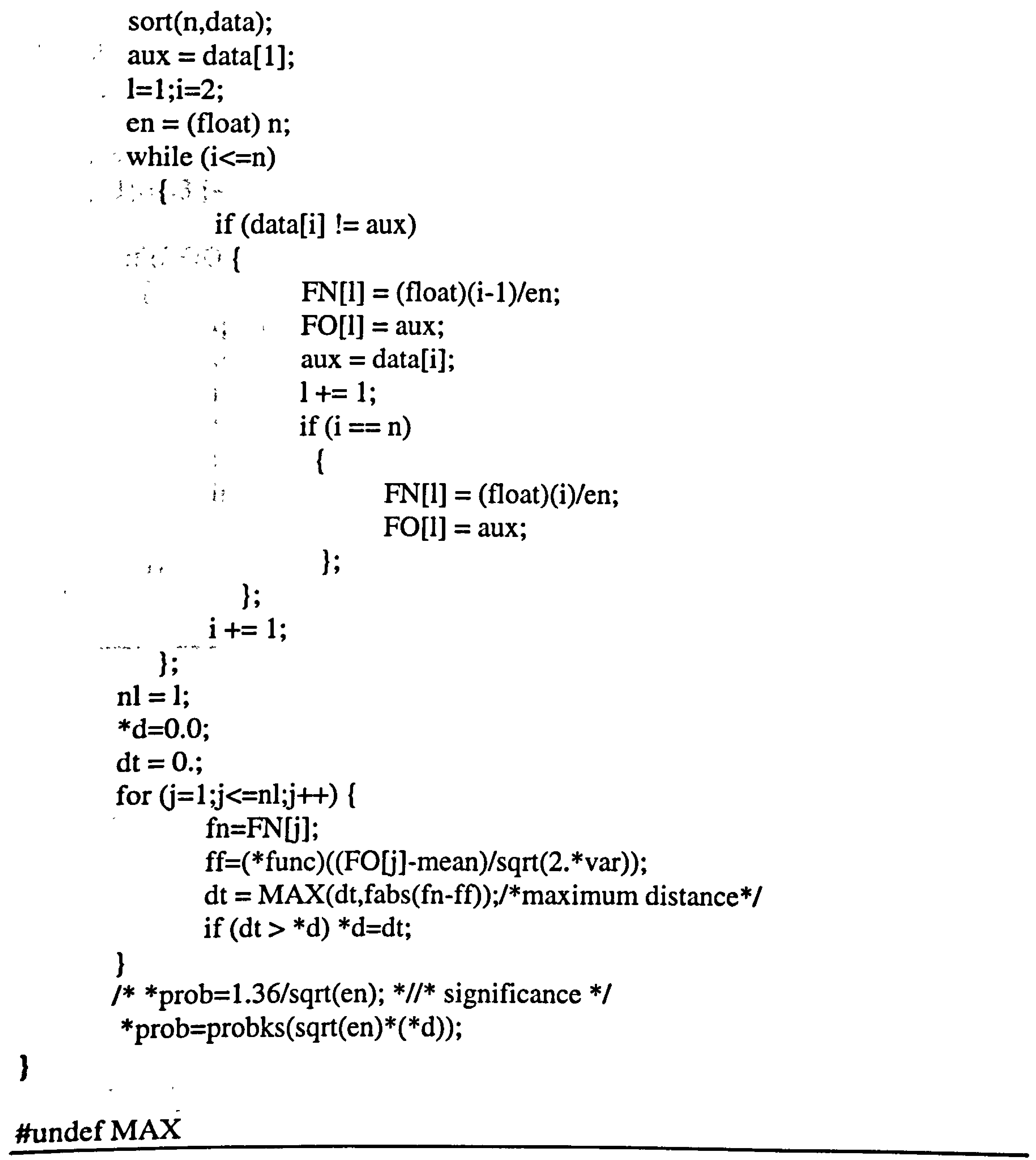

\section{A.2 Procedure TEST}

\section{void TEST (ROOT,C,D,E,I,N,XKEST)}

I* Check the 3 real roots of a cubic equation to determine which */

$I^{*}$ one is the maximum likelihood estimate of the reflection */

$1 *$ coefficient

double ROOT[3],C,D,E, ${ }^{*}$ XKEST; 
int $\mathrm{I}, \mathrm{N}$;

I

double fmax,q,f;

int $\mathbf{j}$;

$f \max =-10000000$;

for $(j=1 ; j<=3 ; j++)$

l

if $(\operatorname{ROOT}[\mathrm{j}-1]>=-1 . \&$ ROOT $[\mathrm{j}-1]<=1$.

\{

$\left.\mathrm{q}=\mathrm{fabs}\left(\mathrm{E}+2 .{ }^{*} \mathrm{ROOT}[\mathrm{j}-1]\right]^{*} \mathrm{C}+\mathrm{ROOT}[\mathrm{j}-1] * \mathrm{ROOT}[\mathrm{j}-1] * \mathrm{D}\right) / \mathrm{N}$;

$\mathrm{q}=\operatorname{pow}(\mathrm{q}, 0.5 * \mathrm{~N})$

$\mathrm{f}=\mathrm{fabs}(1 .-\mathrm{ROOT}[\mathrm{j}-1] *$ ROOT $[\mathrm{j}-1])$;

$\mathrm{f}=\operatorname{pow}\left(\mathrm{f}, 0.5^{*} \mathrm{I}\right)$;

$\mathrm{f}=\mathrm{f} / \mathrm{q}$; printf("f \% $\mathrm{fln}$ ",f);

if $(f>f \max )\{*$ XKEST $=$ ROOT $[j-1]$;

$\mathrm{fmax}=\mathrm{f} ;$;

\}

\};

I 


\section{Appendix B}

\section{Burg method}

This appendix describes the Burg method which was introduced in Chapter 3 section

3.2, used for autoregressive spectral estimation. This method is based on a orderrecursive least-squares approach. It minimizes the forward and backward linear prediction errors with the constraint that the autoregressive parameters satisfy the Levinson recursion:

$$
a_{p}(n)=a_{p-1}(n)+k_{p} a_{p-1}^{*}(p-n),
$$

where $k_{p}=a_{p}(p)$ is known as the reflection coefficient.

The forward and backward linear prediction errors are respectively :

$$
\left\{\begin{array}{c}
e_{p}^{f}(n)=x(n)+\sum_{k=1}^{p} a_{p}(k) x(n-k) \\
e_{p}^{f}(n)=x(n-p)+\sum_{k=1}^{p} a_{p}^{*}(k) x(n-p+k)
\end{array}\right.
$$

where $\{x(n)\}$ are the $N$-point data sequence $x(1), \ldots, x(N)$ which estimate the $p$ thorder AR parameters.

By substituting of equation (B.1) into these definitions, we get the following recursive relationships for the different linear prediction errors:

$$
\left\{\begin{array}{l}
e_{p}^{f}(n)=e_{p-1}^{f}(n)+k_{p} e_{p-1}^{b}(n-1) \\
e_{p}^{b}(n)=e_{p-1}^{b}(n-1)+k_{p}^{*} e_{p-1}^{f}(n)
\end{array}\right.
$$

At each order $p$, the arithmetic mean of the forward and backward linear prediction errors is:

$$
\rho_{p}^{f b}=\frac{1}{2 N} \sum_{n=p+1}^{N}\left\{\left|e_{p}^{f}(n)\right|^{2}+\left|e_{n}^{b}(n)\right|^{2}\right\}
$$


The objective is to minimize this error which is subject to the recursion given by equation (B.3). So : $\rho_{p}^{f b}$ is a function of the reflection coefficient $k_{p}$, since the prediction errors from order $p-1$ will be known.

This leads to set the complex derivative of equation (B.4) to zero:

$$
\frac{\partial \rho_{p}^{f b}}{\partial \Im m\left(k_{p}\right)}+i \frac{\partial \rho_{p}^{f b}}{\partial \Re e\left(k_{p}\right)}=0
$$

Thus, an estimate of the reflection coefficient is obtained:

$$
\hat{k}_{p}=\frac{-2 \sum_{n=p+1}^{N} e_{p-1}^{f}(n) e_{p-1}^{b^{*}}(n-1)}{\sum_{n=p+1}^{N}\left\{\left|e_{p-1}^{f}(n)\right|^{2}+\left|e_{p-1}^{b}(n-1)\right|^{2}\right\}}
$$

Once this reflection coefficient is estimated, the AR parameters can be calculated. Thus the AR PSD estimate $\underset{A R}{\hat{p}}(f)$ can be obtained:

$$
\underset{A R}{\hat{p}}(f)=\frac{T \hat{\rho}}{\left|1+\sum_{n=1}^{p} \hat{\mathrm{a}}_{\mathrm{p}}(n) e^{-2 j \pi f n T}\right|^{2}}
$$

where $T$ is the sample interval,

$\hat{\rho}$ is an estimate of the driving noise variance, and $\hat{a}_{p}(n)$ are the AR parameter estimates.

For a $p$ th-order model and $N$ data samples, this algorithm requires $3 N p-p^{2}$ complex additions and multiplications, as well as $p$ real divisions and element storage of $3 N+p$.

The advantages of the Burg method are:

- it results in high-frequency resolution.

- it yields a stable AR model since the normalization factors in the denominator of equation (B.6) ensures that the estimate of the reflection coefficient has magnitude less than 1.

- it is computationally efficient.

Its disadvantages are: 
- the spectrum of the signal may have a single sharp peak, but this method may result in two or more closely spaced peaks. This line splitting problem difficult to define was presented in Chapter 3 section 3.2. This was the reason we preferred to proceed with another autoregressive spectral method, the modified covariance method.

- for high-order models, it introduces spurious peaks.

- for sinusoidal signals in noise, it exhibits a frequency shift from the true frequency, resulting in a frequency bias (especially in short data sets). 


\section{Appendix C}

\section{Recursive Maximum Likelihood Estimation}

This section describes the Recursive Maximum Likelihood Estimation (RMLE) introduced in Chapter 3 section 3.5. This technique is an approximation to the true MLE. It operates in a recursive model order fashion, which allows to fit higher order models to the data. This method is restricted to real data.

This method develops an estimate of the reflection coefficients, $k_{p}$, and the order update of the autoregressive coefficients, $a_{p}(1), \ldots, a_{p}(p)$, which are provided by the Levinson recursion given in Appendix B equation (B.1).

Let consider a zero-mean Gaussian autoregressive process of order $p$ :

$$
\mathrm{X}=[x(1) \ldots x(N)]^{T} .
$$

Then, the probability density function (PDF) of $\mathbf{X}$ is

$$
p\left(\mathbf{X} \mid \mathbf{a}, \sigma^{2}\right)=\frac{1}{(2 \pi)^{N / 2}|\mathbf{R}|^{1 / 2}} \exp \left(-\frac{1}{2} \mathbf{X}^{T} \mathbf{R}^{-1} \mathbf{X}\right)
$$

where $\mathbf{a}=[a(1) \ldots a(p)]^{T}$ is the AR parameter vector, $\sigma^{2}$ is the white noise variance and $\mathbf{R}=E\left(\mathbf{X X}^{T}\right)$ is the covariance matrix ( $E$ is the expectation).

The true MLE of $\mathbf{a}, \sigma^{2}$ is found by maximizing $\ln p\left(\mathbf{X} \mid \mathbf{a}, \sigma^{2}\right)$ with respect to $\mathbf{a}$, $\sigma^{2}$

Since $\mathbf{X}$ is a zero-mean and autoregressive process, we can rewrite $\mathbf{R}$ as

$$
R(k)=E(X(t) X(t+k))=\sigma^{2} R_{f}(k),
$$


where $\mathbf{R}_{f}=Z^{-1}\left[\frac{1}{A(z) A\left(z^{-1}\right)}\right]$ is the filter autocorrelation function $\left(Z^{-1}\right.$ denotes the inverse $Z$ transform and $\left.A(z)=1+\sum_{k=1}^{p} a(k) z^{-k}\right)$.

Thus, the equation (C.1) becomes

$$
p\left(\mathbf{X} \mid \mathbf{a}, \sigma^{2}\right)=\frac{1}{(2 \pi)^{N / 2} \sigma^{N}\left|\mathbf{R}_{f}\right|^{1 / 2}} \exp \left(-\frac{1}{2 \sigma^{2}} \mathbf{X}^{T} \mathbf{R}_{f}^{-1} \mathbf{X}\right)
$$

Then, this probability is maximizing with respect to $\sigma^{2}$ because $\mathbf{R}_{f}$ depends only upon the filter parameters:

$$
\begin{aligned}
& \frac{\delta \ln p\left(\mathbf{X} \mid \mathbf{a}, \sigma^{2}\right)}{\delta \sigma^{2}}=-\frac{N}{2 \sigma^{2}}+\frac{1}{2 \sigma^{4}} \mathbf{X}^{T} \mathbf{R}_{f}^{-1} \mathbf{X}=0 \\
& \Rightarrow \sigma^{2}=\frac{1}{N} \mathbf{X}^{T} \mathbf{R}_{f}^{-1} \mathbf{X} .
\end{aligned}
$$

Substituting (C.5) into (C.3), we now need to maximize

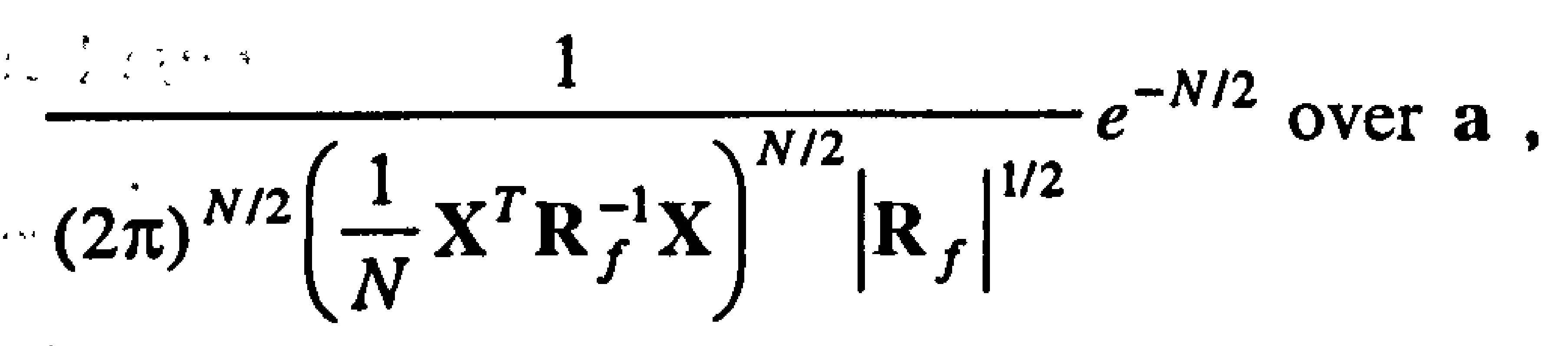

or equivalently maximize:

$$
\frac{\left|\mathbf{R}_{f}^{-1}\right|^{1 / N}}{\frac{1}{N} \mathbf{X}^{T} \mathbf{R}_{f}^{-1} \mathbf{X}}
$$

or by taking the logarithm:

$$
l=-N \ln \frac{1}{N} \mathbf{X}^{T} \mathbf{R}_{f}^{-1} \mathbf{X}+\ln \left|\mathbf{R}_{f}^{-1}\right| .
$$

We want to maximize $l$ recursively. So we need to find recursive relationships for $\left|\mathbf{R}_{f}^{-1}\right|$ and $\mathbf{X}^{T} \mathbf{R}_{f}^{-1} \mathrm{X}$ in equation (C.6).

To do so, we assume that $\mathbf{X}_{t}$ is an $\operatorname{AR}(n-1)$ process and that $l$ is maximized with respect to $\mathbf{a}_{n-1}=\left[a^{n-1}(1) \ldots a^{n-1}(n-1)\right]^{T}$ where $a^{j}(i)$ is the $i$ th AR parameter for the jh-order model. Then, $\mathbf{a}_{n-1}$ is fixed from the previous maximization and we can express $\mathbf{a}_{n}$ in terms of $\mathbf{a}_{n-1}$ by the Levinson recursion (see Appendix B equation B.1): 


$$
a^{n}(i)=\left\{\begin{array}{c}
a^{n-1}(i)+k_{n} a^{n-1}(n-i) \quad i=1 . . . n-1 \\
k_{n} \quad i=n
\end{array}\right.
$$

Thus, equation (C.6) becomes

$$
l=l_{n}=-N \ln \frac{1}{N} \mathbf{X}^{T} \mathbf{R}_{f n}^{-1} \mathbf{X}+\ln \left|\mathbf{R}_{f n}^{-1}\right|
$$

and $l_{n}$ depends only on $k_{n}$ and is maximized with respect to it.

To determine $\left|\mathbf{R}_{f n}^{-1}\right|$, we use the Toeplitz triangular decomposition (Marple 1987) since $\mathbf{R}$ is a toeplitz matrix:

$$
\mathbf{B}^{T} \mathbf{R B}=\mathbf{P}
$$

$$
\begin{aligned}
& \text { where } \mathbf{B}=\left[\begin{array}{ccccccc}
1 & a^{1}(1) & a^{2}(2) \ldots & a^{n}(n) & 0 & 0 \ldots & 0 \\
0 & 1 & a^{1}(1) \ldots & a^{n-1}(n-1) & a^{n}(n) & 0 \ldots & 0 \\
& & \ddots & & & & \\
0 & 0 & \ldots & & & & 1
\end{array}\right] N \times N \text {, } \\
& \mathbf{P}=\operatorname{diag}\left(P_{0}, P_{1}, \ldots, P_{n-1}, P_{n}, \ldots, P_{n}\right) \quad N \times N,
\end{aligned}
$$

and $P_{i}$ is the prediction error power (or white noise variance) for the $i$ th-order predictor and $P_{n}=\sigma^{2}$.

A recursion exist also for $P_{i}$ (this comes from the Levinson recursion):

$$
P_{i}=P_{i-1}\left(1-k_{i}^{2}\right) \Rightarrow P_{i}=R(0) \prod_{j=1}^{i}\left(1-k_{j}^{2}\right) \text {. }
$$

Since $|B|=1$, from (C.6), we obtain:

$$
|\mathbf{R}|=|\mathbf{P}|=\prod_{i=0}^{n-1} P_{i} \prod_{i=n}^{N-1} P_{n}
$$

and:

$$
\left|\mathbf{R}_{f n}\right|^{-1}=\left|\sigma^{2} \mathbf{R}^{-1}\right|=\sigma^{2 N} /|\mathbf{R}|=\frac{1}{\prod_{i=0}^{n-1} P_{i} / P_{n} \prod_{i=n}^{N-1} P_{n} / P_{n}}=\prod_{i=0}^{n-1} P_{n} / P_{i}
$$

Thus,

$$
\begin{gathered}
\left|\mathbf{R}_{f n}^{-1}\right|=\prod_{i=1}^{n}\left(1-k_{i}^{2}\right)^{i}=\left(1-k_{n}^{2}\right)^{n} \prod_{i=1}^{n-1}\left(1-k_{i}^{2}\right)^{i} \\
\Rightarrow\left|\mathbf{R}_{f n}^{-1}\right|=\left(1-k_{n}^{2}\right)^{n}\left|\mathbf{R}_{f n-1}^{-1}\right|
\end{gathered}
$$

that is the first desired recursive relationship. 
Now, we need to determine a recursive relationship for $\mathbf{X}^{T} \mathbf{R}_{f}^{-1} \mathbf{X}=\xi_{n}$.

To do so, note that

$$
\xi_{n}=\mathbf{X}^{T} \mathbf{R}_{f}^{-1} \mathbf{X}=\mathbf{a}_{n}^{\prime T} \mathbf{S}_{n} \mathbf{a}_{n}^{\prime}
$$

where $\mathbf{a}_{n}^{\prime}=\left[1 a^{n}(1) \ldots a^{n}(n)\right]^{T}$ and

$$
\mathbf{S}_{n}=\left[S_{i j}=\sum_{t=1}^{N-i-j} X(t+i) X(t+j)\right]_{0 \leq i, j \leq n} \text { is a symmetric matrix. }
$$

Using the recursion (C.7), we partition (C.12) into

$$
\xi_{n}=\left[\begin{array}{c}
1 \\
\left(\mathbf{I}_{n-1}+k_{n} \mathbf{J}_{n-1}\right) \mathbf{a}_{n-1} \\
k_{n}
\end{array}\right]^{T} \cdot S_{n} \cdot\left[\begin{array}{c}
1 \\
\left(\mathbf{I}_{n-1}+k_{n} \mathbf{J}_{n-1}\right) \mathbf{a}_{n-1} \\
k_{n}
\end{array}\right]
$$

where $\mathbf{I}_{n-1}$ is the identity matrix of dimension $(n-1) \times(n-1)$,

$\mathbf{J}_{n-1}=\left[\begin{array}{ccc}0 & \cdots & 1 \\ & \therefore & \\ 1 & \cdots & 0\end{array}\right]$ is the reflection matrix of dimension $(n-1) \times(n-1)$ and

$$
\mathbf{S}_{n}=\left[\begin{array}{ccc}
S_{00} & \mathbf{q}_{n-1}^{T} & S_{0 n} \\
1 \times 1 & 1 \times(n-1) & 1 \times 1 \\
\hline \mathbf{q}_{n-1} & \mathbf{S}_{n-2} & \mathbf{p}_{n-1} \\
(n-1) \times 1 & (n-1) \times(n-1) & (n-1) \times 1 \\
\hline S_{n 0} & \mathbf{p}_{n-1}^{T} & S_{n n} \\
1 \times 1 & 1 \times(n-1) & 1 \times 1
\end{array}\right] .
$$

Since $\mathbf{a}_{n}^{\prime}$ can be further decomposed as $\mathbf{a}_{n}^{\prime}=\left[\begin{array}{c}\mathbf{a}_{n-1}^{\prime} \\ 0\end{array}\right]+k_{n}\left[\begin{array}{c}0 \\ \mathbf{b}_{n-1}^{\prime}\end{array}\right]$ with $\mathbf{b}_{n-1}^{\prime}=\mathbf{J}_{n} \mathbf{a}_{n-1}^{\prime}$, we finally obtain

$$
\xi_{n}=\xi_{n-1}+2 c_{n} k_{n}+d_{n} k_{n}^{2}
$$

where $c_{n}=\mathbf{a}_{n-1}^{\prime T}\left[\begin{array}{cc}\mathbf{q}_{n-1}^{T} & S_{0 n} \\ \mathbf{S}_{n-2} & \mathbf{p}_{n-1}\end{array}\right] \mathbf{b}_{n-1}^{\prime}, \quad d_{n}=\mathbf{b}_{n-1}^{\prime T}\left[\begin{array}{cc}\mathbf{S}_{n-2} & \mathbf{p}_{n-1} \\ \mathbf{p}_{n-1}^{T} & S_{n n}\end{array}\right] \mathbf{b}_{n-1}^{\prime}$ and

$\mathbf{b}_{n-1}^{\prime}=\left[a^{n-1}(n-1) \ldots a^{n}(1) 1\right]^{T}$.

Thus,

$$
l_{n}=-N \ln \frac{1}{N}\left(\xi_{n-1}+2 c_{n} k_{n}+d_{n} k_{n}^{2}\right)+\ln \left|\mathbf{R}_{f n-1}^{-1}\right|+n \ln \left(1-k_{n}^{2}\right) .
$$


It is assumed that $k_{1} \ldots k_{n-1}$ have been estimated. Then, the terms $\xi_{n-1}, c_{n}, d_{n}$ and $\left|\mathbf{R}_{f n-1}^{-1}\right|$ can be computed and treated as constants. Therefore, to maximize $l_{n}$ with respect to $k_{n}$, we set $\frac{\partial l_{n}}{\partial k_{n}}=0$ and finally obtain

$$
k_{n}^{3}+\frac{(N-2 n) c_{n}}{(N-n) d_{n}} k_{n}^{2}-\frac{n \xi_{n-1}+N d_{n}}{(N-n) d_{n}} k_{n}-\frac{N c_{n}}{(N-n) d_{n}}=0 .
$$

The equation provides either three real roots or one real root and two complex conjugate roots. This depends on $d_{n}\left(d_{n}>0, d_{n}=0\right.$ and $\left.d_{n}<0\right)$. Kay (1983) proved that at least one real root within the interval $[-1,1]$ exists

Once the estimate of $k_{n}$ is determined by this cubic equation, from equation (C.5) $\sigma^{2}$ can be obtained

$$
\sigma^{2}=\frac{1}{N} \xi_{n}=\frac{1}{N}\left(\xi_{n-1}+2 c_{n} k_{n}+d_{n} k_{n}^{2}\right)
$$

To ease the computational burden, determining $c_{n}$ and $d_{n}$, we can use:

$$
\left\{\begin{array}{c}
{\left[\mathbf{S}_{n}\right]_{i j}=\left[\mathbf{S}_{n-1}\right]_{i j} \quad 0 \leq i, j \leq n-1} \\
{\left[\mathbf{S}_{n}\right]_{i j}=\left[\mathbf{S}_{n-1}\right]_{i-1, j-1}-X(i) X(j)-X(N+1-i) X(N+1-j) \quad i=n, j=1 \ldots n .} \\
{\left[\mathbf{S}_{n}\right]_{i j}=\left[\mathbf{S}_{n}\right]_{j i} \quad i=1 \ldots n, j=n}
\end{array}\right.
$$

Note that if $\mathbf{X}_{t}$ is an $\mathrm{AR}(1)$ process, then the solution of (C.16) produces the exact MLE.

Once this reflection coefficient is obtained, the AR PSD estimate can be computed as for the Burg method described in the previous appendix (see equation B.7).

Using real data, this method provides symmetric spectral estimates. Since it was difficult to solve the cubic equation (C.16) in the complex case ( $k_{n}$ appears as its modulus and as itself), we left this method aside. 